\title{
The gut in control of health and disease
}

\author{
Citation for published version (APA):
}

van der Beek, C. M. (2017). The gut in control of health and disease: unraveling the role of short-chain fatty acids in human metabolism. [Doctoral Thesis, Maastricht University]. Datawyse / Universitaire Pers Maastricht. https://doi.org/10.26481/dis.20171004cvdb

\section{Document status and date:}

Published: 01/01/2017

DOI:

10.26481/dis.20171004cvdb

Document Version:

Publisher's PDF, also known as Version of record

\section{Please check the document version of this publication:}

- A submitted manuscript is the version of the article upon submission and before peer-review. There can be important differences between the submitted version and the official published version of record.

People interested in the research are advised to contact the author for the final version of the publication, or visit the DOI to the publisher's website.

- The final author version and the galley proof are versions of the publication after peer review.

- The final published version features the final layout of the paper including the volume, issue and page numbers.

Link to publication

\footnotetext{
General rights rights.

- You may freely distribute the URL identifying the publication in the public portal. please follow below link for the End User Agreement:

www.umlib.nl/taverne-license

Take down policy

If you believe that this document breaches copyright please contact us at:

repository@maastrichtuniversity.nl

providing details and we will investigate your claim.
}

Copyright and moral rights for the publications made accessible in the public portal are retained by the authors and/or other copyright owners and it is a condition of accessing publications that users recognise and abide by the legal requirements associated with these

- Users may download and print one copy of any publication from the public portal for the purpose of private study or research.

- You may not further distribute the material or use it for any profit-making activity or commercial gain

If the publication is distributed under the terms of Article $25 \mathrm{fa}$ of the Dutch Copyright Act, indicated by the "Taverne" license above, 


\section{THE GUT IN CONTROL OF HEALTH AND DISEASE UNRAVELING THE ROLE OF SHORT-CHAIN FATTY ACIDS IN HUMAN METABOLISM}




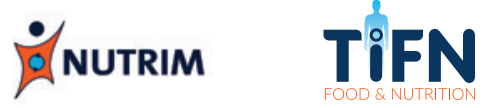

The work presented in this dissertation was performed within NUTRIM School of Nutrition and Translational Research in Metabolism, accredited by the Royal Netherlands Academy of Arts and Sciences, and within the framework of TI Food and Nutrition.

Cover design and layout: Evelien Jagtman, evelienjagtman.com

Printed by: Datawyse, Universitaire Pers Maastricht

\section{ISBN: 978-94-6295-680-3}

Copyright (c) 2017 Kirsten van der Beek

All rights reserved. No part of this dissertation may be reproduced, distributed, or transmitted in any form or by any means, including photocopying, recording, or other electronic or mechanical methods, without the prior written permission of the author or publisher, except in the case of brief quotations embodied in critical reviews and certain other noncommercial uses permitted by copyright law.

Printing of this thesis was financially supported by Maastricht University, TI Food and Nutrition, Nederlandse Vereniging voor Gastroenterologie, Tramedico BV, Pentax Medical, Dr. Falk Pharma and Medische Laboratoria Dr. Stein \& Collegae. 


\title{
THE GUT IN CONTROL OF HEALTH AND DISEASE UNRAVELING THE ROLE OF SHORT-CHAIN FATTY ACIDS IN HUMAN METABOLISM
}

\author{
PROEFSCHRIFT \\ Ter verkrijging van de graad van doctor aan de Universiteit Maastricht, \\ op gezag van de Rector Magnificus, prof. dr. Rianne M. Letschert \\ volgens het besluit van het College van Decanen, \\ in het openbaar te verdedigen \\ op woensdag 4 oktober 2017 om 12.00 uur
}

door

Christina Maria van der Beek 


\section{Promotores}

Prof. dr. C.H.C. Dejong

Prof. dr. A.A.M. Masclee

\section{Copromotor}

Dr. K. Lenaerts

\section{Beoordelingscommissie}

Prof. dr. N.D. Bouvy (voorzitter)

Dr. D.M.A.E. Jonkers

Prof. dr. M. Nieuwdorp, Academisch Medisch Centrum Amsterdam

Prof. dr. J. Plat

Prof. dr. K. Verbeke, Katholieke Universiteit Leuven, België 


\section{TABLE OF CONTENTS}

Chapter 1 General Introduction 7

Chapter 2 Distal, not proximal, colonic acetate infusions promote fat 31 oxidation and improve metabolic markers in overweight/ obese men

Chapter 3 The prebiotic inulin improves substrate metabolism and promotes short-chain fatty acid production in overweight to obese men

Chapter $4 \quad$ Supplementation of diet with galacto-oligosaccharides increases bifidobacteria, but not insulin sensitivity, in obese prediabetic individuals

Chapter $5 \quad$ Expression of short-chain fatty acid receptors GPR41, GPR43, 109 and GPR109a is higher in the human ileum and proximal colon compared with the distal colon

Chapter $6 \quad$ Hepatic uptake of rectally administered butyrate prevents an $\quad 125$ increase in systemic butyrate concentrations in humans

Chapter 7 Role of short-chain fatty acids in colonic inflammation, carcinogenesis, and mucosal protection and healing

Chapter $8 \quad$ General Discussion

$\begin{array}{ll}\text { Addendum } & \text { Summary } \\ & \text { Samenvatting } \\ & \text { Valorization } \\ & \text { Curriculum vitae } \\ & \text { List of publications } \\ & \text { Dankwoord }\end{array}$




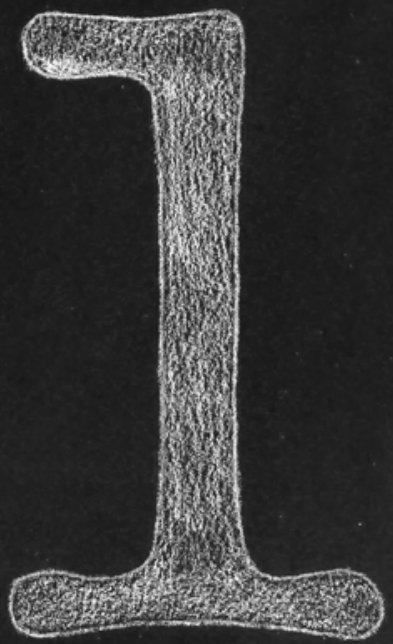




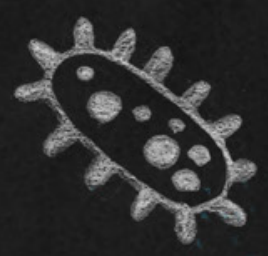

\section{CHAPTER 1}

General introduction 
"All disease begins in the gut" - Hippocrates (Kos, ca. 460 v.Chr. - Larissa, 370 v.Chr.) Already more than two thousand years ago, Hippocrates, the father of modern medicine, understood the importance of the gut in maintaining human health. Nowadays, science is pointing towards the gut not only as origin of several diseases, but also as site of treatment of these diseases. Surprisingly, these do not only involve gut diseases, but also include metabolic disorders such as obesity and type 2 diabetes. 


\section{THE GASTROINTESTINAL TRACT}

The human gastrointestinal tract has a surface area of approximately $300-400 \mathrm{~m}^{2}$ and is thereby the largest organ in the body. ${ }^{1}$ When food enters the intestinal tract through the mouth, several processes are set in motion. ${ }^{2}$ Digestion is a complicated process that already starts in the mouth by chewing of the food and breakdown of starch. The tongue pushes the food into the esophagus towards the stomach, where it is mixed with acidic gastric juice, which contains several digestive enzymes. The stomach passes the food mash in small portions into the small intestine. The small intestine has three distinct regions - the duodenum, jejunum, and ileum. In the duodenum, the first part of the small intestine, the food is exposed to bile and pancreatic juice, released from the liver via the biliary system and pancreas respectively. The secretion of hormones and subsequently digestive enzymes ensures digestion of food into nutrients. The three major classes of nutrients that undergo digestion are proteins, lipids (fats), and carbohydrates. Most of these nutrients are digested and absorbed in the small intestine. The wall of the small intestine is folded and has small extensions, the so-called villi and microvilli. These increase the surface area of the small intestine, and thus the absorption property, to an average of $250 \mathrm{~m}^{2}$. The terminal ileum is the final section of the small intestine and joins to the first part of the large intestine, the cecum.

The large intestine, i.e. the colon, receives indigestible food substances and absorbs mainly water, salts, and vitamins from the remaining waste. Food waste enters the first part of the colon, the cecum, and then passes through the ascending colon, transverse colon, and descending colon into the sigmoid colon and the rectum (Figure 1.1). In the rectum, the food waste is stored until defecation. Next to its function in digestion and absorption, the human gut hosts over 700 species of bacteria, with a total of $>10^{14}$ unique bacteria. ${ }^{3,4}$ Most of these bacteria are located in the colon. All bacteria residing in the gut are collectively called the microbiota. The microbial genome encodes for $>10.000 .000$ genes, and hence, the number of genes in the gut microbiome exceeds that of the human genome by 150 times. 5,6 As such, the gut microbiota complements our own genome by performing essential functions such as metabolism of nutrients, drugs, and bile acids, vitamin production, maintenance of epithelial integrity,

Figure 1.1.

Designation of colonic segments

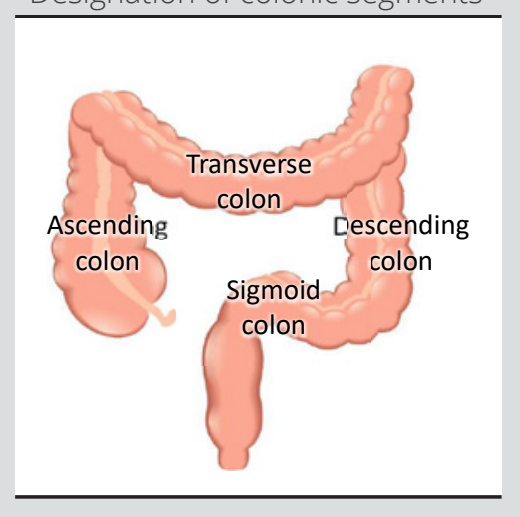


and supporting immunity. ${ }^{7-10}$ Importantly, the gut microbiota is rapidly gaining worldwide interest due to the increasing awareness that intestinal microbiota composition and function is closely associated with health, and thus with the development and progression of a variety of disorders. ${ }^{11-14}$

\section{HUMAN MICROBIOTA AND SHORT-CHAIN FATTY ACIDS}

Infants are born germ-free, i.e. without gut microbiota, but rapidly after birth the infant's gut is colonized by bacteria coming from the mother and the surrounding environment. ${ }^{15}$ The adult gut microbiota is mainly dominated by three phyla, Bacteroidetes, Firmicutes, and Actinobacteria, combined comprising more than $90 \%$ of all bacteria. ${ }^{16}$ The mature gut microbiota is relatively stable and every individual has a unique microbial composition and diversity. ${ }^{17}$

The gut microbiome is particularly enriched in genes encoding enzymes essential for the metabolism of several macronutrients. ${ }^{18}$ As such, the human gut microbiota has the ability to ferment otherwise indigestible food components into short-chain fatty acids (SCFAs), mostly acetate, propionate, and butyrate, and the gasses $\mathrm{CO}_{2}$ and $\mathrm{H}_{2}$. SCFAs are produced mainly through saccharolytic fermentation of carbohydrates that escape digestion and absorption in the small intestine. Of the three main SCFAs, butyrate is considered most important for maintenance of colonic health. In physiological circumstances only a small amount of butyrate is found in the systemic circulation. ${ }^{19,20}$ On the contrary, acetate and propionate are largely taken up by the colon and released into the systemic circulation to affect energy regulation, lipid and glucose metabolism, and inflammation in various tissues. ${ }^{21,22}$

\section{Colonic short-chain fatty acid production, uptake, and systemic appearance}

The rate and amount of SCFA production is determined by several factors, including substrate source and diet, microbial abundance and composition, and gut transit time. ${ }^{7,21,23}$ Dietary fibers, i.e. prebiotics, are the most potent substrates for generating SCFAs and consumption of a high fiber diet increases colonic SCFA concentrations considerably. ${ }^{23-25}$ Moreover, several bacteria are considered beneficial due to their capacity to produce SCFAs, e.g. the genus Bifidobacterium for its acetate-producing capacity and well-known butyrate-producing species such as Faecalibacterium prausnitzii, Roseburia intestinalis, and Lactobacillus plantarum. ${ }^{26,27}$ An accelerated transit through the colon diminishes substrate availability for microbial fermentation, which might lead to reduced SCFA production. ${ }^{21}$ 
Under normal conditions, SCFA production occurs predominantly in the cecum and proximal part of the colon, where most substrates are available for bacterial fermentation. These sources are increasingly depleted along the colon towards the distal part, thereby decreasing SCFA production. ${ }^{28}$ Depending on the diet, the total concentration of SCFAS decreases from $70-140 \mathrm{mM}$ in the proximal colon to $20-70 \mathrm{mM}$ in the distal colon. ${ }^{24}$ The SCFA ratio throughout the colon is 3:1:1 for acetate, butyrate, and propionate respectively. This ratio is similar for the proximal and distal colon, reflecting an equal uptake of all three SCFAs along the large bowel. ${ }^{28}$ The majority of SCFAs are taken up via several active transport mechanisms, but passive diffusion also plays a role. ${ }^{29-32}$ The fraction of total SCFAs that is not metabolized by colonocytes is transported over the basolateral membrane into the portal vein to reach the liver. ${ }^{33,34}$ The liver metabolizes all butyrate and a large amount of propionate released from the gut, whilst acetate is not taken up by the liver in large amounts. ${ }^{20}$ Therefore acetate is the only SCFA that reaches the circulation in considerable amounts. It is conceivable that rectally produced SCFAs partly bypass the liver as the venous drainage of this colonic segment is partly via the internal iliac vein directly into the systemic circulation. The uptake of SCFAs by the colon is a rapid and efficient concentration-dependent process. Only 5 - 10\% of the total amount of SCFAs that are present in the proximal large bowel appear in the distal part of the colon and are excreted with the feces. ${ }^{24}$

\section{Colonic short-chain fatty acid receptor activation}

In addition to uptake and metabolism by colonocytes, SCFAs can bind to specific G-protein coupled receptors (GPRs) to act as signaling molecules: GPR41 (also named free fatty acid receptor 3 (FFAR3)), GPR43 (or free fatty acid receptor 2 (FFAR2)), and GPR109a. ${ }^{35-37}$ All three receptors are located throughout the body, and are highly expressed in adipose tissue, immune cells, and intestinal cells. ${ }^{35-39}$ Colonic SCFA receptors play a role in local processes such as enhancing the intestinal barrier, lowering intestinal inflammation, and inhibiting carcinogenesis. ${ }^{19}$ In addition, GPR41 and GPR43 are expressed by L-cells, which are specialized hormone-releasing cells in the terminal ileum and colon. Upon activation by these receptors, L-cells secrete the gut hormones peptide YY (PYY) and glucagon-like peptide-1 (GLP-1) into the systemic circulation, which thereby might affect human metabolism. ${ }^{40-42}$ 


\section{SHORT-CHAIN FATTY ACIDS IN METABOLISM}

\section{Energy regulation}

Overweight, obesity, and related disorders such as type 2 diabetes represent a rapidly growing health threat. ${ }^{43}$ Overweight is defined as having a body mass index (BMI) $>25 \mathrm{~kg} /$ $\mathrm{m}^{2}$ and obesity as a BMI $>30 \mathrm{~kg} / \mathrm{m}^{2}$. In 2014, more than 1.9 billion adults worldwide were overweight, and over 600 million were obese. ${ }^{44}$ The economic contribution of obesity is in the range of 2 - 7\% of total health care costs. ${ }^{45}$ As such, obesity is placing a tremendous economic burden on society. The etiology of obesity is attributed to a positive energy balance with increased energy harvesting and intake, and reduced energy expenditure. ${ }^{46}$ Growing evidence supports that the gut microbiota and SCFAs play an important role in energy regulation by affecting both sides of this energy balance. ${ }^{47,48}$

The finding that the gut microbiota plays a role in energy regulation has first been described in animal studies. It was shown that germ-free mice had a considerably lower body weight and body fat percentage compared with conventionally raised mice which were colonized with microbiota. ${ }^{47}$ Moreover, germ-free mice were resistant to diet-induced obesity when fed a high-fat diet. ${ }^{53,54}$ Of interest, colonization of the germ-free mice with microbiota from conventionally raised mice resulted in an increase in body weight. ${ }^{47}$ The protection of germ-free mice against obesity has been attributed to a reduced capacity to harvest energy from the diet in the form of SCFAs. As a matter of fact, fermentation of polysaccharides into SCFAs in the human colon has been suggested to contribute up to $10 \%$ of daily energy requirements. ${ }^{49}$ However, human data are scarce up to now. Since it is impossible for humans to have a gut that is not colonized with microbiota, the relevance of this energy harvest from dietary fibers is unknown.

In contrast to the conception that SCFAs lead to an increase in body weight, dietary administration of SCFAs protected mice from diet-induced obesity and prevented body weight gain in obese human subjects. ${ }^{50-52}$ SCFAs contribute to a decreased energy intake and satiety by increasing the release of the satiety hormones PYY and GLP-1.53-55 PYY and GLP-1 suppress appetite, ${ }^{56}$ and in addition, have been shown to reduce gastrointestinal transit and contribute to the ileal brake thereby inhibiting gastric emptying. ${ }^{57,58}$ Moreover, SCFAs might stimulate secretion of the satiety hormone leptin by adipocytes and might have direct effects on the hypothalamus, thereby inhibiting hunger. ${ }^{59-61} \mathrm{Sev}$ eral human studies have investigated the effect of ingesting dietary fibers and SCFAs on body weight control and satiety, showing lower subjective scores on hunger and satiety, reduced energy intake and considerable weight loss after dietary fiber intake which was associated with an increase in plasma PYY and GLP-1.62,63

In addition, SCFAs have been shown to increase energy expenditure. The gut microbiota has been found to be involved in the expression of $5^{\prime}$ adenosine monophosphate-acti- 
vated protein kinase (AMPK), an enzyme that plays a role in cellular energy homeostasis. ${ }^{64}$ In line, rodent-derived data indicate that SCFAs increase energy expenditure by activating AMPK activity in liver and skeletal muscle. ${ }^{51,52,65,66}$ Whether these effects can be translated into the human condition is still unknown.

In conclusion, SCFAs have been suggested to decrease energy intake, and increase both satiety and energy expenditure, thereby influencing body weight regulation. However, the interactions between gut microbiota, SCFAs, and host energy metabolism are complex and remain to be elucidated in humans. The suggested mechanisms by which SCFAs influence the host's metabolism are summarized in Figure 1.2.

Figure 1.2. Suggested mechanisms by which SCFAs influence the host's metabolism
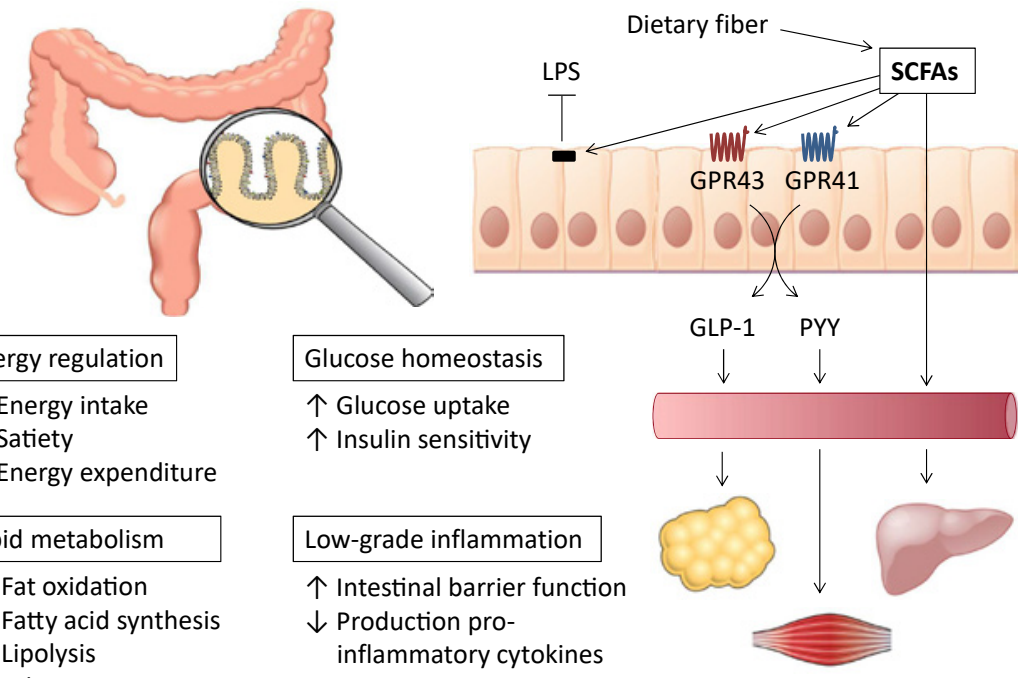

\begin{tabular}{l}
\hline Energy regulation \\
$\downarrow$ Energy intake \\
$\uparrow$ Satiety \\
$\uparrow$ Energy expenditur
\end{tabular}

\begin{tabular}{l}
\hline Lipid metabolism \\
$\uparrow$ Fat oxidation \\
$\downarrow$ Fatty acid synthesis \\
$\downarrow$ Lipolysis \\
$\uparrow$ Adipogenesis
\end{tabular}

$\uparrow$ FFA uptake in adipose tissue

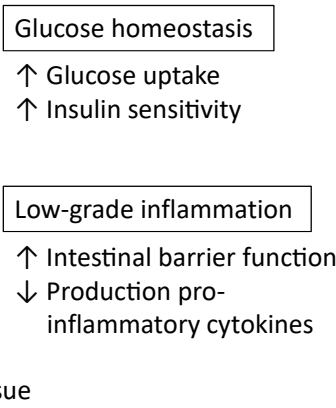

Glucose homeostasi

$\uparrow$ Glucose uptake

个 Insulin sensitivity

Abbreviations: FFA, Free fatty acid; LPS, Lipopolysaccharide; GLP-1, glucagon-like peptide-1; GPR, G-protein coupled receptor; PYY, Peptide YY; SCFAs, Short-chain fatty acids.

\section{Lipid metabolism}

Disturbances in lipid metabolism are involved in the etiology of obesity and obesity-related disorders. In healthy conditions, most of the dietary lipids are stored in adipose tissue as triacylglycerol (TAG). ${ }^{67}$ In order to provide fatty acids for oxidation, intracellular breakdown of lipids, i.e. lipolysis, takes place, during which TAG is degraded into free fatty acids 
(FFAs) and glycerol, which are released into the circulation. Moreover, extracellular lipolysis whereby FFAs are freed from plasma lipids by lipoprotein lipase (LPL), adds to the total lipid balance in plasma and metabolic organs. These processes are under strict regulation by hormones such as insulin and ANGPTL4. ${ }^{68,69}$ In addition, insulin induces FFA uptake from the circulation into adipocytes by activating $L P L$, contributing to a lowering in plasma lipids. In obesity, adipose tissue malfunction occurs due to an increase in adipose tissue mass. Adipocytes become enlarged (i.e. undergo hypertrophy) and the storage capacity of adipocytes is exceeded. This results in systemic lipid overflow, an increase in plasma triglycerides and FFAs, and ectopic fat deposition in organs such as the liver, skeletal muscle, and pancreas if lipid supply to these organs exceeds their fat oxidative capacity. ${ }^{70,71}$

Already in 1968, the anti-lipolytic effects of acetate were discovered by Crouse et al. ${ }^{72}$, who showed a decrease in plasma FFAs after oral administration of acetate in humans. Later on, several human studies have investigated lipid metabolism after SCFA suppletion and showed a decrease in adipocyte lipolysis after gastric and rectal SCFA infusions. ${ }^{73-78}$ More specifically, SCFAs have been found to regulate the balance between fatty acid synthesis, oxidation, and breakdown in vitro. Fatty acid oxidation in liver and muscle tissue is activated by SCFAs, ${ }^{51}$ while hepatic fatty acid synthesis and adipose tissue lipolysis are inhibited. 52,79,80 Moreover, in vitro and rodent models suggest that SCFAs might be involved in adipogenesis, the process of adipocyte differentiation, and increase LPL-mediated FFA uptake into the adipocytes.81-84 The combination of a decrease in lipolysis, increase in adipogenesis, and increase in FFA uptake into the adipocytes might lead to a higher lipid storage capacity in white adipose tissue and a lower FFA supply to liver and muscle tissue, resulting in lower ectopic fat storage. It has been suggested that SCFAs increase oxidative metabolism in liver and muscle by activating AMPK. ${ }^{51,52,66,85,86}$ Indeed, an animal study by den Besten et al. ${ }^{66}$ showed an increase in the expression of mitochondrial uncoupling protein 2 and elevations of the AMP/ATP ratio. This led to a switch from lipid synthesis to fat oxidation via an AMPK-dependent mechanism in liver and muscle. Of importance, SCFAs can directly be converted into acetyl-CoA, which enters the citric acid cycle and can be used for oxidation. ${ }^{87}$

In conclusion, the prevention of dietary-induced obesity by SCFAs might be attributed to an increase in fatty acid oxidation in liver and adipose tissue and an increase in fat storage in white adipose tissue. This would result in a decrease in plasma FFAs and decrease in ectopic lipid accumulation after SCFA application. Further well controlled studies in humans are needed to dissect the exact role of SCFAs in lipid homeostasis. 


\section{Glucose homeostasis and insulin sensitivity}

Obesity increases the risk of multifactorial diseases such as type 2 diabetes. As already described, obese subjects develop impairment in adipose tissue lipid buffering capacity, resulting in lipid overflow and ectopic fat deposition in liver and skeletal muscle. Ectopic fat deposition is associated with insulin resistance, thereby disrupting glucose uptake and glycogen storage. ${ }^{88}$ The role of the gut microbiota in glucose homeostasis was recently shown by a study where fecal microbiota transplantation from lean donors to insulinresistant patients with metabolic syndrome caused an improvement in insulin sensitivity and increase in levels of butyrate-producing intestinal microbiota. ${ }^{89}$ Interestingly, another study suggests that the gut microbiome acts as a novel biomarker for the prediction of type 2 diabetes. ${ }^{90}$ Altogether, there is accumulating evidence that suggests that the microbiota directly modulates glucose homeostasis and insulin sensitivity, possibly via SCFA production.

As already discussed, SCFAs affect the secretion of the hormones PYY and GLP-1 via GPR activation. PYY is known as a satiety hormone but it might also increase glucose

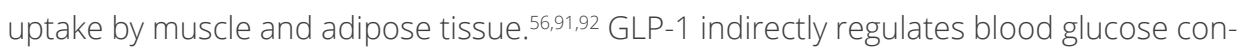
centration by increasing the secretion of insulin and decreasing the secretion of glucagon by the pancreas. ${ }^{93}$ Intracolonic infusions of SCFAs and intake of fibers both increased plasma GLP-1 concentrations and glucose uptake by adipose tissue. ${ }^{54,55,94}$ In addition, AMPK activation by SCFAs might cause a decrease in hepatic gluconeogenesis. ${ }^{95}$ Moreover, propionate adds to glucose homeostasis by acting as a precursor for hepatic gluconeogenesis, however an elegant isotope study revealed that only $6 \%$ of colonic propionate is used to form glucose in humans. ${ }^{22}$

Human studies investigating the direct effects of SCFAs on glucose homeostasis and insulin sensitivity are scarce. A study in which acetate was administered both rectally and intravenously did not show any direct effect on blood glucose and insulin concentrations in hyperinsulinemic women, although an effect on plasma PYY was found. ${ }^{.5}$ In line, other human studies found no beneficial effect of rectal acetate administration on plasma glucose and insulin concentrations. ${ }^{74,75}$ Moreover, rectal propionate was even indicated to increase plasma glucose by $\sim 8 \%{ }^{74,75}$ On the other hand, oral propionate ingestion during seven weeks caused a decrease in fasting glucose concentrations in healthy subjects, ${ }^{96}$ and oral propionate ingestion for one week decreased $2 \mathrm{~h}$ postprandial glucose concentrations. ${ }^{97}$

In summary, although animal studies suggest that SCFAs appear to beneficially affect glucose metabolism by normalizing plasma glucose concentrations and increasing glucose handling, human data are scarce and inconclusive. To what extent these effects occur directly via a hepatic AMPK regulation pathway or indirectly via the gut derived hormones PYY and GLP-1 remains to be established. 


\section{Low-grade inflammation}

Obesity and insulin resistance have been linked to systemic low-grade inflammation. ${ }^{98,99}$ When adipose tissue expands, inflammation develops and adipocytes increase production of pro-inflammatory cytokines, such as TNF-a and interleukins (IL). As a result, these cytokines inhibit the actions of insulin on the liver, muscle, and adipose tissue. ${ }^{100}$ Moreover, pro-inflammatory cytokines may cause an impaired gut barrier function in obese subjects, ${ }^{101}$ increasing the chance of translocation of bacteria and/or their cell wall components, i.e. endotoxins such as lipopolysaccharides (LPS), into the systemic circulation. ${ }^{102}$ Systemic LPS leads to activation of the immune system, thereby stimulating cytokine release from adipocytes, and has thus been associated with obesity and insulin resistance. ${ }^{103-105}$ Furthermore, LPS translocation might induce an inflammatory cascade in the liver, ultimately leading to development of steatohepatitis and hepatic insulin resistance. ${ }^{106}$

Several in vitro studies have shown that SCFAs suppress the immune response by reducing the production of pro-inflammatory cytokines IL-6 and IL-12 and increasing the production of the anti-inflammatory cytokine IL-10 in human immune cells. ${ }^{107-110}$ Incubating human adipose tissue with propionate resulted in a reduced secretion of the pro-inflammatory cytokines IL-4, IL-10, and TNF-a. ${ }^{111}$ It is possible that suppression of transcription factor NF-kB by SCFAs is responsible for this immune modulating effect. ${ }^{112}$ Moreover, SCFAs have also been shown to induce differentiation of regulatory $T$-cells ( $T_{\text {reg }}$ cells). ${ }^{113,114}$ $T_{\text {reg }}$ cells play an important role in limiting inflammatory responses via production of antiinflammatory cytokines, and their numbers are decreased in adipose tissue from obese patients. ${ }^{113,115}$

In vitro ${ }^{116}$ and ex vivo ${ }^{117}$ studies have pointed towards an improved colonic barrier function after SCFA supplementation. Ferreira et al. ${ }^{118}$ have shown improved intestinal barrier in a mouse model with colitis after supplementing drinking water with a mixture of SCFAs or butyrate alone. In healthy volunteers, improved intestinal barrier function has been observed after chronic intake of inulin, a prebiotic that is known to increase colonic SCFA concentrations. ${ }^{119}$ Furthermore, SCFAs might directly decrease LPS-induced production of inflammatory cytokines in vitro. 108,112,120 Human studies investigating the role of SCFAs on low-grade systemic inflammation are scarce, however Freeland et $a .{ }^{55}$ showed that rectal and intravenous acetate administration decreased plasma TNF-a concentrations in obese women.

In conclusion, SCFAs have the potential to prevent chronic low-grade inflammation by strengthening the intestinal barrier and by influencing production of inflammatory cytokines. However, evidence from human studies on systemic low-grade inflammation is scarce. 


\section{SHORT-CHAIN FATTY ACIDS IN INTESTINAL HEALTH}

As already described, SCFAs contribute to an improved colonic barrier function. Moreover, SCFAs have been found to be involved in the etiology of colonic inflammatory diseases and colonic carcinogenesis. Several underlying mechanisms have been proposed to contribute to the effects of SCFAs on colonic health. In short, SCFAs act as energy supply to colonocytes, $^{121-123}$ and bind to GPRs located in the intestine and intestinal immune cells to activate downstream signaling pathways.36-38,124 In addition, they inhibit histone deacetylases (HDACs) in both colonocytes and mucosal immune cells. ${ }^{125}$ HDACs are enzymes that prevent DNA from transcription and thereby regulate gene expression in several complex processes. As a result, in vitro and ex vivo studies show that SCFAs strengthen colonic barrier function ${ }^{116,117,126}$ and increase mucus production, ${ }^{127-131}$ reduce oxidative stress, ${ }^{132-}$ 134 support the immune system by decreasing the production of pro-inflammatory cytokines ${ }^{107-110}$ and influence apoptosis, proliferation, and cell differentiation in cancerous cells. ${ }^{122,123,135-137}$ However, convincing human evidence in gastrointestinal diseases, such as inflammatory bowel disease (IBD), diversion colitis, radiation proctitis, pouchitis, and colon carcinogenesis and mucosal healing is yet lacking and other therapeutic strategies need to be investigated. SCFA enemas, especially with butyrate, are increasingly used for treatment and prevention of colonic diseases. It should be acknowledged that high systemic concentrations of butyrate may cause side effects.120,121 Therefore, studies on intestinal SCFA release and hepatic clearance of rectally applied butyrate are warranted. 


\section{PREBIOTICS}

By ingestion of dietary fibers, i.e. prebiotics, gut microbial composition and activity, including SCFA fermentation, can be influenced. A prebiotic is defined as a non-digestible food ingredient that beneficially affects the host by selectively stimulating the growth and/or activity of one or a limited number of bacterial species in the colon. ${ }^{138}$ Recently, dietary fibers have raised much attention, as they exert beneficial effects on body weight, food intake, glucose homeostasis, and insulin sensitivity. ${ }^{139}$

Two commonly used prebiotics are inulin and galacto-oligosaccharides (GOS). Inulin is a polysaccharide derived from plant extracts, mostly chicory. GOS is derived from $\beta$-galactosidase-induced conversion of lactose. Several human studies have shown that daily supplementation with these prebiotics increases fecal bifidobacteria, which have been proposed to act beneficially on human metabolism, e.g. by increasing acetate production. ${ }^{140-142}$ Moreover, in vitro models have shown that both inulin and GOS are microbially fermented into SCFAs in considerable amounts. ${ }^{143-147}$ Longer-term inulin supplementation caused reductions in body weight, BMI, as well as energy and fat intake in patients with type 2 diabetes. ${ }^{148}$ Replacing digestible carbohydrates with inulin for three to eight weeks in healthy subjects reduced plasma lipid concentrations, ${ }^{141,149-153}$ and lowered fasting glucose and insulin concentrations. ${ }^{148,152,154}$ Moreover, twelve-week supplementation of a GOS mixture improved insulin and lipid homeostasis and attenuated low-grade systemic inflammation in overweight participants, ${ }^{155}$ and improved systemic and fecal inflammatory markers in elderly subjects. ${ }^{156}$ The exact mechanism by which inulin and GOS influence the host's substrate metabolism and whether SCFAs contribute to the observed metabolic effects in humans remains to be investigated. 


\section{AIM AND OUTLINE OF THE THESIS}

As human evidence concerning the metabolic effects of SCFAs is scarce, we aimed to investigate the role of SCFAs in human metabolism in this thesis. In the first part of this thesis (Chapter 2 - 4), we describe several intervention trials investigating the role of SCFAs in substrate and energy metabolism of overweight to obese human subjects. Chapter 2 describes the results of a placebo-controlled randomized crossover study in which we investigated the acute metabolic effects of colonic SCFA administration. Also, we studied if the site of colonic SCFA administration, i.e. the distal or proximal part of the colon, differentially affected metabolism. We developed a unique technique, using colonic catheters to infuse the SCFA acetate into the distal and proximal colon of the subjects, and assessed if distal or proximal colonic infusions differentially affected fat oxidation, energy expenditure, and several metabolic and inflammatory parameters. In order to investigate a more physiological situation, we studied the acute metabolic effects of the prebiotic inulin in Chapter 3. In this placebo-controlled trial, we investigated if a single oral ingestion of inulin affected substrate metabolism, circulating metabolites and hormones, SCFA concentrations, and appetite in obese subjects. The application of a stable isotope technique allowed us to assess whether inulin was fermented into SCFAs and to explore the kinetics of SCFA production and availability in humans. In Chapter 4 the acetate promoting prebiotic GOS was selected to investigate if outcomes of the studies described in Chapter 2 and 3 could be translated into an improved metabolic profile after longer-term intake. In this randomized, placebo-controlled intervention study, we investigated the effects of twelve-week GOS supplementation on both microbiota composition and functionality, i.e SCFA production, and combined this with detailed host metabolic phenotyping.

The second part of this thesis (Chapter 5 - 7) describes several studies contributing to our knowledge on SCFA functioning and handling. As SCFA availability declines from the proximal to the distal part of the colon, we investigated in Chapter $\mathbf{5}$ if expression of the SCFA receptors GPR41, GPR43, and GPR109a differed between intestinal locations, i.e. the ileum and several colonic locations. The inclusion of biopsies from different intestinal locations within the same subject provided us with important data on human intestinal SCFA receptor distribution. Moreover, we correlated the SCFA receptor expression with expression of the satiety hormones PYY and GLP-1 in the same biopsies. Furthermore, as applying SCFA enemas, and especially butyrate for treating colonic diseases, is an emerging field, we investigated the inter-organ trafficking of a butyrate enema in Chapter 6 . The experimental set-up during upper abdominal surgery in humans provided the opportunity to administer butyrate rectally with simultaneous blood sampling from the portal vein and a hepatic vein, as well as the radial artery, thereby giving insight in SCFA fluxes over the splanchnic area. In addition, Chapter 7 reviews the current understanding of 
colonic SCFA administration for preventing and treating colonic diseases, and translates the mechanisms proposed by in vivo, in vitro, and animal studies to outcomes of human intervention trials concerning colonic inflammation, carcinogenesis, and mucosal protection and healing.

Finally, Chapter 8 summarizes the main findings of the studies presented in this thesis and discusses new insights, future perspectives, and potential implications for further research. 


\section{REFERENCES}

1. Niess JH, Reinecker HC. Dendritic cells in the recognition of intestinal microbiota. Cellular microbiology 2006;8:558-64.

2. Smith M. The Digestive System. W.B. Saunders Company, 2001.

3. Human Microbiome Project C. Structure, function and diversity of the healthy human microbiome. Nature 2012;486:207-14.

4. Human Microbiome Project C. A framework for human microbiome research. Nature 2012;486:215-21.

5. Li J, Jia H, Cai X, et al. An integrated catalog of reference genes in the human gut microbiome. Nature biotechnology 2014;32:834-41.

6. Backhed F, Ley RE, Sonnenburg JL, et al. Host-bacterial mutualism in the human intestine. Science 2005;307:1915-20.

7. Sommer F, Backhed F. The gut microbiota--masters of host development and physiology. Nature reviews. Microbiology 2013;11:227-38.

8. Swanson HI. Drug Metabolism by the Host and Gut Microbiota: A Partnership or Rivalry? Drug metabolism and disposition: the biological fate of chemicals 2015;43:1499-504.

9. Conly JM, Stein $\mathrm{K}$, Worobetz $\mathrm{L}$, et al. The contribution of vitamin K2 (menaquinones) produced by the intestinal microflora to human nutritional requirements for vitamin $\mathrm{K}$. The American journal of gastroenterology 1994;89:915-23.

10. Kuipers F, Bloks VW, Groen AK. Beyond intestinal soap--bile acids in metabolic control. Nature reviews. Endocrinology 2014;10:488-98.

11. Frank DN, St Amand AL, Feldman RA, et al. Molecular-phylogenetic characterization of microbial community imbalances in human inflammatory bowel diseases. Proceedings of the National Academy of Sciences of the United States of America 2007;104:13780-5.

12. Nagalingam NA, Lynch SV. Role of the microbiota in inflammatory bowel diseases. Inflamm Bowel Dis 2012;18:968-84.

13. Ley RE, Turnbaugh PJ, Klein S, et al. Microbial ecology: human gut microbes associated with obesity. Nature 2006;444:1022-3.

14. Arora T, Backhed F. The gut microbiota and metabolic disease: current understanding and future perspectives. Journal of internal medicine 2016;280:339-49.

15. Dominguez-Bello MG, Costello EK, Contreras M, et al. Delivery mode shapes the acquisition and structure of the initial microbiota across multiple body habitats in newborns. Proceedings of the National Academy of Sciences of the United States of America 2010;107:11971-5.

16. Qin J, Li R, Raes J, et al. A human gut microbial gene catalogue established by metagenomic sequencing. Nature 2010;464:59-65.

17. Rajilic-Stojanovic M, Heilig HG, Molenaar D, et al. Development and application of the human intestinal tract chip, a phylogenetic microarray: analysis of universally conserved phylotypes in the abundant microbiota of young and elderly adults. Environmental microbiology 2009;11:1736-51.

18. Flint HJ. Obesity and the gut microbiota. Journal of clinical gastroenterology 2011;45 Suppl:S128-32.

19. Hamer HM, Jonkers $D$, Venema $K$, et al. Review article: the role of butyrate on colonic function. Aliment Pharmacol Ther 2008;27:104-19. 
20. Bloemen JG, Venema K, van de Poll MC, et al. Short chain fatty acids exchange across the gut and liver in humans measured at surgery. Clin Nutr 2009;28:657-61.

21. Wong JM, de Souza R, Kendall CW, et al. Colonic health: fermentation and short chain fatty acids. J Clin Gastroenterol 2006;40:235-43.

22. Boets E, Gomand SV, Deroover L, et al. Systemic availability and metabolism of colonic-derived short-chain fatty acids in healthy subjects: a stable isotope study. The Journal of physiology 2017;595:541-555.

23. Cummings JH, Macfarlane GT. Colonic microflora: nutrition and health. Nutrition 1997;13:4768.

24. Topping DL, Clifton PM. Short-chain fatty acids and human colonic function: roles of resistant starch and nonstarch polysaccharides. Physiol Rev 2001;81:1031-64.

25. Ahmed R, Segal I, Hassan H. Fermentation of dietary starch in humans. Am J Gastroenterol 2000;95:1017-20.

26. Pokusaeva K, Fitzgerald GF, van Sinderen D. Carbohydrate metabolism in Bifidobacteria. Genes \& nutrition 2011;6:285-306.

27. Flint HJ, Bayer EA, Rincon MT, et al. Polysaccharide utilization by gut bacteria: potential for new insights from genomic analysis. Nature reviews. Microbiology 2008;6:121-31.

28. Cummings JH, Pomare EW, Branch WJ, et al. Short chain fatty acids in human large intestine, portal, hepatic and venous blood. Gut 1987;28:1221-7.

29. Cummings JH. Colonic absorption: the importance of short chain fatty acids in man. Scand J Gastroenterol Suppl 1984;93:89-99.

30. Halestrap AP, Meredith D. The SLC16 gene family-from monocarboxylate transporters (MCTs) to aromatic amino acid transporters and beyond. Pflugers Arch 2004;447:619-28.

31. Harig JM, Ng EK, Dudeja PK, et al. Transport of $n$-butyrate into human colonic luminal membrane vesicles. Am J Physiol 1996;271:G415-22.

32. Miyauchi S, Gopal E, Fei YJ, et al. Functional identification of SLC5A8, a tumor suppressor down-regulated in colon cancer, as a $\mathrm{Na}(+)$-coupled transporter for short-chain fatty acids. J Biol Chem 2004;279:13293-6.

33. Tyagi S, Venugopalakrishnan J, Ramaswamy K, et al. Mechanism of $n$-butyrate uptake in the human proximal colonic basolateral membranes. Am J Physiol Gastrointest Liver Physiol 2002;282:G676-82.

34. Gill RK, Saksena S, Alrefai WA, et al. Expression and membrane localization of MCT isoforms along the length of the human intestine. Am J Physiol Cell Physiol 2005;289:C846-52.

35. Brown AJ, Goldsworthy SM, Barnes AA, et al. The Orphan G protein-coupled receptors GPR41 and GPR43 are activated by propionate and other short chain carboxylic acids. J Biol Chem 2003;278:11312-9.

36. Le Poul E, Loison C, Struyf S, et al. Functional characterization of human receptors for short chain fatty acids and their role in polymorphonuclear cell activation. J Biol Chem 2003;278:25481-9.

37. Thangaraju M, Cresci GA, Liu K, et al. GPR109A is a G-protein-coupled receptor for the bacterial fermentation product butyrate and functions as a tumor suppressor in colon. Cancer Res 2009;69:2826-32.

38. Singh N, Gurav A, Sivaprakasam S, et al. Activation of Gpr109a, receptor for niacin and the commensal metabolite butyrate, suppresses colonic inflammation and carcinogenesis. Immunity 2014;40:128-39. 
39. Liu F, Fu Y, Wei C, et al. The expression of GPR109A, NF-kB and IL-1 beta in peripheral blood leukocytes from patients with type 2 diabetes. Annals of clinical and laboratory science 2014;44:443-8.

40. Karaki S, Tazoe H, Hayashi H, et al. Expression of the short-chain fatty acid receptor, GPR43, in the human colon. Journal of molecular histology 2008;39:135-42.

41. Tazoe H, Otomo Y, Karaki S, et al. Expression of short-chain fatty acid receptor GPR41 in the human colon. Biomedical research 2009;30:149-56.

42. Tazoe H, Otomo Y, Kaji I, et al. Roles of short-chain fatty acids receptors, GPR41 and GPR43 on colonic functions. Journal of physiology and pharmacology : an official journal of the Polish Physiological Society 2008;59 Suppl 2:251-62.

43. Ng M, Fleming T, Robinson $\mathrm{M}$, et al. Global, regional, and national prevalence of overweight and obesity in children and adults during 1980-2013: a systematic analysis for the Global Burden of Disease Study 2013. Lancet 2014;384:766-81.

44. World Health Organization. Fact Sheet Obesity and overweight, 2016.

45. World Health Organization. Obesity: Preventing and managing the global epidemic - report of a WHO consultation, 2000.

46. Kahn SE, Hull RL, Utzschneider KM. Mechanisms linking obesity to insulin resistance and type 2 diabetes. Nature 2006;444:840-6.

47. Backhed F, Ding $\mathrm{H}$, Wang $\mathrm{T}$, et al. The gut microbiota as an environmental factor that regulates fat storage. Proc Natl Acad Sci U S A 2004;101:15718-23.

48. Cox LM, Blaser MJ. Pathways in microbe-induced obesity. Cell metabolism 2013;17:883-94.

49. McNeil NI. The contribution of the large intestine to energy supplies in man. The American journal of clinical nutrition 1984;39:338-42.

50. Chambers ES, Viardot A, Psichas A, et al. Effects of targeted delivery of propionate to the human colon on appetite regulation, body weight maintenance and adiposity in overweight adults. Gut 2015;64:1744-54.

51. Gao Z, Yin J, Zhang J, et al. Butyrate improves insulin sensitivity and increases energy expenditure in mice. Diabetes 2009;58:1509-17.

52. Yamashita $H$, Maruta $H$, Jozuka $M$, et al. Effects of acetate on lipid metabolism in muscles and adipose tissues of type 2 diabetic Otsuka Long-Evans Tokushima Fatty (OLETF) rats. Bioscience, biotechnology, and biochemistry 2009;73:570-6.

53. Cherbut $C$, Ferrier L, Roze $C$, et al. Short-chain fatty acids modify colonic motility through nerves and polypeptide YY release in the rat. The American journal of physiology 1998;275:G1415-22.

54. Cuche G, Cuber JC, Malbert CH. Ileal short-chain fatty acids inhibit gastric motility by a humoral pathway. American journal of physiology. Gastrointestinal and liver physiology 2000;279:G925-30.

55. Freeland KR, Wolever TM. Acute effects of intravenous and rectal acetate on glucagon-like peptide-1, peptide YY, ghrelin, adiponectin and tumour necrosis factor-alpha. The British journal of nutrition 2010;103:460-6.

56. Batterham RL, Cowley MA, Small C), et al. Gut hormone PYY(3-36) physiologically inhibits food intake. Nature 2002;418:650-4.

57. Savage AP, Adrian TE, Carolan G, et al. Effects of peptide YY (PYY) on mouth to caecum intestinal transit time and on the rate of gastric emptying in healthy volunteers. Gut 1987;28:166-70. 
58. Layer P, Holst JJ, Grandt D, et al. Ileal release of glucagon-like peptide-1 (GLP-1). Association with inhibition of gastric acid secretion in humans. Digestive diseases and sciences 1995;40:1074-82.

59. Xiong $Y$, Miyamoto N, Shibata K, et al. Short-chain fatty acids stimulate leptin production in adipocytes through the G protein-coupled receptor GPR41. Proceedings of the National Academy of Sciences of the United States of America 2004;101:1045-50.

60. Soliman M, Kimura K, Ahmed M, et al. Inverse regulation of leptin mRNA expression by shortand long-chain fatty acids in cultured bovine adipocytes. Domestic animal endocrinology 2007:33:400-9.

61. Chambers ES, Morrison DJ, Frost G. Control of appetite and energy intake by SCFA: what are the potential underlying mechanisms? The Proceedings of the Nutrition Society 2015;74:32836.

62. Cani PD, Lecourt E, Dewulf EM, et al. Gut microbiota fermentation of prebiotics increases satietogenic and incretin gut peptide production with consequences for appetite sensation and glucose response after a meal. The American journal of clinical nutrition 2009;90:1236-43.

63. Parnell JA, Reimer RA. Weight loss during oligofructose supplementation is associated with decreased ghrelin and increased peptide $Y Y$ in overweight and obese adults. The American journal of clinical nutrition 2009;89:1751-9.

64. Hardie DG. AMPK--sensing energy while talking to other signaling pathways. Cell metabolism 2014;20:939-52.

65. Sakakibara S, Yamauchi T, Oshima Y, et al. Acetic acid activates hepatic AMPK and reduces hyperglycemia in diabetic KK-A(y) mice. Biochemical and biophysical research communications 2006;344:597-604.

66. den Besten G, Bleeker A, Gerding A, et al. Short-Chain Fatty Acids Protect Against High-Fat Diet-Induced Obesity via a PPARgamma-Dependent Switch From Lipogenesis to Fat Oxidation. Diabetes 2015;64:2398-408.

67. Miles JM, Park YS, Walewicz D, et al. Systemic and forearm triglyceride metabolism: fate of lipoprotein lipase-generated glycerol and free fatty acids. Diabetes 2004;53:521-7.

68. Gray NE, Lam LN, Yang K, et al. Angiopoietin-like 4 (Angptl4) protein is a physiological mediator of intracellular lipolysis in murine adipocytes. The Journal of biological chemistry 2012;287:8444-56.

69. Jocken JW, Blaak EE. Catecholamine-induced lipolysis in adipose tissue and skeletal muscle in obesity. Physiology \& behavior 2008;94:219-30.

70. McQuaid SE, Hodson L, Neville MJ, et al. Downregulation of adipose tissue fatty acid trafficking in obesity: a driver for ectopic fat deposition? Diabetes 2011;60:47-55.

71. Goossens $\mathrm{GH}$. The role of adipose tissue dysfunction in the pathogenesis of obesity-related insulin resistance. Physiology \& behavior 2008;94:206-18.

72. Crouse JR, Gerson CD, DeCarli LM, et al. Role of acetate in the reduction of plasma free fatty acids produced by ethanol in man. Journal of lipid research 1968;9:509-12.

73. Laurent $C$, Simoneau $C$, Marks $L$, et al. Effect of acetate and propionate on fasting hepatic glucose production in humans. European journal of clinical nutrition 1995;49:484-91.

74. Wolever TM, Brighenti F, Royall D, et al. Effect of rectal infusion of short chain fatty acids in human subjects. The American journal of gastroenterology 1989;84:1027-33.

75. Wolever TM, Spadafora P, Eshuis H. Interaction between colonic acetate and propionate in humans. Am J Clin Nutr 1991;53:681-7. 
76. Fernandes J, Vogt J, Wolever TM. Intravenous acetate elicits a greater free fatty acid rebound in normal than hyperinsulinaemic humans. European journal of clinical nutrition 2012;66:1029-34.

77. Fernandes J, Vogt J, Wolever TM. Kinetic model of acetate metabolism in healthy and hyperinsulinaemic humans. European journal of clinical nutrition 2014;68:1067-71.

78. Kimura I, Ozawa K, Inoue D, et al. The gut microbiota suppresses insulin-mediated fat accumulation via the short-chain fatty acid receptor GPR43. Nature communications 2013;4:1829.

79. Ge H, Li X, Weiszmann J, et al. Activation of G protein-coupled receptor 43 in adipocytes leads to inhibition of lipolysis and suppression of plasma free fatty acids. Endocrinology 2008;149:4519-26.

80. Hong $\mathrm{YH}$, Nishimura $\mathrm{Y}$, Hishikawa D, et al. Acetate and propionate short chain fatty acids stimulate adipogenesis via GPCR43. Endocrinology 2005;146:5092-9.

81. Haberland $\mathrm{M}$, Carrer M, Mokalled MH, et al. Redundant control of adipogenesis by histone deacetylases 1 and 2. The Journal of biological chemistry 2010;285:14663-70.

82. Li G, Yao W, Jiang H. Short-chain fatty acids enhance adipocyte differentiation in the stromal vascular fraction of porcine adipose tissue. The Journal of nutrition 2014;144:1887-95.

83. Dewulf EM, Ge Q, Bindels LB, et al. Evaluation of the relationship between GPR43 and adiposity in human. Nutrition \& metabolism 2013;10:11.

84. Al-Lahham SH, Peppelenbosch MP, Roelofsen H, et al. Biological effects of propionic acid in humans; metabolism, potential applications and underlying mechanisms. Biochimica et biophysica acta 2010;1801:1175-83.

85. Kondo T, Kishi M, Fushimi T, et al. Acetic acid upregulates the expression of genes for fatty acid oxidation enzymes in liver to suppress body fat accumulation. Journal of agricultural and food chemistry 2009;57:5982-6.

86. Hardie DG, Ross FA, Hawley SA. AMPK: a nutrient and energy sensor that maintains energy homeostasis. Nature reviews. Molecular cell biology 2012;13:251-62.

87. Akanji AO, Bruce MA, Frayn KN. Effect of acetate infusion on energy expenditure and substrate oxidation rates in non-diabetic and diabetic subjects. European journal of clinical nutrition 1989;43:107-15.

88. Cooke AA, Connaughton RM, Lyons CL, et al. Fatty acids and chronic low grade inflammation associated with obesity and the metabolic syndrome. European journal of pharmacology 2016;785:207-14.

89. Vrieze A, Van Nood E, Holleman F, et al. Transfer of intestinal microbiota from lean donors increases insulin sensitivity in individuals with metabolic syndrome. Gastroenterology 2012;143:913-6 e7.

90. Karlsson FH, Tremaroli V, Nookaew I, et al. Gut metagenome in European women with normal, impaired and diabetic glucose control. Nature 2013;498:99-103.

91. van den Hoek AM, Heijboer AC, Corssmit EP, et al. PYY3-36 reinforces insulin action on glucose disposal in mice fed a high-fat diet. Diabetes 2004;53:1949-52.

92. Boey D, Lin S, Karl T, et al. Peptide YY ablation in mice leads to the development of hyperinsulinaemia and obesity. Diabetologia 2006;49:1360-70.

93. Barrera JG, Sandoval DA, D'Alessio DA, et al. GLP-1 and energy balance: an integrated model of short-term and long-term control. Nature reviews. Endocrinology 2011;7:507-16.

94. Zhou J, Martin RJ, Tulley RT, et al. Dietary resistant starch upregulates total GLP-1 and PYY in a sustained day-long manner through fermentation in rodents. American journal of physiology. Endocrinology and metabolism 2008;295:E1160-6. 
95. Velagapudi VR, Hezaveh R, Reigstad CS, et al. The gut microbiota modulates host energy and lipid metabolism in mice. Journal of lipid research 2010;51:1101-12.

96. Venter $\mathrm{CS}$, Vorster $\mathrm{HH}$, Cummings JH. Effects of dietary propionate on carbohydrate and lipid metabolism in healthy volunteers. The American journal of gastroenterology 1990;85:54953.

97. Todesco T, Rao AV, Bosello O, et al. Propionate lowers blood glucose and alters lipid metabolism in healthy subjects. The American journal of clinical nutrition 1991;54:860-5.

98. Das UN. Is obesity an inflammatory condition? Nutrition 2001;17:953-66.

99. Dandona $\mathrm{P}$, Weinstock $\mathrm{R}$, Thusu K, et al. Tumor necrosis factor-alpha in sera of obese patients: fall with weight loss. The Journal of clinical endocrinology and metabolism 1998;83:2907-10.

100. Rosen ED, Spiegelman BM. What we talk about when we talk about fat. Cell 2014;156:20-44.

101. Bruewer M, Luegering A, Kucharzik T, et al. Proinflammatory cytokines disrupt epithelial barrier function by apoptosis-independent mechanisms. Journal of immunology 2003;171:6164-72.

102. Aguilar-Nascimento JE, Oliveira-Neto JP, Mathie RT, et al. Effect of intraoperative nutritional solutions on perianastomotic colonic mucosa in experimental large bowel obstruction. Dig Dis Sci 1997;42:2581-4.

103. Medzhitov R, Horng T. Transcriptional control of the inflammatory response. Nature reviews. Immunology 2009;9:692-703.

104. Cani PD, Bibiloni R, Knauf C, et al. Changes in gut microbiota control metabolic endotoxemia-induced inflammation in high-fat diet-induced obesity and diabetes in mice. Diabetes 2008:57:1470-81.

105. Cani PD, Possemiers S, Van de Wiele T, et al. Changes in gut microbiota control inflammation in obese mice through a mechanism involving GLP-2-driven improvement of gut permeability. Gut 2009;58:1091-103.

106. Szabo G, Bala S, Petrasek J, et al. Gut-liver axis and sensing microbes. Digestive diseases 2010;28:737-44.

107. Saemann MD, Bohmig GA, Osterreicher $\mathrm{CH}$, et al. Anti-inflammatory effects of sodium butyrate on human monocytes: potent inhibition of IL-12 and up-regulation of IL-10 production. FASEB J 2000;14:2380-2.

108. Tedelind S, Westberg F, Kjerrulf M, et al. Anti-inflammatory properties of the short-chain fatty acids acetate and propionate: a study with relevance to inflammatory bowel disease. World J Gastroenterol 2007;13:2826-32.

109. Russo I, Luciani A, De Cicco P, et al. Butyrate Attenuates Lipopolysaccharide-Induced Inflammation in Intestinal Cells and Crohn's Mucosa through Modulation of Antioxidant Defense Machinery. PLoS One 2012;7:e32841.

110. Segain JP, Raingeard de la Bletiere D, Bourreille A, et al. Butyrate inhibits inflammatory responses through NFkappaB inhibition: implications for Crohn's disease. Gut 2000;47:397403.

111. Ohira H, Fujioka Y, Katagiri C, et al. Butyrate attenuates inflammation and lipolysis generated by the interaction of adipocytes and macrophages. Journal of atherosclerosis and thrombosis 2013;20:425-42.

112. Liu T, Li J, Liu Y, et al. Short-chain fatty acids suppress lipopolysaccharide-induced production of nitric oxide and proinflammatory cytokines through inhibition of NF-kappaB pathway in RAW264.7 cells. Inflammation 2012;35:1676-84.

113. Furusawa Y, Obata Y, Fukuda S, et al. Commensal microbe-derived butyrate induces the differentiation of colonic regulatory T cells. Nature 2013;504:446-50. 
114. Arpaia N, Campbell C, Fan X, et al. Metabolites produced by commensal bacteria promote peripheral regulatory T-cell generation. Nature 2013;504:451-5.

115. Chaudhry A, Rudensky AY. Control of inflammation by integration of environmental cues by regulatory T cells. The Journal of clinical investigation 2013;123:939-44.

116. Peng $L, H e Z$, Chen W, et al. Effects of butyrate on intestinal barrier function in a Caco-2 cell monolayer model of intestinal barrier. Pediatr Res 2007;61:37-41.

117. Suzuki T, Yoshida S, Hara H. Physiological concentrations of short-chain fatty acids immediately suppress colonic epithelial permeability. Br J Nutr 2008;100:297-305.

118. Ferreira TM, Leonel AJ, Melo MA, et al. Oral supplementation of butyrate reduces mucositis and intestinal permeability associated with 5-Fluorouracil administration. Lipids 2012;47:669-78.

119. Russo F, Linsalata M, Clemente C, et al. Inulin-enriched pasta improves intestinal permeability and modifies the circulating levels of zonulin and glucagon-like peptide 2 in healthy young volunteers. Nutr Res 2012;32:940-6.

120. Cox MA, Jackson J, Stanton M, et al. Short-chain fatty acids act as antiinflammatory mediators by regulating prostaglandin E(2) and cytokines. World J Gastroenterol 2009;15:5549-57.

121. Clausen MR, Mortensen PB. Kinetic studies on colonocyte metabolism of short chain fatty acids and glucose in ulcerative colitis. Gut 1995;37:684-9.

122. Hague A, Elder DJ, Hicks DJ, et al. Apoptosis in colorectal tumour cells: induction by the short chain fatty acids butyrate, propionate and acetate and by the bile salt deoxycholate. Int J Cancer 1995;60:400-6.

123. Comalada M, Bailon E, de Haro O, et al. The effects of short-chain fatty acids on colon epithelial proliferation and survival depend on the cellular phenotype. J Cancer Res Clin Oncol 2006;132:487-97.

124. Kim MH, Kang SG, Park JH, et al. Short-chain fatty acids activate GPR41 and GPR43 on intestinal epithelial cells to promote inflammatory responses in mice. Gastroenterology 2013;145:396-406 e1-10.

125. Sterner R, Vidali G, Allfrey VG. Studies of acetylation and deacetylation in high mobility group proteins. Identification of the sites of acetylation in high mobility group proteins 14 and 17 . J Biol Chem 1981;256:8892-5.

126. Correa-Oliveira R, Fachi JL, Vieira A, et al. Regulation of immune cell function by short-chain fatty acids. Clinical \& translational immunology 2016;5:e73.

127. Burger-van Paassen N, Vincent A, Puiman PJ, et al. The regulation of intestinal mucin MUC2 expression by short-chain fatty acids: implications for epithelial protection. Biochem J 2009;420:211-9.

128. Willemsen LE, Koetsier MA, van Deventer SJ, et al. Short chain fatty acids stimulate epithelial mucin 2 expression through differential effects on prostaglandin $E(1)$ and $E(2)$ production by intestinal myofibroblasts. Gut 2003;52:1442-7.

129. Augenlicht L, Shi L, Mariadason J, et al. Repression of MUC2 gene expression by butyrate, a physiological regulator of intestinal cell maturation. Oncogene 2003;22:4983-92.

130. Hatayama H, Iwashita J, Kuwajima A, et al. The short chain fatty acid, butyrate, stimulates MUC2 mucin production in the human colon cancer cell line, LS174T. Biochem Biophys Res Commun 2007;356:599-603.

131. Finnie IA, Dwarakanath AD, Taylor BA, et al. Colonic mucin synthesis is increased by sodium butyrate. Gut 1995;36:93-9.

132. Shimazu T, Hirschey MD, Newman J, et al. Suppression of oxidative stress by beta-hydroxybutyrate, an endogenous histone deacetylase inhibitor. Science 2013;339:211-4. 
133. Abrahamse SL, Pool-Zobel BL, Rechkemmer G. Potential of short chain fatty acids to modulate the induction of DNA damage and changes in the intracellular calcium concentration by oxidative stress in isolated rat distal colon cells. Carcinogenesis 1999;20:629-34.

134. Rosignoli P, Fabiani R, De Bartolomeo A, et al. Protective activity of butyrate on hydrogen peroxide-induced DNA damage in isolated human colonocytes and HT29 tumour cells. Carcinogenesis 2001;22:1675-80.

135. Hinnebusch BF, Meng S, Wu JT, et al. The effects of short-chain fatty acids on human colon cancer cell phenotype are associated with histone hyperacetylation.J Nutr 2002;132:1012-7.

136. Emenaker NJ, Calaf GM, Cox D, et al. Short-chain fatty acids inhibit invasive human colon cancer by modulating UPA, TIMP-1, TIMP-2, mutant p53, BCl-2, Bax, p21 and PCNA protein expression in an in vitro cell culture model. J Nutr 2001;131:3041S-6S.

137. Thangaraju M, Cresci G, Itagaki S, et al. Sodium-coupled transport of the short chain fatty acid butyrate by SLC5A8 and its relevance to colon cancer. J Gastrointest Surg 2008;12:1773-81; discussion 1781-2.

138. Gibson GR, Roberfroid MB. Dietary modulation of the human colonic microbiota: introducing the concept of prebiotics. J Nutr 1995;125:1401-12.

139. Delzenne NM, Cani PD. A place for dietary fibre in the management of the metabolic syndrome. Current opinion in clinical nutrition and metabolic care 2005;8:636-40.

140. Gibson GR, Beatty ER, Wang X, et al. Selective stimulation of bifidobacteria in the human colon by oligofructose and inulin. Gastroenterology 1995;108:975-82.

141. Brighenti F, Casiraghi MC, Canzi E, et al. Effect of consumption of a ready-to-eat breakfast cereal containing inulin on the intestinal milieu and blood lipids in healthy male volunteers. Eur J Clin Nutr 1999;53:726-33.

142. Davis LM, Martinez I, Walter J, et al. Barcoded pyrosequencing reveals that consumption of galactooligosaccharides results in a highly specific bifidogenic response in humans. Plos one 2011;6:e25200.

143. Roberfroid MB, Delzenne NM. Dietary fructans. Annu Rev Nutr 1998;18:117-43.

144. Timm DA, Stewart ML, Hospattankar A, et al. Wheat dextrin, psyllium, and inulin produce distinct fermentation patterns, gas volumes, and short-chain fatty acid profiles in vitro. J Med Food 2010;13:961-6.

145. Kaur A, Rose DJ, Rumpagaporn P, et al. In vitro batch fecal fermentation comparison of gas and short-chain fatty acid production using "slowly fermentable" dietary fibers. Journal of food science 2011;76:H137-42.

146. Wang X, Gibson GR. Effects of the in vitro fermentation of oligofructose and inulin by bacteria growing in the human large intestine. J Appl Bacteriol 1993;75:373-80.

147. Maathuis AJ, van den Heuvel EG, Schoterman MH, et al. Galacto-oligosaccharides have prebiotic activity in a dynamic in vitro colon model using a (13)C-labeling technique. The Journal of nutrition 2012;142:1205-12.

148. Pourghassem Gargari B, Dehghan P, Aliasgharzadeh A, et al. Effects of high performance inulin supplementation on glycemic control and antioxidant status in women with type 2 diabetes. Diabetes Metab J 2013;37:140-8.

149. Letexier D, Diraison F, Beylot M. Addition of inulin to a moderately high-carbohydrate diet reduces hepatic lipogenesis and plasma triacylglycerol concentrations in humans. The American journal of clinical nutrition 2003;77:559-64.

150. Russo F, Chimienti G, Riezzo G, et al. Inulin-enriched pasta affects lipid profile and Lp(a) concentrations in Italian young healthy male volunteers. European journal of nutrition 2008;47:453-9. 
151. Russo F, Clemente C, Linsalata M, et al. Effects of a diet with inulin-enriched pasta on gut peptides and gastric emptying rates in healthy young volunteers. European journal of nutrition 2011;50:271-7.

152. Jackson KG, Taylor GR, Clohessy AM, et al. The effect of the daily intake of inulin on fasting lipid, insulin and glucose concentrations in middle-aged men and women. The British journal of nutrition 1999;82:23-30.

153. Davidson MH, Maki KC. Effects of dietary inulin on serum lipids. J Nutr 1999;129:1474S-7S.

154. Russo F, Riezzo G, Chiloiro M, et al. Metabolic effects of a diet with inulin-enriched pasta in healthy young volunteers. Current pharmaceutical design 2010;16:825-31.

155. Vulevic J, Juric A, Tzortzis G, et al. A mixture of trans-galactooligosaccharides reduces markers of metabolic syndrome and modulates the fecal microbiota and immune function of overweight adults. The Journal of nutrition 2013;143:324-31.

156. Vulevic J, Drakoularakou A, Yaqoob P, et al. Modulation of the fecal microflora profile and immune function by a novel trans-galactooligosaccharide mixture (B-GOS) in healthy elderly volunteers. The American journal of clinical nutrition 2008;88:1438-46. 


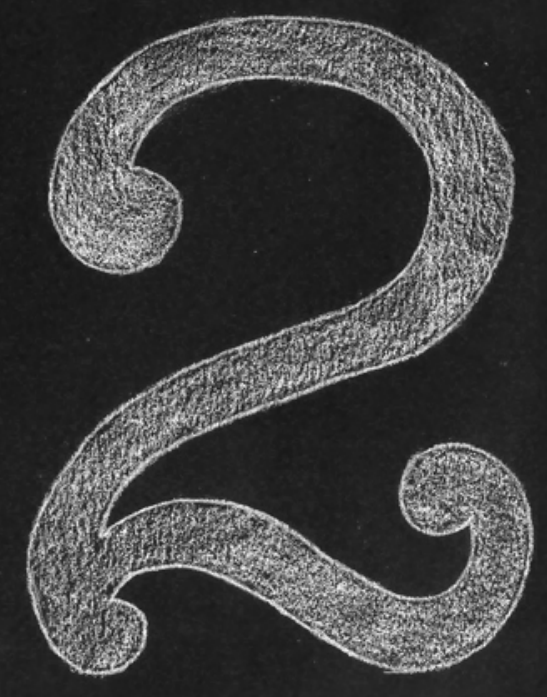




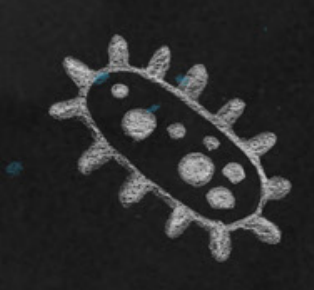

\title{
CHAPTER 2
}

\section{Distal, not proximal, colonic acetate infusions promote fat oxidation and improve metabolic markers in overweight/obese men}

\author{
Christina M. van der Beek*, Emanuel E. Canfora*, Kaatje Lenaerts, \\ Freddy J. Troost, Steven W. M. Olde Damink, Jens J. Holst, Ad A. M. Masclee, \\ Cornelis H. C. Dejong, Ellen E. Blaak
}

*Both authors contributed equally

Clinical Science. 2016;130(22):2073-2082 


\section{ABSTRACT}

Gut microbial-derived short-chain fatty acids (SCFAs) are believed to affect host metabolism and cardiometabolic risk factors. The present study aim was to investigate the effects of proximal and distal colonic infusions with the SCFA acetate on fat oxidation and other metabolic parameters in men. In this randomized, double-blind crossover trial, six overweight/obese men (body mass index (BMI) 25 - 35 kg/m²) underwent two experimental periods: one with distal and one with proximal colonic sodium acetate infusions. A feeding catheter was endoscopically positioned at the beginning of each period and remained in the colon for three consecutive test days, enabling colonic acetate $(100 \mathrm{mmol} / \mathrm{L}$ or 180 $\mathrm{mmol} / \mathrm{L}$ ) or placebo infusion during fasting conditions and after an oral glucose load (postprandial). Fat oxidation and energy expenditure were measured using an opencircuit ventilated hood system and blood samples were repeatedly collected for $2 \mathrm{~h}$ during fasting and postprandial conditions. Distal colonic $180 \mathrm{mmol} / \mathrm{L}$ acetate infusions increased fasting fat oxidation (1.78 \pm 0.28 vs $-0.78 \pm 0.89 \mathrm{~g}$ fat $\left.2 \mathrm{~h}^{-1}, P=0.015\right)$, fasting peptide $\mathrm{YY}$ (PYY, $P=0.01)$ and postprandial glucose and insulin concentrations $(P<0.05)$, and tended to increase fasting plasma acetate $(P=0.069)$ compared with placebo. Distal $100 \mathrm{mmol} / \mathrm{L}$ acetate administration tended to decrease fasting tumor necrosis factor- $a(P=0.067) \mathrm{com}$ pared with placebo. In contrast, proximal colonic acetate infusions showed no effects on substrate metabolism, circulating hormones or inflammatory markers. In conclusion, distal colonic acetate infusions affected whole-body substrate metabolism, with a pronounced increase in fasting fat oxidation and plasma PYY. Modulating colonic acetate may be a nutritional target to treat or prevent metabolic disorders. 


\section{INTRODUCTION}

Accumulating evidence suggests that the gut microbiota and its products may affect host metabolism, body weight, insulin sensitivity, and cardiometabolic risk factors, thereby contributing to the development of obesity and its adverse health consequences. ${ }^{1}$ Among the main products of gut microbiota metabolism are the short-chain fatty acids (SCFAs) acetate, propionate, and butyrate, which are formed in the colon by fermentation of indigestible food components such as dietary fibers. ${ }^{2}$ SCFAs may exert local colonic health effects by changing the $\mathrm{pH}$, regulating local inflammatory responses, and acting as an energy source for colonocytes. ${ }^{3}$ Interestingly, SCFAs, mainly acetate, can enter the bloodstream, ${ }^{4}$ thereby affecting human whole-body energy and substrate metabolism. Additionally, SCFAs might act as signaling molecules between gut microbiota and peripheral tissues, affecting fat oxidation, incretin responses, low-grade systemic inflammation, as well as adipose tissue fat storage; thereby influencing glucose and lipid homeostasis, and insulin sensitivity of the host. ${ }^{2}$

Several in vitro and animal studies indicated that the luminal and circulating most abundant SCFA acetate is an important mediator of substrate and energy metabolism. Rodent-derived data implied that acetate might improve satiety and appetite regulation via secretion of L-cells derived anorexic hormones such as glucagon-like peptide-1 (GLP-1) and peptide YY (PYY), and via central mechanisms..$^{5-7}$ Furthermore, oral acetate administration to mice fed a high-fat diet reduced body weight and improved insulin sensitivity. ${ }^{8}$

Human studies supporting beneficial effects of acetate administration on substrate and energy metabolism are scarce. As early as the late 1960's, a decrease in plasma free fatty acids (FFAs) after a single oral acetate ingestion was observed, pointing to a role of acetate in the modulation of lipolysis. ${ }^{9}$ In hyperinsulinemic women, rectal and intravenous sodium acetate administration has been shown to increase PYY and to suppress the inflammatory marker tumor necrosis factor-a (TNF-a) in plasma. ${ }^{10}$

Data on acute effects of colonic acetate administration on human fat oxidation and metabolic parameters are lacking. Moreover, it is of particular interest whether site of acetate delivery, i.e. proximal or distal colon, differentially affects substrate and energy metabolism. Interestingly, the proximal and distal colon differ in their microbial SCFA production rates, in distribution of the acetate receptor G-protein coupled receptor 43 (GPR43), and in their venous system, as SCFAs partly bypass the liver when absorbed in the distal colon. ${ }^{11-14}$ Therefore, the primary aim of this study was to assess the effect of proximal versus distal colonic acetate infusions on fat oxidation and energy expenditure in overweight and obese healthy men during fasting and postprandial conditions. To this end, we used a unique endoscopic procedure, which allowed us to infuse acetate both proximally and distally in the colon. Secondary outcomes were carbohydrate oxidation, plasma SCFAs, circulating metabolites (glucose, triacylglycerol (TAG), FFAs, free glycerol) 
and hormones (insulin, PYY, GLP-1, leptin, angiopoietin-like protein 4 (ANGPTL4)), and inflammatory markers (TNF-a, interleukin-6 (IL-6), interleukin-8 (IL-8), lipopolysaccharide binding protein (LBP)).

\section{MATERIAL AND METHODS}

\section{Study participants}

Ten healthy Caucasian normoglycemic overweight/obese (BMI 25 - 35 kg/m²) men between 20 - 50 y were recruited between May 2012 and January 2013 from the vicinity of Maastricht, the Netherlands. Six participants finished the trial. Exclusion criteria were: Diabetes mellitus (fasting plasma glucose $\geq 7.0 \mathrm{mmol} / \mathrm{L}$ ), gastroenterological diseases or prior abdominal surgery, cardiovascular diseases, cancer, liver or kidney malfunction, patients with a life expectancy shorter than $5 \mathrm{y}$, men following a hypocaloric diet, use of laxatives, or use of antibiotics, pre- or probiotics in the 3 months prior to start of the study or during the study period. The study was approved by the Medical Ethical Committee of Maastricht University Medical Centre+ (MUMC+) and conducted in accordance with the Declaration of Helsinki (revised version, October 2008, Seoul, South Korea). Written informed consent was obtained from all participants. All authors had access to the study data and reviewed and approved the final manuscript.

\section{Study design}

In this double-blind, placebo-controlled, randomized, crossover study (Figure 2.1A), participants were studied during two experimental periods of 3 consecutive clinical investigation days with at least a 7-day wash-out period in between. During one period a feeding catheter was placed endoscopically in the proximal colon, and during the other period in the distal colon, the order of placement being randomized. In each period, the catheter remained in the colon for 3 consecutive clinical investigational days during which sodium acetate $(100 \mathrm{mmol} / \mathrm{L}$ or $180 \mathrm{mmol} / \mathrm{L}$ dissolved in $120 \mathrm{~mL} 0.9 \% \mathrm{NaCl}$ ) or placebo $(120$ $\mathrm{mL} 0.9 \% \mathrm{NaCl}$ ) was instilled via the catheter on separate days in random order (Figure 2.1A). After an overnight fast, during each clinical investigational day, the participants received colonic infusions during fasting conditions and after an oral glucose load of 75 $\mathrm{g}$ resembling the postprandial state. The order of sodium acetate and placebo solution administration was blinded for both the investigators and participants. The order of catheter placement was randomized but not blinded due to ethical reasons. An independent researcher performed permuted block randomization and assigned participants to interventions. The solutions were isotonic and manufactured by Basic Pharma Technologies B.V. (Geleen, The Netherlands) in accordance to standards of European Good Medical Practice requirements. 
Figure 2.1. Study protocol

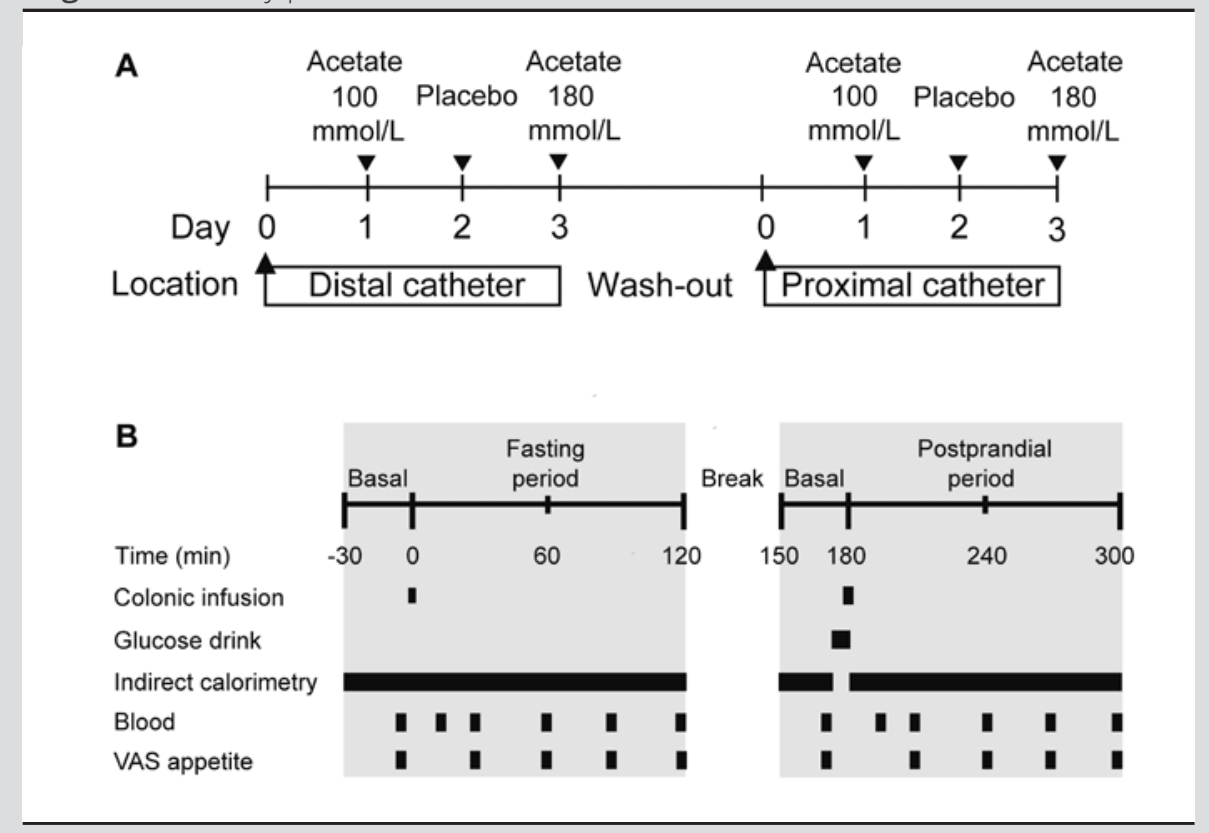

Example of study protocol (A) and test day protocol (B).

\section{Catheter placement}

The day before colonic catheter placement, participants ingested $1 \mathrm{~L}$ of the laxative sodium picosulfate/magnesium citrate (Ferring Pharmaceuticals, Hoofddorp, The Netherlands) combined with 20 mg bisacodyl (Centrafarm, Etten-Leur, The Netherlands) to expel part of colonic content thereby allowing a safe introduction of the endoscope under inspection by the gastroenterologist the following day, but not washing out all microbiota. Participants were not allowed to consume anything after $20.00 \mathrm{~h}$ except for water. The next day, participants came to the hospital and a standard sigmoidoscopy or colonoscopy was performed at the Endoscopy Unit of MUMC+. A feeding catheter (Pur Tube Flocare, Nutricia Medical Devices BV, Schiphol Airport, The Netherlands) was placed either in the ascending colon for proximal infusion or in the descending colon or sigmoid for distal infusion. The catheter was clipped to the colonic mucosa using an endoclip fixation technique (Resolution clip 11 $\mathrm{mm}$, Boston Scientific, Galway, Ireland) to secure its position for three consecutive clinical investigational days. During proximal placement, the participants received intravenous sedation with 2 - $2.5 \mathrm{mg}$ midazolam (Actavis B.V., Baarn, The Netherlands) and the correct position of the catheter was confirmed by standard fluoroscopy. 


\section{Three-day investigation period}

After positioning of the colonic catheter, the participants went to the Metabolic Research Unit of MUMC+ where they stayed for the three-day test period, including the nights. The three consecutive days after the day of catheter placement, the experiments were performed. A standardized diet was given during the entire period. The personalized standardized diets were based on the estimated daily energy expenditure calculated by the Harris Benedict Equation. ${ }^{15}$ The diet consisted of carbohydrates (55 en\%), fat (30 en\%), and protein (15 en\%). Average energy intake was $2355 \pm 165 \mathrm{kcal} / \mathrm{d}$. Participants consumed a meal directly after the test period, had snacks in between and consumed a warm evening meal before $19.30 \mathrm{~h}$. They were fasted overnight. After the third investigational day, the colonic catheter was removed and participants went home (Figure 2.1A).

\section{Clinical investigation days}

Each clinical investigational day consisted of two periods of each $2 \mathrm{~h}$ : a fasting period (t0 - t120) and a subsequent postprandial period (t180 - t300) (Figure 2.1B). Between the periods no measurements took place for $30 \mathrm{~min}$. Prior to the start of the fasting period a cannula was placed in an antecubital vein of the forearm to enable venous blood sampling. Each period was preceded by basal venous blood sampling (at to for the fasting and at t180 for the postprandial period) and measurements of energy expenditure and substrate oxidation for 30 min (from t-30 - t0 before the fasting period and from t150 - t180 before the postprandial period), using an open circuit ventilated hood system (Omnical, Maastricht University Medical Center +, The Netherlands). After baseline measurements colonic acetate or placebo infusion took place, followed by immediate ingestion of the oral glucose load in the postprandial period. The $120 \mathrm{~mL}$ test solutions were manually infused within five min using a syringe via the colonic catheter at a rate of $24 \mathrm{~mL}$ per min at t0 and t180. Subsequently, in both periods, energy expenditure and substrate oxidation were measured during the complete $2 \mathrm{~h}$ period time frames. In addition, venous blood sampling was done at 15, 30, 60, 90, and 120 min after colonic infusions in both periods.

\section{Outcomes}

Primary outcome was the effect of distal and proximal colonic acetate infusions on fat oxidation and energy expenditure during fasting and postprandial conditions measured via indirect calorimetry. The equations of Weir and Frayn ${ }^{16}$ were used to calculate total rate of substrate oxidation and energy expenditure. Secondary outcomes were carbohydrate oxidation, plasma SCFA, circulating metabolites (glucose, TAG, FFAs, free glycerol), hormones (insulin, active PYY, total GLP-1, leptin, ANGPTL4) and inflammatory markers (TNF-a, IL-6, IL-8, LBP).

For the metabolites, blood was collected into ice-cold EDTA tubes (0.2M EDTA (Sigma, Dorset, UK)). For GLP-1 analysis, blood was collected in a 2 mL EDTA tube containing 
$20 \mu \mathrm{L}$ of dipeptidyl peptidase-IV inhibitor (Millipore, Darmstadt, Germany). For PYY analysis, blood was collected in a $2 \mathrm{~mL}$ aprotinin tube containing $20 \mu \mathrm{L}$ of dipeptidyl peptidase-IV inhibitor. The samples were centrifuged at 3,500 g, $4{ }^{\circ} \mathrm{C}$ for $10 \mathrm{~min}$, plasma was aliquoted and directly snap-frozen in liquid nitrogen and stored at $-80{ }^{\circ} \mathrm{C}$ until analysis. FFAs, TAG and glucose were measured with enzymatic assays on an automated spectrophotometer (ABX Pentra 400 autoanalyser, Horiba ABX, Montpellier, France). Free glycerol was measured after precipitation with an enzymatic assay (EnzytecTM Glycerol, Roche Biopharm, Switzerland) automated on a Cobas Fara spectrophotometric autoanalyser (Roche Diagnostics, Basel, Switzerland). The concentrations of insulin, leptin, and PYY were determined with radioimmunoassay (RIA) kits (Human Insulin specific RIA, Human Leptin RIA, Human PYY (3-36) RIA, Millipore Corporation, MA, USA). IL-6, IL-8, and TNF-a were determined with an enzyme-linked immunosorbent assay (ELISA) kit (Human Prolnflammatory II 4-Plex Ultra-Sensitive Kit, Meso Scale Diagnostics, MD, USA). The ANGPTL4 concentrations were determined with ELISA (Human ANGPTL4 ELISA Kit, RayBiotech, Norcross, USA). Samples were assayed for total GLP-1 immunoreactivity using an antiserum, which reacts equally with intact GLP-1 and the primary (N-terminally truncated) metabolite as previously described. ${ }^{17}$ For LBP detection, plates (Microlon 600 high binding, Greiner Bio-One, Alphen aan de Rijn, The Netherlands) were coated with polyclonal anti-human LBP. Diluted plasma samples (1:5000) and a standard dilution series with rLBP were added to the plate. Detection occurred with a biotinylated polyclonal rabbit anti-human LBP IgG, followed by peroxidase-conjugated streptavidin and substrate. Deproteinization and preparation of plasma samples for SCFA analysis was performed as reported before, ${ }^{18}$ using a liquid chromatography system combined with mass spectrometry.

\section{Statistical analysis}

Values are expressed as mean \pm SEM. Responses after distal and proximal colonic administration during fasting (t0 - t120) and postprandial (t180 - t300) conditions are expressed as incremental area under the curve (iAUC), which were calculated by the trapezoid method and checked for normal distribution by a Kolmogorov-Smirnov test. Differences in fasting and postprandial iAUC between acetate and placebo infusions were analyzed using a linear mixed model for repeated measures. Intervention, period (catheter placement order) and subperiod (intervention order) were set as fixed factors and participants were set as random factor. Pairwise comparisons were performed with Fisher's Least Significant Difference (LSD) test. No carry-over effect was apparent to previous clinical investigational day; therefore, previous intervention was not included in the model. iAUC not shown as values or figures did not present statistically significant differences. All statistics were performed using SPSS 22.0 for Mac (Chicago, IL, USA) and P<0.05 (two-sided P-value) was considered statistically significant. A P-value smaller than 0.10 was regarded as a trend. 


\section{Power calculation}

To detect a 20\% difference in the primary outcome parameter fat oxidation between the acetate treatments and placebo, the required sample size was 6 to achieve a power of $80 \%$ at a 2 -sided a-level of 0.05 . Assuming a drop-out of $40 \%$ we totally included 10 subjects.

\section{RESULTS}

Six participants completed all six experimental days and were included in the final analysis. Four participants were excluded after two failed attempts to clip the catheter to the colonic mucosa on two separate days. Mean body mass index (BMI) of the participants was $31.0 \pm$ $1.5 \mathrm{~kg} / \mathrm{m}^{2}$ and they had a mean fasting glucose of $5.1 \pm 0.1 \mathrm{mmol} / \mathrm{L}$ (Table 2.1). No adverse events were reported. All basal values of the reported variables were comparable between the different experimental conditions.

Table 2.1. Study participants' characteristics $(n=6)$

\begin{tabular}{lr}
\hline Variable & Mean \pm SEM \\
\hline Age $(\mathrm{y})$ & $30.2 \pm 4.7$ \\
$\mathrm{BMI}\left(\mathrm{kg} / \mathrm{m}^{2}\right)$ & $31.0 \pm 1.5$ \\
Waist circumference $(\mathrm{cm})$ & $108.0 \pm 4.9$ \\
Systolic blood pressure $(\mathrm{mmHg})$ & $126.3 \pm 3.5$ \\
Diastolic blood pressure $(\mathrm{mmHg})$ & $83.7 \pm 2.1$ \\
$\mathrm{HbA}_{1 \mathrm{C}}(\%)$ & $5.3 \pm 0.1$ \\
Fasting glucose $(\mathrm{mmol} / \mathrm{L})$ & $5.1 \pm 0.1$ \\
\hline
\end{tabular}

Abbreviations: BMI, Body Mass Index; $\mathrm{HbA}_{1 C^{\prime}}$ Hemoglobin $\mathrm{A}_{1 \mathrm{C}}$

\section{Fat oxidation, energy expenditure, and carbohydrate oxidation}

Fasting fat oxidation was higher after distal $180 \mathrm{mmol} / \mathrm{L}$ acetate administration $(1.78 \pm 0.28$ vs $-0.78 \pm 0.89 \mathrm{~g}$ fat $\left.2 \mathrm{~h}^{-1}, P=0.015\right)$ and tended to be higher after distal $100 \mathrm{mmol} / \mathrm{L}$ acetate infusion (0.88 \pm 0.45 vs $-0.78 \pm 0.89 \mathrm{~g}$ fat $2 \mathrm{~h}^{-1}, P=0.059$ ) compared with placebo (Figure 2.2A and 2.2C). Fasting fat oxidation was not significantly different between interventions after proximal acetate infusion (Figure 2.2B). Postprandial fat oxidation decreased in all intervention groups. However, no significant differences between the groups neither after distal nor after proximal colonic infusions were detected.

Postprandial energy expenditure increased in all intervention groups. However, no significant differences between interventions in both fasting and postprandial conditions were observed (Figure 2.2D and 2.2E). 
No significant differences in fasting carbohydrate oxidation were observed after distal and proximal administration. Postprandial carbohydrate oxidation increased in all interventions without significant differences between the intervention groups neither after distal nor after proximal colonic infusions (Figure 2.2F and 2.2G).

Figure 2.2. Energy expenditure and substrate oxidation
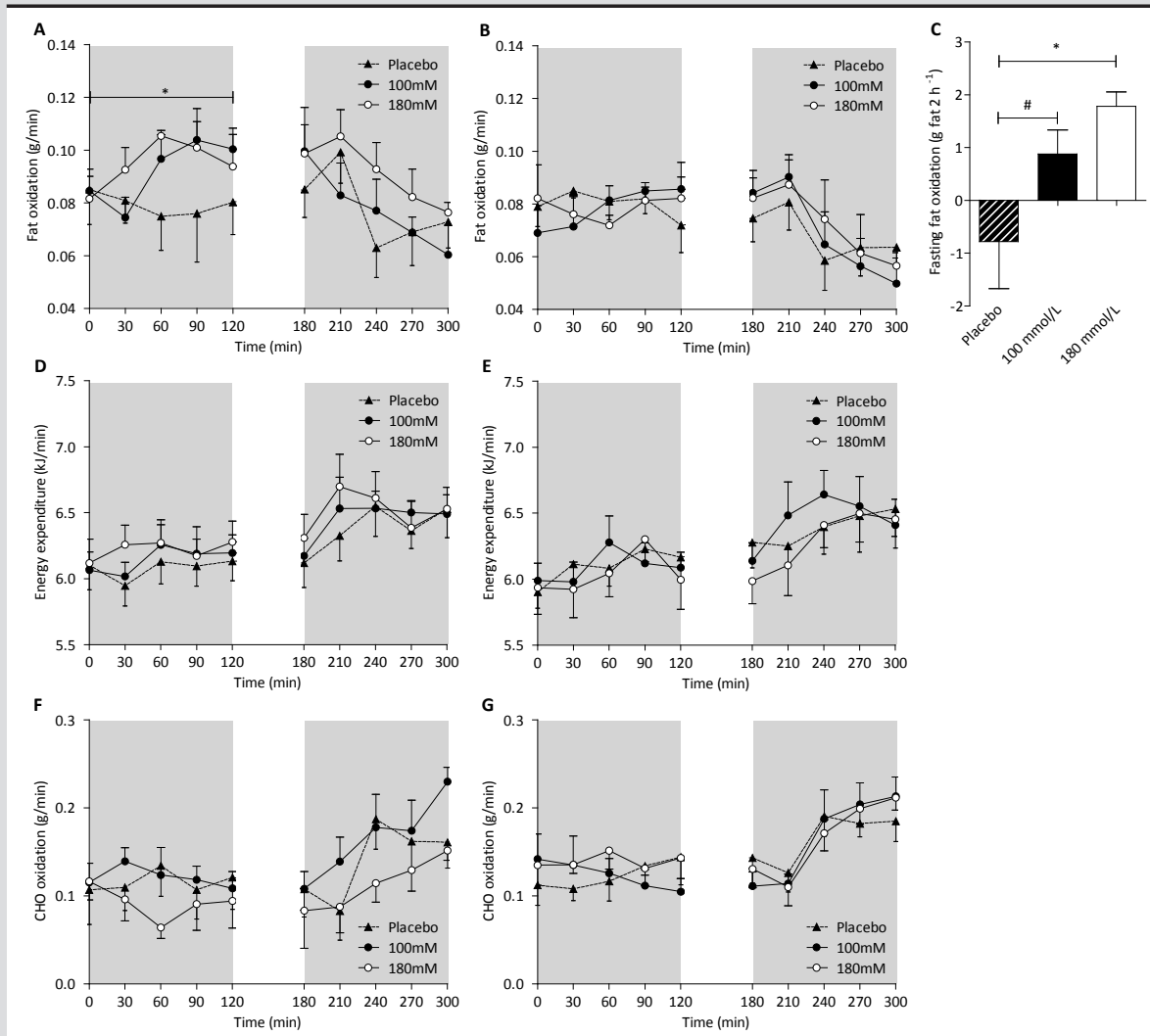

Fasting (t0 - t120) and postprandial (t180 - t300) fat oxidation after distal (A) and proximal (B) colonic infusions. IAUC for fasting (t0 - t120) fat oxidation following distal colonic acetate infusions (C). Overall intervention effect for fasting fat oxidation after distal acetate, $P=0.040$ (180 $\mathrm{mmol} / \mathrm{L}$ vs placebo, $P=0.015 ; 100 \mathrm{mmol} / \mathrm{L}$ vs placebo, $P=0.059)$.

Fasting (t0 - t120) and postprandial (t180- t300) energy expenditure after distal (D) and proximal (E) colonic infusions. No significant differences between interventions.

Fasting (t0 - t120) and postprandial (t180 - t300) carbohydrate oxidation after distal (F) and proximal (G) colonic infusions. No significant differences between interventions.

Values are means \pm SEMs $(n=6)$. * $P<0.05$, \# $P<0.1$. Abbreviations: $\mathrm{CHO}$, Carbohydrate. 


\section{Plasma acetate, propionate and butyrate concentrations}

Fasting plasma acetate concentrations tended to be higher after distal $180 \mathrm{mmol} / \mathrm{L}$ acetate administration, as compared to placebo (-833 \pm 663 vs $1344 \pm 1036 \mu \mathrm{mol} / \mathrm{L} 2 \mathrm{~h}^{-1}, P=0.069$; Figure 2.3A and 2.3C). No significant differences between interventions were found during the fasting and postprandial period after proximal colonic infusions (Figure 2.3B).

Plasma propionate and butyrate concentrations were not significantly affected after all intervention periods and administration sides (Supplemental Figure 2.1).

\section{Plasma concentrations of metabolites, hormones and inflammatory markers}

Postprandial glucose concentrations were higher after $180 \mathrm{mmol} / \mathrm{L}$ acetate administration in the distal colon compared with placebo (303 \pm 42 vs $228 \pm 38 \mathrm{mmol} / \mathrm{L}^{2} \mathrm{~h}^{-1}, P=0.004$ Figure 2.3D and 2.3F). In line, postprandial insulin concentrations were higher after distal administration of $180 \mathrm{mmol} / \mathrm{L}$ acetate as compared to placebo (6759 \pm 1281 vs $5578 \pm$ $1180 \mathrm{mU} / \mathrm{L} 2 \mathrm{~h}^{-1}, \mathrm{P}=0.016$; Figure 2.3G and 2.31). No differences in fasting glucose or insulin concentrations were observed when acetate was distally infused (Figure 2.3D and 2.3G). No effects of proximally infused acetate on circulating glucose and insulin concentrations were found (Figure 2.3E and 2.3H).

PYY was higher in the fasting period after distal $180 \mathrm{mmol} / \mathrm{L}$ acetate infusion as compared to placebo (1976 \pm 391 vs $0 \pm 535$ ng/L 2 h-1, P=0.009; Figure 2.4A and 2.4C). Proximally administered acetate did not significantly affect PYY plasma concentrations (Figure 2.4B). Fasting GLP-1, as well as postprandial increase in GLP-1 concentrations were not significantly affected by the administered acetate after proximal or distal colonic acetate infusions (Figure 2.4D and 2.4E).

TNF-a plasma concentrations tended to be lower in the fasting state after distal administration of $100 \mathrm{mmol} / \mathrm{L}$ acetate when compared with placebo $(-21.7 \pm 9.8 \mathrm{vs}-2.1 \pm 8.8 \mathrm{ng} / \mathrm{L}$ $2 \mathrm{~h}^{-1}, \mathrm{P}=0.067$; Figure 2.4F and 2.4H). The lowered plasma TNF-a concentration was not significantly different in the postprandial state. Proximal administration of acetate did not influence TNF-a concentrations (Figure 2.4G). Plasma IL-6, IL-8 and LBP concentrations were not significantly influenced by the administered acetate, neither after distal nor after proximal administration (Supplemental Figure 2.2).

No significant differences in circulating TAG, FFAs, and free glycerol concentrations were observed between interventions neither in the fasting nor in the postprandial period. Postprandially, TAG, FFAs, and free glycerol concentrations decreased in all intervention groups with no significant differences between groups (Supplemental Figure 2.3).

No significant differences in plasma leptin and ANGPTL4 concentrations were observed between interventions neither in the fasting nor in the postprandial period (Supplemental Figure 2.4). 
Figure 2.3. Plasma concentrations of acetate, glucose, and insulin
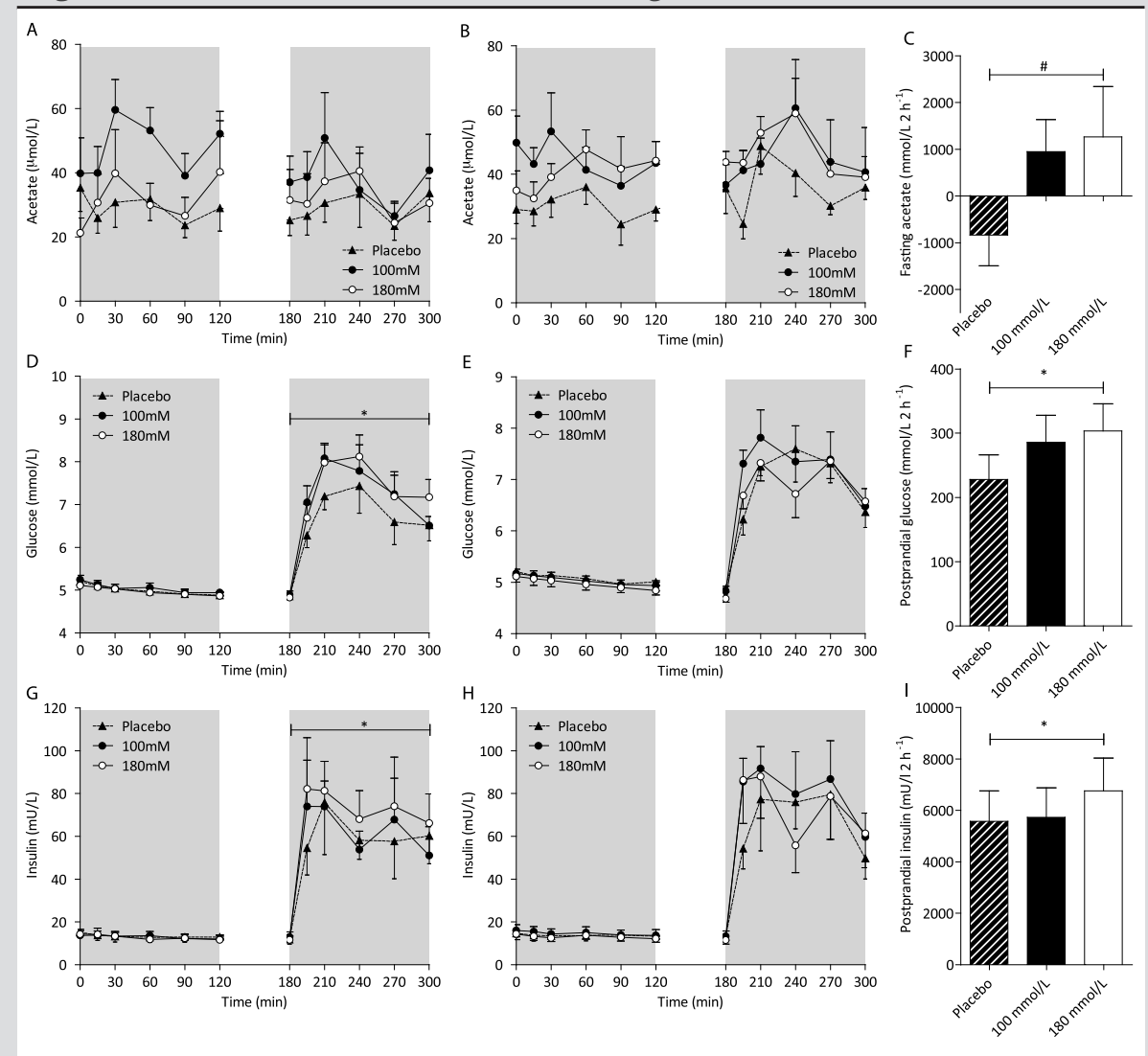

Fasting (t0 - t120) and postprandial (t180 - t300) plasma acetate concentrations after distal (A) and proximal (B) colonic acetate infusions. iAUC for fasting (t0 - t120) plasma acetate following distal colonic infusion (C). Overall intervention effect for fasting plasma acetate after distal infusions $P=0.157$ ( $180 \mathrm{mmol} / \mathrm{L}$ vs placebo, $P=0.069$ ).

Fasting (t0 - t120) and postprandial (t180 - t300) plasma glucose after distal (D) and proximal (E) colonic acetate infusions. iAUC for postprandial (t180 - t300) plasma glucose following distal colonic acetate infusion (F). Overall intervention effect for postprandial glucose after distal acetate, $P=0.010$ (180 $\mathrm{mmol} / \mathrm{L}$ vs placebo, $P=0.004)$.

Fasting (t0 - t120) and postprandial (t180 - t300) plasma insulin after distal (G) and proximal (H) colonic acetate infusions. iAUC for postprandial (t180 - t300) plasma insulin following distal colonic acetate infusions (I). Overall intervention effect for postprandial insulin after distal acetate $P=0.028(180 \mathrm{mmol} / \mathrm{L}$ vs placebo, $P=0.016)$.

Values are means $\pm \operatorname{SEMS~}(n=6)$. ${ }^{*} P<0.05$, \# $P<0.1$. 
Figure 2.4. Plasma concentrations of PYY, GLP-1, and TNF- $a$
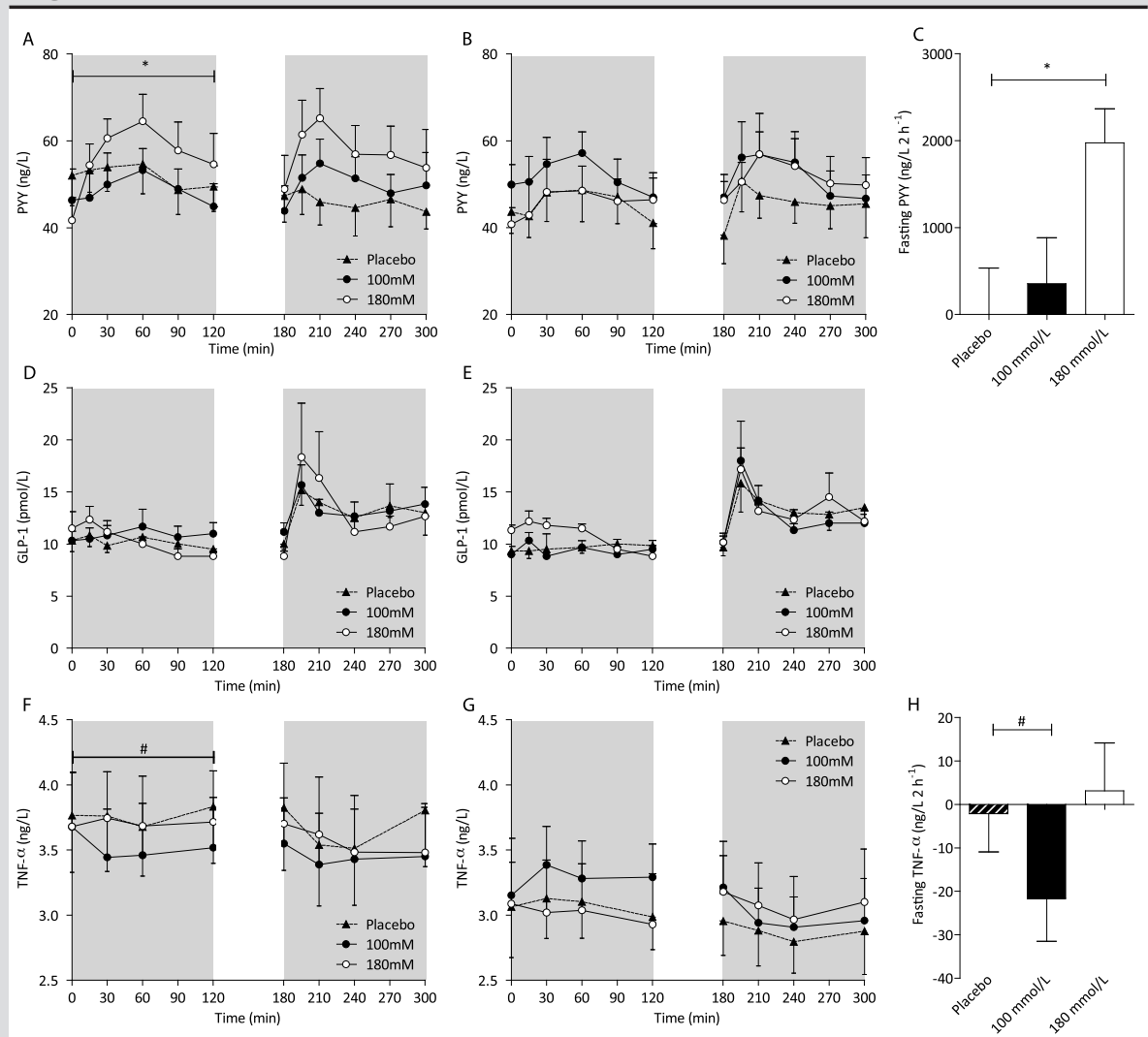

Fasting (t0 - t120) and postprandial (t180 - t300) plasma PYY after distal (A) and proximal (B) colonic acetate infusions. IAUC for fasting (t0 - t120) plasma PYY following distal colonic infusion (C). Overall intervention effect for fasting PYY after distal acetate, $P=0.019(180 \mathrm{mmol} / \mathrm{L}$ vs placebo, $P=0.009$ ).

Fasting (t0 - t120) and postprandial (t180 - t300) plasma GLP-1 after distal (D) and proximal (E) colonic acetate infusions. No significant differences between interventions.

Fasting (t0 - t120) and postprandial (t180 - t300) plasma TNF-a after distal (F) and proximal (G) colonic acetate infusions. iAUC for fasting (t0 - t120) plasma TNF-a following distal colonic acetate infusions $(\mathbf{H})$. Overall intervention effect for fasting TNF-a after distal acetate $P=0.089$ (100 $\mathrm{mmol} / \mathrm{L}$ vs placebo, $P=0.067$ ).

Values are means $\pm \operatorname{SEMs}(n=6) .{ }^{*} P<0.05, \# P<0.1$. 


\section{DISCUSSION}

In the present randomized, placebo-controlled, double-blind crossover study, we assessed the effect of sodium acetate $(180 \mathrm{mmol} / \mathrm{L}$ and $100 \mathrm{mmol} / \mathrm{L})$ and placebo infusions in the proximal and distal part of the colon on fat oxidation and metabolic parameters in overweight and obese normoglycemic men. We demonstrated that distal colonic acetate infusions pronouncedly increased fasting fat oxidation with more than 25\% (180 mmol/L vs placebo) and increased fasting plasma PYY concentrations. In addition, administration of distal colonic acetate slightly raised fasting circulating acetate, and postprandial glucose and insulin concentrations, as well as it tended to decrease TNF-a concentrations. Proximal colonic acetate instillation did not result in significant metabolic effects. This is the first human intervention study that compared acetate administration in different colonic regions and showed that putatively physiologically relevant distal colonic acetate concentrations ${ }^{19-21}$ promote fat oxidation and alter metabolic parameters in overweight and obese volunteers.

The used amounts and concentrations of acetate in the present study can putatively be achieved ingesting a high fiber diet. The only human data available showed basal total SCFA concentrations of $123 \pm 12$ (mean \pm standard error) $\mathrm{mmol} / \mathrm{L}$ in the proximal and $100 \pm 30 \mathrm{mmol} / \mathrm{L}$ in the distal colon in sudden death victims. ${ }^{21}$ These data already showed that SCFA concentrations are highly variable. The production rates of SCFAs in the colon strongly depend on the nutritional status, amount of complex foods in the diet, gut microbial composition, and colonic transit time. ${ }^{22-24}$ In vitro studies indicate that a daily amount of approximately 400 - 800 mmol SCFAs can be produced by ingesting a high-fiber diet, assuming that $10 \mathrm{~g}$ of dietary fiber fermentation yield $\sim 100 \mathrm{mmol}$ SCFAs. ${ }^{23,25}$ These estimates agree with the results on in vitro fermentation of ileal effluents from patients with an ileostomy, which produced 120 - 420 mmol acetate per day, depending upon the type and amount of complex carbohydrates in their diet. ${ }^{25}$

In humans, microbial SCFA production seems to peak in the cecum and proximal part of the colon, while a smaller amount of SCFAs are produced in the distal colon. ${ }^{21}$ Interestingly, the present study showed that metabolic effects were specifically found after distal acetate infusion, but not after proximal infusion. This could be explained by several factors. First, SCFAs delivered in the distal colon partly bypass the liver via the rectal venous plexus, which drains into the inferior vena cava, thereby directly reaching the systemic circulation. ${ }^{11}$ Indeed, plasma acetate concentrations tended to be higher with distal, but not proximal, colonic infusion (Figure 2.3A, 2.3B, and 2.3C), from which it might be speculated that an increase in systemic acetate may be required for the observed metabolic effects. SCFAs delivered in the proximal colon enter the superior mesenteric vein, which drains into the portal vein. Secondly, the SCFA receptor GPR43 is highly expressed in the 
distal colon, possibly contributing to the metabolic differences between proximal and distal infusions. ${ }^{12}$ Thirdly, microbiota composition and its activity differ between proximal and distal colonic locations. 13,14 This might have resulted in higher microbial utilization or conversation rate of the infused sodium acetate to other metabolites such as butyrate in the proximal colon and consequently less acetate absorption and putative acetate-induced metabolic effects.

In the present study, we demonstrated that distal, but not proximal, colonic sodium acetate infusions enhanced our major outcome parameter whole-body fat oxidation. Intracellular acetate can be converted by the enzyme acetyl-CoA synthetase into acetylCoA, which can directly enter the citric acid cycle and thereby delivers energy to the host. Therefore, it can be questioned if the found effect on fat oxidation is not due to the oxidation of sodium acetate per se, rather than the oxidation of endogenous lipids. However, when $100 \%$ of the colonically infused sodium acetate would have been converted into acetyl-CoA, this would only explain $\sim 28 \%$ of the found increase in fat oxidation ( $180 \mathrm{mmol} / \mathrm{L}$ sodium acetate vs placebo). However, it is likely that not $100 \%$ of the acetate is completely oxidized, based on the fact that at least $60 \%$ of acetate absorption is by simple diffusion of protonated acetate 26,27 and that a kinetic study showed that only 30 - 50\% of rectally administered ${ }^{13} \mathrm{C}$-acetate is oxidized after $4 \mathrm{~h} .{ }^{28}$ This indicates that the estimated $28 \%$ is a large overestimation. For a detailed calculation, please see supplemental methods. Hence, other mechanisms are responsible for the acetate-induced increase in fat oxidation after distal colonic acetate infusion. Rodent-derived data indicate that acetate may activate adipose tissue and skeletal muscle $5^{\prime}$ adenosine monophosphate-activated protein kinase (AMPK), possibly mediated through an acetate-induced increased AMP/ ATP ratio and upregulation of peroxisome proliferator-activated receptor (PPAR) target genes, ${ }^{8,29,30}$ which might stimulate the oxidative metabolism in these tissues. ${ }^{31}$ Furthermore, we observed an elevation in fasting plasma PYY after distal colonic infusion. Studies performed in mice and preliminary human data suggest that an increase in circulating PYY is responsible for a shift in substrate utilization towards fat oxidation. ${ }^{32,33}$ PYY might also acutely increase glucose uptake from the gut, ${ }^{34}$ which might explain the slightly elevated postprandial plasma glucose and insulin after distal infusion.

TNF-a, which tended to be reduced after distal acetate infusion in our study, has been linked to a state of low-grade inflammation in obesity and insulin resistance..$^{35}$ Our data are consistent with a reduced TNF-a release from neutrophils after in vitro acetate treatment. ${ }^{36}$ Most cytokines, including TNF-a, involved in inducing and/or maintaining inflammation are controlled by transcription factor NF-kB, and it can be hypothesized that suppressing NF-kB by acetate can cause suppression of these pro-inflammatory markers. ${ }^{37}$ Nevertheless, we only demonstrated a tendency towards decreased TNF-a concentrations after distal colonic acetate infusion with a $100 \mathrm{mmol} / \mathrm{L}$, but not with the $180 \mathrm{mmol} / \mathrm{L}$. The reason for this is not entirely clear and the physiological relevance of this finding remains to be confirmed in future studies. 
The increase in PYY and the decrease in TNF-a concentrations are in line with a human study in six obese hyperinsulinemic women. ${ }^{10}$ They showed that rectal infusions with acetate increased serum acetate and plasma PYY, and intravenous infusions of acetate decreased plasma TNF-a levels compared with saline infusions. Comparable to the present findings and other studies in humans ${ }^{38,39}$ circulating acetate concentrations peaked between 30 - 60 min after distal colonic acetate administration. We extended previous data showing pronounced effects on human fat oxidation and the differential metabolic effect between proximal and distal colonic infusion. In addition, we investigated acetateinduced effects during fasting and postprandial conditions for a prolonged timeframe of $2 \mathrm{~h}$.

We are aware that the present study has limitations. Most are due to the complex study design and the invasive nature of the study using colonic catheters during prolonged periods. First, inherent to the design, the use of laxation prior to colonoscopy is not ideal. It is possible that the microbiota changed after laxation. ${ }^{40}$ However, in vitro studies showed that the major groups of microbiota are stable at different transit times..$^{41}$ Importantly, we tried to minimize this possible microbial change by using only $1 / 4^{\text {th }}$ of laxation product normally used for diagnostic colonoscopies. Due to the length and small width of the catheters, we were not able to sample fecal material during the periods. Second, because the catheter stayed in the colon for three consecutive days, we had a washout period of about $16 \mathrm{~h}$ between test days. SCFAs are, however, rapidly absorbed in the colon. ${ }^{42}$ Indeed, we observed plasma SCFA levels returning to baseline at the beginning of the next test day, suggesting a normalization of SCFA concentrations. Third, due to the invasive protocol, we only included six volunteers in this study. We calculated, however, that this sample size is adequate for detecting differences on fat oxidation, our primary outcome.

This study has important clinical perspectives. The substantial increase in fat oxidation after colonic acetate supplementation may have substantial future potential if maintained during chronic treatment. An increase in fat oxidation can decrease fat storage in nonadipose tissue, such as skeletal muscle or liver tissue. A decrease in ectopic fat and bioactive lipid metabolites has been associated with improved insulin resistance, an important target in the treatment of metabolic diseases. ${ }^{43}$ Further studies should clarify the underlying mechanisms involved in the acetate-induced upregulated oxidative capacity and investigate whether similar effects may be obtained by acetogenic dietary fibers.

In conclusion, this study showed for the first time, that distal, but not proximal, colonically administered acetate modulates whole-body substrate metabolism, with a pronounced increase in fasting fat oxidation and in the satiety-stimulating hormone PYY. This suggests that modulating colonic acetate via i.e. slow-fermentable acetogenic foods might be a nutritional or therapeutic target to treat or prevent chronic metabolic diseases. 


\section{REFERENCES}

1. Diamant M, Blaak E, De Vos W. Do nutrient-gut-microbiota interactions play a role in human obesity, insulin resistance and type 2 diabetes? obesity reviews 2011;12:272-281.

2. Canfora EE, Jocken JW, Blaak EE. Short-chain fatty acids in control of body weight and insulin sensitivity. Nat Rev Endocrinol 2015.

3. Hamer HM, Jonkers D, Venema K, et al. Review article: the role of butyrate on colonic function. Aliment Pharmacol Ther 2008;27:104-19.

4. Bloemen JG, Venema K, van de Poll MC, et al. Short chain fatty acids exchange across the gut and liver in humans measured at surgery. Clin Nutr 2009;28:657-61.

5. Frost G, Sleeth ML, Sahuri-Arisoylu M, et al. The short-chain fatty acid acetate reduces appetite via a central homeostatic mechanism. Nature communications 2014;5.

6. Tolhurst G, Heffron H, Lam YS, et al. Short-chain fatty acids stimulate glucagon-like peptide-1 secretion via the G-protein-coupled receptor FFAR2. Diabetes 2012;61:364-71.

7. Psichas A, Zac-Varghese S, Murphy K, et al. Short chain fatty acids stimulate the release of gut hormone peptide YY from human primary enteroendocrine $L$ cells, In Proceedings of The Physiological Society, 27, PC331, 2012.

8. den Besten G, Bleeker A, Gerding A, et al. Short-Chain Fatty Acids protect against High-Fat Diet-Induced Obesity via a PPARy-dependent switch from lipogenesis to fat oxidation. Diabetes 2015:db141213.

9. Crouse JR, Gerson CD, DeCarli LM, et al. Role of acetate in the reduction of plasma free fatty acids produced by ethanol in man. Journal of lipid research 1968;9:509-512.

10. Freeland KR, Wolever T. Acute effects of intravenous and rectal acetate on glucagon-like peptide-1, peptide YY, ghrelin, adiponectin and tumour necrosis factor-alpha. British Journal of Nutrition 2010;103:460-466.

11. Moeinian M, Ghasemi-Niri SF, Mozaffari S, et al. Beneficial effect of butyrate, Lactobacillus casei and L-carnitine combination in preference to each in experimental colitis. World journal of gastroenterology 2014;20:10876-85.

12. Kaji I, Karaki S, Tanaka R, et al. Density distribution of free fatty acid receptor 2 (FFA2)-expressing and GLP-1-producing enteroendocrine L cells in human and rat lower intestine, and increased cell numbers after ingestion of fructo-oligosaccharide. Journal of molecular histology 2011;42:27-38.

13. Alander $\mathrm{M}$, De Smet I, Nollet $\mathrm{L}$, et al. The effect of probiotic strains on the microbiota of the Simulator of the Human Intestinal Microbial Ecosystem (SHIME). International journal of food microbiology 1999;46:71-9.

14. Wang M, Ahrne S, Jeppsson B, et al. Comparison of bacterial diversity along the human intestinal tract by direct cloning and sequencing of $16 \mathrm{~S}$ rRNA genes. FEMS microbiology ecology 2005;54:219-31.

15. Harris JA, Benedict FG. A biometric study of human basal metabolism. Proceedings of the National Academy of Sciences of the United States of America 1918;4:370.

16. Frayn K. Calculation of substrate oxidation rates in vivo from gaseous exchange. Journal of Applied Physiology 1983;55:628.

17. Orskov C, Rabenhoj L, Wettergren A, et al. Tissue and plasma concentrations of amidated and glycine-extended glucagon-like peptide I in humans. Diabetes 1994;43:535-9. 
18. van Eijk HM, Bloemen JG, Dejong CH. Application of liquid chromatography-mass spectrometry to measure short chain fatty acids in blood. Journal of chromatography. B, Analytical technologies in the biomedical and life sciences 2009;877:719-24.

19. CummingsJH, Macfarlane GT. Colonic microflora: nutrition and health. Nutrition 1997;13:476-8.

20. Andoh A, Tsujikawa T, Fujiyama Y. Role of dietary fiber and short-chain fatty acids in the colon. Curr Pharm Des 2003;9:347-58.

21. Cummings J, Pomare E, Branch W, et al. Short chain fatty acids in human large intestine, portal, hepatic and venous blood. Gut 1987;28:1221.

22. Venema K. Microbial metabolites produced by the colonic microbiota as drivers for immunomodulation in the host. The FASEB Journal 2013;27:643.12.

23. Pylkas AM, Juneja LR, Slavin JL. Comparison of different fibers for in vitro production of short chain fatty acids by intestinal microflora. Journal of medicinal food 2005;8:113-116.

24. El Oufir L, Barry J, Flourie B, et al. Relationships between transit time in man and in vitro fermentation of dietary fiber by fecal bacteria. European journal of clinical nutrition 2000;54:603-609.

25. McBurney M, Thompson L, Cuff D, et al. Comparison of ileal effluents, dietary fibers, and whole foods in predicting the physiological importance of colonic fermentation. The American journal of gastroenterology 1988;83:536.

26. Wong JM, de Souza R, Kendall CW, et al. Colonic health: fermentation and short chain fatty acids. J Clin Gastroenterol 2006;40:235-43.

27. Ritz P, Cloarec D, Beylot M, et al. Effects of colonic fermentation on respiratory gas exchanges following a glucose load in man. Metabolism 1993;42:347-352.

28. Lifschitz CH, Irving CS, Helge H, et al. [13C] Acetate Oxidation in Infants After Oral Versus Rectal Administration: A Kinetic Model. Journal of pediatric gastroenterology and nutrition 1985;4:699-706.

29. Kondo T, Kishi M, Fushimi T, et al. Acetic acid upregulates the expression of genes for fatty acid oxidation enzymes in liver to suppress body fat accumulation. Journal of agricultural and food chemistry 2009;57:5982-6.

30. Yamashita $H$, Maruta $H$, Jozuka $M$, et al. Effects of acetate on lipid metabolism in muscles and adipose tissues of type 2 diabetic Otsuka Long-Evans Tokushima Fatty (OLETF) rats. Bioscience, biotechnology, and biochemistry 2009;73:570-6.

31. Hardie DG, Ross FA, Hawley SA. AMPK: a nutrient and energy sensor that maintains energy homeostasis. Nature reviews Molecular cell biology 2012;13:251-262.

32. van den Hoek AM, Heijboer AC, Voshol PJ, et al. Chronic PYY3-36 treatment promotes fat oxidation and ameliorates insulin resistance in C57BL6 mice. Am J Physiol Endocrinol Metab 2007;292:E238-45.

33. Sloth B, Holst JJ, Flint A, et al. Effects of PYY1-36 and PYY3-36 on appetite, energy intake, energy expenditure, glucose and fat metabolism in obese and lean subjects. American Journal of Physiology-Endocrinology and Metabolism 2007;292:E1062-E1068.

34. Bird AR, Croom WJ, Jr., Fan YK, et al. Peptide regulation of intestinal glucose absorption. J Anim Sci 1996;74:2523-40.

35. Dandona P, Weinstock R, Thusu K, et al. Tumor necrosis factor-alpha in sera of obese patients: fall with weight loss. J Clin Endocrinol Metab 1998;83:2907-10.

36. Tedelind S, Westberg F, Kjerrulf M, et al. Anti-inflammatory properties of the short-chain fatty acids acetate and propionate: a study with relevance to inflammatory bowel disease. World J Gastroenterol 2007;13:2826-32. 
37. Liu T, Li J, Liu Y, et al. Short-chain fatty acids suppress lipopolysaccharide-induced production of nitric oxide and proinflammatory cytokines through inhibition of NF-KB pathway in RAW264. 7 cells. Inflammation 2012;35:1676-1684.

38. Wolever $T$, Brighenti F, Royall $D$, et al. Effect of rectal infusion of short chain fatty acids in human subjects. The American journal of gastroenterology 1989;84:1027.

39. Wolever T, Spadafora P, Eshuis H. Interaction between colonic acetate and propionate in humans. The American journal of clinical nutrition 1991;53:681.

40. Malago JJ, Sangu CL. Intraperitoneal administration of butyrate prevents the severity of acetic acid colitis in rats. Journal of Zhejiang University. Science. B 2015;16:224-34.

41. Child MW, Kennedy A, Walker AW, et al. Studies on the effect of system retention time on bacterial populations colonizing a three-stage continuous culture model of the human large gut using FISH techniques. FEMS Microbiol Ecol 2006;55:299-310.

42. van der Beek CM, Bloemen JG, van den Broek MA, et al. Hepatic Uptake of Rectally Administered Butyrate Prevents an Increase in Systemic Butyrate Concentrations in Humans. J Nutr 2015.

43. Blaak EE, Hul G, Verdich C, et al. Fat oxidation before and after a high fat load in the obese insulin-resistant state. J Clin Endocrinol Metab 2006;91:1462-9. 


\section{SUPPLEMENTAL METHODS}

\section{Calculation of the percentage of increase in fat oxidation due to the oxidation of sodium acetate}

Whole-body fat oxidation rates were calculated according to Frayn et al. ${ }^{1}$, which is based on the $\mathrm{VO}_{2}$ and $\mathrm{VCO}_{2}$ values determined via the open-circuit ventilated hood system. We measured fat oxidation after the administration of the sodium acetate or placebo infusions for $2 \mathrm{~h}$. When we correct the determined $\mathrm{VO}_{2}$ and $\mathrm{VCO}_{2}$ values after the 180 $\mathrm{mmol} / \mathrm{L}$ sodium acetate infusion for the oxidation of 100\% infused sodium acetate $(21.6$ $\mathrm{mmol} \mathrm{NaAc}$ in the $120 \mathrm{~mL}$ infused), this explains $\sim 28 \%$ of the $2 \mathrm{~h}$ increase in fasting fat oxidation (180 mmol/L acetate vs placebo). This is based on the calculations by Akanij et al. ${ }^{2}$. They assumed that the oxidation of $1 \mathrm{~mol}$ of sodium acetate consumes $2 \mathrm{~mol}$ of $\mathrm{O}_{2}$ $(44.8 \mathrm{~L})$ and expires $1 \mathrm{~mol}$ of $\mathrm{CO}_{2}(22.4 \mathrm{~L})$, in our case that would mean:

$21.6 \mathrm{mmol} \mathrm{NaAC}+967.7 \mathrm{mLO}_{2} \rightarrow 483.8 \mathrm{mLCO}_{2}+21.6 \mathrm{mmol} \mathrm{NaHCO}_{3}+19 \mathrm{~kJ}$

The $\mathrm{NaHCO}_{3}$ produced is either increasing the bicarbonate pool size or is excreted in the urine, which causes an extrapulmonary loss of carbon dioxide. These calculated $\mathrm{VO}_{2}$ and $\mathrm{VCO}_{2}$ values for sodium acetate were then subtracted from the total $\mathrm{VO}_{2}$ and $\mathrm{VCO}_{2}$ during the $2 \mathrm{~h}$ period to calculate the nonacetate $\mathrm{VO}_{2}$ and $\mathrm{VCO}_{2}$. The nonacetate values were then used to calculate the fasting fat oxidation. The differences found account for $28.3 \%$ of increased fat oxidation during $2 \mathrm{~h}$.

\section{REFERENCES TO SUPPLEMENTAL METHODS}

1. Frayn K. Calculation of substrate oxidation rates in vivo from gaseous exchange. Journal of Applied Physiology 1983;55:628.

2. Akanji A, Bruce M, Frayn K. Effect of acetate infusion on energy expenditure and substrate oxidation rates in non-diabetic and diabetic subjects. European journal of clinical nutrition. 1989;43(2):107-15. 


\section{SUPPLEMENTAL FIGURES}

Supplemental Figure 2.1. Plasma butyrate and propionate
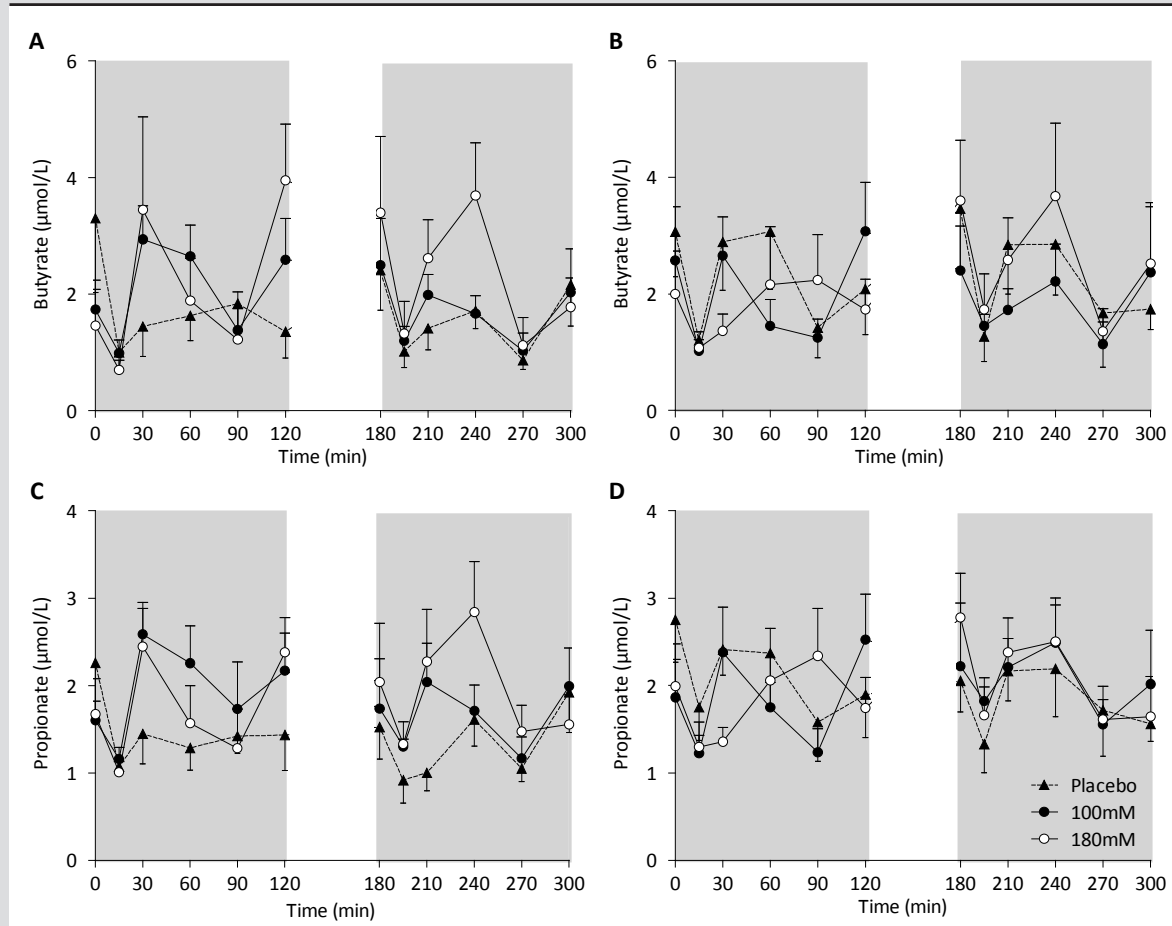

Fasting (t0 - t120) and postprandial (t180 - t300) plasma butyrate after distal (A) and proximal (B) colonic acetate infusions. Fasting (t0 - t120) and postprandial (t180 - t300) plasma propionate after distal (C) and proximal (D) colonic acetate infusions. Values are means \pm SEMs $(n=6)$. No significant differences between interventions. 
Supplemental Figure 2.2. Plasma inflammatory markers
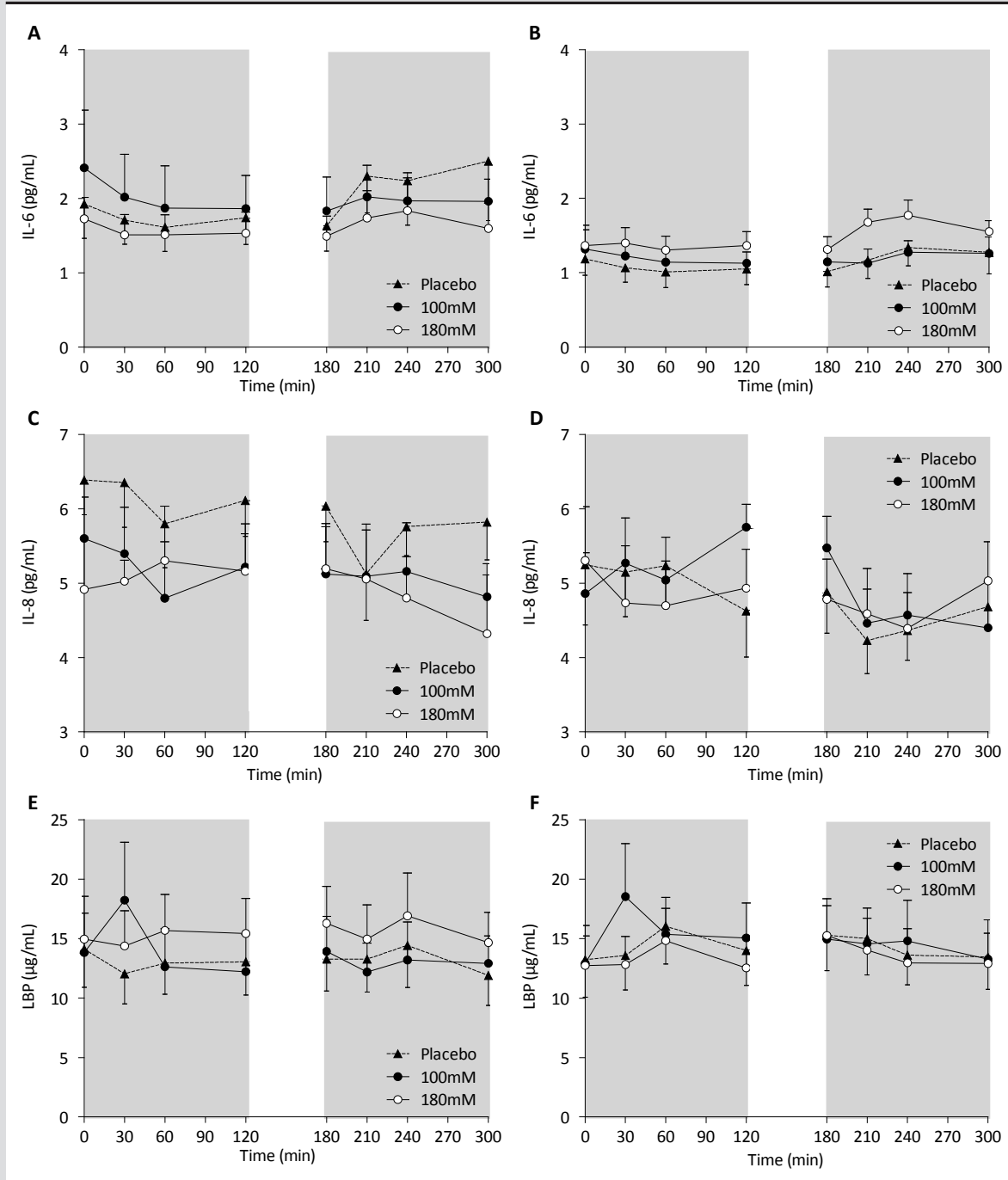

Fasting (t0 - t120) and postprandial (t180 - t300) plasma IL-6 after distal (A) and proximal (B) colonic acetate infusions. Fasting (t0 - t120) and postprandial (t180 - t300) plasma IL-8 after distal (C) and proximal (D) colonic acetate infusions. Fasting (t0 - t120) and postprandial (t180 - t300) plasma LBP after distal (E) and proximal (F) colonic acetate infusions. Values are means \pm SEMs ( $n=6)$. No significant differences between interventions. Abbreviations: IL, interleukin; LBP, lipopolysaccharide binding protein. 
Supplemental Figure 2.3. Plasma lipids
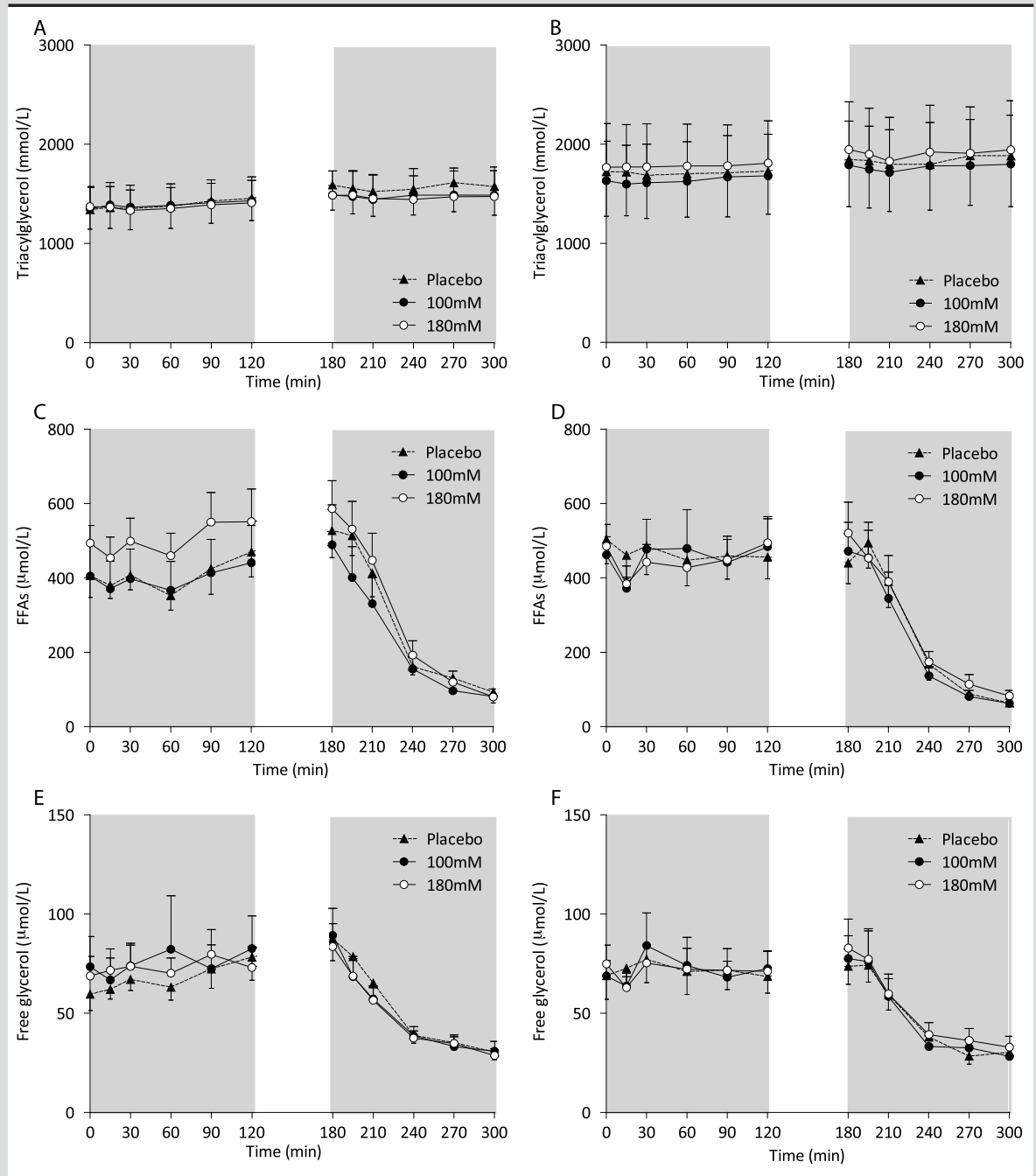

Fasting (t0 - t120) and postprandial (t180 - t300) plasma TAG after distal (A) and proximal (B) colonic acetate infusions. Fasting (t0 - t120) and postprandial (t180 - t300) plasma FFAs after distal (C) and proximal (D) colonic acetate infusions. Fasting (t0 - t120) and postprandial (t180 - t300) plasma free glycerol after distal (E) and proximal (F) colonic acetate infusions. Values are means \pm SEMs $(n=6)$. No significant differences between interventions. Abbreviations: FFA, free fatty acids. 
Supplemental Figure 2.4. Plasma leptin and ANGPTL4
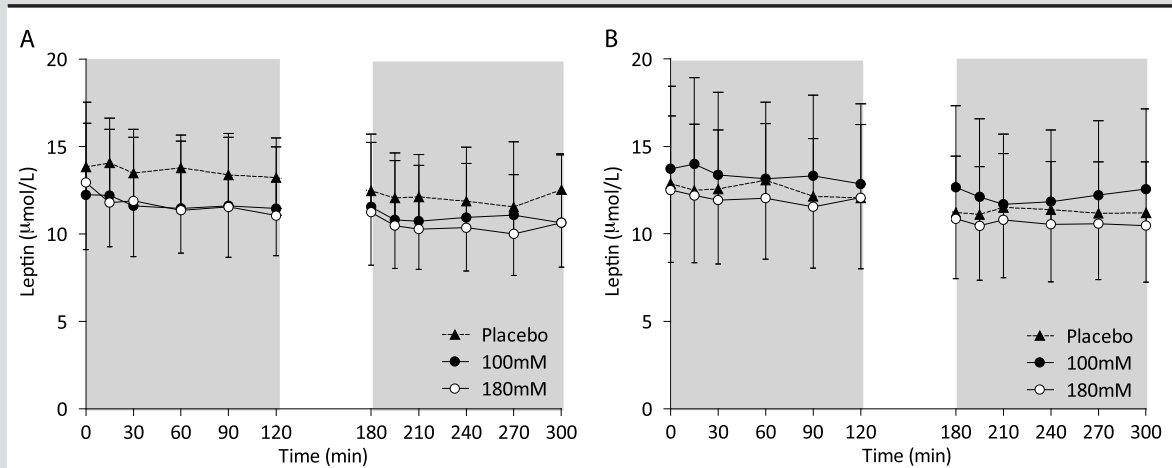

C
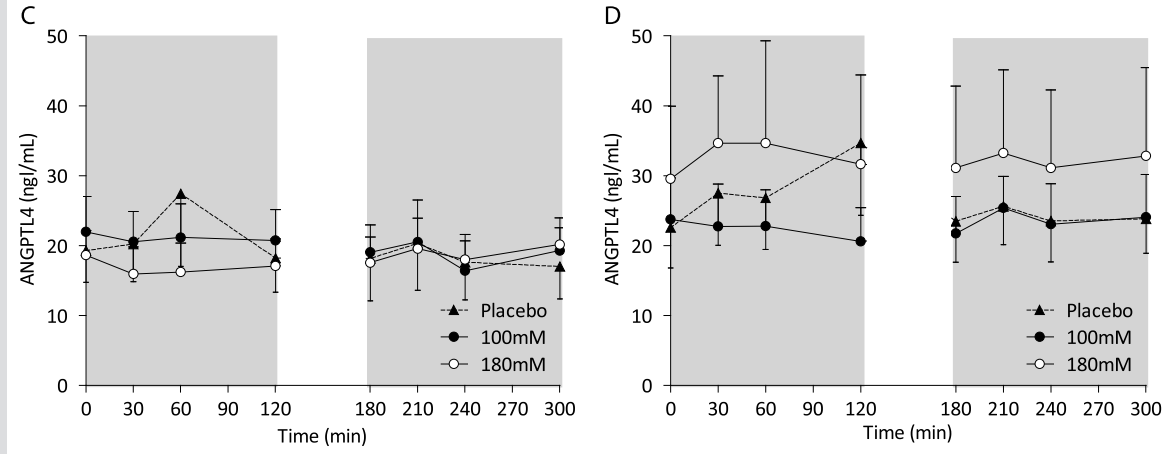

Fasting (t0 - t120) and postprandial (t180 - t300) plasma leptin after distal (A) and proximal (B) colonic acetate infusions. Fasting (t0 - t120) and postprandial (t180 - t300) plasma ANGPTL4 after distal (C) and proximal (D) colonic acetate infusions. Values are means \pm SEMs $(n=6)$. No significant differences between interventions. Abbreviations: ANGPTL4, angiopoietin-like protein 4. 



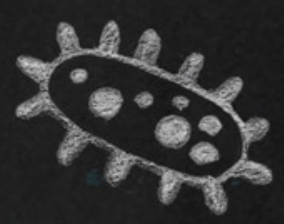

\section{CHAPTER 3}

\section{The prebiotic inulin improves substrate metabolism and promotes short-chain fatty acid production in overweight to obese men}

Christina M. van der Beek, Emanuel E. Canfora, Anna M. Kip, Stefan H. M. Gorissen, Steven W. M. Olde Damink, Hans M. van Eijk, Jens J. Holst, Ellen E. Blaak, Cornelis H. C. Dejong, Kaatje Lenaerts 


\section{ABSTRACT}

Background: Human gut microbiota play an important role in maintaining human health. Dietary fibers, i.e. prebiotics, escape digestion and are fermented by human gut microbiota into the short-chain fatty acids (SCFAs) acetate, propionate, and butyrate. SCFAs promote fat oxidation and improve metabolic health. Therefore, the prebiotic inulin might be an effective dietary strategy to beneficially affect human metabolism.

Objective: We aimed to investigate acute metabolic effects of inulin compared with digestible carbohydrates and to trace inulin-derived SCFAs using stable isotope tracer methodology.

Methods: In a double-blind, randomized, placebo-controlled crossover design, 14 healthy, overweight men consumed a high-fat milkshake containing A) $24 \mathrm{~g}$ inulin of which $0.5 \mathrm{~g}$ was $U_{-13}{ }^{13}$-inulin (INU) or B) 24 g maltodextrin (placebo, PLA), with a wash-out period of at least five days. Fat oxidation was measured via an open-circuit ventilated hood and blood samples were collected up to $7 \mathrm{~h}$ after ingestion. Breath samples for ${ }^{13} \mathrm{CO}_{2}$ measurements, hunger and satiety scores, and fecal samples for SCFA analyses were collected.

Results: Fat oxidation increased in the early postprandial phase $(0$ - $3 \mathrm{~h})$, and both plasma glucose and insulin were lower after INU ingestion compared with PLA (all $P<0.05$ ). Plasma free fatty acids were higher in the early, and lower in the late postprandial period after INU ingestion. Inulin was fermented into SCFAs as indicated by higher plasma acetate concentrations after INU intake than PLA $(P<0.05)$. In addition, we found continuous increases in plasma ${ }^{13} \mathrm{C}$-SCFA enrichments ( $P<0.05$ from $\mathrm{t}=120$ onwards) and breath ${ }^{13} \mathrm{CO}_{2}$ enrichments after INU intake. There were no effects on plasma triacylglycerol, free glycerol, satiety hormones GLP-1 and PYY, and hunger and satiety scores.

Conclusions: Ingestion of the prebiotic inulin improves fat oxidation and promotes SCFA production in overweight to obese men. Overall, replacing digestible carbohydrates with the fermentable inulin may favor human substrate metabolism. 


\section{INTRODUCTION}

Obesity and the metabolic syndrome are rapidly expanding health threats. ${ }^{1}$ Body weight, insulin sensitivity, and glucose metabolism in humans are found to be related to the gut microbiota. ${ }^{2-4}$ Gut microbial composition and activity can be influenced by ingesting dietary fibers, also called prebiotics. A prebiotic is defined as a non-digestible food ingredient that beneficially affects the host by selectively stimulating the growth and/or activity of one or a limited number of bacterial species in the colon. ${ }^{5}$ One important activity of the human gut microbiota is fermentation of dietary fibers into short chain fatty acids (SCFAs), mainly acetate, propionate, and butyrate. Recent evidence suggests that increasing colonic and plasma SCFA concentrations could be used as a strategy to prevent and treat obesity and obesity-induced insulin resistance..$^{3,6,7}$ In addition, indigestible prebiotics have a lower blood glucose-raising potential, i.e. lower glycemic index, than digestible carbohydrates, thereby promoting glucose homeostasis. ${ }^{8}$ On the contrary, a recent study in rats showed that continuous intragastric acetate infusions decreased insulin sensitivity. ${ }^{9}$ Therefore, human studies investigating metabolic effects concerning prebiotics and consequential SCFA formation are warranted.

Inulin is a prebiotic that targets gut microbiota and thereby influences microbial composition and activity. Studies applying in vitro models have indicated that the human gut microbiota can ferment inulin into acetate, propionate, and butyrate in considerable amounts. ${ }^{10-13}$ In addition, several human studies have shown that daily inulin supplementation increases fecal bifidobacteria, indicating an alteration in gut microbial composition. ${ }^{14,15}$ A longer-term inulin supplementation caused reductions in body weight, BMI, as well as energy and fat intake in patients with type 2 diabetes. ${ }^{16}$ In addition, replacing digestible carbohydrates with inulin for three to eight weeks in healthy subjects reduced plasma lipid concentrations, mostly triglycerides, ${ }^{15,17-20}$ but also LDL-cholesterol and total cholesterol, ${ }^{21}$ and lowered fasting glucose and insulin concentrations. ${ }^{16,20,22}$

Only few human studies have investigated underlying mechanisms contributing to the positive effects of inulin on human metabolism. Importantly, $24 \mathrm{~g}$ of oral inulin intake increased serum concentrations of acetate, propionate, and butyrate 4 to 6 hours after intake, supporting the hypothesis that fermentation into SCFAs may lead to metabolic effects after inulin ingestion. ${ }^{23}$ In addition, a recent study from our research group has shown that colonic infusion of $22 \mathrm{mmol}$ of the SCFA acetate in overweight to obese males caused an acute increase in fasting fat oxidation and peptide YY (PYY), a satiety hormone reducing appetite. ${ }^{7}$ The exact mechanism by which inulin influences host' substrate metabolism and whether inulin-derived SCFAs contribute to the observed metabolic effects in humans remains to be investigated. 
This study was designed to compare the acute effects of a mixed high-fat liquid meal containing digestible carbohydrates with a mixed meal where a considerable amount of the digestible carbohydrates was replaced by the fermentable carbohydrate inulin. We hypothesized that dietary inulin is fermented by the gut microbiota into SCFAs in the late (3 - 7 h) postprandial period..$^{23}$ We recently showed that colonically administered acetate increases human fat oxidation. ${ }^{7}$ The primary aim of the current study was to investigate the early and late postprandial effects of oral inulin intake on fat oxidation and energy expenditure. Secondary outcomes include the effect of inulin on carbohydrate oxidation, circulating metabolites (glucose, triacylglycerol (TAG), FFAs, free glycerol), plasma hormones (insulin, GLP-1, PYY), plasma and fecal SCFA concentrations and enrichments, as well as appetite. The application of a unique stable isotope technique allowed us to assess whether inulin is fermented into SCFAs and to explore the kinetics of SCFA production and availability in humans. 


\section{SUBJECTS AND METHODS}

\section{Study population}

Fifteen healthy, normoglycemic, Caucasian men aged 20 - 50 y with a body mass index between 25 and $35 \mathrm{~kg} / \mathrm{m}^{2}$ were recruited between January 2014 and July 2014 from the vicinity of Maastricht, The Netherlands. Subjects were excluded for any of the following reasons: having a fasting plasma glucose $>7.0 \mathrm{mmol} / \mathrm{L}$, having known gastroenterological diseases or major abdominal surgery in the past, having cardiovascular diseases, cancer, liver, or kidney malfunction, patients with a life expectancy of less than $5 \mathrm{y}$, alcohol or drug abuse, excessive smoking (>20 cigarettes per day), on a weight loss intervention, use of prebiotics, probiotics, or antibiotics during or 3 months prior to the study, vigorous exercise training, use of any medication that influences glucose or fat metabolism and inflammation, and regular use of laxatives. All procedures were approved by the Medical Ethics Committee of Maastricht University Medical Center+ (MUMC+) and conducted in accordance with the Declaration of Helsinki (revised version, October 2008, Seoul, South Korea). Written informed consent was obtained from all subjects. This clinical study was monitored by clinical research associates of the Clinical Trial Center Maastricht (CTCM, Maastricht, The Netherlands).

\section{Study design}

The study was a double-blind, randomized, placebo-controlled crossover trial. An independent researcher performed randomization and gave the randomization key to the principle investigator at the completion of the study. Subjects were investigated during two investigation days with at least 5 days of washout period in between. Previous trials have shown that this period is long enough to make sure that no carry-over effect is present. ${ }^{7}$ At the start of each investigation day, the subjects consumed a high-fat milkshake containing: a) $24 \mathrm{~g}$ inulin (Sensus, Roosendaal, The Netherlands, average degree of polymerization (DP 8) of which 0.5 g was $U-{ }^{13} \mathrm{C}$-labeled inulin (DP 3 - 60, IsoLife, Wageningen, The Netherlands), referred to as INU and providing $\sim 0.21 \mathrm{MJ}$, or b) $24 \mathrm{~g}$ maltodextrin (Avebe, Veendam, The Netherlands) as placebo (PLA), providing $\sim 0.42 \mathrm{MJ}$. The milkshake provided $2.3 \mathrm{MJ}$ energy and consisted of 46 En\% fat (28 g), 42 En\% carbohydrates (58 g), and 12 En\% protein (17 g). 
Figure 3.1. Protocol of the investigation day

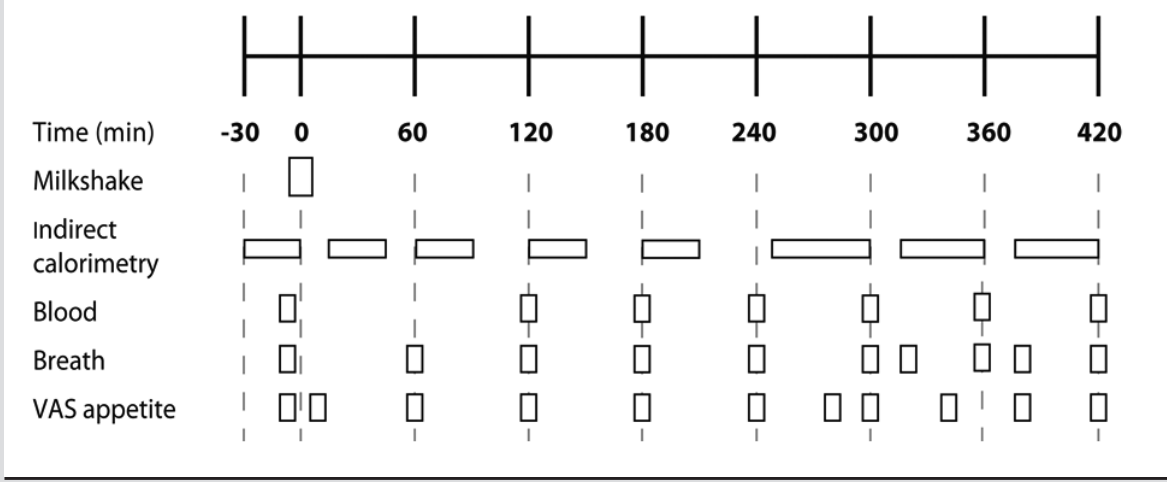

Abbreviations: VAS: Visual Analogue Scale.

Figure 3.1 shows the design of the investigation day. On the evening before the investigation day, subjects were provided with a standardized meal composed of 65 En\% carbohydrates, 20 En\% fat, 15 En\% protein. Furthermore, subjects were asked to refrain from ingesting naturally ${ }^{13} \mathrm{C}$-enriched food products such as corn, sugar cane, or pineapple. Every investigation day started with baseline measurements of indirect calorimetry, blood and breath sampling, and Visual Analogue Scale (VAS) assessment for hunger and satiety. After baseline measurements, the subjects ingested a high-fat milkshake containing INU or PLA. Thereafter, on various time points for $7 \mathrm{~h}$, multiple blood and breath samples were collected, indirect calorimetry was performed, and VAS for hunger and satiety were obtained. In addition, the subjects were asked to sample stool the first day after each investigation day.

\section{Calculations}

The primary outcome parameter, fat oxidation, was measured using an open-circuit ventilated hood system (Omnical, MUMC+, The Netherlands). $\mathrm{CO}_{2}$ production $\left(\mathrm{VCO}_{2}\right.$ in $\mathrm{L} / \mathrm{min}$ ) and $\mathrm{O}_{2}$ consumption $\left(\mathrm{VO}_{2}\right.$ in $\left.\mathrm{L} / \mathrm{min}\right)$ were measured during eight time periods: $\mathrm{t}=-30$ - 0 , 15 - 40, 60 - 85, 120 - 145, 180 - 205, 250 - 300, 320 - 360, and 380 - 420 min (Figure 3.1). The equations of Weir ${ }^{24}$ and Frayn ${ }^{25}$ were used to calculate resting energy expenditure and the total rate of fat and carbohydrate oxidation. The estimated nitrogen ( $N$ ) excretion was calculated based on the assumption that protein oxidation represents $15 \%$ of total energy expenditure. 


\section{Analyses}

Blood samples were collected from an antecubital vein at 7 different time points on each investigation day (Figure 3.1). Blood was collected into pre-chilled 0.2 M EDTA tubes (Sigma Dorset, UK) for analysis of FFAs, TAG, free glycerol, glucose, insulin, and SCFAs. For GLP-1 analysis, blood was collected in a $2 \mathrm{~mL}$ EDTA tube in which $20 \mu \mathrm{L}$ of dipeptidyl peptidase (DPP)-4 inhibitor was added. For PYY analysis, blood was collected in $2 \mathrm{~mL}$ aprotinin-EDTA tubes in which $20 \mu \mathrm{L}$ of DPP-4 inhibitor was added. The samples were centrifuged at 3,500 $\mathrm{g}$ at $4^{\circ} \mathrm{C}$ for $10 \mathrm{~min}$. Plasma was aliquoted, directly snap-frozen in liquid nitrogen, and stored at $-80^{\circ} \mathrm{C}$ until analysis.

Plasma FFAs, TAG, and glucose were measured with enzymatic assays on an automated spectrophotometer (ABX Pentra 400 autoanalyzer, Horiba ABX, Montpellier, France). Plasma free glycerol was measured after precipitation, using an enzymatic assay (EnzytecTM Glycerol, Roche Biopharm, Switzerland) on a Cobas Fara spectrophotometric auto-analyzer (Roche Diagnostics, Basel, Switzerland). The concentrations of insulin and PYY were determined with commercially available radioimmunoassay (RIA) kits (Human Insulin specific RIA and Human PYY (3-36) RIA, Millipore Corporation, MA, USA). Plasma samples were assayed for total GLP-1 immunoreactivity using an antiserum, which reacts equally with intact GLP-1 and the primary (N-terminally truncated) metabolite as previously described. ${ }^{26}$ Deproteinization and subsequent preparation of plasma samples for analysis of SCFA concentrations was performed as reported before. ${ }^{27}$ Briefly, plasma samples were deproteinized using acidified methanol and centrifuged at 50,000 g for $10 \mathrm{~min}$ at $4^{\circ} \mathrm{C}$. The clear supernatant was transferred to a $300 \mu \mathrm{L}$ glass micro-insert, spring loaded in a $4 \mathrm{~mL}$ vial, equipped with a self-sealing septum. Analysis was performed using liquid chromatography-mass spectrometry. SCFA concentrations of acetate, butyrate, and propionate were determined using calibration curves of standards with known SCFA concentrations. The detection limits for acetate, propionate, and butyrate of this method were 0.1, 0.05, and $0.05 \mu \mathrm{mol} / \mathrm{L}$, respectively. Plasma ${ }^{13} \mathrm{C}$-labeled SCFA were analyzed as described before. ${ }^{28}$ Plasma samples were deproteinized and extracted according to Morrison et al. ${ }^{29}$. The extracted samples for analysis of ${ }^{13} \mathrm{C}$ enrichment of SCFAs were injected $(4 \mu \mathrm{L})$ in splitless mode at $240{ }^{\circ} \mathrm{C}$ into a Delta XP isotope ratio mass spectrometer (Thermo Fisher Scientific, Bremen, Germany) equipped with a Trace gas chromatograph and a combustion interface type 3 (GC-C-IRMS). An AT Aquawax-DA column (30 m x $0.53 \mathrm{~mm}$ i.d. and $1.00 \mu \mathrm{m}$ film thickness; Grace, Lokeren, Belgium) was used. The initial oven temperature was $80^{\circ} \mathrm{C}$ (3 min) and ramped to $140{ }^{\circ} \mathrm{C}$ at a rate of $4^{\circ} \mathrm{C} / \mathrm{min}$ followed by an increase to $240{ }^{\circ} \mathrm{C}(10$ min) at a rate of $16^{\circ} \mathrm{C} / \mathrm{min}$. Helium was used as carrier gas in a constant flow mode of 2.5 $\mathrm{mL} / \mathrm{min}$. After elution from the GC column, the compounds were oxidized into $\mathrm{CO}_{2}$ in the combustion reactor after which the enrichment was measured in the IRMS detector. To calculate the enrichment of the SCFAs from the measured ${ }^{13} \mathrm{CO}_{2}$ enrichment, the number of labeled carbon atoms per molecule and the enrichment of the administered substrate 
( ${ }^{13} \mathrm{C}$-SCFAs) were taken into account. Data were processed using Isodat NT (version 2.0, Finnigan ${ }^{\mathrm{T}}$, Thermo Fisher Scientific).

Breath samples were collected via a mixing chamber in $12 \mathrm{~mL}$ exetainers at 10 different time points (Figure 3.1). These were analyzed for ${ }^{13} \mathrm{CO}_{2}$ using gas chromatography isotope ratio mass spectrometry (GC-IRMS). Scores for hunger and satiety were measured using VAS from 0 to $100 \mathrm{~mm}$. VAS scores were collected at 11 different time points (Figure 3.1). Volunteers were asked to indicate which position on a line best reflected their feeling concerning hunger, full feeling, satiety, and desire to eat.

The day after every investigation day, the subjects collected a fecal sample from their first stool. The samples were directly stored on dry ice at home. Upon arrival in the laboratory, fecal samples were weighed and frozen at $-80{ }^{\circ} \mathrm{C}$. Fecal acetate, propionate, and butyrate were measured by gas chromatography-mass spectrometry (GC-MS, Medical laboratory 'Dr. Stein \& Collegae', Mönchengladbach, Germany), according to the method described by Garciá-Villalba et al. ${ }^{30}$. Suspensions of fecal samples (100 mg in $1 \mathrm{~mL} 4 \mathrm{M}$ $\mathrm{NaCl}$ ) for ${ }^{13} \mathrm{C}$-labeled SCFAs were acidified with $\mathrm{H}_{2} \mathrm{SO}_{4}(150 \mu \mathrm{L})$ and extracted into diethyl ether ( $3 \mathrm{~mL}$ ). The ether layer was dried over $\mathrm{Na}_{2} \mathrm{SO}_{4}$ and injected into the GC-C-IRMS. Prepared fecal samples were analyzed as described above in the section on plasma ${ }^{13} \mathrm{C}$ labeled SCFAs.

\section{Statistical analyses}

Values are expressed as mean \pm SEM. For energy expenditure, substrate oxidation, plasma metabolites, and VAS scores, differences between INU and PLA were tested using twoway repeated measures analysis of variance (rANOVA) for time and treatment. In case of a significant time $x$ treatment interaction, a post-hoc test with Bonferroni correction was performed to compare treatment effects at specific time points. Differences in incremental area under the curve (iAUC) over the early ( 0 - 3 h) and late (3 - 7 h) postprandial period after treatment were compared using a paired t-test. Relative changes in energy expenditure and substrate oxidation compared to baseline are reported as iAUC. For ${ }^{13} \mathrm{CO}_{2}$ and ${ }^{13} \mathrm{C}$-SCFA enrichments, rANOVA for time with post-hoc test with Bonferroni correction, and pairwise comparisons at specific time points vs baseline are reported. A Pearson's correlation coefficient was computed to assess the relationship between the primary outcome, fat oxidation, and SCFAs. For calculation of correlations, we included all time points at which we measured both fat oxidation and plasma SCFAs. Statistical analysis was performed using SPSS 22.0 and a two-sided $\mathrm{P}<0.05$ was considered statistically significant. The sample-size calculation, with a 2-sided significance level of 0.05 and power of 0.8, was based on a study by Higgins et $a{ }^{3}{ }^{31}$, showing an increase in postprandial fat oxidation of $23 \%$ after intake of a meal rich in resistant starch compared with placebo. The sample size was estimated at $n=15$ including an expected dropout rate of 10\%. 


\section{RESULTS}

\section{Subject characteristics}

Fifteen healthy subjects were included in the study. One subject discontinued intervention due to having a migraine attack during one of the investigation days and therefore being unable to complete the test period (Supplemental Figure 3.1). None of the subjects reported any side-effects. Table 1 shows the baseline characteristics of the subjects.

Table 3.1. Baseline values of male participants $(n=14)$

\begin{tabular}{lr}
\hline Variable & Mean \pm SEM \\
\hline Age $(\mathrm{y})$ & $34.1 \pm 2.8$ \\
$\mathrm{BMI}\left(\mathrm{kg} / \mathrm{m}^{2}\right)$ & $30.4 \pm 0.7$ \\
Waist-to-hip ratio & $1.0 \pm 0.01$ \\
Fasting glucose (mmol/L) & $5.2 \pm 0.02$ \\
Hemoglobin (mmol/L) & $9.5 \pm 0.02$ \\
ALT (U/L) & $36.1 \pm 4.9$ \\
HbA ${ }_{1 C}(\%)$ & $5.2 \pm 0.2$ \\
Creatinine (mmol/L) & $93.4 \pm 3.4$ \\
\hline
\end{tabular}

Reported metabolites are measured in plasma.

Abbreviations: ALT, Alanine aminotransferase; HbA1C, Hemoglobin $\mathrm{A}_{1 \mathrm{C}}$.

\section{Fat oxidation, carbohydrate oxidation, and energy expenditure}

Baseline fasting fat oxidation was $0.056 \pm 0.001 \mathrm{~g} / \mathrm{min}$ and $0.070 \pm 0.002 \mathrm{~g} / \mathrm{min}$ for INU and PLA resp. ( $P=0.025)$. Fat oxidation increased over time to a greater extent after INU treatment when compared with PLA ( $P=0.003)$. Early postprandial fat oxidation was higher after INU intake when compared with PLA (iAUC 0 - 3 h: $0.85 \pm 0.12$ vs $-0.88 \pm 0.12 \mathrm{~g}$ fat 3 $h^{-1}, P=0.011$, Figure 3.2A and 3.2B). Fat oxidation was not significantly different between INU and PLA intake in the late postprandial phase.

Immediately after milkshake ingestion, carbohydrate oxidation increased with a highest concentration at $\mathrm{t}=60 \mathrm{~min}$ in both conditions. INU intake resulted in a lower in carbohydrate oxidation when compared with PLA at $\mathrm{t}=60$ min $(P=0.030)$. Early postprandial carbohydrate oxidation was lower in the INU group when compared with PLA (iAUC 0 - 3 h: $3.47 \pm 0.35$ vs $8.28 \pm 0.35 \mathrm{~g} \mathrm{CHO} 3 \mathrm{~h}^{-1}$, Figure 3.2C and 3.2D). Carbohydrate oxidation was not significantly different between INU and PLA intake in the late postprandial phase. 
Figure 3.2. Fat oxidation, carbohydrate oxidation, and energy expenditure

A

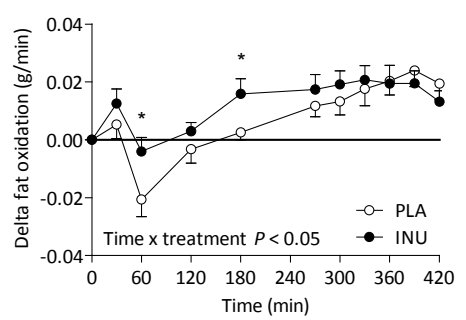

C
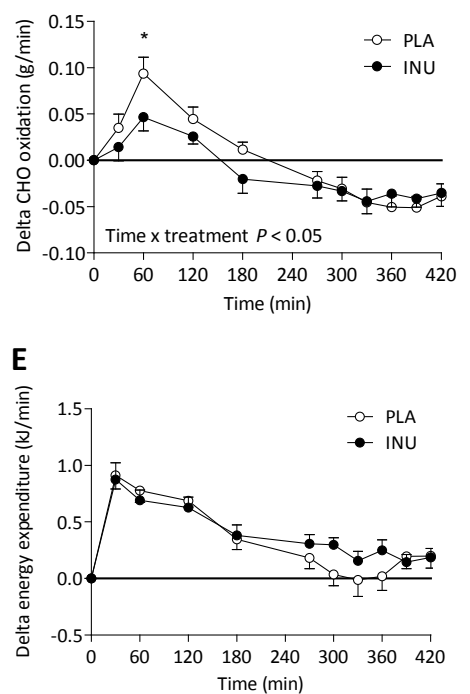

B

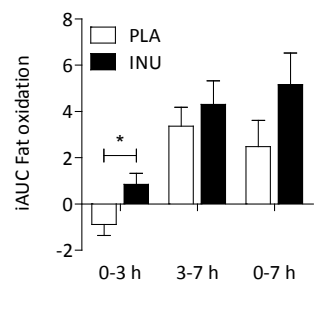

D

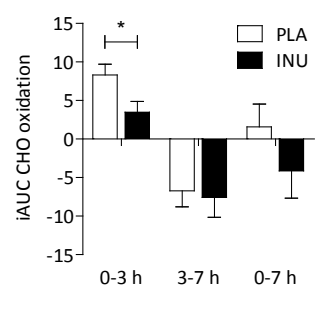

$\mathbf{F}$

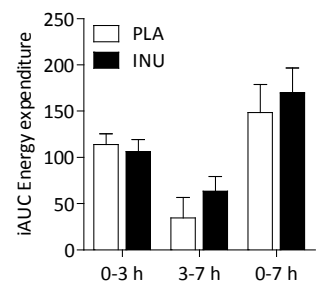

Relative change in mean ( \pm SEM) fat oxidation ( $\mathbf{A}$ and $\mathbf{B})$, carbohydrate oxidation (C and $\mathbf{D}$ ), and energy expenditure ( $\mathbf{E}$ and $\mathbf{F}$ ), and corresponding incremental area under the curve (iAUC) after consumption of inulin or placebo $(n=14)$ in the early and late postprandial phase. Data were analyzed with a 2-factor repeated-measures ANOVA. Data for iAUC were analyzed using a paired t-test. *Different from placebo, $P<0.05$. Only significant changes $(P<0.05)$ or trends $(P<0.1)$ on time $x$ treatment or treatment are reported. Abbreviations: $\mathrm{CHO}$, carbohydrate oxidation; INU, Inulin; PLA, Placebo. 
Energy expenditure increased immediately after intake of both the INU and PLA treatment, and tended to be higher after the INU intake when compared with placebo over the whole test period of $7 \mathrm{~h}(P=0.059)$, but did not significantly differ in the early nor late postprandial phase (Figure 3.2E and 3.2F).

\section{Plasma lipids}

Baseline FFA plasma concentrations before ingestion of the milkshake differed significantly between the two interventions ( $P=0.030$ at $\mathrm{t}=0$ ). Immediately after intake of the milkshake, plasma free fatty acid concentrations fell in both treatments, with a lowest concentration reached at $120 \mathrm{~min}$, and thereafter values increased again. The INU treatment significantly reduced the early postprandial decrease in plasma FFAs (Figure 3.3A, $P<0.01$ ). INU intake therefore resulted in significantly higher plasma FFAs in the early postprandial phase when compared with PLA (iAUC 0 - 3h: -23 $027 \pm 726$ vs $-39615 \pm 1356 \mu \mathrm{mol} / \mathrm{L} \mathrm{FFA} 3 \mathrm{~h}^{-1}, P=0.009$, (Figure 3.3B). FFAs were significantly lower at time point $t=360$ and $t=420$ min after INU when compared with PLA (both $P<0.05$ ). There were no significant differences in plasma free glycerol and TAG between INU and PLA treatment (Figure 3.3C-F).

\section{Plasma glucose, insulin, and satiety hormones}

The high-fat milkshake increased plasma glucose and insulin concentrations directly after intake, independent of the treatment. The increase in plasma glucose was however lower after the INU treatment when compared with PLA ( $5.4 \pm 0.03$ vs $5.8 \pm 0.04 \mathrm{mmol} / \mathrm{L}$ at time point $t=120, P=0.011$, Figure 3.4A). INU caused a lower glucose response in the early postprandial phase when compared with PLA (iAUC 0 - 3 h: $8.12 \pm 3.07$ vs $46.97 \pm 3.57 \mathrm{mmol} / \mathrm{L}$ $3 \mathrm{~h}^{-1}, P=0.002$, Figure 3.4B). In the late postprandial phase, no difference in plasma glucose concentrations was observed between INU and PLA treatment. In line with the findings on plasma glucose, INU caused a lower plasma insulin concentration when compared with PLA ( $P<0.001$, Figure 3.4C). In addition, INU intake caused a lower insulin response in both the early postprandial phase (iAUC 0 - 3h: $1494 \pm 81$ vs $3523 \pm 161 \mathrm{mU} / \mathrm{L} 3 \mathrm{~h}^{-1}, P=0.001$ ), and the late postprandial phase (iAUC 3 - 7h: $-108.4 \pm 43.4$ vs $506.4 \pm 75.0 \mathrm{mU} / \mathrm{L} 4 \mathrm{~h}^{-1}, P=0.002$, Figure 3.4D) when compared with PLA.

There were no significant differences in plasma concentrations of satiety hormones GLP-1 and PYY between INU and PLA treatment, neither in the early nor in the late postprandial phase (Figure 3.4E-H). 
Figure 3.3. Plasma lipids

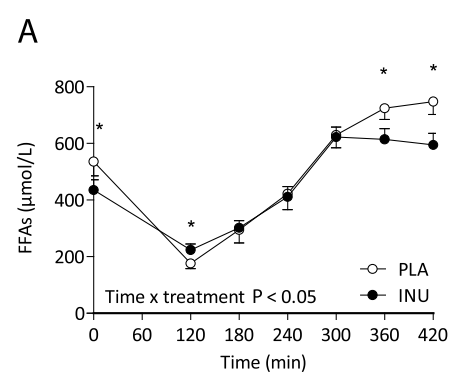

C

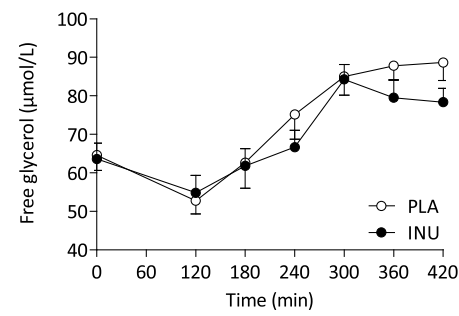

$\mathrm{E}$

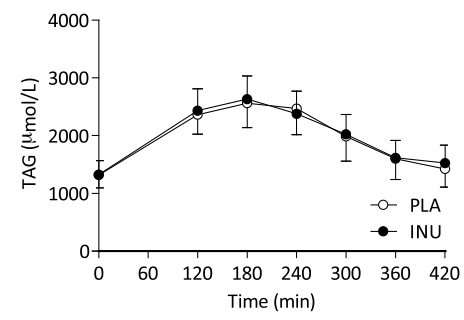

B

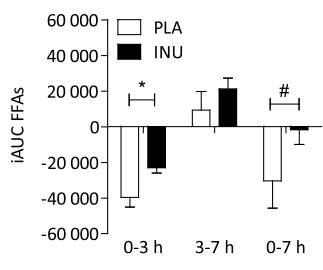

D

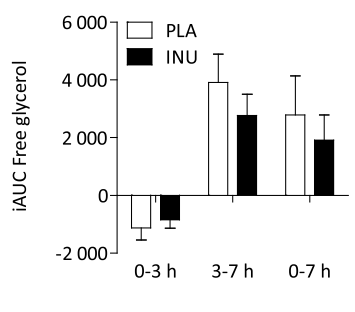

$\mathrm{F}$

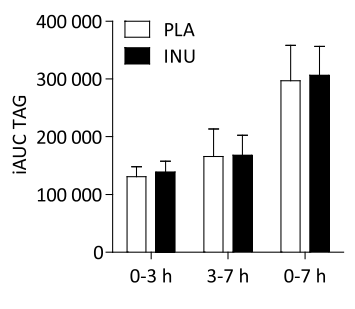

Mean ( \pm SEM) FFAs (A and $\mathbf{B}$ ), free glycerol (C and $\mathbf{D})$, and TAG (E and $\mathbf{F})$, and corresponding incremental area under the curve (iAUC) after consumption of inulin or placebo $(n=14)$ in the early and late postprandial phase. Data were analyzed with a 2 -factor repeated-measures ANOVA. Data for iAUC were analyzed using a paired t-test. *Different from placebo, $P<0.05$. Only significant changes $(P<0.05)$ or trends $(P<0.1)$ on time $x$ treatment or treatment are reported. Abbreviations: FFA, Free fatty acid; INU, Inulin; PLA, Placebo; TAG, triacylglycerol. 
Figure 3.4. Plasma glucose, insulin, and satiety hormones

A

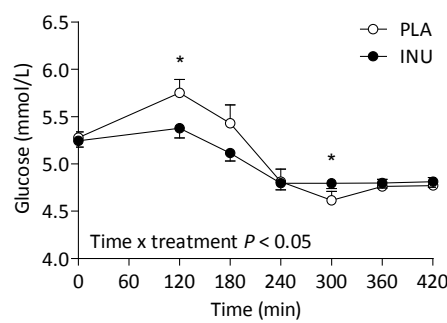

C

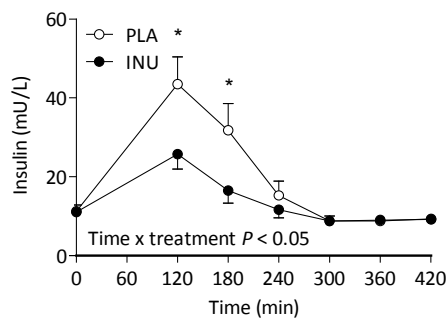

E

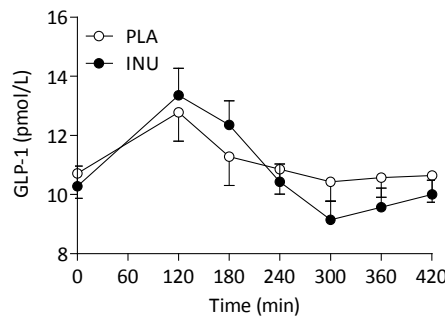

G

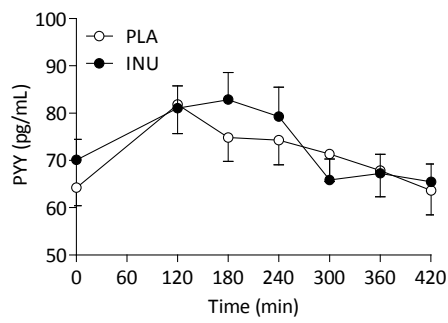

B

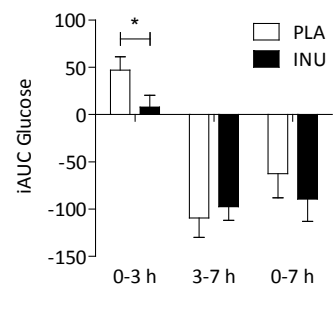

D

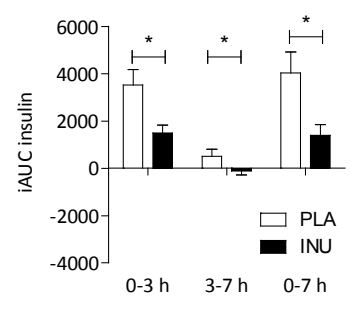

$\mathbf{F}$

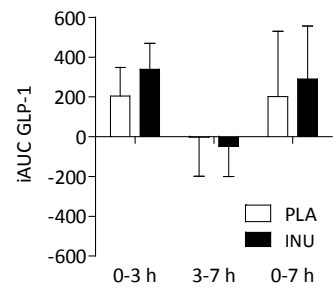

H

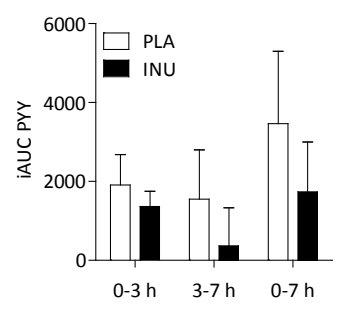

Mean ( \pm SEM) glucose (A and $\mathbf{B})$, insulin (C and $\mathbf{D})$, GLP-1 (E and $\mathbf{F}$ ) and PYY (G and $\mathbf{H})$, and corresponding incremental area under the curve (iAUC) after consumption of inulin or placebo $(n=14)$ in the early and late postprandial phase. Data were analyzed with a 2 -factor repeated-measures ANOVA. Data for iAUC were analyzed using a paired t-test. *Different from placebo, $P<0.05$. Only significant changes $(P<0.05)$ or trends $(P<0.1)$ on time $x$ treatment or treatment are reported. Abbreviations: INU, Inulin; PLA, Placebo. 


\section{Plasma SCFA concentrations and enrichments}

INU intake resulted in higher plasma acetate concentrations when compared with PLA $(P=0.001$, Figure 3.5A). Plasma acetate was higher in the late postprandial phase (iAUC 3 7h: $1330 \pm 358 \mathrm{vs}-7987 \pm 559 \mu \mathrm{mol} / \mathrm{L} 4 \mathrm{~h}^{-1}, P=0.002$, Figure 3.5B), resulting in an increase in total plasma acetate over the $7 \mathrm{~h}$ test period (iAUC 0 - 7h: $1310 \pm 522$ vs -10 $169 \pm 834$ $\mu \mathrm{mol} / \mathrm{L} 7 \mathrm{~h}^{-1}, P=0.007$ ). There was no significant difference in plasma propionate (Figure 3.5C and 3.5D) or plasma butyrate (Figure 3.5E and 3.5F) when compared with PLA. INU treatment, however, resulted in a trend towards higher plasma butyrate concentrations when compared with PLA over the whole test period (Figure 3.5E and 3.5F, $P=0.056$ ). Fat oxidation significantly correlated with plasma propionate $(r=0.250, P=0.008)$ and plasma butyrate $(r=0.231, P=0.014)$, but not with plasma acetate. The correlation coefficient was in both cases, however, so low that it is considered to be negligible. 
Figure 3.5. Plasma SCFA concentrations
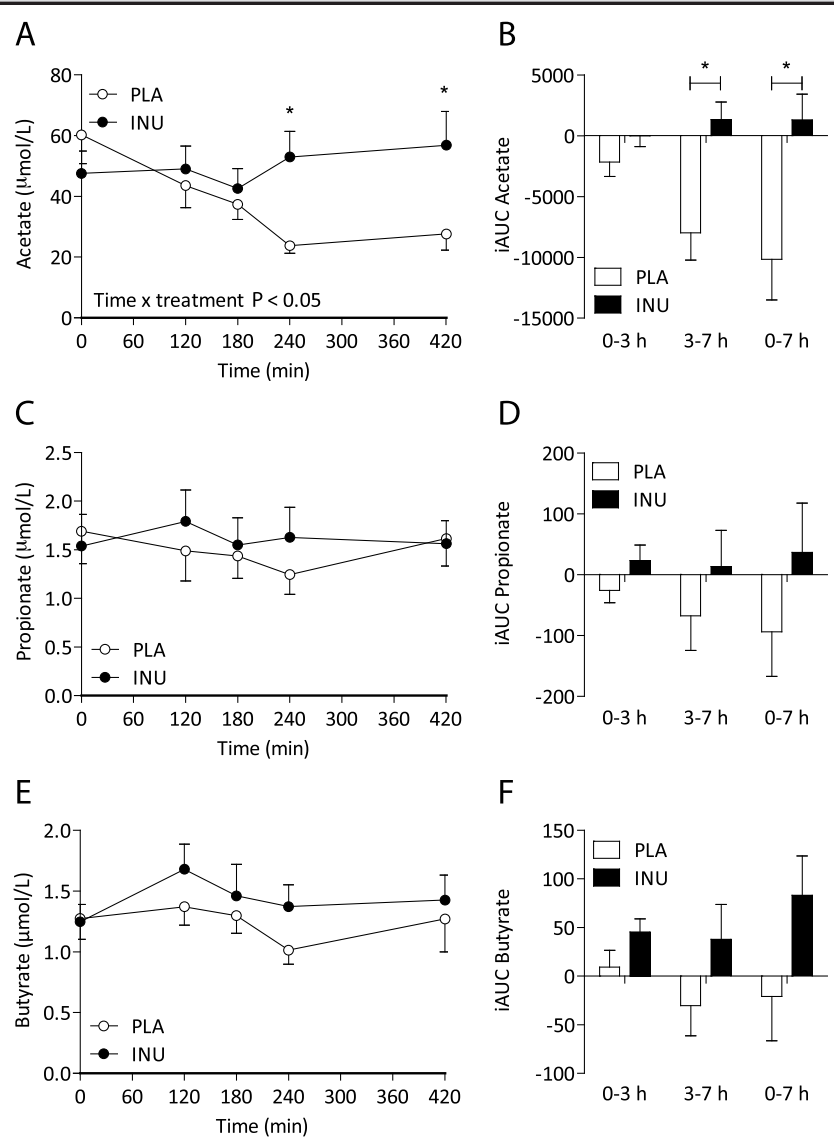

Mean ( \pm SEM) acetate (A and $\mathbf{B}$ ), propionate ( $\mathbf{C}$ and $\mathbf{D}$ ) and butyrate ( $\mathbf{E}$ and $\mathbf{F})$, and corresponding incremental area under the curve (iAUC) after consumption of inulin or placebo $(n=14)$ in the early and late postprandial phase. Data were analyzed with a 2-factor repeated-measures ANOVA. Data for iAUC were analyzed using a paired t-test. *Different from placebo, $P<0.05$. Only significant changes $(P<0.05)$ or trends $(P<0.1)$ on time $x$ treatment or treatment are reported. Abbreviations: INU, Inulin; PLA, Placebo. 
Plasma ${ }^{13} \mathrm{C}$-SCFAs and exhaled ${ }^{13} \mathrm{CO}_{2}$ increased significantly directly after INU intake (Figure 3.6). A pairwise comparison showed a significant effect from time point $t=120$ onwards for ${ }^{13} \mathrm{C}$-SCFAs (Acetate $\mathrm{t}=120 P<0.001$, propionate $\mathrm{t}=120 P=0.015$, butyrate $\mathrm{t}=120 P=0.011$ ) and from time point $\mathrm{t}=60$ onwards for ${ }^{13} \mathrm{CO}_{2}$ (all time points $P<0.05$ ). As can be seen from Figure 3.6, the INU treatment caused more plasma butyrate to be labeled with ${ }^{13} \mathrm{C}(\mathrm{MPE}$ 0.36), than plasma acetate (MPE 0.17), or plasma propionate (MPE 0.14).

Figure 3.6. Plasma SCFA and exhaled $\mathrm{CO}^{2}$ enrichments

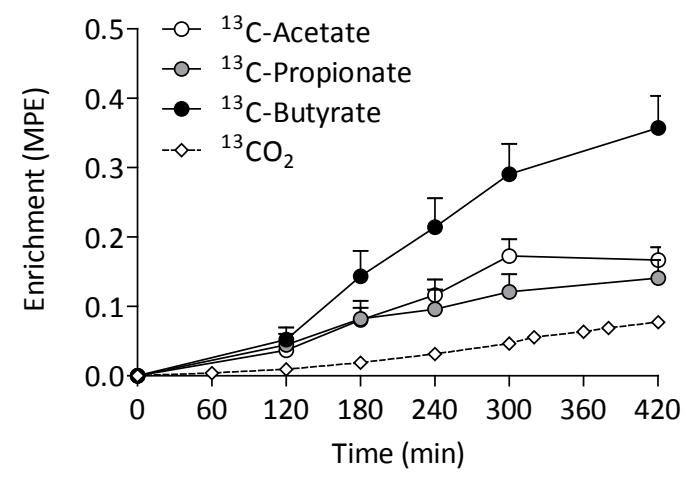

Mean $\left( \pm\right.$ SEM) plasma ${ }^{13} \mathrm{C}$-acetate, plasma ${ }^{13} \mathrm{C}$-propionate, plasma ${ }^{13} \mathrm{C}$-butyrate and exhaled ${ }^{13} \mathrm{CO}_{2}$ after consumption of inulin or placebo $(n=14)$. A repeated measures ANOVA showed a significant time effect $(P<0.05)$ for all three plasma ${ }^{13} \mathrm{C}-\mathrm{SCFAs}$, and a pairwise comparison showed this significant effect from time point $t=120$ onwards (Acetate $t=120 P<0.001$, propionate $\mathrm{t}=120 P=0.015$, butyrate $\mathrm{t}=120 P=0.011$ ). A repeated measures ANOVA showed a significant time effect $(P<0.05)$ for exhaled ${ }^{13} \mathrm{CO}_{2}$ from time point $\mathrm{t}=60$ onwards $\left({ }^{13} \mathrm{CO}_{2} \mathrm{t}=60\right.$ $P<0.001)$. Abbreviations: INU, Inulin; PLA, Placebo.

\section{Fecal SCFA concentrations and enrichments}

Fecal SCFA concentrations did not differ between the two groups (Figure 3.7A). ${ }^{13} \mathrm{C}$-enrichment in the INU group showed an enrichment of $0.31 \pm 0.02$ for acetate, $0.38 \pm 0.02$ for propionate and $0.30 \pm 0.02$ for butyrate (Figure 3.7B). One placebo fecal sample was tested and as expected showed no enrichment (data not shown).

\section{VAS scoring on hunger and appetite}

No significant differences in VAS scores on hunger and appetite were observed between treatments in the early or late postprandial phase. For both treatments, satiety and fullness increased directly after the intake of the high-fat milkshake containing the INU or PLA, while hunger and desire to eat decreased immediately after intake of the milkshake (Figure 3.8A-D). 
Figure 3.7. Fecal SCFA concentrations and enrichments

A

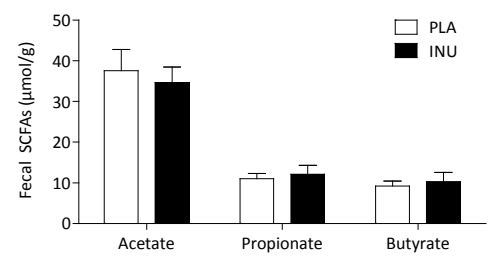

B

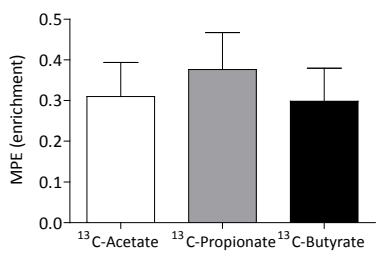

Mean ( \pm SEM) fecal SCFA concentration (A) and fecal SCFA enrichment (B) after consumption of inulin or placebo $(n=14)$. There were no significant differences between the two treatments. For ${ }^{13} \mathrm{C}$-enrichment, only the inulin group was tested. Abbreviations: INU, Inulin; PLA, Placebo; SCFA, Short-chain fatty acid.

Figure 3.8. VAS scores

A

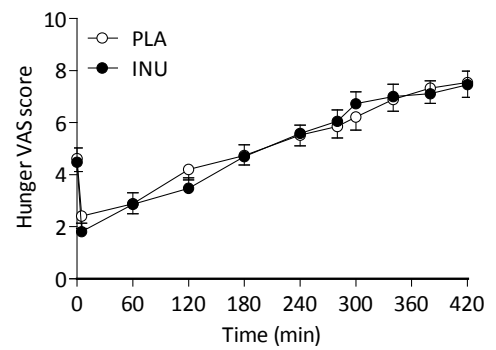

C

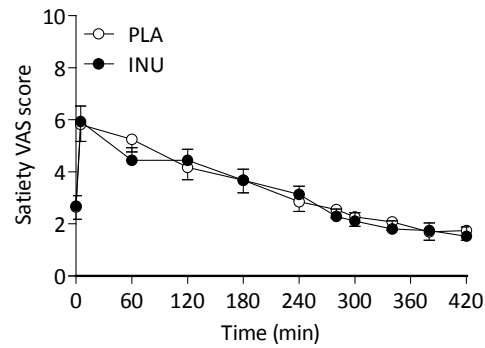

B

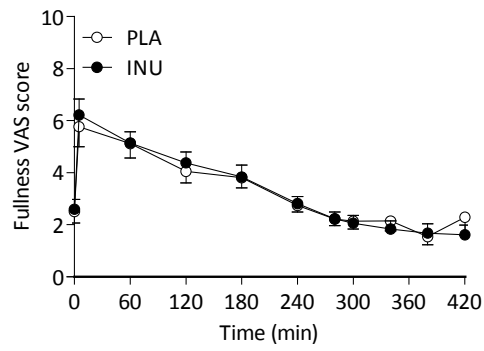

D

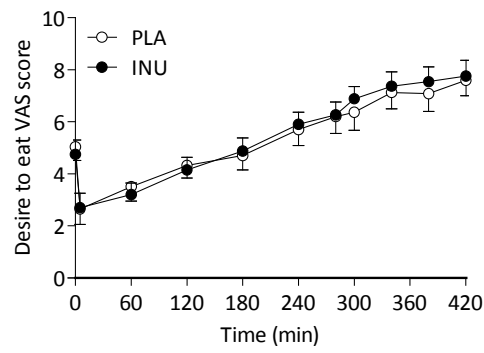

VAS scores on hunger (A), fullness (B), satiety (C), and desire to eat (D) after consumption of inulin or placebo $(n=14)$. There were no significant differences between treatments. Abbreviations: INU, Inulin; PLA, Placebo; VAS, Visual Analogue Scale. 


\section{DISCUSSION}

The present study investigated the acute metabolic effects of the ingestion of a single $24 \mathrm{~g}$ dose of the prebiotic inulin compared with digestible maltodextrin in a high-fat milkshake in overweight to obese subjects. We observed higher fat oxidation and a reduced postprandial decrease in plasma FFAs in the early postprandial phase after inulin ingestion. Also, inulin ingestion resulted in lower early postprandial plasma glucose and insulin concentrations compared with the placebo maltodextrin. In addition, inulin intake increased plasma acetate concentrations and lower plasma FFA concentrations in the late (6 - $7 \mathrm{~h}$ ) postprandial period. Tracer methodology revealed that ${ }^{13} \mathrm{C}$-labeled inulin is fermented into SCFAs as indicated by ${ }^{13} \mathrm{CO}_{2}$ in exhaled breath as well as appearance of ${ }^{13} \mathrm{C}$-labeled SCFAs in the systemic circulation from $2 \mathrm{~h}$ after inulin intake.

The improvement in substrate metabolism after inulin ingestion can be related to the lower glycemic index of inulin compared with maltodextrin. The glycemic index of a carbohydrate determines to what extent blood glucose concentrations rise upon ingestion. Orally ingested digestible carbohydrates are quickly digested and absorbed in the small intestine and become available as glucose in the systemic circulation. An increase in plasma glucose results in insulin release, which inhibits lipolysis. Fermentable carbohydrates such as inulin are not digested and do not increase blood glucose concentrations upon ingestion. In agreement, we show that blood glucose and insulin are lower in the early postprandial phase after the ingestion of inulin when compared with the digestible carbohydrate maltodextrin. As such, lipolysis is inhibited to a lesser extent, resulting in an attenuated postprandial decline in plasma FFAs and a higher fat oxidation. In addition to the higher glycemic index, digestible carbohydrates have an higher energy content than fermentable fibers. Nonetheless, replacing maltodextrin by inulin did not affect hunger and satiety, as indicated by the VAS scores (Figure 3.8). Low-glycemic index nutrients such as inulin can be used to modulate the postprandial glycemic response as well as energy intake, and thereby may improve insulin sensitivity and obesity-related symptoms. ${ }^{32}$

The second mechanism by which inulin can improve substrate metabolism is by microbial fermentation of inulin into SCFAs. The use of stable isotope-labeled inulin allowed us to assess the metabolic fate of ingested inulin. We demonstrated that inulin is fermented into all three SCFAs, as indicated by the postprandial appearance of ${ }^{13} \mathrm{C}$-acetate, ${ }^{13} \mathrm{C}$-propionate, and ${ }^{13} \mathrm{C}$-butyrate in plasma. Inulin-derived SCFAs were partly absorbed by the intestine and reached the systemic circulation (Figure 3.6). Via the systemic circulation SCFAs become available to metabolically active organs such as the liver and adipose tissue where they can promote substrate metabolism. In addition, part of the SCFAs was excreted, as indicated by the presence of ${ }^{13} \mathrm{C}$-SCFAs in the feces (Figure 3.7B). The relative abundance of inulin-derived (exogenous) ${ }^{13} \mathrm{C}$-butyrate in the circulation was shown to be greater compared 
with ${ }^{13} \mathrm{C}$-propionate and ${ }^{13} \mathrm{C}$-acetate. Plasma acetate concentrations - compiled of both endogenous and exogenous acetate - were higher after inulin treatment compared with maltodextrin placebo. This is in line with in vitro studies showing that inulin shifts SCFA production towards an increase in acetate, compared with propionate and butyrate in a 65:20:15 ratio, respectively. ${ }^{11,12,33}$ Moreover, Boets et al. ${ }^{34}$ showed in young healthy individuals - using continuous infusion of stable isotope-labeled SCFAs - that ingestion of $15 \mathrm{~g}$ inulin leads to cumulative amounts of $55 \pm 30 \mathrm{mmol}$ acetate, $1.1 \pm 0.9 \mathrm{mmol}$ propionate, and $1.0 \pm 0.9 \mathrm{mmol}$ butyrate appearing in plasma. However, as it has been postulated that a different microbial composition between lean and obese volunteers accounts for a different fermentation profile of dietary fibers, ${ }^{35}$ it is possible that the fermentation profile is different in our overweight to obese population.

Our research group has recently shown that colonic administration of acetate improves fat oxidation in overweight subjects. ${ }^{7}$ In addition, consumption of prebiotics or colonic SCFA administration reduces plasma FFA concentrations in both healthy and hyperinsulinemic subjects. ${ }^{23,36,37}$ In the present study, we show in the present study that ingestion of the prebiotic inulin increases fat oxidation (early phase) and reduces plasma FFAs (late phase). Though we cannot prove a causal relationship, it seems possible that the production of SCFAs from inulin ingestion is, at least partly, responsible for the increase in fat oxidation and the reduction in plasma FFAs. Lowering plasma FFAs may improve long-term glucose control, since high FFA concentrations are associated with peripheral and hepatic insulin resistance. ${ }^{38}$ Moreover, SCFAs can improve glycemia by binding to colonic GPR receptors leading to release of the gut hormones PYY and GLP-1. PYY and GLP-1 promote glucose storage in muscle and adipose tissue. ${ }^{39,40}$ However, we observed no differential increase in plasma PYY and GLP-1 in the inulin treatment compared with placebo. Therefore, it seems unlikely that the glucose/insulin-lowering effect of inulin in the present study can mechanistically be attributed to an increase in gut hormones by SCFA stimulation, but is rather an effect of the lower glycemic index of inulin.

The early postprandial increase in fat oxidation was accompanied by an immediate increase in breath ${ }^{13} \mathrm{CO}_{2}$ and plasma ${ }^{13} \mathrm{C}$-SCFAs. Stable isotope-labeled inulin was used to trace inulin-derived SCFAs, which revealed that fermentation of inulin is initiated in the early postprandial phase from $2 \mathrm{~h}$ after intake. Therefore, it seems likely that a combination of the lower glycemic index of inulin and the production of SCFAs through fermentation of inulin contributes to the observed improvements in substrate metabolism. Our observation is in line with a study by Rahat-Rozenbloom et al. ${ }^{41}$, showing an increase in plasma SCFA concentrations at $2 \mathrm{~h}$ after inulin intake paralleled by an increase in breath hydrogen from the same time point, indicating fermentation of inulin from $2 \mathrm{~h}$ onwards. Conversely, Tarini et al. ${ }^{23}$ found an increase in plasma SCFAs between 4 and $7 \mathrm{~h}$ after inulin intake in a lean, healthy population. An early increase in plasma ${ }^{13} \mathrm{C}$-SCFAs suggests inulin fermentation in the upper gastrointestinal tract. However, in vitro and in vivo studies have shown 
that inulin remains intact while passing through the small intestine, providing evidence that inulin is mainly fermented in the colon.42-44 Importantly, obese individuals have an increased gastric emptying rate and faster intestinal transit. ${ }^{45-46}$ As such, dietary inulin might reach the colon more rapidly in obese compared with lean individuals, where it is fermented into SCFAs that are released into the circulation and subsequently cause an early effect on human metabolism.

Our data implicate that replacing digestible carbohydrates with fermentable fibers may improve metabolic health in overweight to obese individuals. Future studies could implement the use of inulin as a more practical approach to increase SCFA availability and assess its effectiveness on markers of the metabolic syndrome. Moreover, the use of recently developed inulin-SCFA esters, whereby inulin serves as a vector to increase the amount of SCFAs, is an interesting approach for future studies to further increase fat oxidation and improve human metabolism. ${ }^{47}$ In addition, more studies should focus on overweight and obese individuals, since this target population might have different postprandial responses than a lean population.

In conclusion, ingestion of the prebiotic inulin improves fat oxidation and promotes SCFA production in overweight to obese men. The lower glycemic index of inulin and its SCFA-producing property might be responsible for the improvement in human metabolism. Replacing digestible carbohydrates with the fermentable inulin may favor human substrate metabolism. 


\section{REFERENCES}

1. Ng M, Fleming T, Robinson M, et al. Global, regional, and national prevalence of overweight and obesity in children and adults during 1980-2013: a systematic analysis for the Global Burden of Disease Study 2013. Lancet 2014;384:766-81.

2. Rabot S, Membrez M, Bruneau A, et al. Germ-free C57BL/6J mice are resistant to highfat-diet-induced insulin resistance and have altered cholesterol metabolism. FASEB J 2010;24:4948-59.

3. Diamant $M$, Blaak EE, de Vos WM. Do nutrient-gut-microbiota interactions play a role in human obesity, insulin resistance and type 2 diabetes? Obes Rev 2011;12:272-81.

4. Backhed F, Ding $\mathrm{H}$, Wang $\mathrm{T}$, et al. The gut microbiota as an environmental factor that regulates fat storage. Proc Natl Acad Sci U S A 2004;101:15718-23.

5. Gibson GR, Roberfroid MB. Dietary modulation of the human colonic microbiota: introducing the concept of prebiotics. J Nutr 1995;125:1401-12.

6. Canfora EE, Jocken JW, Blaak EE. Short-chain fatty acids in control of body weight and insulin sensitivity. Nat Rev Endocrinol 2015.

7. van der Beek CM, Canfora EE, Lenaerts K, et al. Distal, not proximal, colonic acetate infusions promote fat oxidation and improve metabolic markers in overweight/obese men. Clinical science 2016;130:2073-2082.

8. Capriles VD, Areas JA. Effects of prebiotic inulin-type fructans on structure, quality, sensory acceptance and glycemic response of gluten-free breads. Food \& function 2013;4:104-10.

9. Perry RJ, Peng L, Barry NA, et al. Acetate mediates a microbiome-brain-beta-cell axis to promote metabolic syndrome. Nature 2016;534:213-7.

10. Roberfroid MB, Delzenne NM. Dietary fructans. Annu Rev Nutr 1998;18:117-43.

11. Timm DA, Stewart ML, Hospattankar A, et al. Wheat dextrin, psyllium, and inulin produce distinct fermentation patterns, gas volumes, and short-chain fatty acid profiles in vitro. J Med Food 2010;13:961-6.

12. Kaur A, Rose DJ, Rumpagaporn P, et al. In vitro batch fecal fermentation comparison of gas and short-chain fatty acid production using "slowly fermentable" dietary fibers. Journal of food science 2011;76:H137-42.

13. Wang X, Gibson GR. Effects of the in vitro fermentation of oligofructose and inulin by bacteria growing in the human large intestine. J Appl Bacteriol 1993;75:373-80.

14. Gibson GR, Beatty ER, Wang X, et al. Selective stimulation of bifidobacteria in the human colon by oligofructose and inulin. Gastroenterology 1995;108:975-82.

15. Brighenti F, Casiraghi MC, Canzi E, et al. Effect of consumption of a ready-to-eat breakfast cereal containing inulin on the intestinal milieu and blood lipids in healthy male volunteers. Eur J Clin Nutr 1999;53:726-33.

16. Pourghassem Gargari B, Dehghan P, Aliasgharzadeh A, et al. Effects of high performance inulin supplementation on glycemic control and antioxidant status in women with type 2 diabetes. Diabetes Metab J 2013;37:140-8.

17. Letexier D, Diraison F, Beylot M. Addition of inulin to a moderately high-carbohydrate diet reduces hepatic lipogenesis and plasma triacylglycerol concentrations in humans. The American journal of clinical nutrition 2003;77:559-64. 
18. Russo F, Chimienti G, Riezzo G, et al. Inulin-enriched pasta affects lipid profile and Lp(a) concentrations in Italian young healthy male volunteers. European journal of nutrition 2008;47:453-9.

19. Russo F, Clemente $C$, Linsalata $M$, et al. Effects of a diet with inulin-enriched pasta on gut peptides and gastric emptying rates in healthy young volunteers. European journal of nutrition 2011;50:271-7.

20. Jackson KG, Taylor GR, Clohessy AM, et al. The effect of the daily intake of inulin on fasting lipid, insulin and glucose concentrations in middle-aged men and women. The British journal of nutrition 1999;82:23-30.

21. Davidson MH, Maki KC. Effects of dietary inulin on serum lipids. J Nutr 1999;129:1474S-7S.

22. Russo F, Riezzo G, Chiloiro M, et al. Metabolic effects of a diet with inulin-enriched pasta in healthy young volunteers. Current pharmaceutical design 2010;16:825-31.

23. Tarini J, Wolever TM. The fermentable fibre inulin increases postprandial serum short-chain fatty acids and reduces free-fatty acids and ghrelin in healthy subjects. Appl Physiol Nutr Metab 2010;35:9-16.

24. Weir JB. New methods for calculating metabolic rate with special reference to protein metabolism. The Journal of physiology 1949;109:1-9.

25. Frayn KN. Calculation of substrate oxidation rates in vivo from gaseous exchange. Journal of applied physiology: respiratory, environmental and exercise physiology 1983;55:628-34.

26. Orskov C, Rabenhoj L, Wettergren A, et al. Tissue and plasma concentrations of amidated and glycine-extended glucagon-like peptide I in humans. Diabetes 1994;43:535-9.

27. van Eijk HM, Bloemen JG, Dejong CH. Application of liquid chromatography-mass spectrometry to measure short chain fatty acids in blood. Journal of chromatography. B, Analytical technologies in the biomedical and life sciences 2009;877:719-24.

28. Boets E, Gomand SV, Deroover L, et al. Systemic availability and metabolism of colonic-derived short-chain fatty acids in healthy subjects: a stable isotope study. The Journal of physiology 2016.

29. Morrison DJ, Cooper K, Waldron S, et al. A streamlined approach to the analysis of volatile fatty acids and its application to the measurement of whole-body flux. Rapid communications in mass spectrometry: RCM 2004;18:2593-600.

30. Garcia-Villalba R, Gimenez-Bastida JA, Garcia-Conesa MT, et al. Alternative method for gas chromatography-mass spectrometry analysis of short-chain fatty acids in faecal samples. Journal of separation science 2012;35:1906-13.

31. Higgins JA, Higbee DR, Donahoo WT, et al. Resistant starch consumption promotes lipid oxidation. Nutr Metab (Lond) 2004;1:8.

32. Latulippe ME, Meheust A, Augustin L, et al. ILSI Brazil International Workshop on Functional Foods: a narrative review of the scientific evidence in the area of carbohydrates, microbiome, and health. Food \& nutrition research 2013;57.

33. Hughes SA, Shewry PR, Li L, et al. In vitro fermentation by human fecal microflora of wheat arabinoxylans. Journal of agricultural and food chemistry 2007;55:4589-95.

34. Boets E, Deroover L, Houben E, et al. Quantification of in Vivo Colonic Short Chain Fatty Acid Production from Inulin. Nutrients 2015;7:8916-29.

35. Aguirre M, Bussolo de Souza C, Venema K. The Gut Microbiota from Lean and Obese Subjects Contribute Differently to the Fermentation of Arabinogalactan and Inulin. PloS one 2016;11:e0159236. 
36. Wolever TM, Brighenti F, Royall D, et al. Effect of rectal infusion of short chain fatty acids in human subjects. The American journal of gastroenterology 1989;84:1027-33.

37. Fernandes J, Vogt J, Wolever TM. Inulin increases short-term markers for colonic fermentation similarly in healthy and hyperinsulinaemic humans. European journal of clinical nutrition 2011;65:1279-86.

38. Wolever TM, Bentum-Williams A, Jenkins DJ. Physiological modulation of plasma free fatty acid concentrations by diet. Metabolic implications in nondiabetic subjects. Diabetes care 1995;18:962-70.

39. Batterham RL, Cowley MA, Small C), et al. Gut hormone PYY(3-36) physiologically inhibits food intake. Nature 2002;418:650-4.

40. MacDonald PE, El-Kholy W, Riedel MJ, et al. The multiple actions of GLP-1 on the process of glucose-stimulated insulin secretion. Diabetes 2002;51 Suppl 3:5434-42.

41. Rahat-Rozenbloom S, Fernandes J, Cheng J, et al. The acute effects of inulin and resistant starch on postprandial serum short-chain fatty acids and second-meal glycemic response in lean and overweight humans. European journal of clinical nutrition 2016.

42. Bach Knudsen KE, Hessov I. Recovery of inulin from Jerusalem artichoke (Helianthus tuberosus L.) in the small intestine of man. Br J Nutr 1995;74:101-13.

43. Ellegard L, Andersson H, Bosaeus I. Inulin and oligofructose do not influence the absorption of cholesterol, or the excretion of cholesterol, Ca, Mg, Zn, Fe, or bile acids but increases energy excretion in ileostomy subjects. Eur J Clin Nutr 1997;51:1-5.

44. Van den Abbeele P, Venema K, Van de Wiele T, et al. Different human gut models reveal the distinct fermentation patterns of Arabinoxylan versus inulin. Journal of agricultural and food chemistry 2013;61:9819-27.

45. Mushref MA, Srinivasan S. Effect of high fat-diet and obesity on gastrointestinal motility. Ann Transl Med 2013;1:14.

46. Wisen O, Johansson C. Gastrointestinal function in obesity: motility, secretion, and absorption following a liquid test meal. Metabolism 1992;41:390-5.

47. Chambers ES, Viardot A, Psichas A, et al. Effects of targeted delivery of propionate to the human colon on appetite regulation, body weight maintenance and adiposity in overweight adults. Gut 2015;64:1744-54. 


\section{SUPPLEMENTAL FIGURE}

Supplemental Figure 3.1. Flow chart of human subjects included in this study

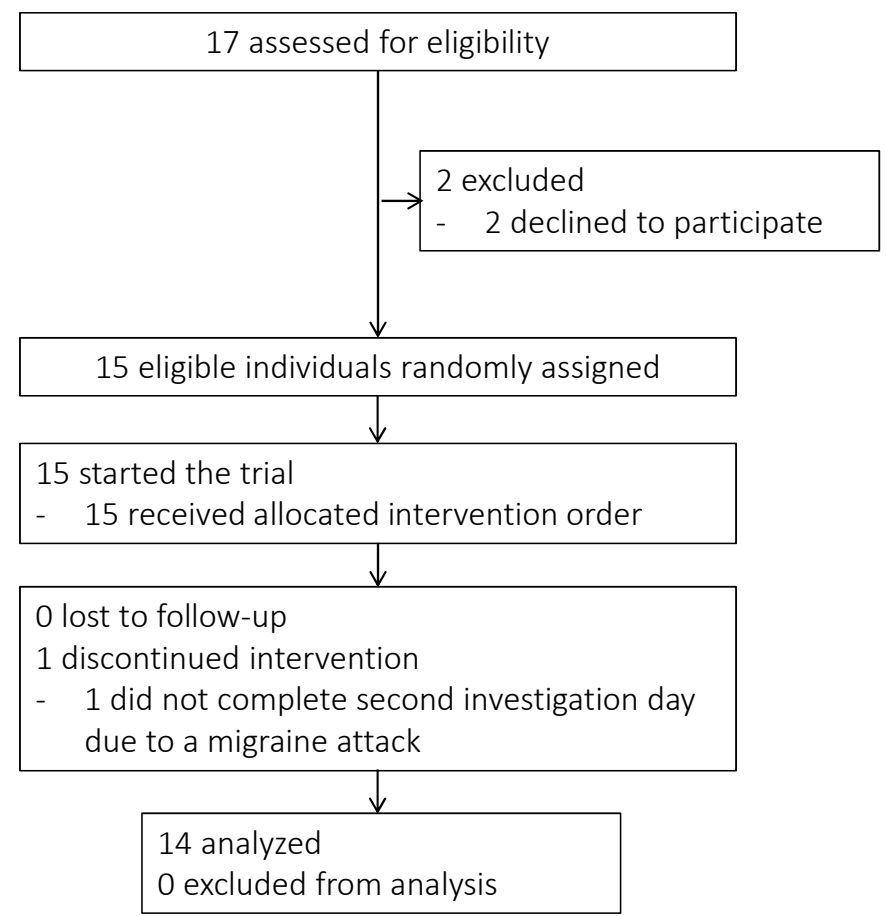





$$
4
$$




\section{CHAPTER 4}

\section{Supplementation of diet with galacto-oligosaccharides increases bifidobacteria, but not insulin sensitivity, in obese prediabetic individuals}

Emanuel E. Canfora*, Christina M. van der Beek*, Gerben D. A. Hermes, Gijs H.

Goossens, Johan W. E. Jocken, Jens J. Holst, Hans M. van Eijk, Koen Venema,

Hauke Smidt, Erwin G. Zoetendal, Cornelis H. C. Dejong, Kaatje Lenaerts, Ellen E. Blaak

*Both authors contributed equally 


\section{ABSTRACT}

Background and aims: The gut microbiota affects host lipid and glucose metabolism, satiety, and chronic low-grade inflammation to contribute to obesity and type 2 diabetes. Fermentation end products, in particular the short-chain fatty acid (SCFA) acetate, are believed to be involved in these processes. We investigate the long-term effects of supplementation with galacto-oligosaccharides (GOS), an acetogenic fiber, on the composition of the human gut microbiota and human metabolism.

Methods: We performed a double-blinded, placebo-controlled, parallel intervention study of 44 overweight or obese (body mass index, $28-40 \mathrm{~kg} / \mathrm{m}^{2}$ ) prediabetic men and women (ages, 45 - 70 y) from October 2014 through October 2015 in Maastricht, The Netherlands. The participants were randomly assigned to groups who ingested $15 \mathrm{~g}$ GOS or isocaloric placebo (maltodextrin) daily with their regular meals for twelve weeks. Before and after this period, we collected data on peripheral and adipose tissue insulin sensitivity, fecal microbiota composition, plasma and fecal SCFA, energy expenditure and substrate oxidation, body composition, and hormonal and inflammatory responses. The primary outcome was the effect of GOS on peripheral insulin sensitivity, measured by the hyperinsulinemiceuglycemic clamp method.

Results: Supplementation of diets with GOS, but not placebo, increased by 5-fold the abundance of Bifidobacterium spp. in feces ( $P=0.009, q=0.144$ ). Microbial richness or diversity in fecal samples were not affected. We did not observe any differences in fecal or fasting plasma SCFA concentrations or in systemic concentrations of gut-derived hormones, incretins, lipopolysaccharide-binding protein, or other markers of inflammation. In addition, no significant alterations in peripheral and adipose tissue insulin sensitivity, body composition, and energy and substrate metabolism, were found.

Conclusions: Twelve-week supplementation of GOS selectively increased fecal Bifidobacterium spp. abundance, but this did not produce significant changes in insulin sensitivity or related substrate and energy metabolism in overweight or obese prediabetic men and women. 


\section{INTRODUCTION}

Accumulating evidence indicates that the human gut microbiota is involved in the etiology of obesity and type 2 diabetes. ${ }^{1-3}$ Several strategies to manipulate gut microbiota composition including fecal transplantation, antibiotic treatment, as well as supplementation of probiotics and prebiotics indicated that alterations in the microbial composition and diversity might lead to changes in insulin sensitivity and metabolic profile in humans, ${ }^{4 \cdot 7}$ although data are not consistent.8-10 Importantly, however, long-term dietary intervention studies in humans, combining microbiota analysis with detailed metabolic phenotyping are scarce, and putative underlying mechanisms for beneficial effects on host metabolism remain to be fully elucidated.

Many data on prebiotic effects of dietary fibers are derived from studies with either inulin-type fructo-oligosaccharides (FOS) or galacto-oligosaccharides (GOS). ${ }^{11}$ These dietary fibers have the capacity to selectively alter the gut microbiota composition and stimulate the growth of putatively beneficial bacterial genera such as Bifidobacterium and Lactobacillus. ${ }^{11,12}$ GOS is a soluble dietary fiber derived from $\beta$-galactosidase-induced conversion of lactose. A limited number of human studies have indicated beneficial metabolic effects of GOS. ${ }^{13,14}$ Twelve-week supplementation of a GOS mixture to overweight participants improved insulin and lipid homeostasis, and attenuated low-grade systemic inflammation in these participants. ${ }^{13}$ Furthermore, dietary supplementation of a GOS mixture to healthy, elderly volunteers altered gut microbiota composition and improved systemic and fecal inflammatory markers. ${ }^{14}$ However, mechanisms involved in these metabolic effects were not determined in these studies. One of the important mechanisms involved in the effects of prebiotic fibers on metabolic health is their fermentation by the gut microbiota, resulting in the formation of short-chain fatty acids (SCFAs), mainly acetate, propionate, and butyrate. ${ }^{15}$

We have recently demonstrated that acute infusion of acetate into the distal part of the colon led to increased systemic acetate concentrations, increased fat oxidation, and circulating concentrations of the satiety-stimulating hormone peptide YY (PYY) in overweight men. ${ }^{16}$ Based on that, we hypothesized that long-term supplementation of a high acetogenic fiber would result in increased circulating acetate availability and an improved metabolic profile. Increased acetate concentrations were found after in vitro fermentation of GOS. ${ }^{17}$ In accordance, a six-week supplementation of GOS to infants increased fecal acetate concentrations and fecal abundance of Bifidobacterium. ${ }^{18}$ Several members of colonic microbiota are involved in GOS fermentation, of which bifidobacteria are mainly responsible. ${ }^{19}$ As such, bifidobacteria convert polysaccharides into mainly lactate and acetate. ${ }^{20}$ Therefore, long-term GOS intake may beneficially modulate the gut microbiota composition and induce acetate production, leading to improved host energy, and improved 
substrate metabolism and insulin sensitivity. Hence, the present study investigated the effects of twelve-week GOS supplementation on peripheral insulin sensitivity in overweight and obese, prediabetic men and postmenopausal women. Secondary outcomes were effects of GOS supplementation on fecal microbiota composition, plasma and fecal SCFA concentrations, body composition, energy expenditure and substrate oxidation, circulating metabolites, hormones, inflammatory markers, and adipokine concentrations.

\section{METHODS}

\section{Study participants}

Forty-six overweight and obese (body mass index, BMI 28 - 40 kg/m²) Caucasian men and postmenopausal women, aged 45 - $70 \mathrm{y}$, with impaired fasting glucose (IFG) and/or impaired glucose tolerance (IGT) were recruited between October 2014 and October 2015 from the vicinity of Maastricht, The Netherlands. IFG was defined as fasting plasma glucose concentrations $\geq 5.6 \mathrm{mmol} / \mathrm{L}$. IGT was defined as plasma glucose concentrations between $7.8-11 \mathrm{mmol} / \mathrm{L}$ at $2 \mathrm{~h}$ after oral ingestion of $75 \mathrm{~g}$ glucose dissolved in $250 \mathrm{~mL}$ tap water (oral glucose tolerance test, OGTT). Volunteers had to be weight stable for at least three months prior to study participation. Exclusion criteria for participation in the study were: diagnosis of diabetes mellitus, gastroenterological diseases or prior abdominal surgery, cardiovascular diseases, liver or kidney malfunction, patients with a life expectancy shorter than $5 \mathrm{y}$, participants following a hypocaloric diet, or use of antibiotics, pre- or probiotics in the three months prior to start of the study or during the study period. Participants did not use $\beta$-blockers, lipid and glucose lowering-drugs, anti-oxidants or chronic corticosteroids. The study was approved by the Medical Ethical Committee of Maastricht University Medical Centre+ (MUMC+) and was conducted in accordance with the Declaration of Helsinki (revised version, October 2008, Seoul, South Korea). Written informed consent was obtained from all participants. All authors had access to the study data and reviewed and approved the final manuscript.

\section{Study design}

This study was a double-blind, placebo-controlled, randomized, parallel trial. After stratification for sex and age, an independent researcher randomized subjects into the GOS or placebo group. Participants in the intervention group were asked to ingest $7.04 \mathrm{~g}$ of Vivinal® GOS powder (FrieslandCampina Domo, Amersfoort, the Netherlands), containing $5 \mathrm{~g}$ GOS, three times per day with their regular meals during the twelve-week intervention period (Figure 4.1). Besides 69\% GOS, the product contained 23\% lactose, 5\% monosaccharides (glucose and galactose), and 3\% moisture. Participants in the placebo group were asked to ingest $5.65 \mathrm{~g}$ of maltodextrin (Avebe, Veendam, the Netherlands) three 
times per day with their regular meals during twelve weeks. The amount of maltodextrin was isocaloric to the amount of Vivinal® GOS powder product (269.6 kJ per day). GOS and maltodextrin were both provided as white powdered supplements. The products were provided in sachets and were consumed with a low fat yogurt drink (Optimel Drink Lang Lekker 200 mL, FrieslandCampina, Amersfoort, the Netherlands). There were no probiotic strains or supplemented GOS in the yogurt drink. The participants were instructed to document their GOS or placebo intake in a daily diary and had to return all sachets to assess compliance. All participants were instructed to continue their usual physical activity and dietary regimen throughout the whole intervention period to study the isolated effect of the differential supplementation.

Primary outcome of the study was the effect of GOS on peripheral insulin sensitivity as measured by the hyperinsulinemic-euglycemic clamp method. Secondary outcomes were substrate oxidation and energy expenditure, fecal microbiota composition, fecal and plasma SCFAs, circulating metabolites (glucose, triacylglycerol (TAG), free fatty acids (FFAs), free glycerol) and hormones (insulin, PYY, glucagon-like peptide-1 (GLP-1), leptin), plasma inflammatory markers (tumor necrosis factor-a (TNF-a), interleukin-6 (IL-6), interleukin-8 (IL-8), lipopolysaccharide binding protein (LBP)), body composition, BMI, body weight, dietary intake and physical activity.

Figure 4.1. Outline of study design

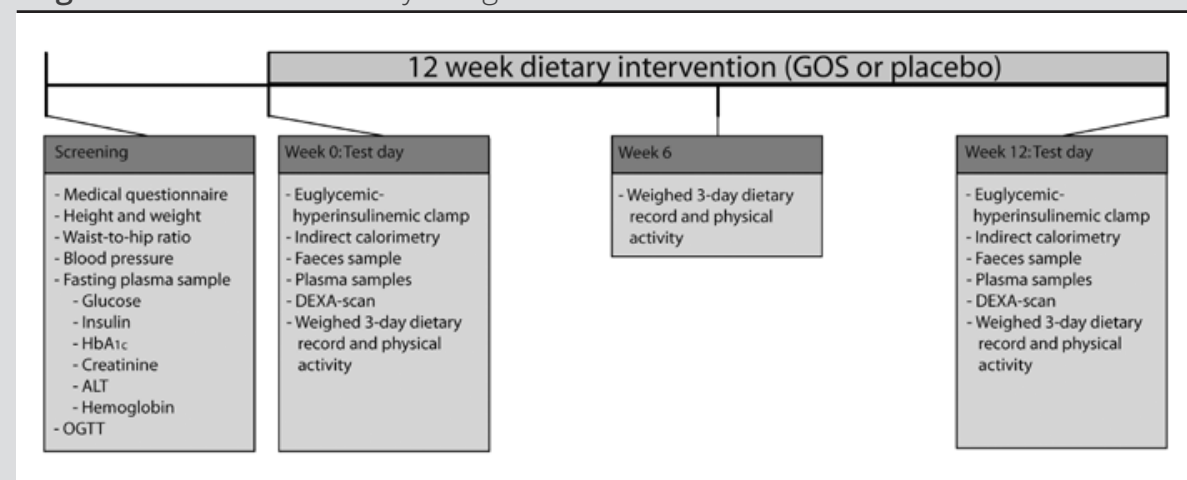

Abbreviations: ALT, alanine transaminase; DEXA, dual energy X-ray absorptiometry-scanning; OGTT, oral glucose tolerance test; GOS, galacto-oligosaccharides; HbA ${ }_{1 c^{\prime}}$ glycated hemoglobin. 
Before and directly after the twelve-week intervention period, participants underwent an experimental clinical investigation day (CID). The twelve-week intervention started the day after the first CID. During the three days prior to the CID participants recorded their dietary intake and level of physical activity. In addition, fecal samples were collected one day prior to the CID.

\section{Dietary intake and physical activity recording}

Participants were asked to complete a three-day dietary record before the intervention period, in week 6 and in week 12 of the intervention. Before start of the intervention, participants were instructed by a dietician how to weigh and record their food and beverage intake. The volunteers were asked to include two week days and one weekend day in the three-day period. The dietary records were checked, discussed, in case of missing data, and analyzed by an experienced dietician. Energy and nutrient intake were analyzed using the Dutch Food Composition Dataset (NEVO, National Institute for Public Health and Environment, Ministry of Health, Welfare and Sport, The Hague, The Netherlands).

Self-reported level of physical activity was assessed using the Short Questionnaire to Assess Health enhancing physical activity (SQUASH). ${ }^{21}$ Participants completed the questionnaire before the intervention period and in week 6 and week 12 of the intervention period. Outcome value was the time spent (min) in light, moderate, and vigorous physical activity based on metabolic equivalent (MET), as reported before.21

\section{Clinical investigation day (CID)}

Two days prior to the CID, participants were asked to refrain from intense physical activity and alcohol consumption. In the evening before each CID, the volunteers consumed a standardized low fiber meal (62 energy\% carbohydrate, 24 energy\% protein, and 14 energy\% fat). Participants came to the laboratory by car or public transport in the morning after an overnight fast (12 h). All procedures were carried out with the participant in a resting, half-supine position. During the CID, several measurements took place. Each CID started with a dual energy X-ray absorptiometry-scan (DEXA) to determine body composition and measurements of body weight and height. Subsequently, a one-step hyperinsulinemic-euglycemic clamp was performed to measure peripheral insulin sensitivity, combined with indirect calorimetry to measure substrate oxidation and energy expenditure during fasting and euglycemic conditions. In addition, blood samples were taken at different time points during fasting and euglycemic conditions (Figure 4.1).

\section{Body composition}

Body fat percentage, body fat and visceral fat distribution, as well as lean mass were measured before and after the intervention period by use of a DEXA scan using the three-compartment model (Hologic BCA, VitaK, Maastricht, The Netherlands). In addition, 
fasting body weight (in underwear using a calibrated weight scale) and height (barefoot) were measured in order to calculate BMI.

\section{One step hyperinsulinemic-euglycemic clamp}

A hyperinsulinemic-euglycemic clamp was performed to measure whole-body insulin-stimulated glucose rate of disappearance. Participants were placed in a semi-recumbent position. First, a Teflon cannula was inserted into an antecubital vein for infusion of glucose and insulin. In order to measure blood glucose, a second Teflon cannula was inserted into a superficial dorsal hand vein for sampling of blood (1 $\mathrm{mL}$ every $5 \mathrm{~min}$ ), which was arterialized by placing the hand into a hotbox, blowing warm air $\left(\sim 50^{\circ} \mathrm{C}\right)$. A priming dose of insulin infusion (Actrapid, Novo Nordisk, Gentofte, Denmark) was administered during the first ten min (t0 - t10 min) and insulin infusion was thereafter continued at $40 \mathrm{mU} /$ $\mathrm{m}^{2} / \mathrm{min}$ for $2 \mathrm{~h}$ (t10 - t120 min). By variable infusion of a 17.5\% glucose solution, plasma concentrations were maintained at $5.0 \mathrm{mmol} / \mathrm{L}$. The mean glucose infusion rate during the final 30 min of euglycemia was used as an indicator of the peripheral insulin sensitivity and defined as the M-value.22 In addition, during fasting conditions (t-30 - t0 min) and during the 30 min steady state of the clamp (t90 - t120 min), energy expenditure and substrate oxidation were measured using indirect calorimetry. Additional blood plasma samples were taken during fasting (t-5 min) and euglycemic (t90 and t120 min) conditions.

Insulin-stimulated suppression of circulating FFA was measured as an indicator of adipose tissue insulin sensitivity. Insulin-stimulated FFA suppression in \% was calculated by the formula: ((baseline FFA - insulin stimulated FFA during steady state clamp) / baseline FFA). ${ }^{23}$

\section{Indirect calorimetry}

For indirect calorimetry, an open-circuit ventilated hood system (Omnical, MUMC+, Maastricht, the Netherlands) was used. $\mathrm{CO}_{2}$ production $\left(\mathrm{VCO}_{2}\right.$ in $\mathrm{L} / \mathrm{min}$ ) and $\mathrm{O}_{2}$ consumption $\left(\mathrm{VO}_{2}\right.$ in L/min) were measured during two investigational time periods: a baseline measurement of 30 min before start of the clamp (t-30 - t0 min) and for 30 min during the steady-state of the hyperinsulinemic-euglycemic clamp (t90 - t120 min). The equations of Weir ${ }^{24}$ and Frayn ${ }^{25}$ were used to calculate resting energy expenditure and the rate of fat and carbohydrate oxidation.

\section{Blood collection, storage and biochemical analyses}

Blood was collected into ice-cold EDTA tubes (0.2M EDTA (Sigma, Dorset, UK)) for SCFAs, insulin, glucose, FFAs, TAG, free glycerol, leptin, LBP, TNF-a, IL-6, and IL-8 analyses during fasting (t-5 min) and euglycemic (t90 and t120 min) conditions. For GLP-1 analysis, blood was collected in a $2 \mathrm{~mL}$ EDTA tube containing $20 \mu \mathrm{L}$ of dipeptidyl peptidase-IV inhibitor (Millipore, Darmstadt, Germany). For PYY analysis, blood was collected in a $2 \mathrm{~mL}$ aprotinin 
tube containing $20 \mu \mathrm{L}$ of dipeptidyl peptidase-IV inhibitor. The samples were centrifuged at 3,500 g, $4{ }^{\circ} \mathrm{C}$ for $10 \mathrm{~min}$, and plasma was aliquoted and directly snap-frozen in liquid nitrogen and stored at $-80{ }^{\circ} \mathrm{C}$ until analysis.

Plasma FFAs, TAG, and glucose were measured with enzymatic assays on an automated spectrophotometer (ABX Pentra 400 autoanalyzer, Horiba ABX, Montpellier, France). Plasma free glycerol was measured after precipitation with an enzymatic assay (EnzytecTM Glycerol, Roche Biopharm, Switzerland) automated on a Cobas Fara spectrophotometric autoanalyzer (Roche Diagnostics, Basel, Switzerland). The concentrations of insulin, leptin and PYY were determined with commercially available radioimmunoassay (RIA) kits (Human Insulin specific RIA, Human Leptin RIA, Human PYY (3-36) RIA, Millipore Corporation, MA, USA). IL-6, IL-8 and TNF-a were determined with an enzyme-linked immunosorbent assay (ELISA) kit (Human Prolnflammatory II 4-Plex Ultra-Sensitive Kit, Meso Scale Diagnostics, MD, USA). Plasma samples were assayed for total GLP-1 immunoreactivity using an anti-serum, which reacts equally with intact GLP-1 and the primary (N-terminally truncated) metabolite as previously described. ${ }^{26}$ For the detection of LBP, plates (Greiner Microlon 600 high binding) were coated with polyclonal anti-human LBP antibodies. Diluted plasma samples (1:5000) and a standard dilution series with rLBP were added to the plate. Detection occurred with a biotinylated polyclonal rabbit anti-human LBP IgG, followed by peroxidase-conjugated streptavidin and substrate. The detection limit for the LBP assay was 200 pg/mL. ${ }^{27}$

\section{SCFA analysis}

Feces was collected at home, starting from two days before the test days and stored in the subject's freezer at $-20^{\circ} \mathrm{C}$, transported on dry ice and stored on arrival at the university at $-80^{\circ} \mathrm{C}$. Fecal acetate, propionate, butyrate were measured by gas chromatography-mass spectrometry (GC-MS, Medical laboratory 'Dr. Stein \& Collegae', Mönchengladbach, Germany), according to the method described by Garciá-Villalba et al. ${ }^{28}$. Storage and processing of plasma samples for analysis of SCFA was performed as reported before. ${ }^{29}$ Analysis of these samples was done using liquid chromatography-mass spectrometry (LC-MS) enabling lower detection limits for acetate, propionate, and butyrate of 0.1, 0.05, and $0.05 \mu \mathrm{mol} / \mathrm{L}$, respectively.

\section{Microbiota composition}

DNA was isolated from feces using the repeated bead beating method as previously described, ${ }^{30}$ and subsequently used for microbiota profiling using the Human Intestinal Tract Chip (HITChip), a phylogenetic microarray based on 16S rRNA gene sequences of over 1000 intestinal bacterial phylotypes. ${ }^{31}$ In short, 16S rRNA genes were amplified by PCR, followed by in vitro transcription, Cy3/Cy5 labeling and fragmentation of RNA, and hybridization. Duplicate hybridizations with a Pearson correlation $>98 \%$ were con- 
sidered for further analysis, and microbiota profiles were generated by pre-processing of probe-level measurements with min-max normalization and the frozen-RPA probe summarization ${ }^{32}$ into three phylogenetic levels: order-like, genus-like (>90\% sequence similarity), and phylotype-like (>98\% sequence similarity). ${ }^{31}$ In the present work our analysis focused on the genus-level variation.

\section{Statistical analysis}

To detect a physiologically relevant difference in the change in peripheral insulin sensitivity of $20 \%$ with a SD of 4 , a power of $80 \%$ and assuming an alpha of $0.05(\alpha)$, a number of at least 17 participants per group was necessary. Since an equivalent subject number for men and women was required, 18 participants per group ( 9 men and 9 women per group) were needed. Assuming a $25 \%$ dropout rate, the planned recruitment was $n=46$ participants in total.

All data are expressed as mean \pm standard deviation (SD). Baseline differences were evaluated using a Student's unpaired t-test. Body composition data and metabolic variables were first tested for normality using the Shapiro-Wilk test and showed a normal distribution. A two-factor repeated-measures analysis of variance (ANOVA) with time (pre, post) and intervention (GOS, placebo) as factor was applied. In case of significant time $x$ intervention interaction, post-hoc analyses with Bonferroni correction were applied to identify significant within-intervention effects. Statistics were performed using SPSS 22.0 for Mac (Chicago, IL, USA) and $P<0.05$ (two-sided $P$-value) was considered statistically significant.

For HITChip analysis, log10-transformed signals were used as a proxy for bacterial logarithmic abundance. To determine effect of treatment on the abundance of genus-level microbial groups within individuals and the contrast between the two treatment groups, a linear mixed model taking into account the effects of repeated measurements, treatment group, age and BMI using the Ime4 package was used. ${ }^{33}$ Diversity of the microbiota was quantified based on non-logarithmized HITChip oligo-level signals by inverse Simpson's index using the Vegan package. ${ }^{34,35}$ ANOVA with Tukey's Honest Significant post-hoc analysis was applied to compare diversity between and within groups. P-values were corrected for multiple comparisons using the Benjamini-Hochberg procedure. HITChip statistics were performed using R v3.1.3 software. A corrected q-value of $<0.2$ was considered as significant.

\section{RESULTS}

Forty-six volunteers started with the twelve-week intervention period. Two women dropped out of the study due to the use of antibiotics during the study period (one for a wound infection after a bicycle fall and one for a lower respiratory tract infection, Supplemental 
Figure 4.1). Therefore, a total of 44 participants completed the study and their baseline characteristics are shown in Table 4.1. No significant differences were found between groups at baseline. Compliance was confirmed by counting returned empty sachets (245 \pm 11 of $251 \pm 7.6$ sachets (97.6\%) returned empty) in comparison with given amounts of the daily diary product. No adverse events were reported. Importantly, participants reported no side effects of GOS or placebo treatment, such as changes in stool frequency or gastrointestinal complaints.

Table 4.1. Study participants' baseline characteristics ( $n=44)$

\begin{tabular}{lccc}
\hline Variable & GOS $(\boldsymbol{n}=\mathbf{2 1})$ & Placebo $(\boldsymbol{n}=\mathbf{2 3})$ & $P$-value \\
\hline Gender (M/F) & $11 / 10$ & $12 / 11$ & \\
IGT / IFG / IGT + IFG ( $\mathrm{n})$ & $0 / 14 / 7$ & $2 / 15 / 6$ & \\
Age (y) & $59.2 \pm 7.2$ & $58.4 \pm 7.3$ & 0.716 \\
Weight (kg) & $98.4 \pm 11.9$ & $96.9 \pm 11.5$ & 0.263 \\
Height (cm) & $172.0 \pm 7.2$ & $173.5 \pm 7.5$ & 0.525 \\
Body mass index (kg/m²) & $33.3 \pm 3.7$ & $32.3 \pm 3.5$ & 0.321 \\
Waist-hip ratio & $1.0 \pm 0.06$ & $1.0 \pm 0.06$ & 0.899 \\
Systolic blood pressure (mmHg) & $129 \pm 11$ & $127 \pm 15$ & 0.742 \\
Diastolic blood pressure (mmHg) & $86 \pm 7$ & $85 \pm 10$ & 0.779 \\
Fasting glucose (mmol/L) & $6.0 \pm 0.5$ & $5.8 \pm 0.4$ & 0.095 \\
OGTT 2h plasma glucose (mmol/L) & $6.4 \pm 1.7$ & $7.0 \pm 2.0$ & 0.259 \\
Fasting insulin (mU/mL) & $20.7 \pm 6.7$ & $19.1 \pm 7.2$ & 0.721 \\
HOMA-IR & $5.34 \pm 2.7$ & $5.10 \pm 2.7$ & 0.829 \\
HbA1c (\%) & $5.6 \pm 0.3$ & $5.6 \pm 0.4$ & 0.509 \\
\hline Values &
\end{tabular}

Values are given as mean \pm SD. Data were analyzed using a Student's unpaired t-test. Abbreviations: IFG, impaired fasting glucose; IGT, impaired glucose tolerance; HOMA-IR, homeostatic model assessment of insulin resistance; OGTT, oral glucose tolerance test. 


\section{Fecal microbiota}

Twelve-week GOS supplementation consistently increased fecal Bifidobacterium spp. by $5.0 \pm 0.3$ fold ( $P=0.009, q=0.144$ ) (Figure 4.2A and 4.2B) compared to placebo. Other taxa whose abundance was differentially impacted by the GOS intervention compared to placebo were Prevotella oralis et rel. by $1.14 \pm 0.6$ fold ( $P=0.010, q=0.144)$ and Prevotella melaninogenica et rel. by $1.13 \pm 0.5$ fold $(P=0.008, q=0.144)$, Bacteroides stercoris et rel. by 0.83 \pm 0.2 fold $(P=0.011, q=0.144)$ and Sutterella wadsworthia et rel. by $0.85 \pm 0.1$ fold $(P=0.002$, $q=0.116$, figure 4.2A). However these taxa were all affected to a much smaller degree than Bifidobacterium spp. (Figure 4.2A) and showed a much less uniform pattern (Supplemental Figure 4.2A). The overall microbial richness $(P=0.307)$ and diversity $(P=0.626)$ were not different between groups (Supplemental Figure 4.2B and 4.2C).

\section{Insulin sensitivity}

Peripheral insulin sensitivity as assessed by the M-value was not changed after GOS treatment as compared with placebo ( $P=0.467$, Figure 4.3A and 4.3B). The Homeostasis Model (HOMA-IR) did not differ between treatments ( $P=0.598$, Figure 4.3C). In addition, insulin-stimulated FFA suppression, a measure for adipose tissue insulin sensitivity, was not affected by GOS as compared to placebo ( $P=0.808$, Figure 4.3D).

\section{Short-chain fatty acid concentrations}

Fecal and plasma acetate, propionate, and butyrate concentrations did not differ between intervention groups (Figure 4.4).

\section{Food intake and physical activity records}

Energy intake, macronutrient, micronutrient and dietary fiber intake, assessed using self-reported three-day food records, were not significantly different after twelve weeks GOS intervention compared to placebo (Supplemental table 4.1). Groups did not differ in physical activity levels as assessed via physical activity scores (Supplemental Table 4.1).

\section{Body composition, BMI and body weight}

BMI, body weight, body fat percentage, body fat mass, lean mass and visceral adipose tissue mass were not significantly affected by GOS supplementation compared to placebo (Table 4.2).

\section{Circulating metabolites, hormones and inflammatory profile}

No changes in fasting plasma glucose, insulin, glycerol, FFAs and TAG were observed between treatments (Table 4.3). Likewise fasting plasma concentrations of leptin, PYY, GLP-1, and the inflammatory markers IL-6, IL-8, TNF- $a$ and LBP were not significantly affected by the intervention as compared to placebo (Table 4.3). 
Figure 4.2. Gut microbiota composition before (pre) and after (post) GOS and placebo intervention

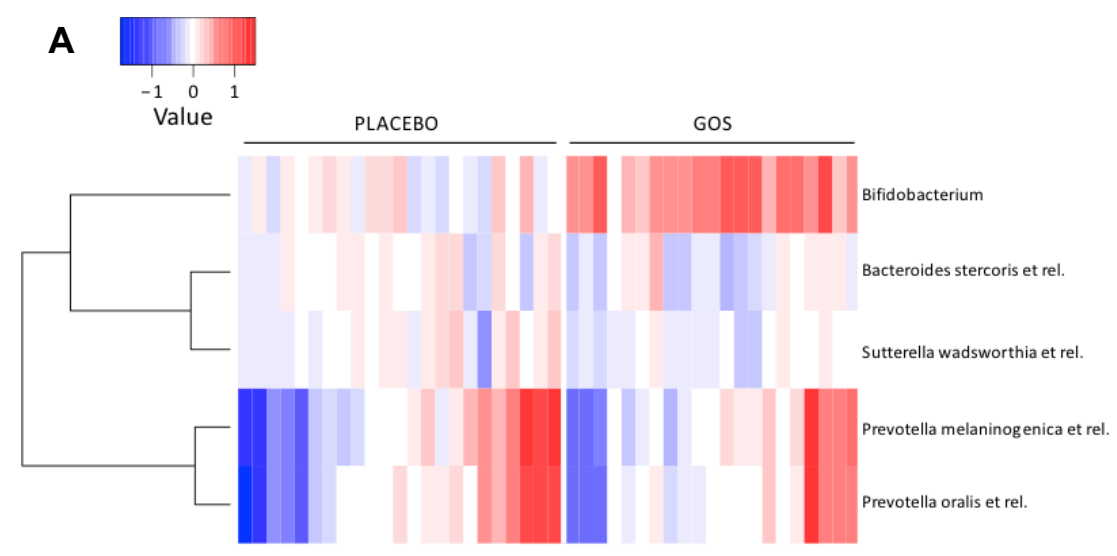

B
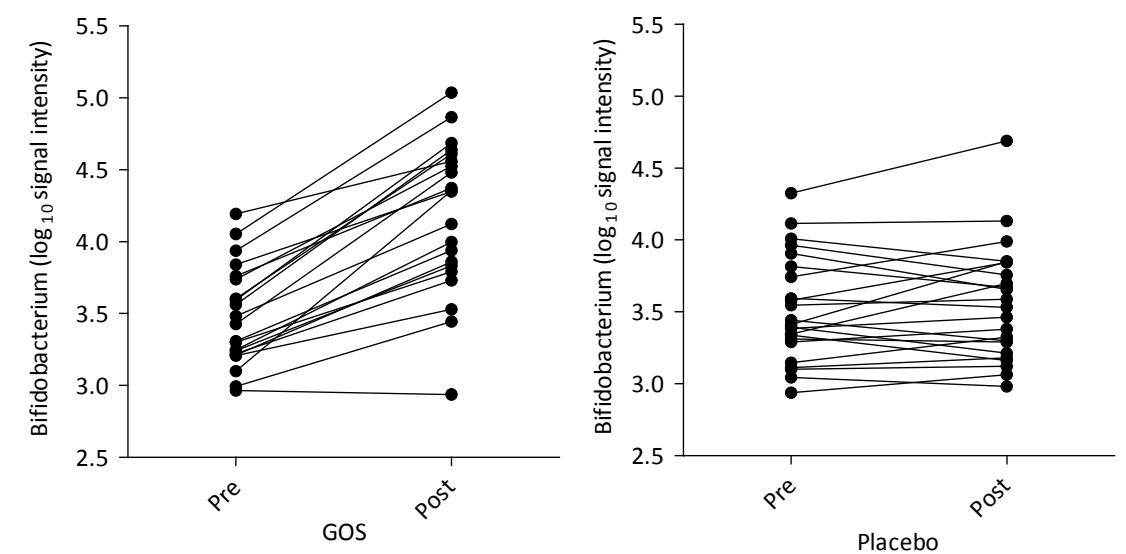

Heat map of the bacterial groups at genus-like taxonomic level, whose change in abundance was significantly different $(q<0.2)$ between $\mathrm{GOS}$ and placebo $(\mathbf{A})$. Bifidobacterium spp. relative abundance ( $\log 10$ signal intensity) as individual changes for the GOS group ( $n=21)$ and placebo group ( $n=23$ ), pre and post twelve-week supplementation with GOS or placebo (B). For HITChip analysis, log10-transformed signals were used as a proxy for bacterial logarithmic abundance. Color value shows log10 fold changes compared to baseline. Every column represents one individual participant. Differences between treatments were computed using a linear mixed model taking into account the effects of repeated measurements, treatment group, age and body mass index. Abbreviations: GOS, galacto-oligosaccharides. 
Figure 4.3. Insulin sensitivity before (pre) and after (post) GOS and placebo intervention
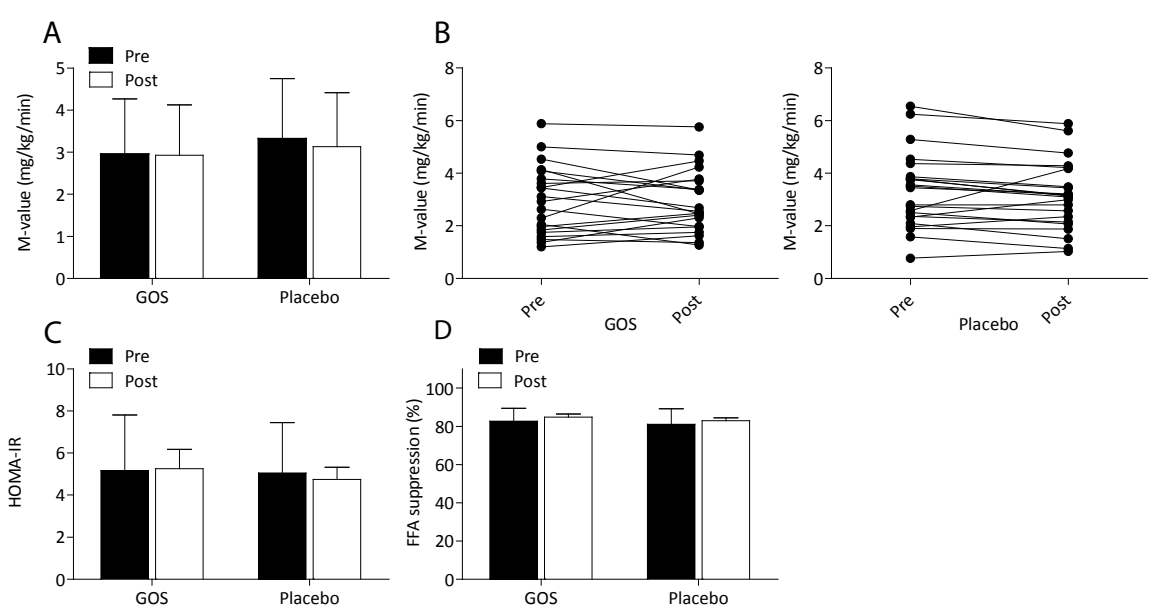

M-value given as means \pm SD (A) and as individual changes (B) for the GOS group ( $n=21)$ and placebo group ( $n=23$ ) pre and post twelve-week supplementation with GOS or placebo. HOMA-IR and insulin-stimulated (C) and FFA suppression (D) as a measure for adipose tissue insulin sensitivity pre and post twelve-week supplementation with GOS or placebo. Values are given as means \pm SD $(n=44)$ and data were analyzed using two-way repeated measures ANOVA, with time (pre, post) and intervention (GOS, placebo). No significant effects were detected. Abbreviations: GOS, galacto-oligosaccharides; FFA, free fatty acid; HOMA-IR, homeostatic model assessment of insulin resistance. 
Figure 4.4. Fecal and plasma concentrations of short-chain fatty acids before (pre) and after (post) GOS and placebo intervention

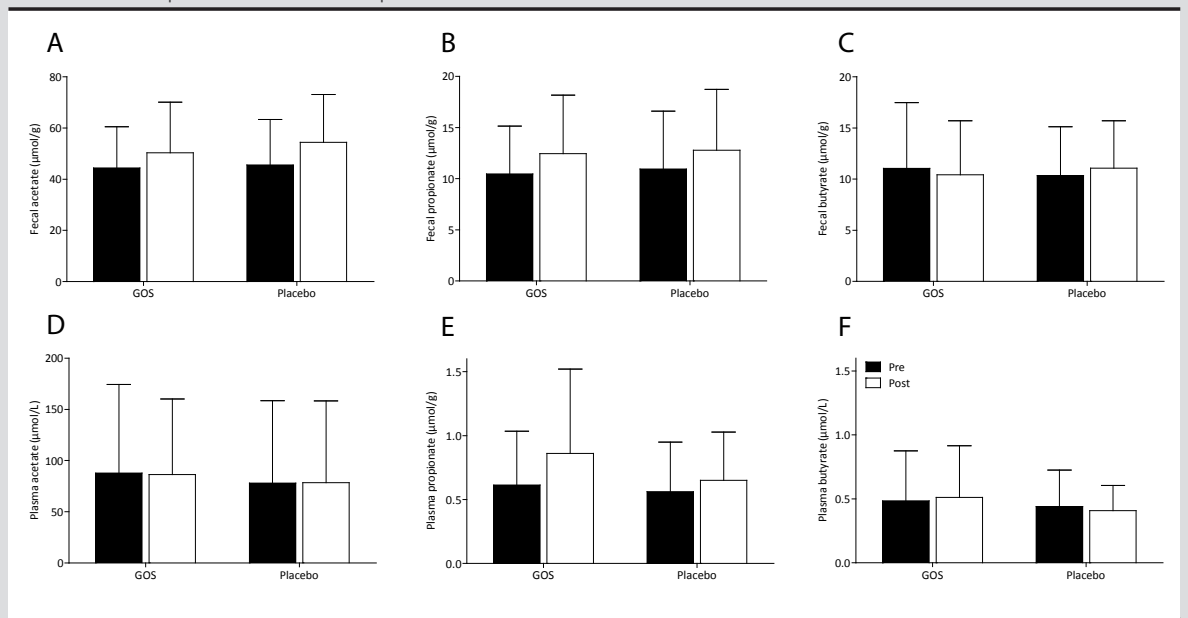

Fecal acetate (A), fecal propionate (B), and fecal butyrate (C) concentrations pre and post 12-week supplementation with GOS ( $n=21$ ) or placebo $(n=23)$. Plasma acetate (D), plasma propionate $(\mathbf{E})$ and plasma butyrate (F) concentrations pre and post twelve-week supplementation with GOS ( $n=21$ ) or placebo ( $n=23)$. Values are given as means \pm SD and data were analyzed using two-way repeated measures ANOVA, with time (pre, post) and intervention (GOS, placebo). No significant effects were determined. Abbreviations: GOS, galacto-oligosaccharides. 
Table 4.2. Body composition before (pre) and after (post) GOS and placebo intervention

\begin{tabular}{|c|c|c|c|c|}
\hline Variable & & GOS & Placebo & $P$-value \\
\hline \multirow[t]{2}{*}{ Body mass index $\left(\mathrm{kg} / \mathrm{m}^{2}\right)$} & pre & $33.3 \pm 3.7$ & $32.3 \pm 3.5$ & 0.68 \\
\hline & post & $33.7 \pm 3.7$ & $32.7 \pm 3.3$ & \\
\hline \multirow[t]{2}{*}{ Body weight (kg) } & pre & $98.4 \pm 11.9$ & $96.9 \pm 11.5$ & 0.69 \\
\hline & post & $99.5 \pm 12.3$ & $98.2 \pm 11.0$ & \\
\hline \multirow[t]{2}{*}{ Body fat (\%) } & pre & $36.8 \pm 7.8$ & $36.9 \pm 8.3$ & 0.17 \\
\hline & post & $37.1 \pm 7.6$ & $36.8 \pm 7.8$ & \\
\hline \multirow[t]{2}{*}{ Body fat (kg) } & pre & $36.3 \pm 8.9$ & $36.0 \pm 9.0$ & 0.23 \\
\hline & post & $37.1 \pm 8.9$ & $36.3 \pm 8.9$ & \\
\hline \multirow[t]{2}{*}{ Lean mass (kg) } & pre & $59.7 \pm 10.2$ & $58.9 \pm 9.5$ & 0.74 \\
\hline & post & $60.7 \pm 10.7$ & $59.7 \pm 9.1$ & \\
\hline \multirow[t]{2}{*}{ Visceral fat (g) } & pre & $932 \pm 353$ & $804 \pm 244$ & 0.89 \\
\hline & post & $928 \pm 343$ & $817 \pm 240$ & \\
\hline
\end{tabular}


Table 4.3. Plasma biochemistry before (pre) and after (post) GOS and placebo intervention

\begin{tabular}{|c|c|c|c|c|c|}
\hline Variable & & & GOS & Placebo & $P$-value \\
\hline \multicolumn{6}{|l|}{ Plasma metabolites } \\
\hline \multirow[t]{2}{*}{ Glucose (mmol/L) } & fasting & pre & $6.0 \pm 0.5$ & $5.8 \pm 0.4$ & 0.79 \\
\hline & & post & $6.0 \pm 0.5$ & $5.8 \pm 0.5$ & \\
\hline \multirow[t]{4}{*}{ TAG (mmol/L) } & fasting & pre & $1.28 \pm 0.42$ & $1.17 \pm 0.45$ & 0.54 \\
\hline & & post & $1.50 \pm 0.59$ & $1.31 \pm 0.51$ & \\
\hline & steady-state & pre & $1.19 \pm 0.54$ & $1.05 \pm 0.47$ & 0.71 \\
\hline & & post & $1.40 \pm 0.60$ & $1.25 \pm 0.59$ & \\
\hline \multirow[t]{4}{*}{ Free glycerol $(\mu \mathrm{mol} / \mathrm{L})$} & fasting & pre & $107 \pm 38$ & $99 \pm 22$ & 0.51 \\
\hline & & post & $100 \pm 28$ & $98 \pm 25$ & \\
\hline & steady-state & pre & $50.3 \pm 19$ & $48.6 \pm 15$ & 0.18 \\
\hline & & post & $46.2 \pm 13$ & $48.3 \pm 18$ & \\
\hline \multirow[t]{4}{*}{ FFAs $(\mu \mathrm{mol} / \mathrm{L})$} & fasting & pre & $910 \pm 274$ & $848 \pm 208$ & 0.33 \\
\hline & & post & $805 \pm 253$ & $809 \pm 214$ & \\
\hline & steady-state & pre & $119 \pm 59$ & $105 \pm 64$ & 0.71 \\
\hline & & post & $140 \pm 51$ & $122 \pm 64$ & \\
\hline \multicolumn{6}{|l|}{ Plasma hormones } \\
\hline \multirow[t]{2}{*}{ GLP-1 (pmol/L) } & fasting & pre & $11.3 \pm 2.6$ & $10.6 \pm 2.3$ & 0.69 \\
\hline & & post & $10.3 \pm 1.9$ & $9.9 \pm 2.4$ & \\
\hline \multirow[t]{2}{*}{$\operatorname{PYY}(p g / m L)$} & fasting & pre & $50.8 \pm 11.8$ & $47.5 \pm 9.6$ & 0.88 \\
\hline & & post & $53.4 \pm 12.2$ & $51.1 \pm 10.0$ & \\
\hline \multirow[t]{2}{*}{ Insulin (mU/L) } & fasting & pre & $20.7 \pm 8.8$ & $19.1 \pm 17.3$ & 0.71 \\
\hline & & post & $18.9 \pm 8.3$ & $18.3 \pm 10.1$ & \\
\hline \multirow[t]{2}{*}{ Leptin (ng/mL) } & fasting & pre & $31.9 \pm 19.7$ & $33.4 \pm 20.8$ & 0.36 \\
\hline & & post & $25.4 \pm 20.1$ & $28.8 \pm 21.0$ & \\
\hline \multicolumn{6}{|l|}{ Inflammatory markers } \\
\hline \multirow[t]{2}{*}{$\operatorname{LBP}(\mathrm{pg} / \mathrm{mL})$} & fasting & pre & $20.5 \pm 6.9$ & $26.4 \pm 19.3$ & 0.80 \\
\hline & & post & $21.0 \pm 7.6$ & $25.4 \pm 13.9$ & \\
\hline \multirow[t]{2}{*}{ IL-6 (pg/mL) } & fasting & pre & $0.82 \pm 0.30$ & $1.03 \pm 0.88$ & 0.59 \\
\hline & & post & $0.85 \pm 0.48$ & $0.99 \pm 0.48$ & \\
\hline \multirow[t]{2}{*}{ IL-8 (pg/mL) } & fasting & pre & $4.28 \pm 1.11$ & $3.89 \pm 1.07$ & 0.35 \\
\hline & & post & $4.32 \pm 1.30$ & $4.19 \pm 1.46$ & \\
\hline \multirow[t]{2}{*}{ TNF-a (pg/mL) } & fasting & pre & $2.35 \pm 0.63$ & $2.20 \pm 0.58$ & 0.38 \\
\hline & & post & $2.37 \pm 0.54$ & $2.32 \pm 0.49$ & \\
\hline
\end{tabular}

Values are given as means \pm SD and data were analyzed using two-way repeated measures ANOVA, with time (pre, post) and intervention (GOS $(n=21)$, placebo $(n=23))$. No significant effects. Abbreviations: FFAs, free fatty acids; GLP-1, glucagon-like peptide 1; GOS, galacto-oligosaccharides; LBP, lipopolysaccharide binding protein; PYY, peptide YY; TAG, plasma triacylglycerol, TNF-a, tumor necrosis factor alpha. 


\section{Energy expenditure, fat oxidation and carbohydrate oxidation}

GOS did not affect resting energy expenditure and insulin-mediated energy expenditure as compared to placebo treatment. In line, both resting as well as insulin-stimulated respiratory quotient and fasting and insulin-mediated fat and carbohydrate oxidation were not altered by GOS versus placebo (Table 4.4).

Table 4.4. Energy expenditure and substrate oxidation before (pre) and after (post) GOS and placebo intervention

\begin{tabular}{lllccc}
\hline Variable & & & GOS & Placebo & P-value \\
\hline Energy expenditure $(\mathrm{k} / \mathrm{min})$ & fasting & pre & $5.47 \pm 0.74$ & $5.30 \pm 0.63$ & 0.54 \\
& & post & $5.30 \pm 0.67$ & $5.32 \pm 0.63$ & \\
Energy expenditure $(\mathrm{k} / \mathrm{min})$ & steady-state & pre & $5.37 \pm 0.71$ & $5.20 \pm 0.62$ & 0.84 \\
& & post & $5.38 \pm 0.65$ & $5.22 \pm 0.13$ & \\
Fat oxidation (g/min) & fasting & pre & $0.083 \pm 0.019$ & $0.082 \pm 0.014$ & 0.24 \\
& & post & $0.076 \pm 0.020$ & $0.078 \pm 0.020$ & \\
Fat oxidation (g/min) & steady-state & pre & $0.054 \pm 0.022$ & $0.046 \pm 0.017$ & 0.17 \\
& & post & $0.047 \pm 0.021$ & $0.045 \pm 0.021$ & \\
Carbohydrate oxidation (g/min) & fasting & pre & $0.086 \pm 0.037$ & $0.072 \pm 0.033$ & 0.35 \\
& & post & $0.098 \pm 0.044$ & $0.084 \pm 0.043$ & \\
Carbohydrate oxidation (g/min) & steady-state & pre & $0.147 \pm 0.048$ & $0.158 \pm 0.045$ & 0.24 \\
& & post & $0.165 \pm 0.047$ & $0.163 \pm 0.052$ & \\
Respiratory quotient & fasting & pre & $0.79 \pm 0.03$ & $0.78 \pm 0.03$ & 0.97 \\
& & post & $0.80 \pm 0.04$ & $0.79 \pm 0.04$ & \\
Respiratory quotient & steady-state & pre & $0.84 \pm 0.04$ & $0.86 \pm 0.04$ & 0.20 \\
& & post & $0.86 \pm 0.05$ & $0.87 \pm 0.04$ & \\
\hline
\end{tabular}

Values are given as means \pm SD and data were analyzed using two-way repeated measures ANOVA, with time (pre, post) and intervention (GOS $(n=21)$, placebo $(n=23))$. No significant effects were detected. Abbreviations: GOS, galacto-oligosaccharides. 


\section{DISCUSSION}

In the present well-controlled human study, effects of GOS supplementation on measurements of microbiota composition and functionality (SCFAs), and detailed host metabolic phenotyping were combined. We demonstrated that twelve-week GOS supplementation markedly increased the abundance of fecal Bifidobacterium spp. without significant effects on overall microbial richness or diversity. Importantly, this specific bifidogenic effect did neither translate into alterations of fecal or plasma SCFA concentrations, nor into changes in systemic concentrations of gut-derived hormones, and systemic inflammatory markers. In addition, no significant alterations in peripheral and adipose tissue insulin sensitivity, body composition, energy and substrate metabolism, and circulating metabolites were found.

GOS supplementation (15 g/d) for twelve weeks increased the abundance of fecal Bifidobacterium spp., which is in accordance with previous studies, showing bifidogenic effects of different types of GOS in infants and adults. ${ }^{13,14,18,36-38}$ However, GOS supplementation did not result in any changes in host substrate and energy metabolism in the obese prediabetic participants. Dependent on substrate availability, fermentation by Bifidobacterium spp. mainly results in the production of lactate and acetate as end products. ${ }^{39}$ Indeed, fermentation of an identical type of GOS as used in the present study showed pronounced increases of acetate concentrations in the validated TNO in vitro model of the colon (TIM-2). ${ }^{17}$ Remarkably, we did not observe an increase in fecal or plasma acetate in vivo despite an average 5.0-fold increase in Bifidobacterium spp.. Importantly, we measured fecal and plasma SCFAs in fasted conditions and, therefore, cannot exclude that postprandial acetate concentration (i.e. 4 - 8 h after GOS ingestion) may have been higher in the GOS group.

Upon colonic arrival, GOS is quickly fermented in the cecum and the proximal part of the colon. ${ }^{40}$ Noteworthy, Boets et al. ${ }^{41}$ have indicated that a significant amount of colonically produced acetate is converted to other metabolites, in particular to butyrate, via microbial cross-feeding. Interestingly, GOS supplementation for 3 weeks increased the production of butyrate in adults over 50 years of age, but not of acetate, in a three-stage in vitro fermentation model using their fecal inocula. ${ }^{42}$ The microbial conversion of acetate to butyrate would result in a diminished availability of acetate to metabolically active organs, such as the liver, skeletal muscle, or adipose tissue. The fact that butyrate is a primary energy source for colonocytes and is mainly metabolized in the colon, ${ }^{15}$ might explain why no alterations in fecal and in fasting plasma butyrate concentrations were observed in the present study. In addition, we previously demonstrated that distal, but not proximal, colonic acetate infusions increased fat oxidation and increased circulating concentrations of acetate and PYY in overweight men. ${ }^{16}$ Distally applied acetate partly bypasses the liver 
via rectal veins, thereby directly reaching the systemic circulation, while more proximally administered acetate might be scavenged by the liver through portal drainage before it becomes systemically available. Thus, scavenging of acetate by the liver, the conversion of acetate into other metabolites, and the site of GOS fermentation may have led to an insufficient increase in systemic acetate concentrations to induce pronounced metabolic effects. Therefore, future research should focus on strategies to increase acetate concentrations specifically in the distal part of the colon in the longer-term. Such strategies could include the production of slow-fermentable acetogenic foods. ${ }^{43}$

The marked GOS-induced microbial changes without impact on energy and substrate metabolism, inflammatory profile and insulin sensitivity in the present study are in contrast to some ${ }^{13-15,44}$ but not all ${ }^{15,45}$ previous human studies using GOS or FOS. A crossover study by Vulevic et al. ${ }^{13}$ indicated that a twelve-week supplementation of a GOS mixture (5.5 g/d) decreased systemic inflammatory markers, fasting insulin, cholesterol and triglyceride concentrations in overweight participants. In addition, after GOS treatment, LPS-stimulated production of IL-6, IL-1 $\beta$, and TNF-a was lowered from isolated peripheral blood mononuclear cells in an ex vivo experiment. ${ }^{14}$ One of the explanations for the different metabolic outcomes between these studies and the present study might be the difference in population, which differed with respect to age and metabolic status. We included prediabetic overweight/obese men and postmenopausal women aged between 45 and $70 y$ in the present study, whereas Vulevic et al. ${ }^{13,14}$ investigated either a metabolically healthy elderly population, or overweight, but more insulin sensitive and younger adults. Ageing, obesity, and insulin resistance have been associated with alterations in gut microbiota composition, and microbial richness and diversity, as well as with a reduction of Bifidobacterium spp. specifically, and thus possibly with an altered metabolic response upon prebiotic treatment. ${ }^{46-49}$ In addition, recent studies suggest that microbiota from obese patients have a reduced fermentation capacity compared to microbiota from lean subjects. ${ }^{50-51}$ However, there remain inconsistencies, especially between human studies, regarding the aforementioned altered features of the microbiome. ${ }^{52}$ Furthermore, compared to animal experiments, the human microbiome is exposed to fundamentally different environmental factors that extend beyond the intervention alone such as heterogeneity of human (sub)population with regards to genetics, lifestyle, and diet. ${ }^{53}$ Overall, the metabolic imbalance and related progressed dysbiosis in our obese prediabetic population might have hampered improved metabolic effect via an altered microbial composition and activity. Therefore, the relevance of specific gut microbiota manipulation between different metabolic phenotypes, i.e. insulin resistant versus insulin sensitive humans, should be investigated in more detail in future studies.

When we compared the Bifidobacterium spp. of our study population with that of healthy, lean adults, the baseline abundance of Bifidobacterium spp. was markedly lower in our obese population, but reached slightly higher abundance after GOS intervention 
compared to this healthy, untreated, population (unpublished data, Laboratory of Microbiology, Wageningen UR, Wageningen, The Netherlands). In addition, we found overall small and inconsistent changes in the taxa Prevotella oralis et rel., Prevotella melaninogenica et rel., Bacteroides stercoris et rel., and Sutterella wadsworthia et rel. upon GOS ingestion compared to the placebo group. This in vivo data adds to previous in vitro studies showing that GOS feeding is highly specific at selectively enhancing bifidobacteria without consistently increasing or decreasing other specific bacterial lineages. ${ }^{19}$ It is possible that the high dosage of GOS in this study, $15 \mathrm{~g} / \mathrm{d}$ compared to other studies using $5.5 \mathrm{~g} / \mathrm{d},{ }^{13,14}$ resulted in more cross-feeding between specific members of the Bacteroidetes phylum and Bifidobacterium due to the increased availability of substrate. This provides an opportunity for other bacterial species to use GOS as source and to grow upon GOS supplementation. This is in line with a recent study indicating that supplementation of GOS $15 \mathrm{~g} / \mathrm{d}$ impacted at least eleven microbial genera, including Bifidobacterium. ${ }^{54}$ The small increase and particularly inconsistent pattern of increase, however, makes it highly unlikely that changes in these specific taxa explain the lack of effects on the metabolic outcomes in the present study.

In conclusion, we demonstrated in this well-controlled study, in which participants were phenotyped in detail, that twelve-week supplementation of GOS in prediabetic men and women increased the abundance of Bifidobacterium spp., yet no alterations in plasma or fecal SCFAs, peripheral insulin sensitivity, energy and substrate metabolism, and lowgrade inflammation were observed. Hence, the present study implies that a considerable diet-related increase in Bifidobacterium spp. does not significantly affect insulin sensitivity and parameters of the host substrate and energy metabolism immediately in a prediabetic population with overweight or obesity. 


\section{REFERENCES}

1. Delzenne NM, Cani PD, Everard A, et al. Gut microorganisms as promising targets for the management of type 2 diabetes. Diabetologia 2015;58:2206-2217.

2. Diamant M, Blaak E, De Vos W. Do nutrient-gut-microbiota interactions play a role in human obesity, insulin resistance and type 2 diabetes? Obesity Reviews 2011;12:272-281.

3. Clemente JC, Ursell LK, Parfrey LW, et al. The impact of the gut microbiota on human health: an integrative view. Cell 2012;148:1258-1270.

4. Robertson MD, Bickerton AS, Dennis AL, et al. Insulin-sensitizing effects of dietary resistant starch and effects on skeletal muscle and adipose tissue metabolism. The American journal of clinical nutrition 2005;82:559-567.

5. Vrieze A, Out C, Fuentes S, et al. Impact of oral vancomycin on gut microbiota, bile acid metabolism, and insulin sensitivity. Journal of hepatology 2014;60:824-831.

6. Vrieze A, Van Nood E, Holleman F, et al. Transfer of intestinal microbiota from lean donors increases insulin sensitivity in individuals with metabolic syndrome. Gastroenterology 2012;143:913-916. e7.

7. Hulston CJ, Churnside AA, Venables MC. Probiotic supplementation prevents high-fat, overfeeding-induced insulin resistance in human subjects. British Journal of Nutrition 2015;113:596-602.

8. Mikkelsen KH, Allin KH, Knop FK. Effect of antibiotics on gut microbiota, glucose metabolism and bodyweight regulation-a review of the literature. Diabetes, Obesity and Metabolism 2016;doi/10.1111/dom.12637.

9. Kellow NJ, Coughlan MT, Reid CM. Metabolic benefits of dietary prebiotics in human subjects: a systematic review of randomised controlled trials. British Journal of Nutrition 2014;111:1147-1161.

10. Ruan Y, Sun J, He J, et al. Effect of Probiotics on Glycemic Control: A Systematic Review and Meta-Analysis of Randomized, Controlled Trials. PloS one 2015;10:e0132121.

11. Roberfroid M, Gibson GR, Hoyles L, et al. Prebiotic effects: metabolic and health benefits. British Journal of Nutrition 2010;104:S1-S63.

12. Delzenne NM, Neyrinck AM, Cani PD. Gut microbiota and metabolic disorders: how prebiotic can work? British Journal of Nutrition 2013;109:S81-S85.

13. Vulevic J, Juric A, Tzortzis G, et al. A mixture of trans-galactooligosaccharides reduces markers of metabolic syndrome and modulates the fecal microbiota and immune function of overweight adults. The Journal of nutrition 2013;143:324-331.

14. Vulevic J, Drakoularakou A, Yaqoob P, et al. Modulation of the fecal microflora profile and immune function by a novel trans-galactooligosaccharide mixture (B-GOS) in healthy elderly volunteers. The American journal of clinical nutrition 2008;88:1438-1446.

15. Canfora EE, Jocken JW, Blaak EE. Short-chain fatty acids in control of body weight and insulin sensitivity. Nat Rev Endocrinol 2015;11:577-591.

16. van der Beek CM, Canfora EE, Lenaerts K, et al. Distal, not proximal, colonic acetate infusions promote fat oxidation and improve metabolic markers in overweight/obese men. Clinical Science 2016:CS20160263.

17. Maathuis AJ, van den Heuvel EG, Schoterman MH, et al. Galacto-oligosaccharides have prebiotic activity in a dynamic in vitro colon model using a 13C-labeling technique. The Journal of nutrition 2012;142:1205-1212. 
18. Holscher HD, Faust KL, Czerkies LA, et al. Effects of prebiotic-containing infant formula on gastrointestinal tolerance and fecal microbiota in a randomized controlled trial. Journal of Parenteral and Enteral Nutrition 2012;36:95S-105S.

19. Davis LM, Martínez I, Walter J, et al. Barcoded pyrosequencing reveals that consumption of galactooligosaccharides results in a highly specific bifidogenic response in humans. PLoS One 2011;6:e25200.

20. Pokusaeva K, Fitzgerald GF, van Sinderen D. Carbohydrate metabolism in Bifidobacteria. Genes \& nutrition 2011;6:285-306.

21. Wendel-Vos GW, Schuit AJ, Saris WH, et al. Reproducibility and relative validity of the short questionnaire to assess health-enhancing physical activity. Journal of clinical epidemiology 2003;56:1163-1169.

22. DeFronzo RA, Tobin JD, Andres R. Glucose clamp technique: a method for quantifying insulin secretion and resistance. American Journal of Physiology-Gastrointestinal and Liver Physiology 1979;237:G214-G223.

23. Jaquet $\mathrm{D}$, Gaboriau A, Czernichow $\mathrm{P}$, et al. Insulin resistance early in adulthood in subjects born with intrauterine growth retardation. J Clin Endocrinol Metab 2000;85:1401-6.

24. Weir JB. New methods for calculating metabolic rate with special reference to protein metabolism. J Physiol 1949;109:1-9.

25. Frayn KN. Calculation of substrate oxidation rates in vivo from gaseous exchange. J Appl Physiol 1983;55:628-34.

26. Orskov C, Rabenhoj L, Wettergren A, et al. Tissue and plasma concentrations of amidated and glycine-extended glucagon-like peptide I in humans. Diabetes 1994;43:535-9.

27. Froon AH, Dentener MA, Greve JWM, et al. Lipopolysaccharide toxicity-regulating proteins in bacteremia. Journal of Infectious Diseases 1995;171:1250-1257.

28. Garcia-Villalba R, Gimenez-Bastida JA, Garcia-Conesa MT, et al. Alternative method for gas chromatography-mass spectrometry analysis of short-chain fatty acids in faecal samples. Journal of separation science 2012;35:1906-13.

29. van Eijk HM, Bloemen JG, Dejong CH. Application of liquid chromatography-mass spectrometry to measure short chain fatty acids in blood. Journal of Chromatography B 2009;877:719-724.

30. Salonen A, Nikkilä J, Jalanka-Tuovinen J, et al. Comparative analysis of fecal DNA extraction methods with phylogenetic microarray: effective recovery of bacterial and archaeal DNA using mechanical cell lysis. Journal of microbiological methods 2010;81:127-134.

31. Rajilić-Stojanović M, Heilig HG, Molenaar D, et al. Development and application of the human intestinal tract chip, a phylogenetic microarray: analysis of universally conserved phylotypes in the abundant microbiota of young and elderly adults. Environmental microbiology 2009;11:1736-1751.

32. Lahti L, Elo LL, Aittokallio T, et al. Probabilistic analysis of probe reliability in differential gene expression studies with short oligonucleotide arrays. IEEE/ACM Transactions on Computational Biology and Bioinformatics (TCBB) 2011;8:217-225.

33. Bates D, Maechler M, Bolker B, et al. Package 'Ime4'. convergence 2015;12:1.

34. Bates $D$, Mächler $M$, Bolker $B$, et al. Fitting linear mixed-effects models using Ime4. arXiv preprint arXiv:1406.5823 2014.

35. Oksanen J, Blanchet FG, Kindt R, et al. Package 'vegan'. Community ecology package, version 2013;2. 
36. Depeint F, Tzortzis G, Vulevic J, et al. Prebiotic evaluation of a novel galactooligosaccharide mixture produced by the enzymatic activity of Bifidobacterium bifidum NCIMB 41171, in healthy humans: a randomized, double-blind, crossover, placebo-controlled intervention study. The American journal of clinical nutrition 2008;87:785-791.

37. Sierra C, Bernal M-J, Blasco J, et al. Prebiotic effect during the first year of life in healthy infants fed formula containing GOS as the only prebiotic: a multicentre, randomised, double-blind and placebo-controlled trial. European journal of nutrition 2015;54:89-99.

38. Vulevic J, Juric A, Walton GE, et al. Influence of galacto-oligosaccharide mixture (B-GOS) on gut microbiota, immune parameters and metabonomics in elderly persons. British Journal of Nutrition 2015;114:586-595.

39. Rossi M, Corradini C, Amaretti A, et al. Fermentation of fructooligosaccharides and inulin by bifidobacteria: a comparative study of pure and fecal cultures. Applied and environmental microbiology 2005;71:6150-6158.

40. Slavin J. Fiber and prebiotics: mechanisms and health benefits. Nutrients 2013;5:1417-1435.

41. Boets E, Gomand SV, Deroover $L$, et al. Systemic availability and metabolism of colonic-derived short-chain fatty acids in healthy subjects: a stable isotope study. The Journal of Physiology 2016.

42. Walton $\mathrm{GE}$, van den Heuvel EG, Kosters $\mathrm{MH}$, et al. A randomised crossover study investigating the effects of galacto-oligosaccharides on the faecal microbiota in men and women over 50 years of age. British Journal of Nutrition 2012;107:1466-1475.

43. Kaur A, Rose DJ, Rumpagaporn P, et al. In Vitro Batch Fecal Fermentation Comparison of Gas and Short-Chain Fatty Acid Production Using "Slowly Fermentable" Dietary Fibers. Journal of food science 2011;76:H137-H142.

44. Parnell JA, Reimer RA. Weight loss during oligofructose supplementation is associated with decreased ghrelin and increased peptide YY in overweight and obese adults. The American journal of clinical nutrition 2009;89:1751-1759.

45. Dewulf E, Cani P, Claus S, et al. Insight into the prebiotic concept: lessons from an exploratory, double blind intervention study with inulin-type fructans in obese women. Gut 2013;62:1112-1121.

46. O'Toole PW, Jeffery IB. Gut microbiota and aging. Science 2015;350:1214-1215.

47. Ley RE. Obesity and the human microbiome. Current opinion in gastroenterology 2010;26:511.

48. Tremaroli V, Bäckhed F. Functional interactions between the gut microbiota and host metabolism. Nature 2012;489:242-249.

49. Saraswati S, Sitaraman R. Aging and the human gut microbiota-from correlation to causality. Frontiers in microbiology 2013;5.

50. Ridaura VK, Faith Jj, Rey FE, et al. Gut microbiota from twins discordant for obesity modulate metabolism in mice. Science 2013;341:1241214.

51. Khan MT, Nieuwdorp M, Bäckhed F. Microbial modulation of insulin sensitivity. Cell metabolism 2014;20:753-760.

52. Walters WA, Xu Z, Knight R. Meta-analyses of human gut microbes associated with obesity and IBD. FEBS letters 2014;588:4223-4233.

53. Hermes G, Zoetendal E, Smidt H. Molecular ecological tools to decipher the role of our microbial mass in obesity. Beneficial microbes 2014;6:61-81.

54. Azcarate-Peril MA, Ritter AJ, Savaiano D, et al. Impact of short-chain galactooligosaccharides on the gut microbiome of lactose-intolerant individuals. Proceedings of the National Academy of Sciences 2017:201606722. 


\section{SUPPLEMENTAL FILES}

Supplemental Figure 4.1. Flow diagram of human participants included in this study

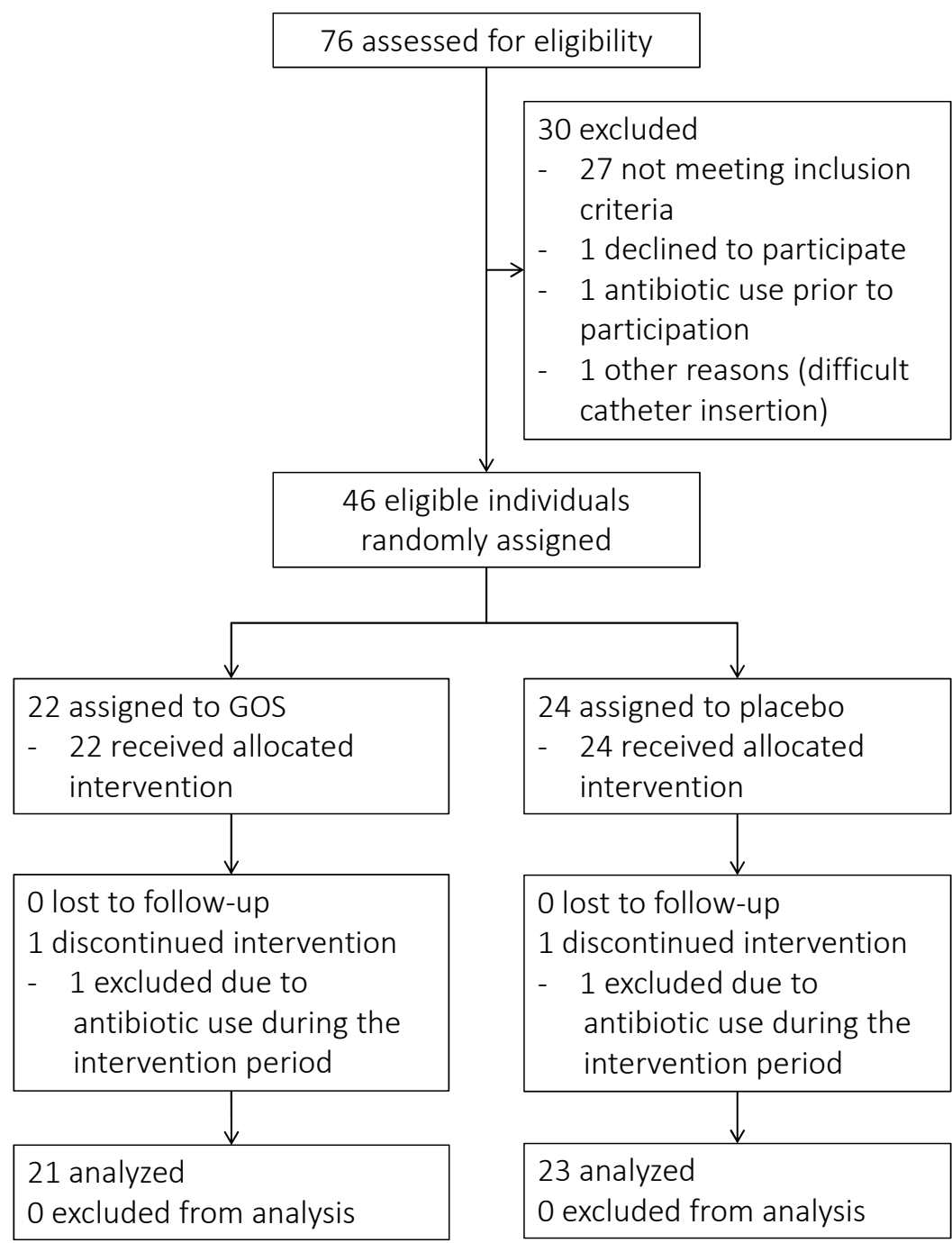


Supplemental Figure 4.2. Gut microbiota composition before (pre) and after (post) GOS and placebo intervention

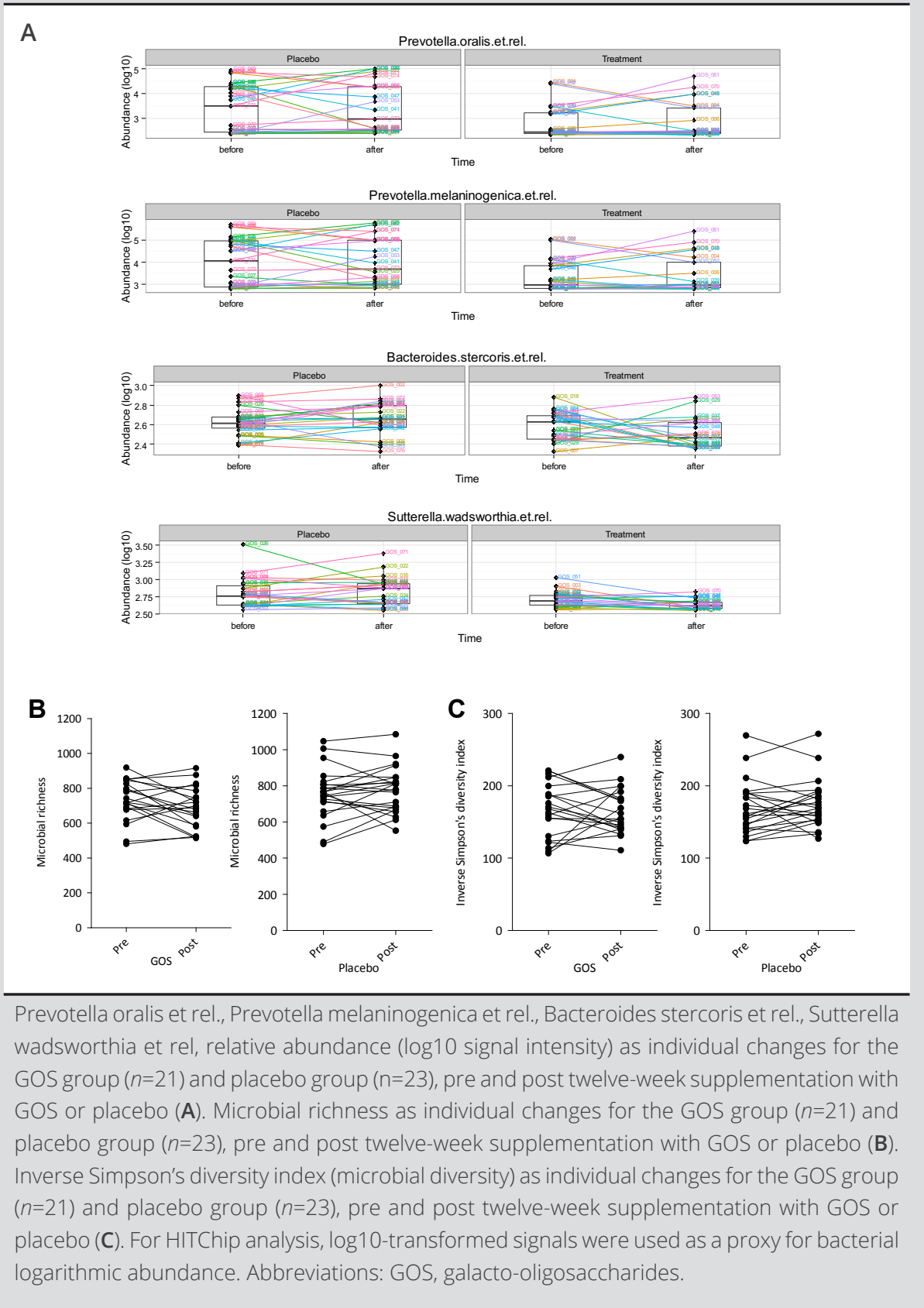


Supplemental Table 4.1. Food intake and physical activity scores (SQUASH) pre and post GOS and placebo intervention

\begin{tabular}{lccc}
\hline & GOS & & \\
\hline Time & Baseline & Week 6 & Week 12 \\
\hline Energy intake (kcal) & $2456 \pm 758$ & $2470 \pm 665$ & $2226 \pm 504$ \\
Fat intake (g) & $101 \pm 44$ & $83 \pm 29$ & $79 \pm 32$ \\
Carbohydrate intake (g) & $256 \pm 92$ & $262 \pm 89$ & $247 \pm 66$ \\
Protein intake (g) & $104 \pm 39$ & $112 \pm 26$ & $105 \pm 18$ \\
Dietary fiber intake & $20.9 \pm 5.1$ & $19.8 \pm 7.0$ & $18.5 \pm 4.9$ \\
(without GOS) (g) & & & 18 \\
\hline Meeting Dutch guidelines & 18 & 180 & $1342 \pm 1034$ \\
for physical activity (n) & $1009 \pm 787$ & $1188 \pm 867$ & \\
\hline Light physical & & & \\
activity (min/week) & $657 \pm 533$ & $581 \pm 336$ & \\
Moderate physical & & & \\
activity (min/week) & $53 \pm 94$ & $52 \pm 137$ & \\
Vigorous physical & & & \\
activity (min/week) & & & \\
\hline
\end{tabular}

Values are given as means \pm SD and data were analyzed using a two-way repeated measures ANOVA, with time (pre, post) and intervention (GOS $(n=21)$, placebo $(n=23)$ ). Abbreviations: GOS, galacto-oligosaccharides. 


\begin{tabular}{cccc}
\hline \multicolumn{2}{c}{ Placebo } & & $P$-value \\
\hline Baseline & Week 6 & Week 12 & \\
\hline $2235 \pm 636$ & $2282 \pm 539$ & $2198 \pm 628$ & 0.513 \\
$87 \pm 34$ & $78 \pm 29$ & $78 \pm 34$ & 0.456 \\
$242 \pm 73$ & $242 \pm 73$ & $243 \pm 75$ & 0.873 \\
$88 \pm 21$ & $97 \pm 21$ & $98 \pm 19$ & 0.589 \\
$21.6 \pm 5.8$ & $21.5 \pm 5.9$ & $19.4 \pm 5.5$ & 0.912 \\
& & & 0.740 \\
17 & 19 & 18 & 0.310 \\
$703 \pm 593$ & $1090 \pm 886$ & $1352 \pm 1142$ & 0.114 \\
$63 \pm 129$ & $912 \pm 814$ & $743 \pm 586$ & 0.223 \\
\hline
\end{tabular}




$$
5
$$


This chapter is embargoed at request

\section{CHAPTER 5}

Expression of short-chain fatty acid receptors GPR41, GPR43, and GPR109A is higher in the human ileum and proximal colon compared with the distal colon

Christina M. van der Beek, Mark van Avesaat, Nikkie van der Wielen, Anna M. Kip, FreddyJ. Troost, Ad A. M. Masclee, Cornelis H. C. Dejong, Kaatje Lenaerts 


$$
6
$$




\section{CHAPTER 6}

\section{Hepatic uptake of rectally administered butyrate prevents an increase in systemic butyrate concentrations in humans}

Christina M. van der Beek*, Johanne G. Bloemen*, Maartje A. van den Broek, Kaatje Lenaerts, Koen Venema, Wim A. Buurman, Cornelis H. C. Dejong

*Both authors contributed equally

Journal of Nutrition. 2015;145(9):2019-24 


\section{ABSTRACT}

Background: Short-chain fatty acids (SCFAs), fermentation products of undigested fibers, are considered beneficial for colonic health. High plasma concentrations are potentially harmful, and therefore information about systemic SCFA clearance is needed before therapeutic use of prebiotics or colonic SCFA administration.

Objective: The aim of this study was to investigate the effect of rectal butyrate administration on plasma SCFA concentrations and inter-organ exchange.

Methods: Twelve patients (7 men, age $66.4 \pm 2.0$ y, BMI $24.5 \pm 1.4 \mathrm{~kg} / \mathrm{m}^{2}$ ) undergoing upper abdominal surgery were included in this randomized placebo-controlled trial. During surgery, one group received a butyrate enema $100 \mathrm{mmol} / \mathrm{L}$ sodium butyrate, 60 $\mathrm{mL}, n=7$ ), and the other group a placebo enema (140 mmol/L 0.9\% NaCl, $60 \mathrm{~mL}, n=5$ ). Before and 5, 15, and 30 min after administration, plasma samples were taken from the radial artery, hepatic vein and portal vein. SCFA concentrations were analyzed and fluxes from portal drained viscera, liver and splanchnic area were calculated, and used for the calculation of the incremental area under the curve (iAUC) over a 30-min period.

Results: Rectal butyrate administration led to higher portal venous butyrate concentrations at $5 \mathrm{~min}$ compared with placebo $(92.2 \pm 27.0 \mu \mathrm{mol} / \mathrm{L}$ vs $14.3 \pm 3.4 \mu \mathrm{mol} / \mathrm{L}$, respectively; $P<0.01)$. In addition, arterial butyrate concentrations were higher at 5 min compared with placebo $(7.2 \pm 1.8 \mu \mathrm{mol} / \mathrm{L}$ vs $0.6 \pm 0.1 \mu \mathrm{mol} / \mathrm{L}$, respectively; $P<0.01)$. iAUC of portal venous butyrate concentrations was significantly higher after butyrate than after placebo administration (1083.3 $\pm 447.4 \mu \mathrm{mol} / \mathrm{L} \cdot 0.5 \mathrm{~h}$ vs $-68.5 \pm 58.5 \mu \mathrm{mol} / \mathrm{L} \cdot 0.5 \mathrm{~h}$, respectively; $P<0.01$ ) and iAUC of arterial butyrate concentrations were also significantly higher after butyrate than after placebo administration (141.6 $\pm 48.2 \mu \mathrm{mol} / \mathrm{L} \cdot 0.5 \mathrm{~h}$ vs 3.2 $\pm 6.4 \mu \mathrm{mol} / \mathrm{L} \cdot 0.5 \mathrm{~h}$, respectively; $P<0.05)$. The iAUCs of gut release $(282.8 \pm 133.8 \mu \mathrm{mol} /$ $\mathrm{kg}$ body weight (BW) $0.5 \mathrm{~h}$ ) and liver uptake (-293.7 $\pm 136.0 \mu \mathrm{mol} / \mathrm{kg} \mathrm{BW} \cdot 0.5 \mathrm{~h}$ ) of butyrate were greater in the butyrate-treated group compared with placebo $(-16.6 \pm 13.4 \mu \mathrm{mol} / \mathrm{kg}$ BW·0.5 h for gut release and $16.0 \pm 13.8 \mu \mathrm{mol} / \mathrm{kg}$ BW·0.5 h for liver uptake, respectively; $P=0.01$ and $P<0.05$, respectively). As a result, splanchnic butyrate release did not differ between groups. Fractional colonic absorption of butyrate was $18.3 \%$ in the 30 -min period after butyrate administration. Butyrate administration did not affect acetate or propionate exchange.

Conclusions: After receiving a butyrate enema, gut release and hepatic uptake of butyrate occurred to a similar extent, preventing splanchnic butyrate release in patients undergoing upper abdominal surgery. These observations imply that therapeutic colonic SCFA administration at this dose is safe. 


\section{INTRODUCTION}

Prebiotics currently receive considerable attention because of their potential benefits for gut health. Prebiotics are non-digestible food ingredients, mostly carbohydrates, which are fermented by colonic bacteria resulting in the production of short-chain fatty acids (SCFAs). ${ }^{1}$ The predominant SCFAs produced are acetate, propionate and butyrate. SCFAs are rapidly taken up by the epithelial cells lining the gut and act locally as an important energy source for colonocytes. Furthermore, they protect the colonocytes against mechanical, chemical, and microbial damage., ${ }^{2,3}$ Especially butyrate is important in cellular homeostasis of the intestine and influences various processes. ${ }^{4,5}$ Butyrate is known to stimulate cell proliferation in normal colonocytes whereas it inhibits cell proliferation in cancer cell lines in vitro. ${ }^{6}$ In addition, butyrate alters the expression of signaling genes in the intestinal mucosa thereby positively affecting mucosal immunity. ${ }^{7,8}$ The effects of prebiotics or colonic SCFA administration on the intestinal mucosa have been investigated in patients with a variety of intestinal diseases, such as ulcerative colitis, Crohn's disease, pouchitis, diversion colitis, radiation proctitis, and irritable bowel syndrome. ${ }^{9-12}$ Although results are not consistent, major evidence supports the concept that intestinal SCFA supplementation improves colonic disease. ${ }^{13-18}$

Hence, the colonic effects of butyrate and other SCFAs are widely studied. Knowledge about the exchange of SCFAs across the gut, liver, and splanchnic area in humans is however scarce. In sudden death victims, portal butyrate concentrations ranged from 14 - 64 $\mu \mathrm{mol} / \mathrm{L}$. In the hepatic vein butyrate concentrations in all victims were lower and ranged from 2 - $32 \mu \mathrm{mol} / \mathrm{L} .{ }^{19}$ It is currently unknown whether these results are representative for living humans. Recently, our group confirmed that the intestine is the main SCFA releasing organ in humans, and that the liver is capable of taking up all propionate and butyrate released from the gut, even in a situation of chronic liver disease..$^{20,21}$ This physiological information is highly relevant because prebiotics are currently studied as therapeutic agents for certain intestinal diseases. Importantly, high systemic concentrations of SCFAs could be harmful; high intravenous butyrate supplementation can cause side effects such as hypokalemia and nausea. ${ }^{22,23}$ Exact concentrations at which systemic butyrate causes these side effects are unknown. Because butyrate has the highest potential for curing or treating colonic diseases, information about trafficking and hepatic clearance of this SCFA is of utmost importance to assure that administration of prebiotics or SCFAs can be performed in a secure manner. Therefore, the aim of this study was to investigate the effect of a clinically relevant dose of butyrate administered via an enema on systemic SCFA concentrations and on the inter-organ exchange across the splanchnic organs (gut and liver) in humans. 


\section{METHODS}

\section{Patients}

Due to the anatomical location of the hepatic and portal vein, patients were studied during upper abdominal surgery, either open liver resection or pancreatico-duodenectomy, between June and November 2009 at Maastricht University Medical Centre + (MUMC+). Patients with known parenchymal liver disease, presence of ileostomy or colostomy, and patients with inflammatory bowel disease were excluded from the study. The patients were on a stable, Western diet and did not receive antibiotics, prebiotics or probiotics 2 months prior to surgery. Oral intake except water was ceased at $20.00 \mathrm{~h}$ the day before surgery and all patients were transported to the operating theatre at approximately $8.00 \mathrm{~h}$ the next day. All patients received a single intravenous dose of 2,200 mg amoxicillin/clavulanic acid as antibiotic prophylaxis, immediately preoperatively. The study was approved by the Medical Ethical Committee of MUMC+ and conducted in accordance with the Declaration of Helsinki (revised version, October 2008, Seoul). Written informed consent was obtained from all patients.

\section{Study design}

The study was a blinded randomized placebo-controlled clinical trial. We anticipated that the placebo group would have less inter-individual variation in systemic SCFA concentrations after enema administration and therefore patients were randomized in a 1:2 ratio to receive placebo or butyrate. A power calculation determined a sample size of 15 patients, hence, 5 patients were randomized to receive a placebo enema, and 10 patients to a butyrate enema. The pharmacist performed simple randomization and the enemas were numbered 1 to 15. After the experiment was completed and the samples were analyzed, the randomization key was given to the researcher. The enemas were kindly prepared and supplied by the pharmacist of MUMC+ and contained either a sodium butyrate solution (60 mL, $100 \mathrm{mmol} / \mathrm{L}$ ) or a placebo solution (60 mL, $140 \mathrm{mmol} / \mathrm{L}$ 0.9\% NaCl), both with a neutral $\mathrm{pH}$. Both patients and investigators analyzing the samples and managing the data were blinded for the type of enema. The person administering the enema could not be blinded due to the odor of the enema. The same type of enema has also been used before in other studies at our institute by Hamer et al. ${ }^{18}$ and was proven to be safe. The primary outcome measure was plasma concentration and flux of butyrate, secondary outcomes were plasma concentrations and fluxes of acetate and propionate.

Anesthetic management was performed according to institutional routines as has been described before. ${ }^{24}$ After anesthesia was induced, a silicon Foley balloon catheter (30 Ch.) was placed in the rectum and the balloon was filled with $10 \mathrm{~mL} 0.9 \% \mathrm{NaCl}$ to prevent leakage after enema administration. During laparotomy, when exposure of the portal vein and hepatic veins was adequate, mostly within one hour following laparotomy and before 
organ transection, baseline ( $\mathrm{t}=0 \mathrm{~min}$ ) blood samples were drawn from the portal vein and a hepatic vein by direct puncture, simultaneously with arterial blood sampling, as described before by our group. ${ }^{21,25}$ Directly thereafter, a $60 \mathrm{~mL}$ enema containing either the sodium butyrate solution or the placebo solution, was administered through the rectal catheter. Subsequently, blood was drawn from the portal vein, a hepatic vein and the radial artery at three time points, namely 5, 15, and 30 min after administration of the enema. Blood was collected in EDTA vacuum tubes (BD Vacutainer, Franklin Lakes, NJ) and immediately placed on ice. Blood was centrifuged at $3,500 \mathrm{~g}, 4^{\circ} \mathrm{C}$ and plasma was stored at $-80^{\circ} \mathrm{C}$ until analysis.

\section{Sample preparation and liquid chromatography-mass spectrometry (LC-MS) analysis}

Deproteinization and subsequent preparation of plasma samples for analysis of SCFAs was performed as reported before. ${ }^{26}$ Analysis was performed using a liquid chromatography system combined with mass spectrometry. The detection limits for acetate, propionate, and butyrate of this method were $0.1,0.05$, and $0.05 \mu \mathrm{mol} / \mathrm{L}$, respectively. The coefficients of variance were $4.2 \%, 9.8 \%$, and $5.1 \%$ for acetate, propionate, and butyrate in human plasma, respectively.

\section{Flux calculations}

Arteriovenous differences $(\triangle A V)$ and net organ fluxes (flow $\cdot \triangle A V$ ) were calculated across the portal drained viscera (PDV), liver, and the splanchnic area, as described previously. ${ }^{21}$ Positive fluxes indicate net release, while negative fluxes indicate net uptake. Calculated fluxes are a measure of net exchange across an organ; they indicate whether a substance is taken up or released by that organ.

For plasma flow, data were used from a similar study conducted previously, as it was logistically impractical, and considered unethical to repeatedly measure flow at the indicated time points in the present study. ${ }^{25}$ Therefore, splanchnic plasma flow was calculated as portal flow (320 mL/min) plus hepatic artery flow $(110 \mathrm{~mL} / \mathrm{min})$.

\section{Statistics}

Data are presented as mean \pm SEM. Normality of the data was checked using a Kolmogorov-Smirnov test. In case the data did not meet normality assumptions, log transformation was used. Homogeneity of variance was estimated by Levene's Test for Equality of Variances. Differences between butyrate $(n=7)$ and placebo $(n=5)$ treatment were tested using incremental area under the curve (iAUC) and two-way repeated measures ANOVA for treatment effect with Bonferroni post-hoc correction to compare effects at specific time points. For within-group comparisons, a Friedman test for repeated measures followed by Dunn's post-hoc test was used. Baseline concentrations for butyrate and placebo and differences in iAUC after treatment were compared using an unpaired $t$-test. A two-sided $P$-value of $<0.05$ was considered statistically significant. Statistical analyses were performed using SPSS 22.0 for Windows. 


\section{RESULTS}

\section{Patients}

Fifteen patients were included in the study. Three patients, all randomized to the butyrate enema group, were excluded from final analysis. This was due to technical failures in two patients, namely malfunction of the arterial catheter during the experiment in one patient and accidentally swapping of the rectal catheter with the bladder catheter by theatre staff in the other patient. The third patient was excluded for medical reasons since the liver was more severely damaged as expected before surgery. As a result, in total 12 patients, 7 in the butyrate-treated group and 5 in the placebo group, were included in the final analysis (Table 6.1 and Figure 6.1). Baseline characteristics, including plasma concentrations, did not differ between the two groups. (Table 6.2). No adverse effects were observed.

Table 6.1. Baseline characteristics

\begin{tabular}{lll}
\hline Variable & Placebo $(n=5)$ & Butyrate $(n=7)$ \\
\hline Male $(n)$ & 2 & 5 \\
Age $(\mathrm{y})$ & $69 \pm 3.7$ & $65 \pm 2.3$ \\
BMI $\left(\mathrm{kg} / \mathrm{m}^{2}\right)$ & $24.5 \pm 1.8$ & $24.5 \pm 2.1$ \\
Indication $(n)$ & & \\
$\quad$ Colorectal liver metastases & 0 & 6 \\
$\quad$ Pancreatic pathology & 4 & 1 \\
$\quad$ Biliary pathology & 1 & 0 \\
\hline
\end{tabular}

There were no statistically significant differences between the two groups, $n=12$.

Table 6.2. Plasma SCFA concentrations at baseline in human subjects receiving a butyrate or placebo enema during upper abdominal surgery

\begin{tabular}{lllllll}
\hline & Arterial & & Portal vein & \multicolumn{2}{l}{ Hepatic vein } \\
\hline & Butyrate & Placebo & Butyrate & Placebo & Butyrate & Placebo \\
\hline Acetate $(\mu \mathrm{mol} / \mathrm{L})$ & $126 \pm 23$ & $86 \pm 20$ & $236 \pm 69$ & $182 \pm 47$ & $177 \pm 61$ & $71 \pm 9$ \\
$\begin{array}{l}\text { Propionate } \\
(\mu \mathrm{mol} / \mathrm{L})\end{array}$ & $1.6 \pm 1.0$ & $0.9 \pm 0.1$ & $18.1 \pm 7.3$ & $30.2 \pm 11.4$ & $3.6 \pm 1.0$ & $0.8 \pm 0.1$ \\
Butyrate $(\mu \mathrm{mol} / \mathrm{L})$ & $1.1 \pm 0.4$ & $0.7 \pm 0.2$ & $26.2 \pm 15.8$ & $15.1 \pm 3.5$ & $3.8 \pm 1.6$ & $0.5 \pm 0.1$ \\
\hline $\begin{array}{l}\text { Values are means } \pm \mathrm{SEM}, n=5 \text { (placebo) or } n=7 \text { (butyrate). There were no differences between } \\
\text { the groups. }\end{array}$
\end{tabular}


Figure 6.1. Flow diagram of human subjects receiving a butyrate or placebo enema during upper abdominal surgery

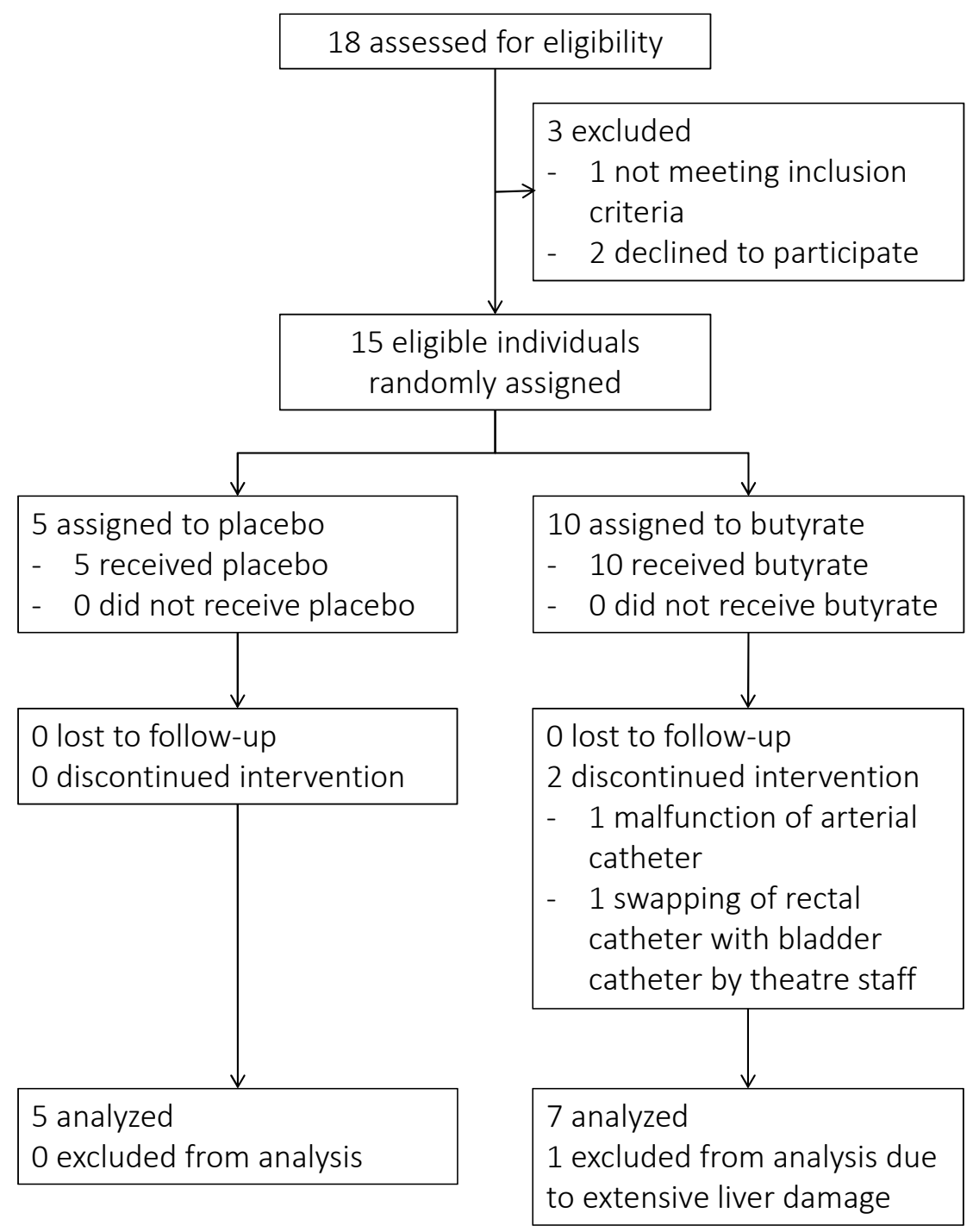




\section{Butyrate concentrations}

Baseline portal venous butyrate concentrations were not different between the two groups. Rectal butyrate administration led to a rapid increase in portal venous butyrate concentrations 5 min after administration compared with baseline concentrations $(P<0.01)$. Portal venous butyrate concentrations were not significantly different from baseline after placebo administration (Figure 6.2A). As a result, 5 min after enema administration, portal venous butyrate concentrations were significantly higher in the butyrate-treated group compared with placebo $(92.2 \pm 27.0 \mu \mathrm{mol} / \mathrm{L}$ vs $14.3 \pm 3.4 \mu \mathrm{mol} / \mathrm{L}, P<0.01)$. This was also reflected by a significantly higher iAUC of portal venous butyrate in the butyrate-treated group when compared with placebo $(P<0.01$, Figure 6.2D).

Figure 6.2. Plasma butyrate concentrations
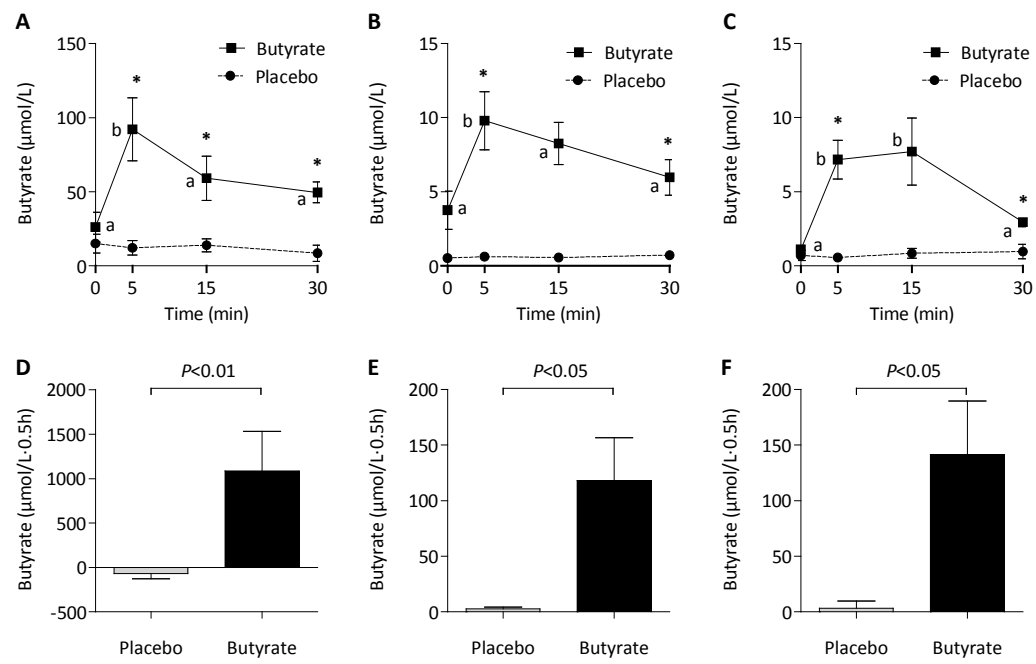

Portal venous ( $\mathbf{A}$ and $\mathbf{D}$ ), hepatic venous (B and $\mathbf{E})$, and arterial ( $\mathbf{C}$ and $\mathbf{F}$ ) butyrate concentrations and corresponding incremental area under the curve (iAUC) following placebo and butyrate enema. A: Overall intervention effect after butyrate enema, $P<0.05$, B: Overall intervention effect after butyrate enema, $P<0.01$, C: Overall intervention effect after butyrate enema, $P<0.05$. *Different from placebo at respective time point, $P<0.05$. Labeled means $(a, b)$ within a group without a common letter differ, $P<0.05$. Values are means $\pm \mathrm{SEM}, n=5$ (placebo) or $n=7$ (butyrate).

At baseline, butyrate concentrations in the hepatic vein tended to be higher in the butyrate-treated group compared with placebo $(P=0.08)$. Rectal butyrate administration led to a rapid increase in butyrate concentrations in the hepatic vein 5 min after 
administration compared with concentrations at baseline $(P<0.05)$. As expected, placebo administration did not affect hepatic venous butyrate concentrations (Figure 6.2B). Five min after enema administration, butyrate concentrations in the hepatic vein were significantly higher in the butyrate-treated group compared with placebo $(9.8 \pm 2.5 \mu \mathrm{mol} / \mathrm{L}$ vs 0.6 $\pm 0.2 \mu \mathrm{mol} / \mathrm{L}, P<0.05)$. This was also reflected by significantly higher butyrate concentrations in the hepatic vein, expressed as iAUC, in the butyrate-treated group when compared with placebo $(P<0.05$, Figure 6.2E). Butyrate concentrations in the hepatic vein showed a similar pattern as observed for the portal vein (Figure 6.2A and 6.2B).

Baseline arterial butyrate concentrations were not different between the two groups. Arterial butyrate concentrations were significantly increased after the butyrate enema at 5 and 15 min after administration compared with baseline $(P<0.05)$, and were not altered after placebo enema (Figure 6.2C), which was also reflected by significantly higher arterial butyrate concentrations, expressed as iAUC, in the butyrate-treated group when compared with placebo ( $P<0.05$, Figure 6.2F). Five min after instillation, arterial concentrations were significantly higher in the butyrate-treated group compared with placebo (7.2 \pm 1.8 $\mu \mathrm{mol} / \mathrm{L}$ vs $0.6 \pm 0.1 \mu \mathrm{mol} / \mathrm{L}, P<0.05)$.

\section{Butyrate exchange}

At baseline, net butyrate production by the gut (net release into the portal vein), liver metabolism, and splanchnic release of butyrate did not significantly differ between the butyrate-treated and placebo group (Figure 6.3A, 6.3B, and 6.3C).

Rectal butyrate administration resulted in a significant increase in butyrate gut release 5 min after administration compared with baseline $(P<0.01)$, whereas it did not change in the placebo group (Figure 6.3A). The peak gut release of butyrate tended to be higher in the butyrate-treated group compared with the placebo group at $5 \mathrm{~min}(P=0.07)$. Thirty min after administration, butyrate gut release was still higher in the butyrate-treated group compared with placebo (12.6 $\left.\pm 2.4 \mu \mathrm{mol} \cdot \mathrm{kg} \mathrm{BW}^{-1} \cdot \mathrm{h}^{-1} \mathrm{vs} 2.3 \pm 1.1 \mu \mathrm{mol} \cdot \mathrm{kg} \mathrm{BW}^{-1} \cdot \mathrm{h}^{-1}, P<0.05\right)$. In line, butyrate gut release, expressed as IAUC, was significantly higher in the butyratetreated group when compared with placebo ( $P=0.01$, Figure 6.3D).

Rectal butyrate administration resulted in a significant increase in liver uptake of butyrate, reflected by a negative flux, 5 min after administration compared with baseline $(P<0.05)$, whereas it did not change in the placebo group (Figure 6.3B). The peak liver flux of butyrate tended to be lower in the butyrate-treated group compared with the placebo group at $5 \mathrm{~min}\left(-22.2 \pm 11.0 \mu \mathrm{mol} \cdot \mathrm{kg} \mathrm{BW}^{-1} \cdot \mathrm{h}^{-1} \mathrm{vs}-4.0 \pm 0.1 \mu \mathrm{mol} \cdot \mathrm{kg} \mathrm{BW}^{-1} \cdot \mathrm{h}^{-1} ; P=0.09\right.$; Figure $6.3 \mathrm{~B})$, indicating a tendency to higher net liver uptake at this time point. In line, the iAUC of hepatic uptake of butyrate revealed a significant higher uptake in the butyrate-treated group when compared with the placebo group ( $P<0.05$, Figure 6.3E). 
Figure 6.3. Butyrate exchange across gut, liver and splanchnic area
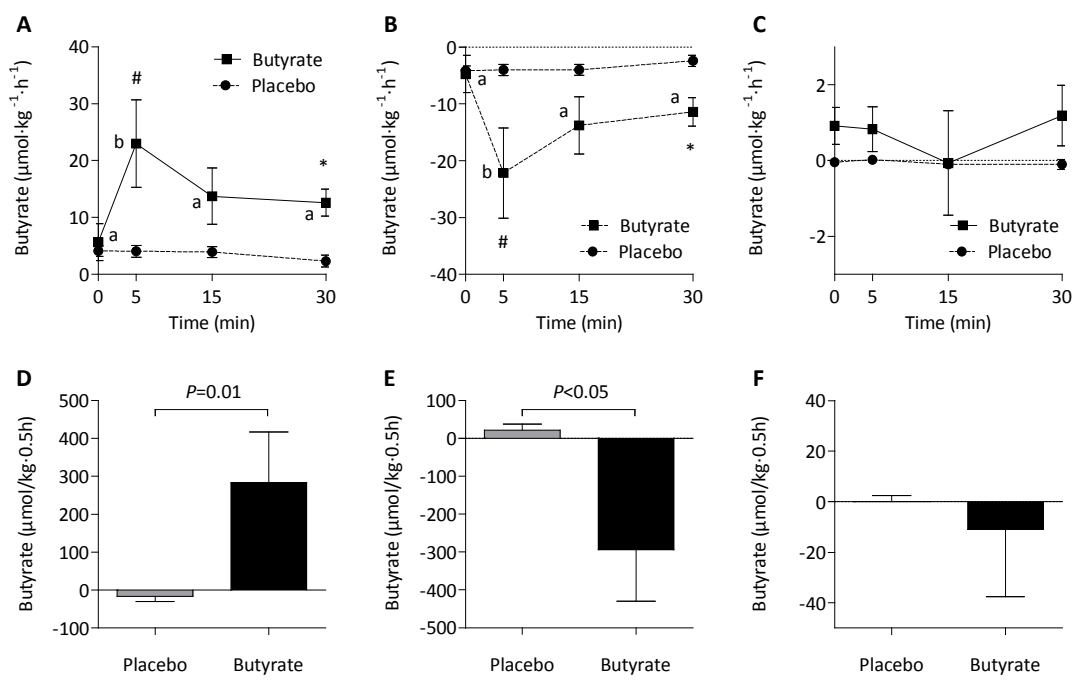

Butyrate exchange across gut (A and $\mathbf{D}$ ), liver (B and $\mathbf{E}$ ), and splanchnic area (C and $\mathbf{F}$ ) and corresponding incremental area under the curve (iAUC) following placebo and butyrate enema. A: Overall intervention effect after butyrate enema, $P=0.05, \mathrm{~B}$ : Overall intervention effect after butyrate enema, $P<0.01, C$ : Overall intervention effect after butyrate enema, $P=0.1$. *Different from placebo at respective time point, $P<0.05$. Labeled means $(a, b)$ within a group without a common letter differ, $P<0.05$. Values are means $\pm S E M, n=5$ (placebo) or $n=7$ (butyrate).

As gut release of butyrate did not exceed hepatic uptake in the butyrate-treated group, no net splanchnic butyrate release was observed, nor did this differ significantly from placebo (Figure 6.3C and 6.3F).

\section{Acetate and propionate exchange}

Rectal butyrate or placebo administration did not influence arterial, portal venous nor hepatic venous concentrations of acetate and propionate (data not shown). The gut released acetate and propionate in both groups, but this release did not differ significantly from baseline concentrations after administration of the butyrate or placebo enema. In line, no significant differences were observed between the two treatments for gut release of acetate and propionate (Supplemental Figure 6.1). Hepatic uptake of acetate and propionate, as well as release by the splanchnic area was insignificant. 


\section{Fractional absorption in blood}

In the butyrate-treated group, $60 \mathrm{~mL}$ of $100 \mathrm{mmol} / \mathrm{L}$ butyrate solution was administered, which equals $6 \mathrm{mmol}$ of butyrate. As mentioned above, portal venous butyrate concentrations increased after butyrate administration, and remained higher in the butyrate-treated group compared with placebo (Figure 6.2A). In total, measured as iAUC, $1.1 \pm 0.5 \mathrm{mmol}$ of butyrate was released from the gut into the portal vein within 30 min after administration of the butyrate enema. This was $18.3 \%$ of the total butyrate administered with the enema.

\section{DISCUSSION}

In this blinded randomized placebo-controlled human intervention study, we investigated 12 patients undergoing upper abdominal surgery and randomly assigned them to receive either a placebo or butyrate enema. The data showed an increased release of butyrate from the gut shortly after rectal administration of butyrate, combined with an increased butyrate uptake by the liver. As a result, there was no splanchnic release of butyrate in these subjects. Acetate and propionate fluxes did not change after butyrate administration, suggesting that there is no co-transport of acetate and propionate.

The experimental set-up during upper abdominal surgery in humans provides the unique opportunity to administer butyrate rectally with simultaneous blood sampling from the portal vein and a hepatic vein, as well as the radial artery. Together with our previous studies, these data give insight in human inter-organ SCFA trafficking. Baseline arterial, portal and hepatic venous concentrations found in the present study are comparable with data from previous studies, including our own data. ${ }^{20,21,27}$

High systemic concentrations of butyrate might be harmful.22,23,28 However, the exact plasma concentration at which butyrate becomes harmful is unknown. Studies administering precursors of SCFAs such as inulin or fructo-oligosaccharides reported increased intraluminal SCFA concentrations, but data about systemic changes have not been reported. ${ }^{29-31}$ In the current study, we used enemas that have previously been used in our institute. ${ }^{18}$ Prior to use of prebiotics or SCFAs in clinical settings, it had to be confirmed that this dose of butyrate can be administered safely to humans. Our data suggest that the liver is capable of metabolizing rectally administered butyrate rapidly, thereby preventing an increase in circulating butyrate concentrations. Specifically, rectally administered butyrate can be taken up by the colon and subsequently drains into the portal vein. Via the portal vein, butyrate reaches the liver, where it can be extracted and metabolized into acetyl-CoA by mitochondrial $\beta$-oxidation. The liver has a large capacity of metabolizing butyrate, as can be concluded from our previous study where we found that the cirrhotic liver is able to take up butyrate in a similar extent as a healthy liver. ${ }^{20}$ In the present study, as well as after prolonged administration, ${ }^{18}$ no adverse effects were observed. These findings 
support the assumption that it is safe to rectally administer clinically relevant butyrate concentrations, as used in this study.

During the study, we administered 6 mmol of butyrate, of which 18.3\% appeared in the portal vein within 30 min after administration. SCFAs are rapidly and almost completely absorbed by colonocytes, and only 5 - 10\% of the SCFAs are excreted by the feces. ${ }^{32,33}$ Absorption of SCFAs is concentration-dependent, and the absorption rates are the same throughout the entire colon. ${ }^{8}$ Two mechanisms have been described to be responsible for absorption of SCFA: passive diffusion of protonated SCFAs and active transport of SCFA anions. ${ }^{34}$ Passive diffusion of SCFAs plays a minor role, whereas active transport involving cellular uptake via different transporters, such as $\mathrm{HCO}_{3}$ - coupled receptors, $\mathrm{H}^{+}$-dependent monocarboxylate transporter isoform 1, MCT1 (SLC16A1), and Na+-coupled monocarboxylate transporter isoform 1, SMCT1 (SLC5A8) is probably more important. ${ }^{35}$ Although cellular transport is a rapid process, only $18.3 \%$ of the administered SCFAs were recovered in the portal vein in the present study. This may be explained by the fact that the colonic epithelium uses butyrate directly as an energy source and therefore the majority of butyrate may not appear in the portal vein. In fact, some studies indicate that up to $85-90 \%$ of butyrate is metabolized by the colonocytes. ${ }^{36}$ Furthermore, diffusion of butyrate is concentration-dependent. As long as the concentration difference between the colonic lumen and the blood is large enough, there is a driving force and the result is uptake of butyrate by the blood. Last, the veins that return from the rectal area constitute of veins draining into the portal vein as well as veins draining into the internal iliac vein. In the current study, only the amount of butyrate draining into the portal vein was measured. This can explain the relatively low fractional extraction of butyrate from the intestinal lumen into the portal vein, as well as the slight but significant increase in arterial butyrate concentrations after rectal butyrate administration, while there was no significant splanchnic release of butyrate.

This study has some limitations. First, reliable data about plasma flow are needed for flux calculations. As described above, in this study, plasma flow data of a previous study, performed in the same operative setting with comparable patients, were used. ${ }^{25}$ Variable data have been reported on portal plasma flows ranging from 423 - $1129 \mathrm{~mL} /$ min and hepatic artery flows ranging from 74 - $337 \mathrm{~mL} / \mathrm{min}$ when using Duplex flow measurements. ${ }^{37,38}$ Duplex flow measurements have considerable inter- and intra-observer variability, which introduces considerable variation. ${ }^{39,40}$ However, perioperative sonographic evaluation is time consuming and plasma flow is not easy to assess at any time point during surgery without interfering with the surgical procedures. In this context, it is important to point out that, by using a novel MRI-based flow measurement in humans, our group recently confirmed that mean flows are interchangeable and therefore mean plasma flow values can be used in flux calculations. ${ }^{38}$ Second, we only investigated 12 patients in this protocol, of which 7 received the butyrate enema and 5 the placebo enema. Due to the invasive protocol and complex study population, we anticipated that this would be enough 
to detect differences in butyrate concentrations and fluxes, despite large variations in physiological SCFA concentrations. On the other hand, we did see a similar flux pattern in all individual subjects; therefore we feel that this number of patients is sufficient to draw conclusions from this study.

With this study we showed that the human liver is capable to metabolize all butyrate after administration of a $6 \mathrm{mmol}$ butyrate enema. The fact that butyrate delivered in the distal part of the colon and rectum can bypass the liver via the rectal veins may explain the increase in arterial butyrate. However, SCFAs taken up at the proximal part of the colon will enter the portal vein and encounter the liver. Since prebiotics can be used to increase SCFA concentrations in the proximal part of the colon, this is physiologically important. Most fermentation of prebiotics into SCFAs takes place in the proximal part of the colon, since most substrate is available there. ${ }^{41}$ It can be anticipated that the part of the SCFAs that is not used by the proximal colonocytes, is excreted into the portal vein and can be metabolized by the liver, preventing harmful systemic concentrations.

In conclusion, this randomized placebo-controlled study in patients undergoing upper abdominal surgery showed the effects of rectal butyrate administration on human inter-organ trafficking. A rapid increase in gut release of butyrate was observed, which was paralleled by hepatic uptake of this SCFA. Consequently, no net splanchnic butyrate release was detected after administrating the butyrate enema. Arterial butyrate concentrations only slightly increased, and there was no significant effect on acetate and propionate organ fluxes. From these findings, we can conclude that the liver is capable of metabolizing butyrate delivered to the gut, preventing an increase in systemic butyrate concentrations. These findings are considered important when using prebiotic supplementation or local SCFA application on a therapeutic basis for colonic diseases. 


\section{REFERENCES}

1. Wong JM, de Souza R, Kendall CW, et al. Colonic health: fermentation and short chain fatty acids. Journal of Clinical Gastroenterology 2006;40:235-43.

2. Willemsen LE, Koetsier MA, van Deventer SJ, et al. Short chain fatty acids stimulate epithelial mucin 2 expression through differential effects on prostaglandin E(1) and E(2) production by intestinal myofibroblasts. Gut 2003;52:1442-7.

3. Hamer HM, Jonkers D, Venema K, et al. Review article: the role of butyrate on colonic function. Alimentary Pharmacology \& Therapeutics 2008;27:104-19.

4. Roediger WE. Role of anaerobic bacteria in the metabolic welfare of the colonic mucosa in man. Gut 1980;21:793-8.

5. Roediger WE. Utilization of nutrients by isolated epithelial cells of the rat colon. Gastroenterology 1982;83:424-9.

6. Lupton JR. Microbial degradation products influence colon cancer risk: the butyrate controversy. Journal of Nutrition 2004;134:479-82.

7. Forchielli ML, Walker WA. The role of gut-associated lymphoid tissues and mucosal defence. The British Journal of Nutrition 2005;93 Suppl 1:S41-8.

8. Roy CC, Kien CL, Bouthillier L, et al. Short-chain fatty acids: ready for prime time? Nutrition in Clinical Practice 2006;21:351-66.

9. Welters CF, Heineman E, Thunnissen FB, et al. Effect of dietary inulin supplementation on inflammation of pouch mucosa in patients with an ileal pouch-anal anastomosis. Diseases of the Colon and Rectum 2002;45:621-7.

10. Hille A, Herrmann MK, Kertesz T, et al. Sodium butyrate enemas in the treatment of acute radiation-induced proctitis in patients with prostate cancer and the impact on late proctitis. A prospective evaluation. Strahlentherapie und Onkologie 2008;184:686-92.

11. Kles KA, Chang EB. Short-chain fatty acids impact on intestinal adaptation, inflammation, carcinoma, and failure. Gastroenterology 2006;130:5100-5.

12. Leenen $\mathrm{CH}$, Dieleman LA. Inulin and oligofructose in chronic inflammatory bowel disease. Journal of Nutrition 2007;137:2572S-2575S.

13. Harig JM, Soergel KH, Komorowski RA, et al. Treatment of diversion colitis with short-chainfatty acid irrigation. New England Journal of Medicine 1989;320:23-8.

14. Guillemot F, Colombel JF, Neut C, et al. Treatment of diversion colitis by short-chain fatty acids. Prospective and double-blind study. Diseases of the Colon and Rectum 1991;34:861-4.

15. Roediger WE. The colonic epithelium in ulcerative colitis: an energy-deficiency disease? Lancet 1980;2:712-5.

16. Breuer RI, Buto SK, Christ ML, et al. Rectal irrigation with short-chain fatty acids for distal ulcerative colitis. Preliminary report. Digestive Diseases and Sciences 1991;36:185-7.

17. Scheppach W, Sommer H, Kirchner T, et al. Effect of butyrate enemas on the colonic mucosa in distal ulcerative colitis. Gastroenterology 1992;103:51-6.

18. Hamer HM, Jonkers DM, Vanhoutvin SA, et al. Effect of butyrate enemas on inflammation and antioxidant status in the colonic mucosa of patients with ulcerative colitis in remission. Clinical Nutrition 2010;29:738-44.

19. Cummings JH, Pomare EW, Branch WJ, et al. Short chain fatty acids in human large intestine, portal, hepatic and venous blood. Gut 1987;28:1221-7. 
20. Bloemen JG, Olde Damink SW, Venema K, et al. Short chain fatty acids exchange: Is the cirrhotic, dysfunctional liver still able to clear them? Clinical Nutrition 2009.

21. Bloemen JG, Venema K, van de Poll MC, et al. Short chain fatty acids exchange across the gut and liver in humans measured at surgery. Clinical Nutrition 2009;28:657-61.

22. Blau CA, Constantoulakis P, Shaw CM, et al. Fetal hemoglobin induction with butyric acid: efficacy and toxicity. Blood 1993;81:529-37.

23. Sher GD, Ginder GD, Little J, et al. Extended therapy with intravenous arginine butyrate in patients with beta-hemoglobinopathies. N Engl J Med 1995;332:1606-10.

24. van de Poll MC, Ligthart-Melis GC, Boelens PG, et al. Intestinal and hepatic metabolism of glutamine and citrulline in humans. J Physiol 2007;581:819-27.

25. van de Poll MC, Siroen MP, van Leeuwen PA, et al. Interorgan amino acid exchange in humans: consequences for arginine and citrulline metabolism. The American Journal of Clinical Nutrition 2007;85:167-72.

26. van Eijk HM, Bloemen JG, Dejong CH. Application of liquid chromatography-mass spectrometry to measure short chain fatty acids in blood. The Journal of Chromatography B 2009;877:719-24.

27. Cummings J, Pomare E, Branch W, et al. Short chain fatty acids in human large intestine, portal, hepatic and venous blood. Gut 1987;28:1221.

28. Grunert SC, Mullerleile S, De Silva L, et al. Propionic acidemia: clinical course and outcome in 55 pediatric and adolescent patients. Orphanet Journal of Rare Diseases 2013;8:6.

29. Duncan SH, Belenguer A, Holtrop G, et al. Reduced dietary intake of carbohydrates by obese subjects results in decreased concentrations of butyrate and butyrate-producing bacteria in feces. Applied and Environmental Microbiology 2007;73:1073-8.

30. Matsumoto N, Riley S, Fraser D, et al. Butyrate modulates TGF-beta1 generation and function: potential renal benefit for Acacia(sen) SUPERGUM (gum arabic)? Kidney International 2006;69:257-65.

31. Fernandes J, Morali G, Wolever TM, et al. Effect of acute lactulose administration on serum acetate levels in cirrhosis. Clinical \& Investigative Medicine 1994;17:218-25.

32. McNeil NI, Cummings JH, James WP. Short chain fatty acid absorption by the human large intestine. Gut 1978;19:819-22.

33. Roberfroid MB. Introducing inulin-type fructans. The British Journal of Nutrition 2005;93 Suppl 1:S13-25.

34. Cook SI, Sellin JH. Review article: short chain fatty acids in health and disease. Alimentary Pharmacology \& Therapeutics 1998;12:499-507.

35. den Besten G, van Eunen K, Groen AK, et al. The role of short-chain fatty acids in the interplay between diet, gut microbiota, and host energy metabolism. J Lipid Res 2013;54:2325-40.

36. Bach Knudsen KE, Serena A, Canibe N, et al. New insight into butyrate metabolism. Proceedings of the Nutrition Society 2003;62:81-86.

37. Yzet T, Bouzerar R, Allart JD, et al. Hepatic vascular flow measurements by phase contrast MRI and doppler echography: a comparative and reproducibility study. Journal of Magnetic Resonance Imaging 2010;31:579-88.

38. Vermeulen MA, Ligthart-Melis GC, Buijsman R, et al. Accurate perioperative flow measurement of the portal vein and hepatic and renal artery: A role for preoperative MRI? European Journal of Radiology 2011. 
39. de Vries PJ, van Hattum J, Hoekstra JB, et al. Duplex Doppler measurements of portal venous flow in normal subjects. Inter- and intra-observer variability. Journal of Hepatology 1991;13:358-63.

40. Carlisle KM, Halliwell M, Read AE, et al. Estimation of total hepatic blood flow by duplex ultrasound. Gut 1992;33:92-7.

41. Topping DL, Clifton PM. Short-chain fatty acids and human colonic function: roles of resistant starch and nonstarch polysaccharides. Physiol Rev 2001;81:1031-64. 


\section{SUPPLEMENTAL FIGURE}

Supplemental Figure 6.1. Acetate and propionate exchange across gut, liver, and splanchnic area

A

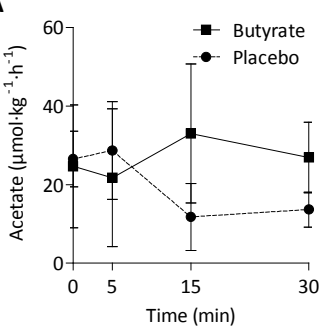

D

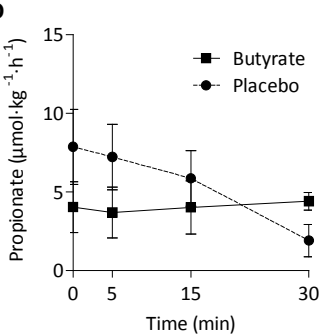

B

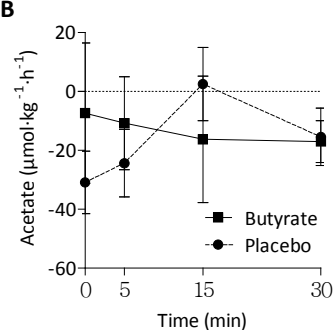

E

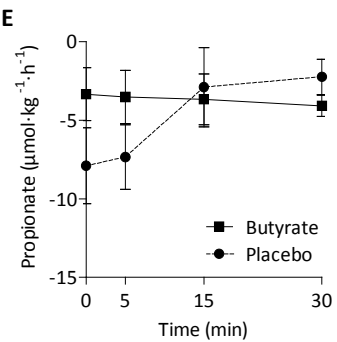

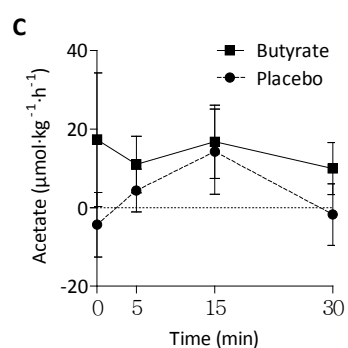

$\mathbf{F}$

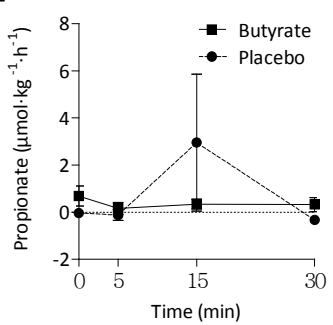

Acetate and propionate exchange across gut (A and $\mathbf{D})$, liver (B and $\mathbf{E}$ ) and splanchnic area ( $\mathbf{C}$ and $\mathbf{F}$ ) in human subjects receiving a butyrate or placebo enema during upper abdominal surgery. Values are means \pm SEM, $n=5$ (placebo) or $n=7$ (butyrate). There were no differences between the groups. 


$$
7
$$




\title{
CHAPTER ?
}

\section{Role of short-chain fatty acids in colonic inflammation, carcinogenesis, and mucosal protection and healing}

\author{
Christina M. van der Beek, Cornelis H. C. Dejong, Freddy J. Troost, \\ Ad A. M. Masclee, Kaatje Lenaerts
}

Nutrition Reviews. 2017;75(4):286-305 


\section{ABSTRACT}

Short-chain fatty acids (SCFAs), mainly acetate, propionate, and butyrate, produced by microbial fermentation of undigested food substances are believed to play a beneficial role in human gut health. Short-chain fatty acids influence colonic health through various mechanisms. In vitro and ex vivo studies show that SCFAs have anti-inflammatory and anti-carcinogenic effects, play an important role in maintaining metabolic homeostasis in colonocytes, and protect colonocytes from external harm. Animal studies have found substantial positive effects of SCFAs or dietary fiber on colonic disease, but convincing human evidence is lacking. Most human intervention trials have been conducted in the context of inflammatory bowel disease. Only a limited number of those trials are of high quality, showing little or no favorable effect of SCFA treatment over placebo. Opportunities for future research include exploring the use of combination therapies with anti-inflammatory drugs, prebiotics, or probiotics, the use of prodrugs in the setting of carcinogenesis, or the direct application of SCFAs to improve mucosal healing after colonic surgery. 


\section{INTRODUCTION}

The gut microbiota is rapidly generating interest worldwide as a result of the increasing awareness that its composition is closely associated with the development and progression of a variety of disorders. In addition, dysbiosis of the gut microbiota, i.e. a disturbance or imbalance in the microbial composition and diversity, has been reported to be associated with the pathogenesis of gastrointestinal disorders. ${ }^{1}$ Therefore, the question arises whether the gut microbiota plays a role in the etiology, onset, and maintenance of various colonic diseases, such as inflammatory bowel disease or colon cancer. Moreover, it is important to determine whether the microbiota plays a role in mucosal healing, a process essential for maintaining colonic health.

One of the major functions of the gut microbiota is the fermentation of undigested food components into short-chain fatty acids (SCFAs), primarily acetate, propionate, and butyrate. Dietary fibers are the most potent substrates for generating SCFAs. ${ }^{2}$ The rate, amount, and type of SCFAs produced are determined by several factors, including the substrate source, the colonic $\mathrm{pH}$, the abundance and composition of gut microbes, and the gut transit time. ${ }^{3}$ Depending on these factors, SCFAs can contribute up to $10 \%$ of the total human caloric requirement. ${ }^{4}$ Short-chain fatty acids are rapidly produced and are subsequently taken up by the colon, playing a pivotal role in colonic health. ${ }^{5-7}$

Of the three main SCFAs, butyrate is considered most important for maintenance of colonic health, primarily by acting as a direct source of energy for colonocytes. Butyrate is almost completely metabolized by colonocytes and is therefore found only in small amounts in peripheral blood. ${ }^{3}$ Acetate and propionate also have roles, although smaller, in maintaining colonic health. Most of the acetate, which is formed in higher quantities than the other SCFAs, is rapidly absorbed in the proximal colon and transported to the liver, where it can be used as a substrate for cholesterol synthesis. ${ }^{3}$ A recent study showed that less than $1 \%$ of the acetate administered colonically was incorporated into cholesterol and less than $15 \%$ into fatty acids. ${ }^{8}$ About $90 \%$ of the propionate produced is transported to the liver, where it functions as a substrate for gluconeogenesis, lipogenesis, and protein synthesis. ${ }^{3}$ Importantly, SCFAs act not only as single components but also influence each other's production and function. ${ }^{9}$ In addition, there is a high rate of conversion between the three SCFAs, especially from acetate into butyrate. 8,10

Currently, there is increasing interest in the therapeutic application of SCFAs in colonic diseases. Several in vitro, ex vivo, and animal studies show positive effects of SCFAs in reducing colonic inflammation, preventing carcinogenesis, and promoting anastomotic healing of the gut. It is unknown, however, whether these results can be translated into clinical practice. This review summarizes the effects of SCFAs reported in in vitro studies and animal studies and links the findings to human clinical trials conducted thus far. 


\section{PHYSIOLOGY}

\section{SCFAs in the colon and in the systemic circulation}

Short-chain fatty acids are produced predominantly in the cecum and in the proximal part of the colon, where most substrates are available for bacterial fermentation. These substrates become increasingly depleted along the colon toward the distal part, resulting in decreasing SCFA production. In individuals who died suddenly, total SCFA concentrations were found to be $131 \mathrm{mM}$ in the cecum and $80 \mathrm{mM}$ in the descending colon. ${ }^{11}$ However, in individuals who consume a high-fiber diet, concentrations in the proximal colon can reach up to $226 \mathrm{mM}$ acetate, $104 \mathrm{mM}$ propionate, and $78 \mathrm{mM}$ butyrate. ${ }^{12,13}$ The ratio of acetate, butyrate, and propionate throughout the colon is $3: 1: 1$, and it is similar in both the proximal and the distal colon, reflecting a similar uptake of all three SCFAs along the large bowel. ${ }^{11,14}$ The absorption of SCFAs is a quick, efficient, concentration-dependent process, and only $5-10 \%$ of the total amount of SCFAs present in the proximal large bowel are excreted by the feces. ${ }^{2}$ It is important to note that fecal concentrations of SFCAs do not reflect intracolonic concentrations or rates of SCFA production but are a net result of rates of SCFA production and intestinal absorption; hence, these concentrations should be interpreted with care. ${ }^{15}$

Short-chain fatty acids that have not been metabolized by colonocytes reach the liver via the portal vein. In addition, some of the SCFAs in the rectum may bypass the liver, as the venous drainage of the rectum occurs partly via the internal iliac vein directly into the systemic circulation. Since butyrate serves as an important energy source for colonocytes, only a small amount of butyrate reaches the hepatic system and is found in the systemic circulation under physiological conditions. ${ }^{8}$ A study in humans undergoing major abdominal surgery showed that the liver is capable of metabolizing large amounts of butyrate administered via an enema, thereby preventing splanchnic release of butyrate and a subsequent increase in systemic butyrate concentrations. ${ }^{16}$ Propionate and acetate released by the gut are processed by the liver. Acetate is the only SCFA that, under normal circumstances, can be found in considerable amounts in peripheral blood in humans. 8,9,17

\section{Transport and signaling of SCFAs in the gut}

Short-chain fatty acids are taken up in the colon via various transport systems. Apical uptake occurs through simple passive diffusion, ${ }^{18}$ but the largest part of SCFAs is taken up via several active transport mechanisms: exchange of SCFAs with $\mathrm{HCO}_{3}{ }^{-}, \mathrm{H}^{+}$-dependent cotransport via monocarboxylate transporter (MCT) isoform 1 (MCT1, or SLC16A1), and coupling of SCFAs with sodium via sodium-coupled monocarboxylate transporter (SMCT) isoform 1 (SMCT1, or SLC5A8). ${ }^{19-21}$ Normal functioning of these transporters is critical for optimal utilization of SCFAs by colonocytes. The fraction of total SCFAs that is not 
metabolized by the colonocytes is transported over the basolateral membrane to reach the systemic circulation via $\mathrm{HCO}_{3}{ }^{-}$exchange ${ }^{22}$ or cotransport via other $\mathrm{MCT}$ isoforms, namely MCT4 (SLC16A3) and MCT5 (SLC16A4). ${ }^{23}$

In addition to being taken up and metabolized by colonocytes, SCFAs bind to different colonic receptors to act as signaling molecules. ${ }^{7}$ In 2003, two G-coupled protein receptors (GPRs) for SCFAs were discovered, namely GPR41 and GPR43 (FFA3 and FFA2 receptors, respectively). ${ }^{24,25}$ Later, Tazoe et al.26,27 showed that these receptors are expressed in colonic mucosa. The relative potencies of the different SCFAs to interact with GPR43 are similar, but, for the GPR41 receptor, propionate is more potent than butyrate and is far more potent than acetate. ${ }^{24}$ Both receptors are expressed primarily by the enteroendocrine L-cells in the intestine, but they are also expressed by mast cells and leukocytes and thereby play an important role in the inflammatory response of the gut. After binding of SCFAs to GPR41 and GPR43, downstream signaling pathways such as mitogen-activated protein kinase signaling (MAPK) are activated, leading to, for example, the production of chemokines and cytokines and the regulation of cell proliferation. 25,28

More recently, another GPR for butyrate, GPR109a, was discovered. This receptor is expressed in the apical membrane of both colonocytes and enterocytes, as well as on colonic immune cells. ${ }^{29}$ GPR109a regulates inflammatory processes by suppressing the expression of nuclear factor $\mathrm{kB}(\mathrm{NF}-\mathrm{kB})^{29}$ and promoting the differentiation of colonic regulatory $T\left(T_{\text {reg }}\right)$ cells. ${ }^{30}$ 


\section{MECHANISMS OF ACTION OF SCFAS AT THE CELLULAR LEVEL}

The complex effects of SCFAs in colonic health and disease are ascribed to different mechanisms (Figure 7.1). Short-chain fatty acids have already been shown to provide energy to colonocytes and bind to GPRs to activate downstream signaling pathways. They also inhibit histone deacetylases (HDACs) in colonocytes and mucosal immune cells. Histone deacetylases are enzymes that prevent DNA transcription and thereby regulate gene expression. Studies in colon cancer cells have shown that butyrate is the most potent HDAC inhibitor. Propionate has a moderate effect on HDAC inhibition in the colon, but acetate has no effect. ${ }^{31}$ The following section describes several mechanisms through which SCFAs affect colonic health and disease.

\section{Metabolic homeostasis and protection of the colon}

In a healthy colon, it is important that colonocytes are protected from external harm so they can grow and proliferate in a normal manner, and preserve metabolic homeostasis. After colonic surgery, an optimal colonic environment is essential for re-epithelialization and mucosal healing. There are several ways that SCFAs protect colonocytes and maintain colonic homeostasis. Once absorbed, SCFAs, especially butyrate, become an important energy source for colonocytes, allowing them to grow and proliferate. ${ }^{32-34}$ In fact, oxidation of butyrate accounted for more than $70 \%$ of the oxygen consumption in colon biopsy specimens, indicating that colonocytes rely more on butyrate oxidation than on glucose as their primary energy source. ${ }^{35}$ In addition, in order to increase metabolic capacity of the colonocytes, SCFAs increase blood flow in the colonic mucosa by exerting a dilatory effect on arteriolar walls and increase oxygen uptake by colonocytes. ${ }^{36-38}$

Several studies have shown that SCFAs activate 5 ' adenosine monophosphate kinase (AMPK), a key player in the regulation of energy metabolism. Activation of AMPK in human colonocytes leads to tight-junction assembly and, thus, a stronger and healthier intestinal barrier. ${ }^{39-41}$ Indeed, several in vitro ${ }^{42}$ and ex vivo ${ }^{43}$ studies have indicated an improved colonic barrier function after SCFA supplementation. ${ }^{44}$ In addition, the administration of SCFAs results in increased cell membrane assembly and mucosal cell migration and enhanced proliferation and differentiation of healthy colonocytes. ${ }^{45-49} \mathrm{~A}$ healthy intestinal barrier not only enhances mucosal healing but also reduces the chance of colonization and infection by gut bacteria. ${ }^{50}$ Importantly, the intestinal barrier helps maintain colonic health, since a strong barrier decreases the possibility of tumor invasion, cell migration, formation of metastases, and inflammation. ${ }^{51-53}$ 
Figure 7.1. Complex effects of short-chain fatty acids via different mechanisms.

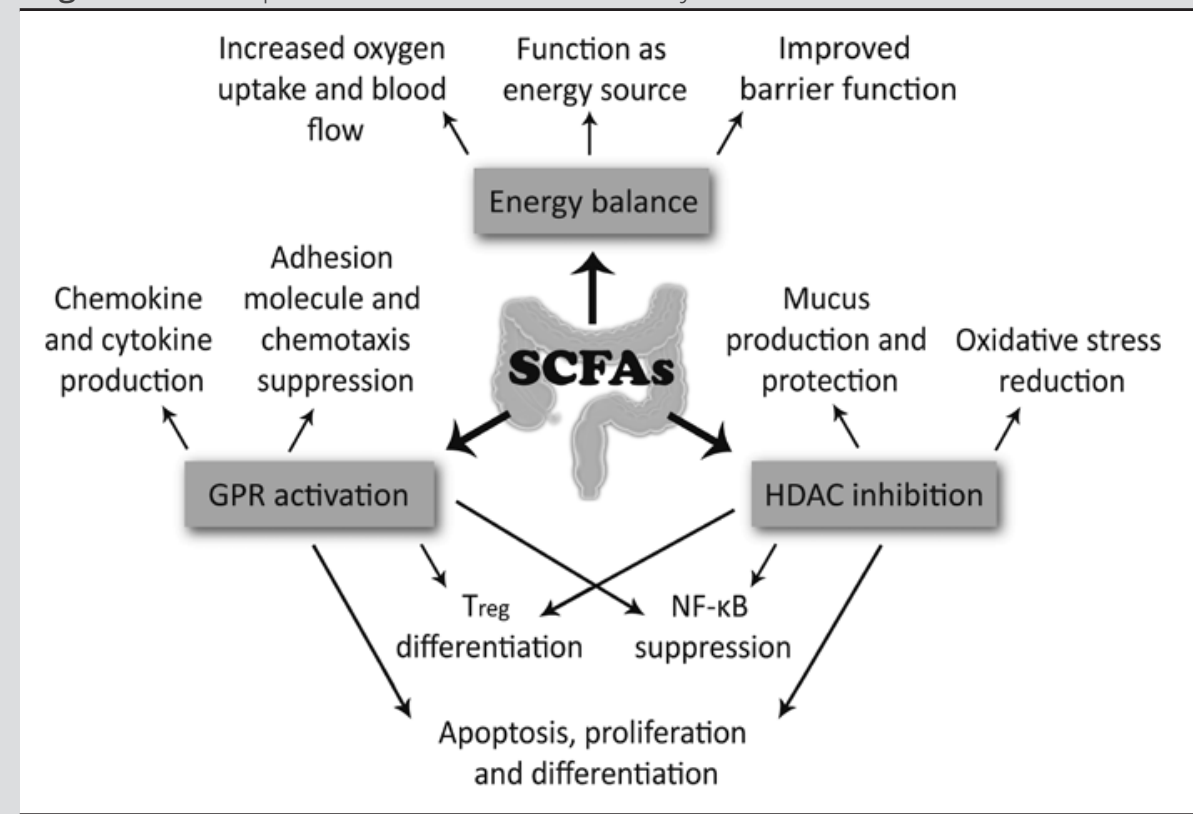

Abbreviations: GPR, G-coupled protein receptor; HDAC, histone deacetylase; NF-kB, nuclear factor $\mathrm{kB}$; $\mathrm{T}_{\text {reg' }}$, regulatory $\mathrm{T}$ cells.

Not only can SCFAs help rebuild the colonic epithelium, they can also protect it from external harm, such as caused by bacteria, reactive oxygen species, or immune-modulating prostaglandins. To protect the epithelial cells, the mucosa of the gastrointestinal tract is covered by a mucus layer that contains immune cells and protects the underlying epithelium against potentially harmful compounds. Mucus consists of different mucins, of which epithelial mucin 2 (MUC2), encoded by the MUC2 gene, is the most prominent in both the healthy and the inflamed gut. ${ }^{54-56}$ In vitro studies showed that HDAC inhibition via SCFA supplementation enhanced MUC2 expression in intestinal epithelial cells, ${ }^{54,57-59}$ and exposure of human colonic tissue to butyrate increased mucus synthesis. ${ }^{60}$ Administration of SCFAs increases not only MUC2 expression but also the expression of other mucins, such as MUC1, MUC3, and MUC4.61,62

Besides the physical barrier of mucus to protect the epithelium, SCFAs can also protect the epithelium by modulating oxidative stress and immune modulators. Evidence that SCFAs can modulate oxidative stress, possibly via HDAC inhibition, is accumulating. ${ }^{63}$ Oxidative stress is defined as an imbalance between reactive oxygen species and antioxidant compounds in the body. Reactive oxygen species can be scavenged by antioxidants, such as vitamins, glutathione, uric acid, and ubiquinol. ${ }^{64}$ Importantly, a large prospective 
human study showed that higher serum concentrations of reactive oxygen species are associated with an increased risk of developing colorectal cancer. ${ }^{65}$ Moreover, oxidative stress induces colonic inflammation. ${ }^{66-68}$ In vitro studies showed that butyrate and a physiological mixture of acetate, propionate, and butyrate protects colonocytes from DNA damage induced by reactive oxygen species. ${ }^{69,70}$ In addition, SCFAs protect the mucus layer from injury by reducing levels of immune modulators, such as prostaglandins formed by cyclooxygenase-2 (COX-2). Prostaglandin E2 promotes inflammation and tumor growth in colon cancers, ${ }^{71}$ and levels of COX-2 mRNA are higher in colon cancer tissues. ${ }^{72}$ In vitro studies show that butyrate is able to suppress COX-2 expression in cancerous tissues, thereby preventing the damaging effects of prostaglandins on the mucus layer..$^{73-75}$

Another important factor in maintenance of the epithelial barrier is hypoxia-inducible factor, a transcription factor that coordinates barrier protection. ${ }^{43}$ Loss of epithelial hypoxia-inducible factor has been shown to increase susceptibility to colitis.44,76 Interestingly, the addition of butyrate and other SCFAs to cultured epithelial cell lines enhanced local oxygen consumption, thereby stabilizing hypoxia-inducible factor and improving epithelial barrier function. ${ }^{77}$

\section{Colonic inflammation}

Short-chain fatty acids have been shown to have direct immune-modulatory effects via HDAC inhibition and GPR activation, as reviewed recently. ${ }^{44}$

First, SCFAs inhibit HDACs in colonocytes and mucosal immune cells, thereby regulating gene expression in these cells. ${ }^{78,79}$ Inhibition of HDACs leads to suppression of the nuclear transcription factor NF-kB in several cell types of the mucosal immune system, thereby influencing transcription of inflammatory-associated genes, including those that code for cytokines. ${ }^{80,81}$ In vivo studies have shown that SCFAs directly modulated Toll-like receptor 4 signaling, suppressed the production of pro-inflammatory cytokines tumor necrosis factor-a, interleukin (IL)-6, and IL-12, increased the production of the anti-inflammatory cytokine IL-10, and reduced the infiltration of colonic mucosa by leukocytes, thereby directly suppressing the immune response. ${ }^{82,83}$ Recently, SCFAs have also been shown to induce differentiation of mucosal $T_{\text {reg }}$ cells via HDAC inhibition. ${ }^{84,85} \mathrm{~T}_{\text {reg }}$ cells expressing transcription factor Foxp3 play an important role in limiting intestinal inflammatory responses via production of IL-10, and in vitro stimulation by SCFAs increased Foxp3 expression by $\mathrm{T}_{\text {reg }}$ cells. ${ }^{85}$ In addition, recent investigation has shown that SCFAs affect immune antibody response through HDAC inhibition. ${ }^{86}$ Indeed, in vitro application of SCFAs enhanced the generation of immunoglobulin-expressing B cells. ${ }^{86}$ The same study also showed that SCFAs increased acetyl-coenzyme A, and oxidative phosphorylation, glycolysis, and fatty acid synthesis to produce energy needed for antibody production. In practice, increased NF-KB activation was found in colonic biopsies from patients with Crohn's disease. This activation was inhibited by applying butyrate to the specimen and was accompanied by a 
reduction in the release of several pro-inflammatory cytokines. ${ }^{66,87}$

Second, SCFAs bind to different GPRs, i.e., GPR41, GPR43, and GPR109a, on the apical surface of colonocytes and immune cells, thereby activating several signaling pathways. In particular, GPR43 activation by acetate and propionate, and GPR109a activation by butyrate seem to be important factors in suppressing the inflammatory response. GPR43 activation and, in a less potent manner, GPR41 activation both reduce colonic inflammation. This is achieved by suppressing the expression of adhesion molecules in inflammatory cells and endothelial cells, ${ }^{88,89}$ by preventing chemotaxis of monocytes to the inflammatory site, ${ }^{90}$ by promoting chemokine and cytokine production, ${ }^{28}$ and by regulating $T_{\text {reg }}$ function. ${ }^{91}$ Butyrate-induced activation of GPR109a also affects the inflammatory process by suppressing NF-KB expression ${ }^{29}$ and promoting the differentiation of $\mathrm{T}_{\text {reg }}$ cells. $^{30}$

\section{Colon carcinogenesis}

Short-chain fatty acids can exert direct anti-carcinogenic effects through both HDAC inhibition and GPR activation. Inhibition of HDACs by SCFAs is associated with cell cycle arrest, causing anti-proliferative and pro-apoptotic effects as well as an increase in cell differentiation in colon cancer tissue. ${ }^{92} \mathrm{~A}$ microarray analysis of human colonic epithelial cells showed that most of the genes responsive to butyrate were indeed involved in the processes of apoptosis, proliferation, and differentiation..$^{93}$ Butyrate, propionate, and acetate increased apoptosis in several adenoma and carcinoma cells by stimulating the expression of the cell cycle regulatory genes p53 and p21, by decreasing the expression of the anti-apoptotic $\mathrm{BCl}-2$ protein, and by increasing the expression of the pro-apoptotic Bax protein. 33,34,94,95 Butyrate is the most potent inhibitor of cell proliferation, consistent with it being the most potent HDAC inhibitor, although acetate and propionate seem to augment the effects of butyrate in adenoma cells. ${ }^{96,97}$ Butyrate, propionate, and acetate promoted cell differentiation and inhibited cell migration in colon cancer cells in vitro, thereby reducing the invasiveness of carcinoma cells. ${ }^{97-101}$ In addition, GPR43 and GPR109a both act as tumor suppressors by inhibiting proliferation and increasing apoptosis in colon cancer cell lines, independent of HDAC inhibition. ${ }^{29,102}$ Moreover, butyrate and other HDAC inhibitors inhibit cell growth in colon cancer cell lines, independent of HDAC inhibition, via attenuation of Wnt signaling, a pathway that is active in most colorectal carcinomas and that induces cell proliferation and tumorigenesis in these carcinomas. ${ }^{103}$ Some colorectal cancer cells develop so-called butyrate resistance to the apoptotic effects of butyrate, which is related to a different degree of Wnt attenuation. ${ }^{104}$

The apparent contradiction in the effects of SCFAs, which provide energy for normal cells to grow and proliferate and yet also inhibit the proliferation of cancer cells, is called the butyrate paradox. ${ }^{105}$ It is explained by the Warburg effect: cancerous colonocytes rely on glycolysis for oxidation, even in the presence of SCFAs. Therefore, the accumulating SCFAs inhibit proliferation of cancerous colonocytes, while SCFA oxidation in unaffected 
cells stimulates proliferation, possibly by affecting different target genes. ${ }^{106,107}$ Another hypothesis proposes that the epithelial architecture, organized in crypts, protects stem cells from growth inhibition, since stem cells are located in the deep base of the crypt, where fewer SCFAs can reach them. ${ }^{108}$ In a carcinogenic state, in which the epithelial lining might be disrupted, SCFAs can reach the stem cells and suppress stem cell proliferation.

\section{EVIDENCE FROM ANIMAL STUDIES}

\section{Inflammation}

The important role of SCFAs in inflammation was demonstrated using SCFA receptor knockout mice and colitis models in animals. Several authors showed beneficial effects of supplementing SCFAs, mostly butyrate, in experimentally induced colitis. ${ }^{109-113}$ GPR43 activation via acetate treatment reduced colitis in mice. ${ }^{87,114,115}$ In addition, GPR43-deficient mice had a greater inflammatory response to the carcinogen dextran sodium sulfate than their wild-type littermates. The addition of acetate to the drinking water of the GPR43-deficient mice resulted in a substantial decrease in inflammation, showing that acetate could reduce inflammation also by pathways other than GPR43 activation. ${ }^{89}$ In an experimental model of diversion colitis in rats, a butyrate enema reduced inflammation, restored collagen, and restored the rate of apoptosis in the epithelium. ${ }^{116,117}$ The butyrate receptor GPR109a protected against the development of colitis and subsequent colon carcinoma in GPR109a knockout mice. ${ }^{30}$ Moreover, oral butyrate intake ameliorated colitis in mice by increasing $T_{\text {reg }}$ cell differentiation, ${ }^{84,118}$ and a butyrate enema lowered NF-kB expression in mice with colitis. ${ }^{112,113}$ These findings support the conclusion that the in vitro mechanisms of SCFAs are also applicable to animal studies.

Supplementation with dietary fiber, such as guar gum, potato fiber, enzyme-treated rice fiber, and others, reduced inflammation and resulted in improved barrier integrity and mucosal healing in chemically induced (dextran sodium sulfate or 2,4,6,-trinitrobenzene sulfonic acid) and genetic (IL-10-/-) animal models of colitis. ${ }^{119-124}$ In these studies, the beneficial effects of fiber on the intestine were associated with overt increases in luminal and/or fecal SCFA concentrations. In addition, the reduction of SCFA concentrations observed in colitis (dextran sodium sulfate model and IL-10-/- model) could be restored by treatment with dietary fiber. ${ }^{119,123}$ Moreover, feeding dietary fiber (pectin and inulin mixture) to mice resulted in improved host antibody responses and reduced susceptibility to pathogens, which appeared to be, at least in part, mediated by increased concentrations of SCFAs. ${ }^{86}$ In a validated rat model of inflammatory bowel disease, however, beneficial effects of the fibers inulin and fructo-oligosaccharides on intestinal inflammation were associated with concomitant changes in microbiota profiles, although cecal and fecal concentrations of SCFAs remained unchanged. ${ }^{125}$ Hence, dietary fiber may also improve intestinal homeo- 
stasis by generating and/or inhibiting other metabolites or by affecting the gut microbiota.

\section{Carcinogenesis}

The direct application of SCFAs to the colon might prevent the development of colon cancer. Some studies have confirmed the anti-carcinogenic effects of butyrate in animals, ${ }^{126-128}$ but other studies found no effect of butyrate on colon carcinogenesis. ${ }^{129-131}$ In these latter studies, however, only a low concentration of butyrate was used, which might explain the results observed, possibly indicating that a higher concentration is needed to be effective.

Since the direct application of SCFAs in the colon to protect against colon cancer is not physiological, some authors have investigated the use of prodrugs or dietary fibers combined with SCFAs to protect against colon cancer in animals. Prodrugs are inactive compounds that are metabolized by the body into active SCFAs. The advantage of the use of prodrugs is that they, in contrast to SCFAs, provide a stable and high concentration after rapid absorption at the site of administration, while SCFAs themselves are rapidly metabolized by the colonocytes. An example of a prodrug is tributyrin, an ester composed of glycerol and butyrate. Tributyrin is metabolized by intracellular lipases, releasing therapeutically effective butyrate over time directly into the cell. ${ }^{132}$ Recent studies show that tributyrin protects carcinogen-treated rats against the development of colon cancer. ${ }^{133}$ In addition, a study with carcinogen-treated mice showed a lower tumor incidence of $43 \%$ in tributyrin-fed mice compared with $60 \%$ in control mice. ${ }^{134}$ Clarke et al. ${ }^{135}$ showed that carcinogen-treated rats fed a butyrylated starch diet had more apoptosis in the distal colon when compared with rats fed a control diet.

It has been hypothesized that the dietary fiber-mediated suppression of colon carcinogenesis is attributable to butyrate-producing gut bacteria. In a mechanistic study using an inflammation-associated model of colon carcinogenesis in mice, absence of the SCFA receptor GPR43 (Ffar2-/-) resulted in decreased animal survival. Dietary fiber was able to suppress weight loss, diarrhea, and the development of colonic polyps, but only in wildtype mice and not in Ffar2-/- mice. These data indicate that the GPR43 receptor is crucial for the suppression of inflammation-associated colon carcinogenesis by dietary fiber. ${ }^{136}$ Interestingly, another mouse study revealed a role for the butyrate receptor GPR109a in this suppression. ${ }^{137}$ In rats exposed to a genotoxic challenge with azoxymethane, intake of butyrylated high-amylose maize starch resulted in higher total SCFAs, as well as acetate and butyrate concentrations, in the colon, and higher concentrations of total SCFAs, acetate, and butyrate in hepatic portal venous plasma, all in a dose-dependent manner. This intervention increased mucus thickness, decreased DNA single-strand breaks, and increased the rate of apoptosis in a dose-related manner in cells in the distal colon. ${ }^{138}$ The effects of dietary fiber are thought to be dependent on both the source of the fiber and the composition of the gut microbiota. To investigate this, Donohoe et al. ${ }^{139}$ used gnoto- 
biotic mouse models colonized with wild-type or mutant strains of a butyrate-producing bacterium to demonstrate that dietary fiber has a potent tumor-suppressive effect, but in a microbiota- and butyrate-dependent manner.

In conclusion, since the use of SCFA enemas in human interventions might not be feasible, the use of prodrugs or dietary fiber might allow the potent effects of SCFAs to be exploited in the prevention of colon cancer in humans. As noted below, whether dietary fiber protects against colorectal cancer is still controversial. It may be possible, however, to discriminate between individuals who respond to the anticancer effect of fiber and those who do not, on the basis of careful analysis of their microbiome. ${ }^{139}$

\section{Mucosal healing and colonic protection}

Van der Sluis et al. ${ }^{140}$ showed that MUC2-deficient mice spontaneously develop colitis and colon cancer, suggesting that the mucus layer has a critical role in colonic homeostasis and protection from external harm. Administering butyrate enemas to mice for 7 days increased MUC2 gene expression, but a decrease in mucus thickness was described. ${ }^{61}$ Ferreira et al. ${ }^{111}$ showed an improved intestinal barrier after adding either a mixture of SCFAs or butyrate alone to the drinking water of mice in a colitis model. Rolandelli et al. ${ }^{141}$ were the first to investigate the effects of SCFAs in mucosal healing after surgery. In their study, an anastomosis of the descending colon was made, and continuous colonic infusion with an SCFA mixture of acetate, propionate, and butyrate was administered. Rats without any colonic infusion and rats with colonic electrolyte infusion were used as controls. In the SCFA-treated group, the occurrence of spontaneous anastomotic dehiscence was considerably less, as was the occurrence of anastomotic suture bursting. Bursting pressure and bowel wall tension were higher in the SCFA-treated group than in either of the other groups, indicating that SCFAs in the anastomosed colon led to a stronger anastomosis and better mucosal healing. ${ }^{141}$ Other studies have confirmed these positive findings on colonic mucosal healing in rats. ${ }^{142,143}$ In a surgical model of acute colonic obstruction in rats, bursting pressure and bowel wall tension were higher in the SCFA-treated group, and bursting of the colon was more likely to occur in a non-operated part of the bowel, indicating that the operated site was actually stronger than the rest of the non-operated part of the colon. ${ }^{144}$ Another study of bowel obstruction in rats showed that intraoperative lavage with a SCFA mixture reduced the size of necrotic ulcers at the suture line and resulted in more rapid healing of the mucosal gap at the anastomosis. ${ }^{50}$ Again, other animal studies found positive effects of SCFAs on mucosal healing and prevention of adhesions. ${ }^{45,145-150}$ 


\section{HUMAN EPIDEMIOLOGICAL STUDIES}

Gut microbial dysbiosis might play a role in the onset or treatment of gastrointestinal disease. The consumption of specific carbohydrates influences the composition and function of the human microbiome. ${ }^{151}$ Because some bacterial species, such as Bifidobacterium, are more potent in generating SCFAs from complex carbohydrates than others, it can be postulated that a change in the microbial composition of the gut, as occurs, for example, by dietary modification or the use of antibiotics, can change both the use and the production of SCFAs. ${ }^{152}$ Therefore, microbial dysbiosis, which occurs in gastrointestinal disease, could lead to a change in the production and/or utilization of SCFAs.

The observation that patients with inflammatory bowel disease have a different fecal SCFA profile than healthy controls ${ }^{153}$ and the finding of a lower abundance of butyrate-producing bacteria in ulcerative colitis and Crohn's disease patients ${ }^{154-156}$ clearly demonstrate the relationship between microbial dysbiosis, SCFAs, and inflammatory bowel disease in humans. In addition, the number of butyrate-producing bacteria correlated negatively with disease activity in Crohn's disease patients. ${ }^{157}$ Fecal SCFA concentrations were also markedly lower in patients with pouchitis than in patients without pouchitis. ${ }^{158-162}$ In a small group of 11 pouch patients, low fecal butyrate appeared to be indicative of the development of pouchitis within the first year after pouch construction. ${ }^{158}$ In 1980, Roediger ${ }^{35}$ provided the first evidence that SCFAs might influence gut inflammation in humans by showing that colonocytes from patients with ulcerative colitis had a considerably lower capacity for butyrate oxidation when compared with colonocytes from healthy individuals. Importantly, bacterial butyrate and propionate metabolism was decreased in ileal Crohn's disease. ${ }^{163}$ Butyrate oxidation was found to be reduced in active, mild to moderate inflammatory disease in ex vivo studies; ${ }^{53,164}$ however, there seems to be no defect in butyrate oxidation in quiescent disease. ${ }^{164,165}$ In line with this, a recent study found that the inflammatory process itself induces impaired butyrate oxidation but that the inflammation is not a result of lower colonic butyrate concentrations. ${ }^{166}$ The observation that colonic concentrations of SCFAs, especially acetate, are lower when ulcerative colitis is active than when in remission supports the hypothesis that the inflammation itself might cause the impaired uptake and utilization of SCFAs, instead of SCFAs being the cause of the impairment. ${ }^{167,168}$ These findings imply that, in humans, the immunomodulatory effects of SCFAs on inflamed colonocytes are more important than the recovery of the metabolic deficiency in the inflamed colonocytes.

A role for SCFAs in the development of colon cancer was recently suggested by the downregulation of SCFA receptors MCT1, SMCT1, GPR43, and GPR109a in human colon cancer tissue. ${ }^{29,102,169,170}$ Brown et al. ${ }^{171}$ found significant perturbations in SCFA metabolism in colonic tissue from 17 colorectal cancer patients. In 1971, Burkitt ${ }^{172}$ stated that the lower 
intake of dietary fiber in developed countries compared with the intake in developing countries causes a difference in colon cancer prevalence. Several studies showed a lower incidence of colon cancer along with higher fecal concentrations of SCFAs in native Africans compared with African Americans and Caucasian Americans. ${ }^{173-176}$ In addition, the capacity of the colonic bacteria to ferment carbohydrates and to produce butyrate was found in patients with colon cancer or polyps. ${ }^{177-179}$ This all seems to support the hypothesis that a higher intake of complex carbohydrates leads to higher amounts of colonic SCFAs, thereby decreasing the risk of developing colon cancer. Patients with familial adenomatous polyposis are prone to develop colon cancer, since they have a chromosomal defect that initiates adenomatous changes in the gut. Compared with healthy individuals, patients with familial adenomatous polyposis produce fewer fecal SCFAs. ${ }^{180}$ When comparing familial adenomatous polyposis patients with and without colonic polyps, Bradburn et al. ${ }^{180}$ found that patients with polyps produced stool with larger ratios of acetate to butyrate and acetate to propionate. A higher proportion of acetate and a lower proportion of butyrate were also found in the feces of subjects with colon cancer or polyps compared with subjects without colonic disease. ${ }^{177,178}$ These studies, however, are all associative, and the role of SCFAs in colonic health and disease is best investigated in human intervention trials.

\section{CLINICAL HUMAN INTERVENTION TRIALS: INFLAMMATORY DISEASES}

Most clinical studies have been conducted in ulcerative colitis because it affects predominantly the distal part of the colon, which is easy to reach using enemas, and manifests as superficial mucosal inflammation. Ulcerative colitis is different than Crohn's disease, which may affect the entire gastrointestinal tract and causes deep ulcers that extend into all layers of the bowel wall, making treatment difficult. Some studies have investigated SCFA treatment in diversion colitis, radiation proctitis, and pouchitis. These diseases vary in pathophysiology, and thus different underlying mechanisms may be involved in treatment outcome.

\section{Ulcerative colitis}

Ulcerative colitis is a chronic inflammatory bowel disease that often manifests with symptoms such as diarrhea and rectal blood loss. It is characterized by episodes of active inflammation and periods of more quiescent disease during which patients can be relatively symptom free. The advantage of SCFA enemas is that they cause no side effects, in contrast to treatment with corticosteroids or mesalazine. Several studies have attempted to investigate the role of SCFA or butyrate enemas in the treatment of patients with 
ulcerative colitis. The results of early uncontrolled studies seemed promising, ${ }^{181-184}$ with symptom improvement reported after rectal administration of either butyrate or a SCFA combination therapy. For example, Breuer et al. ${ }^{181}$ showed in 1991 that an enema composed of the SCFAs acetate and propionate resulted in symptom improvement in patients with refractory ulcerative colitis. Moreover, a mixture of the SCFAs acetate, propionate, and butyrate improved symptoms in 9 of 10 patients. In addition, in a single-blinded crossover study, 10 patients with distal ulcerative colitis refractory or intolerant to standard therapy for 8 weeks received 2 weeks of butyrate enemas followed by 2 weeks of placebo with a 2-week washout in between. The butyrate enema had a positive effect on stool frequency, rectal blood loss, and endoscopic and histologic inflammation. ${ }^{185}$ One study evaluated the effectiveness of SCFA enemas vs mesalazine or corticosteroid enemas in 45 patients with nonspecific proctosigmoiditis, which is not always distinguishable from ulcerative colitis. ${ }^{186}$ The authors showed that SCFAs are equally effective at treating nonspecific proctosigmoiditis when compared with the other two treatments, but the study lacked a placebo group.

The outcomes of subsequent randomized, double-blind, placebo-controlled studies, however, were less impressive (Table 7.1).67,184,185,187-192

Most studies report no significant difference between SCFA application and placebo. ${ }^{67,188,189}$ The most convincing studies are those performed by Steinhart et $a l^{1{ }^{187}}$ and Breuer et al. ${ }^{190}$, who investigated 38 and 91 patients with ulcerative colitis, respectively. Both showed no significant effect of SCFA enemas on endoscopic or histologic scores or on clinical disease activity indexes. Only 2 studies from Vernia et al. ${ }^{192,193}$ showed improvement after rectal administration of SCFAs. The first study, conducted in 40 patients with mild to moderate ulcerative colitis, found an improvement in clinical signs, such as intestinal bleeding, urgency, and a patient self-evaluation score. No significant difference between butyrate and placebo was found for histologic or endoscopic scores. ${ }^{193}$ The second study investigated whether a combination of mesalazine and butyrate was more effective than mesalazine alone in 51 patients with active, mild to moderate ulcerative colitis refractory to standard therapy for 8 weeks. ${ }^{192}$ This study also found significant beneficial effects on clinical signs of active disease, such as on stool frequency, urgency, and the patient self-evaluation score. However, no significant effect on histologic or endoscopic scores was found.

The discrepancy between the studies might be due to a pronounced placebo effect, reported by almost all of the placebo-controlled studies. Most studies also describe the presence of specific responders and non-responders to treatment, which led Lührs et al. ${ }^{191}$ to use only a subset of patients who positively reacted to butyrate enemas in another study. ${ }^{188}$ It is possible that butyrate enemas improve the efficacy of medication, such as mesalazine, even when ingested orally using coated tablets. ${ }^{192-194}$ 
Table 7.1. Clinical placebo-controlled studies evaluating the effect of rectal treatment with short-chain fatty acids in ulcerative colitis

\begin{tabular}{|c|c|c|c|}
\hline Reference & No. of patients & Type of intervention and placebo & $\begin{array}{l}\text { Frequency and } \\
\text { duration of } \\
\text { treatment }\end{array}$ \\
\hline Vernia et al. ${ }^{184}$ & $\begin{array}{l}40 \text { patients with mild } \\
\text { to moderate UC } \\
\text { ( } 20 \text { treatment; } 20 \\
\text { placebo) }\end{array}$ & $\begin{array}{l}\text { Treatment: } 100 \mathrm{~mL} \text { of SCFA (acetate } \\
\text { at } 80 \mathrm{mmol} / \mathrm{L} \text {, propionate at } \\
30 \mathrm{mmol} / \mathrm{L} \text {, butyrate at } 40 \mathrm{mmol} / \mathrm{L} \text { ) } \\
\text { Placebo: } 100 \mathrm{~mL} \text { of isotonic } \mathrm{NaCl} \\
\text { Constant oral medication } \\
\text { (mesalazine, sulfasalazine) }\end{array}$ & Twice daily for 6 wk \\
\hline Scheppach et al. ${ }^{185}$ & $\begin{array}{l}10 \text { patients with } \\
\text { active UC refractory } \\
\text { to standard therapy } \\
\text { for } 8 \text { wk } \\
\text { Randomized } \\
\text { crossover design }\end{array}$ & $\begin{array}{l}\text { Treatment: } 100 \mathrm{~mL} \text { of butyrate at } \\
100 \mathrm{mmol} / \mathrm{L} \\
\text { Placebo: } 100 \mathrm{~mL} \text { of isotonic } \mathrm{NaCl} \\
\text { Constant oral medication } \\
\text { (mesalazine, sulfasalazine, } \\
\text { prednisone) }\end{array}$ & $\begin{array}{l}\text { Twice daily for } 2 \text { wk } \\
\text { 2-wk washout } \\
\text { period before } \\
\text { alternate enema } \\
\text { treatment }\end{array}$ \\
\hline Steinhart et al. ${ }^{187}$ & $\begin{array}{l}38 \text { patients with } \\
\text { active UC } \\
\text { (19 treatment; } 19 \\
\text { placebo) }\end{array}$ & $\begin{array}{l}\text { Treatment: } 60 \mathrm{~mL} \text { of butyrate at } \\
80 \mathrm{mmol} / \mathrm{L} \\
\text { Placebo: } 60 \mathrm{~mL} \text { of } \mathrm{NaCl} \text { enema + } \\
\text { butyrate at } 0.8 \mathrm{mmol} / \mathrm{L} \\
\text { Constant oral medication } \\
\text { (mesalazine, sulfasalazine, } \\
\text { prednisone) }\end{array}$ & $\begin{array}{l}\text { One enema nightly } \\
\text { for } 6 \text { wk }\end{array}$ \\
\hline Scheppach ${ }^{188}$ & $\begin{array}{l}47 \text { patients with } \\
\text { active UC (16 SCFA; } \\
15 \text { butyrate; } 16 \\
\text { placebo) }\end{array}$ & $\begin{array}{l}\text { Treatment 1: } 60 \mathrm{~mL} \text { of SCFA (acetate } \\
\text { at } 60 \mathrm{mmol} / \mathrm{L} \text {, propionate at } \\
30 \mathrm{mmol} / \mathrm{L} \text {, butyrate at } 40 \mathrm{mmol} / \mathrm{L} \text { ) } \\
\text { Treatment 2: } 60 \mathrm{~mL} \text { of butyrate at } \\
100 \mathrm{mmol} / \mathrm{L} \\
\text { Placebo: } 60 \mathrm{~mL} \text { of isotonic } \mathrm{NaCl} \\
\text { Constant oral medication } \\
\text { (mesalazine, sulfasalazine, } \\
\text { prednisone) }\end{array}$ & Twice daily for 8 wk \\
\hline
\end{tabular}

Abbreviations: NaCl, sodium chloride; NF-KB, nuclear factor-kappa B; NS, not significant; SCFAs, short-chain fatty acids; UC, ulcerative colitis; UCDAI, ulcerative colitis daily activity score. 
Disease remission or improvement in clinical symptoms (stool frequency, rectal bleeding, nocturnal bowel movements, urgency, and patient self-evaluation), endoscopic score (0-3), and histologic score (0-3)

Disease remission or marked improvement in clinical symptoms (stool frequency, rectal bleeding), endoscopic score (0-10), and histologic score (0-3)

\section{SCFAs:}

Reduced intestinal bleeding $\quad<0.05$

Less urgency $\quad<0.02$

Better patient self-evaluation score $\quad<0.05$

Endoscopic scores NS

Histologic scores NS

\section{Butyrate:}

Decrease in number of defecations $\quad<0.01$

Decrease in rectal bleeding $\quad<0.05$

Endoscopic scores $\quad<0.01$

Histologic scores $\quad<0.02$
Positive clinical response to therapy

defined as a decrease in the UCDAI score by $\geq 2$ points

\section{Butyrate:}

Clinical improvement (decrease in UCDAI score NS $\geq 2$ points)

Overall changes in UCDAI scores NS

Endoscopic scores NS

Histologic scores
Positive clinical response to therapy defined as a decrease in the UCDAI score by $\geq 3$ points

\section{SCFA and butyrate:}

Clinical improvement (decrease in UCDAI score NS $\geq 3$ points)

Overall change in UCDAI scores NS

Humoral parameters of inflammation NS

Endoscopic scores NS

Histologic scores NS

\section{Butyrate vs placebo:}

Fewer colonic segments affected

endoscopically 
Table 7.1. Continued

\begin{tabular}{|c|c|c|c|}
\hline Reference & No. of patients & Type of intervention and placebo & $\begin{array}{l}\text { Frequency and } \\
\text { duration of } \\
\text { treatment }\end{array}$ \\
\hline Scheppach et al. 189 & $\begin{array}{l}35 \text { patients with } \\
\text { active UC (9 SCFA; } 12 \\
\text { butyrate; } 14 \text { placebo) } \\
\text { Patients selected } \\
\text { from Scheppach et } \\
\text { al. }{ }^{188}\end{array}$ & $\begin{array}{l}\text { Treatment 1: } 60 \mathrm{~mL} \text { of SCFA (acetate } \\
\text { at } 60 \mathrm{mmol} / \mathrm{L} \text {, propionate at } \\
30 \mathrm{mmol} / \mathrm{L} \text {, butyrate at } 40 \mathrm{mmol} / \mathrm{L} \text { ) } \\
\text { Treatment 2: } 60 \mathrm{~mL} \text { of butyrate at } \\
100 \mathrm{mmol} / \mathrm{L} \\
\text { Placebo: } 60 \mathrm{~mL} \text { of isotonic } \mathrm{NaCl} \\
\text { Constant oral medication } \\
\text { (mesalazine, sulfasalazine, } \\
\text { prednisone) }\end{array}$ & Twice daily for 8 wk \\
\hline Breuer et al..$^{190}$ & $\begin{array}{l}91 \text { patients with } \\
\text { active UC ( } 45 \text { SCFA; } \\
46 \text { placebo) }\end{array}$ & $\begin{array}{l}\text { Treatment: } 100 \mathrm{~mL} \text { of SCFA } \\
\text { (acetate } 80 \mathrm{mmol} / \mathrm{L} \text {, propionate at } \\
30 \mathrm{mmol} / \mathrm{L} \text {, butyrate at } 40 \mathrm{mmol} / \mathrm{L} \text { ) } \\
\text { Placebo: } 100 \mathrm{~mL} \text { of isotonic } \mathrm{NaCl} \\
\text { Constant oral medication } \\
\text { (mesalazine, sulfasalazine) }\end{array}$ & Twice daily for $6 \mathrm{wk}$ \\
\hline Lührs et al. ${ }^{191}$ & $\begin{array}{l}11 \text { patients with } \\
\text { active UC ( } 6 \text { butyrate; } \\
5 \text { placebo) } \\
\text { Patients selected } \\
\text { from Scheppach et } \\
\text { al. }{ }^{188}\end{array}$ & $\begin{array}{l}\text { Treatment: } 60 \mathrm{~mL} \text { of butyrate at } \\
100 \mathrm{mmol} / \mathrm{L} \\
\text { Placebo: } 60 \mathrm{~mL} \text { of isotonic } \mathrm{NaCl} \\
\text { Constant oral medication } \\
\text { (mesalazine, sulfasalazine, } \\
\text { prednisone) }\end{array}$ & Twice daily for 8 wk \\
\hline
\end{tabular}

\begin{tabular}{|c|c|c|c|}
\hline Vernia et al. ${ }^{192}$ & $\begin{array}{l}51 \text { patients with } \\
\text { active mild to } \\
\text { moderate UC } \\
\text { refractory to } \\
\text { standard therapy for } \\
8 \text { wk } \\
\text { ( } 24 \text { treatment; } 27 \\
\text { placebo) }\end{array}$ & $\begin{array}{l}\text { Treatment: } 4 \mathrm{~g} \text { of topical mesalazine } \\
+80 \mathrm{~mL} \text { of butyrate at } 80 \mathrm{mmol} / \mathrm{L} \\
\text { Placebo: } 4 \mathrm{~g} \text { of topical mesalazine }+ \\
80 \mathrm{~mL} \text { of } \mathrm{NaCl}\end{array}$ & Twice daily for 6 wk \\
\hline
\end{tabular}

Abbreviations: $\mathrm{NaCl}$, sodium chloride; NF-kB, nuclear factor-kappa B; NS, not significant; SCFAs, short-chain fatty acids; UC, ulcerative colitis; UCDAl, ulcerative colitis daily activity score. 
Histologic changes (ulcerations,

Butyrate:

crypt abscesses, distortion of crypt

Reduced density of neutrophils in the lamina

0.07 architecture, changes in lamina propria, alterations in the surface, and crypt epithelium)

propria

\section{SCFAs and butyrate:}

Reduced number of proliferating cells in upper $\quad<0.05$ $40 \%$ of crypts

Disease remission or marked

improvement in clinical symptoms (stool

frequency, rectal bleeding, disease

activity index), endoscopic score (0-3),

and histologic score (0-12)

\section{SCFAs:}

Lower mucin depletion

0.03

Clinical activity index

NS

Endoscopic scores

NS

Histologic scores
Nuclear NF-kB translocation (i.e. activation)

\section{Butyrate:}

Decreased number of positive macrophages $\quad<0.001$ for nuclear NF-KB

Clinical improvement (decrease in UCDAI score 0.009 $\geq 3$ points)

Endoscopic scores $\quad 0.052$

Decreased number of neutrophils in crypt $\quad 0.044$

epithelium

Decreased number of neutrophils in surface $\quad 0.021$

epithelium

\section{Disease remission or improvement in}

clinical symptoms (stool frequency/ consistency, rectal bleeding, nocturnal bowel movement, urgency, tenesmus, abdominal pain, patient self-evaluation), endoscopic score (0-3), and histologic score (0-3)

\section{Butyrate enema + mesalazine:}

Improvement in combined clinical score $\quad<0.05$

Decrease in stool frequency $\quad<0.01$

Decrease in urgency/tenesmus $\quad<0.05$

Improved patient self-evaluation $\quad<0.01$

Endoscopic scores NS

Histologic scores NS 
Table 7.1. Continued

\begin{tabular}{|c|c|c|c|}
\hline Reference & No. of patients & Type of intervention and placebo & $\begin{array}{l}\text { Frequency and } \\
\text { duration of } \\
\text { treatment }\end{array}$ \\
\hline Hamer et al. ${ }^{67}$ & $\begin{array}{l}35 \text { patients with } \\
\text { UC in remission ( } 17 \\
\text { butyrate; } 18 \text { placebo) }\end{array}$ & $\begin{array}{l}\text { Treatment: } 60 \mathrm{~mL} \text { of butyrate at } \\
100 \mathrm{mmol} / \mathrm{L} \\
\text { Placebo: } 60 \mathrm{~mL} \text { of } \mathrm{NaCl} \\
\text { Constant oral medication } \\
\text { (mesalazine, sulfasalazine, } \\
\text { azathioprine) }\end{array}$ & $\begin{array}{l}\text { One enema nightly } \\
\text { for } 20 \mathrm{~d}\end{array}$ \\
\hline
\end{tabular}

Abbreviations: NaCl, sodium chloride; NF-KB, nuclear factor-kappa B; NS, not significant; SCFAs, short-chain fatty acids; UC, ulcerative colitis; UCDAl, ulcerative colitis daily activity score. 
Primary endpoint

Inflammation and oxidative stress

\section{Results}

$P$-value

\section{Butyrate:}

Increase in chemokine CCL5 in mucosal

0.029

biopsies

Downregulation of xanthine dehydrogenase in $\quad<0.05$

mucosal biopsies

Daily symptom score

NS

Other humoral and mucosal inflammatory

NS

parameters

Oxidative stress 
Fiber supplementation in patients with ulcerative colitis is not widely tested, ${ }^{195}$ especially not in relation to changes in fecal SCFA concentrations. An open-label, randomized, controlled multicenter trial was conducted to investigate the efficacy and safety of fermentable dietary fiber (Plantago ovata seeds) vs mesalazine to maintain remission in patients with inactive ulcerative colitis. There was a significant increase in butyrate concentrations and a trend toward increasing acetate and total SCFA concentrations in feces after 3 months of fiber intake. The results failed to show a difference between the treatments in preventing relapse, suggesting that fermentable fiber might be as effective as mesalazine for maintaining remission. ${ }^{196}$ More clinical trials should be conducted on this subject.

Overall, although most studies point toward a positive role of SCFAs or fiber supplementation in the treatment of ulcerative colitis, no convincing evidence in placebo-controlled trials has been found. It appears that SCFA agonism is not a sufficient anti-inflammatory stimulus to resolve inflammatory colitis, since the most promising treatment seems to be a combination of SCFAs and other anti-inflammatory agents, such as mesalazine.

\section{Diversion colitis}

Diversion colitis can occur after surgical diversion of the fecal stream and may present, for instance, after a Hartmann procedure with closure of the rectum or sigmoid. In a considerable number of patients, the excluded part of the colon will develop moderate to severe inflammation. It has been assumed that lack of luminal SCFAs causes a metabolic deficiency, contributing to inflammation. ${ }^{116,197}$ The abatement of colitis when the fecal stream is restored supports this assumption. ${ }^{198}$ The effects of SCFA treatment in diversion colitis were first studied by Harig et al. ${ }^{199}$, who showed clinical and endoscopic improvement in 4 patients after SCFA application in the excluded rectosigmoid. A placebo enema of isotonic saline administered during the regimen did not reduce the inflammation. A beneficial effect of SCFA treatment was also found in 5 children with refractory proctocolitis, ${ }^{200} 9$ children with acute diversion proctocolitis, ${ }^{201}$ and 4 adult patients. ${ }^{202}$ However, when investigated in a placebo-controlled manner, these effects could not be confirmed (Table 7.2). ${ }^{203-205}$ Guillemot et al. ${ }^{203}$ and Schauber et al. ${ }^{204}$ investigated the effect of a SCFA mixture vs placebo in 13 and 9 patients, respectively. Neither study detected any difference between treatments in patient self-evaluation, endoscopic score, or histologic score. A recent placebo-controlled study by Luceri et al. ${ }^{205}$, however, showed significantly improved endoscopic grading after a high amount of butyrate $(600 \mathrm{mmol} / \mathrm{L})$ was administered rectally, with no adverse events. In conclusion, the current literature does not convincingly support the benefit of SCFAs in the treatment of diversion colitis, but recent studies point towards improvement of the disease after SCFA treatment. 


\section{Radiation proctitis}

Radiation proctitis occurs in about $25 \%$ of patients receiving radiation therapy for pelvic cancer. It causes severe and debilitating symptoms such as bleeding, pain, and increased stool frequency. ${ }^{206}$ The promising effects of SCFAs in other inflammatory diseases prompted the investigation of the effect of SCFAs in the treatment of radiation proctitis. To date, 7 studies have examined the use of SCFAs in the treatment of radiation proctitis. ${ }^{207-213}$ Only 4 of these are randomized, placebo-controlled studies, the rest being open or pilot studies (Table 7.3). ${ }^{209-211,213}$

In acute radiation proctitis, an overnight butyrate enema for 3 weeks resulted in marked improvement in clinical markers such as stool consistency, rectal blood discharge, and rectal burning or tenesmus as well as a higher patient self-evaluation score when compared with placebo treatment. ${ }^{211}$ Endoscopic scores, graded from 0 (normal mucosal pattern) to 3 (mucosal alterations, spontaneous bleeding), and histologic scores, graded from 0 (normal mucosa) to 3 (severe inflammation/damage), tended to differ between the butyrate group and the placebo group. This study included 20 patients, 10 in the treatment arm and 10 in the placebo arm, which is a rather small number. It was originally designed as a randomized crossover trial, but since a carryover effect from the first study period to the second was high, only results from the first study period were reported.

Chronic radiation proctitis was examined in 2 placebo-controlled studies. The first, by Talley et al. ${ }^{209}$, was a randomized crossover trial in which 12 patients with chronic radiation proctitis received butyrate twice daily for 2 weeks. No significant effects on clinical, endoscopic, or histologic scores were found when the treatment arm was compared with the placebo arm. In the second study, by Pinto et al.210, 16 patients with chronic radiation proctitis were randomized to receive either a SCFA mixture or placebo, twice daily for 5 weeks. Patients were monitored for 6 months after the enemas were ceased. When compared with placebo, the butyrate enemas had a direct effect on the hemoglobin concentration, endoscopic score, and histologic fibrosis after the 5-week treatment. One month after the enemas were ceased, there was still a difference between the butyrate and placebo groups in rectal bleeding and the endoscopic score, but this difference was no longer significant 6 months after the enemas ended.

In the fourth placebo-controlled study, the aim was to prevent proctitis in 166 patients undergoing radiation therapy by providing butyrate enemas during the therapy. ${ }^{213}$ The patients were divided into 4 groups: 1 group received a placebo, and the other 3 groups received a gradually increasing dose of butyrate ( $1 \mathrm{~g}, 2 \mathrm{~g}$, or $4 \mathrm{~g}$ ). The authors found no effect of the different amounts of butyrate on the occurrence of proctitis, the duration of proctitis, or the endoscopic scores when comparing butyrate treatment and placebo. They did, however, report that the study might have been underpowered, since the incidence of proctitis was calculated as $60 \%$, while the true incidence was $40 \%$. 
Table 7.2. Clinical placebo-controlled studies evaluating the effect of rectal treatment with short-chain fatty acids in diversion colitis

\begin{tabular}{|c|c|c|c|}
\hline Reference & No. of patients & Type of intervention and placebo & $\begin{array}{l}\text { Frequency and } \\
\text { duration of } \\
\text { treatment }\end{array}$ \\
\hline Guillemot et al. ${ }^{203}$ & $\begin{array}{l}13 \text { patients with } \\
\text { diversion colitis } \\
\text { (7 SCFAs; } 6 \text { placebo) }\end{array}$ & $\begin{array}{l}\text { Treatment: } 60 \mathrm{~mL} \text { of SCFAs (acetate } \\
\text { at } 60 \mathrm{mmol} / \mathrm{L} \text {, propionate at } \\
30 \mathrm{mmol} / \mathrm{L} \text {, butyrate at } 40 \mathrm{mmol} / \mathrm{L} \text { ) } \\
\text { Placebo: } 60 \mathrm{~mL} \text { of isotonic } \mathrm{NaCl}\end{array}$ & Twice daily for 2 wk \\
\hline Schauber et al. ${ }^{204}$ & $\begin{array}{l}9 \text { patients with } \\
\text { refractory diversion } \\
\text { colitis } \\
\text { Randomized } \\
\text { crossover design }\end{array}$ & $\begin{array}{l}\text { Treatment: } 60 \mathrm{~mL} \text { of SCFAs (acetate } \\
\text { at } 80 \mathrm{mmol} / \mathrm{L} \text {, propionate at } \\
30 \mathrm{mmol} / \mathrm{L} \text {, butyrate at } 40 \mathrm{mmol} / \mathrm{L} \text { ) } \\
\text { Placebo: } 60 \mathrm{~mL} \text { of isotonic } \mathrm{NaCl}\end{array}$ & $\begin{array}{l}\text { Twice daily for } \\
3 \text { wk, followed } \\
\text { directly by a } \\
\text { second study } \\
\text { period of } 3 \text { wk }\end{array}$ \\
\hline Luceri et al. ${ }^{205}$ & $\begin{array}{l}17 \text { patients with } \\
\text { enterostomy for } \\
\text { IBD, cancer, or } \\
\text { diverticulitis } \\
\text { (10 butyrate; } \\
7 \text { placebo) }\end{array}$ & $\begin{array}{l}\text { Treatment: } 30 \mathrm{~mL} \text { of butyrate at } \\
600 \mathrm{mmol} / \mathrm{L} \\
\text { Placebo: } 30 \mathrm{~mL} \text { of isotonic } \mathrm{NaCl} \text { with } \\
3 \mathrm{mmol} / \mathrm{L} \text { butyrate }\end{array}$ & Twice daily for $30 \mathrm{~d}$ \\
\hline
\end{tabular}

Abbreviations: IBD, inflammatory bowel disease; $\mathrm{NaCl}$, sodium chloride; NS, not significant; SCFAs, short-

chain fatty acids. 


\section{Endoscopic score (0-10) and histologic} score (0-10)

\section{SCFAs:}

Endoscopic scores

NS

Histologic scores

\section{Improvement in clinical symptoms (rectal SCFAs:}

bleeding, patient and physician combined evaluation), endoscopic score (0-10), and histologic score (0-6), rectal swab

Global assessment by patient and physician Endoscopic scores

Histologic scores Bacterial content

Safety of butyrate at high concentrations, efficacy in improving endoscopic and histologic scores

\section{Butyrate:}

Reduced endoscopic grading

Histopathologic grading
NS NS

NS

NS

0.008

NS 
Table 7.3. Clinical placebo-controlled studies evaluating the effect of rectal SCFA treatment in radiation proctitis

\begin{tabular}{|c|c|c|c|}
\hline Reference & No. of patients & Type of intervention and placebo & $\begin{array}{l}\text { Frequency and } \\
\text { duration of } \\
\text { treatment }\end{array}$ \\
\hline Talley et al. ${ }^{209}$ & $\begin{array}{l}12 \text { patients with } \\
\text { chronic radiation } \\
\text { proctitis (randomized } \\
\text { crossover design) }\end{array}$ & $\begin{array}{l}\text { Treatment: } 60 \mathrm{~mL} \text { of butyrate at } \\
40 \mathrm{mmol} \\
\text { Placebo: } 60 \mathrm{~mL} \text { of } 0.9 \% \mathrm{NaCl}\end{array}$ & $\begin{array}{l}\text { Twice daily for } 2 \text { wk } \\
\text { 1-wk washout } \\
\text { period before } \\
\text { alternate enema } \\
\text { treatment }\end{array}$ \\
\hline Pinto et al. ${ }^{210}$ & $\begin{array}{l}16 \text { patients with } \\
\text { chronic radiation } \\
\text { proctitis ( } 9 \text { SCFAs; } 7 \\
\text { placebo) }\end{array}$ & $\begin{array}{l}\text { Treatment: } 60 \mathrm{~mL} \text { of SCFA (acetate } \\
\text { at } 60 \mathrm{mmol} / \mathrm{L} \text {, propionate at } \\
30 \mathrm{mmol} / \mathrm{L} \text {, butyrate at } 40 \mathrm{mmol} / \mathrm{L} \text { ) } \\
\text { Placebo: } 60 \mathrm{~mL} \text { of isotonic } \mathrm{NaCl}\end{array}$ & $\begin{array}{l}\text { Twice daily for } 5 \text { wk } \\
\text { Follow-up for } 1 \text { mo } \\
\text { and } 6 \text { mo after } \\
\text { treatment was } \\
\text { stopped }\end{array}$ \\
\hline Vernia et al. ${ }^{211}$ & $\begin{array}{l}20 \text { patients with } \\
\text { acute radiation } \\
\text { proctitis ( } 10 \text { butyrate; } \\
10 \text { placebo) }\end{array}$ & $\begin{array}{l}\text { Treatment: } 80 \mathrm{~mL} \text { of butyrate at } \\
80 \mathrm{mmol} / \mathrm{L} \\
\text { Placebo: } 80 \mathrm{~mL} \text { of } \mathrm{NaCl}+1 \text { drop of } \\
\text { butyrate }\end{array}$ & $\begin{array}{l}\text { One enema nightly } \\
\text { for } 3 \text { wk }\end{array}$ \\
\hline Maggio et al. ${ }^{213}$ & $\begin{array}{l}\text { Prevention of } \\
\text { proctitis: } 166 \\
\text { patients undergoing } \\
\text { radiation for } \\
\text { prostate cancer ( } 38 \\
\text { placebo; } 40,40 \text {, and } \\
40 \text { each treatment) }\end{array}$ & $\begin{array}{l}\text { Treatment 1: } 1 \mathrm{~g} \text { of butyrate as } \\
\text { sodium butyrate solution } \\
\text { Treatment 2: } 2 \mathrm{~g} \text { of butyrate as } \\
\text { sodium butyrate solution } \\
\text { Treatment } 3: 4 \mathrm{~g} \text { of butyrate as } \\
\text { sodium butyrate solution } \\
\text { Placebo: unknown }\end{array}$ & $\begin{array}{l}\text { Twice daily for } \\
13 \text { wk } \\
\text { (entire course of } \\
\text { radiotherapy }+2 \\
\text { additional weeks) }\end{array}$ \\
\hline
\end{tabular}

Abbreviations: Gl, gastrointestinal; IBD, inflammatory bowel disease; $\mathrm{NaCl}$, sodium chloride; NS, not significant; SCFAs, short-chain fatty acids. 
Marked improvement in clinical

symptoms (rectal pain, rectal bleeding, urgency, diarrhea, stool movements), endoscopic score (0-8), and histologic score (0 or 1$)$

Marked improvement in clinical symptoms (rectal bleeding, hemoglobin concentration), endoscopic score (0-10), and histologic score (0-2)

\section{Butyrate:}

Symptom scores NS

Endoscopic scores NS

Histologic scores NS

\section{Acute effect of SCFAs (5-wk):}

Mean days with rectal bleeding

0.13

Increased hemoglobin concentration $\quad 0.02$

Endoscopic scores $\quad 0.02$

Presence fibrosis mucosal biopsies $\quad 0.02$

Long-term effect of SCFAs (1 mo):

Mean days with rectal bleeding $\quad 0.06$

Endoscopic scores $\quad 0.01$

Long-term effect of SCFAs (6 mo):

Mean days with rectal bleeding NS

Endoscopic scores

NS

Marked improvement in clinical
symptoms (stool consistency, rectal
bleeding, abdominal pain, tenesmus, self-
evaluation), endoscopic score (0-3), and
histologic score (0-3)

\section{Butyrate:}

Overall clinical scores $\quad<0.05$

Improved stool consistency $\quad 0.005$

Decrease in bloody stools 0.01

Decrease in burning/tenesmus $\quad 0.04$

Improved self-evaluation $\quad 0.01$

Endoscopic scores $\quad 0.05$

Histologic inflammation $\quad 0.06$

Decrease in bowel movements $\quad 0.008$

Decrease in nocturnal bowel movements $\quad 0.0006$

Prevention of acute proctitis and efficacy

of sodium butyrate in reducing of the

Butyrate:

Presence of proctitis NS

Duration of proctitis NS

Endoscopic scores for Gl damage NS 
Overall, SCFA treatment seems to affect the acute phase of radiation proctitis, possibly by speeding the healing process. However, it might not help prevent the disease or the chronic form of it. The lack of an effect on chronic proctitis might be explained by a different mechanism in acute versus chronic proctitis. Acute proctitis is due mainly to acute mucosal injury and inflammation involving various cellular and cytokine pathways that can be influenced by SCFA treatment. Chronic proctitis involves an underlying chronic process of fibrosis and vascular sclerosis, which is not reversible. ${ }^{214}$

\section{Pouchitis}

In patients with severe colitis unresponsive to medical interventions, total colectomy with creation of an ileo-anal pouch is considered. This pouch may, over time, show signs of mucosal inflammation, a condition called pouchitis. Only one study has investigated SCFA treatment in the pouch of ulcerative colitis patients. The authors showed that SCFA administration decreased the proliferative activity of the mucosa in patients with a pouch, but no effects on endoscopic appearance of the pouch, histopathologic parameters of inflammation, or daily symptoms were observed. ${ }^{215}$ Another study investigated the effects of dietary fiber administration in patients with an ileal pouch-anal anastomosis. This placebo-controlled, randomized, double-blind, crossover study revealed that 3 weeks of inulin ingestion resulted in decreased intestinal mucosal inflammation (at histologic and endoscopic levels) as well as changes in the microbiota, increased fecal butyrate concentrations, and decreased secondary bile acids. ${ }^{216}$

\section{Crohn's disease}

Crohn's disease is a type of inflammatory bowel disease that may present throughout the whole gastrointestinal tract, extending deep into the bowel wall. This makes treatment with topical agents very difficult. To the best of knowledge, only one uncontrolled study providing SCFAs has been performed in patients with Crohn's disease. ${ }^{217}$ In that study, 13 patients with mild to moderate Crohn's disease received butyrate twice daily for 8 weeks via enteric-coated tablets designed to release the butyrate in the terminal ileum and colon. Nine patients responded to treatment, of whom 7 had a complete response and 2 a partial response in disease activity as assessed by the Crohn's Disease Activity Index, a research tool used to quantify the symptoms of patients with Crohn's disease. The endoscopic and histologic scores of the patients improved after treatment, and NF-KB and IL-1 $\beta$ protein levels in mucosal biopsies from the terminal ileum had decreased. These results seem promising, but since a placebo effect cannot be excluded, they need to be confirmed by larger placebo-controlled studies. 


\section{CLINICAL HUMAN INTERVENTION TRIALS: COLON CARCINOGENESIS}

The role of SCFAs in colon carcinogenesis has not been investigated extensively in human trials. Two studies in ulcerative colitis patients treated with nocturnal enemas containing either butyrate or a mixture of SCFAs showed a $40 \%$ reduction of proliferating crypt cells in treated patients compared with those who received placebo, indicating that SCFAs may prevent the development of colon cancer in the inflamed state. ${ }^{185,189}$ In healthy humans, however, it is not feasible to apply SCFAs directly into the colon for several years or decades in order to prevent colorectal cancer. Therefore, the intake of fiber is more logical to investigate in a human intervention study. Beyond that, the use of prodrugs, such as tributyrin, would enable an already developed tumor located anywhere in the colon to be reached, and not only tumors in the distal part, which can be reached using enemas.

Le Leu et al. ${ }^{218}$ recently investigated whether an increased risk of developing colorectal cancer after intake of red meat, considered as carcinogenic, could be prevented by adding fiber to the diet. In a randomized controlled study, 10 healthy volunteers received a diet high in red meat for 4 weeks, while 13 healthy volunteers ingested the same diet along with $40 \mathrm{~g}$ of butyrylated high-amylose maize starch. The authors showed that intake of red meat increased toxic compounds and rectal epithelial proliferation in rectal biopsies, which was counteracted by the addition of butyrylated starch to the diet. A higher excretion of acetate, propionate, and butyrate in the stool of the starch group was found when compared with baseline values. The results imply that dietary fiber protects the gut against toxic compounds and carcinogenic alterations, possibly via fermentation of fiber into SCFAs. Other studies in healthy volunteers 219,220 and in colorectal cancer patients 221 have confirmed this anti-carcinogenic effect of resistant starch, indicating possible protection through SCFA production.

On the other hand, a large multicenter randomized controlled trial in 714 patients with Lynch syndrome (hereditary nonpolyposis colorectal cancer) who were predisposed to develop colon cancer showed no effect of long-term intake of resistant starch on cancer development.222 In that study, however, the dose of starch and, thus, the amount of available SCFAs were much lower than in the trial by Le Leu et al.218. Furthermore, the development of hereditary colorectal cancer might involve mechanisms other than those targeted by SCFAs. Le Leu et al. ${ }^{218}$ also used a highly toxigenic diet in which the butyrylated starch counteracted the toxic effects of, for example, red meat, while the Lynch patients consumed a normal diet. It is possible that some markers might show changes at an early stage of colon cancer, e.g., the proliferation markers used by Le Leu et al. ${ }^{218}$, while other markers show changes at a later stage of colon cancer development, such as those used in the Lynch study. In conclusion, these 2 studies do not provide a definitive answer. 
Although the role of dietary fiber in relation to colorectal cancer has been investigated for many years, recent original studies continue to provide new insights. O'Keefe et al.223 conducted a 2-week dietary intervention trial in native Africans, who have a low rate of colorectal cancer, and African Americans, who have a 10-fold higher rate of colorectal cancer. Native African participants switched from their traditional high-fiber diets to lowfiber Western diets, and the American participants switched to the fiber-rich diet. The dietary changes affected the gut microbiome and led to profound changes in metabolites. An increase in saccharolytic fermentation and butyrogenesis as well as suppression of secondary bile acid synthesis was observed in African American participants after switching to a high-fiber, low-fat diet. These changes were associated with a significant reduction in both colonic mucosal inflammation and biomarkers of cell proliferation. In sharp contrast, the diet switch for 2 weeks to a high-fat, low-fiber diet in rural African participants resulted in reverse changes in all these parameters. ${ }^{223}$ It must be noted however, that these findings need to be confirmed in longitudinal studies, as there is still controversy over the effects of dietary fiber on colorectal cancer. Indeed, a large pooled analysis of data from 13 prospective cohort studies failed to show a linear inverse association between dietary fiber intake and risk of colorectal cancer. ${ }^{224}$ Clearly, the amount of dietary fiber consumed per day may influence outcome. Rather high doses of more than $50 \mathrm{~g} / \mathrm{d}$ (as in the study of O'Keefe et al. $^{223}$ ), as well as a microbiome that is able to generate sufficient amounts of butyrate, 139 may be needed to observe a protective effect against colorectal cancer. In addition, dietary interactions may play a role. For example, it was recently shown that a vegetarian diet, in particular a pesco-vegetarian diet, was associated with a lower risk of colorectal cancer. 225 Potential underlying mechanisms of such dietary interactions have been recently reviewed. ${ }^{226}$

Prodrugs offer the possibility to treat already developed tumorous tissue. Since trials of these medications are under strict rules and are limited to severely diseased populations, not many have been conducted. In several human clinical trials on prodrugs in patients with various types of cancers, including colon cancer, prostate cancer, non-small cell lung cancer, and resistant myeloid neoplasm, only limited improvement or stabilization of disease was described. ${ }^{227-233}$ A limitation of these phase I pharmacological studies is that they are primarily designed to evaluate the toxicity and maximum or optimal dose of the prodrug. For ethical reasons, only certain tumors refractory to standard therapy were investigated, and prodrug therapy was not targeted at colon cancer specifically. The field of prodrug treatment is still developing, so studies targeted specifically at colon cancer might be conducted in the future. 


\section{CLINICAL HUMAN INTERVENTION TRIALS: MUCOSAL HEALING AND THE INTESTINAL BARRIER}

Prior to major colonic surgery, the bowel is cleansed of feces to avoid anastomotic leaks and secondary infections by bacterial content. However, several studies have shown that bowel preparation does not contribute to better anastomotic healing or the prevention of anastomotic leakage. ${ }^{234-236}$ This lack of effect might be attributable to lower SCFA concentrations in the cleansed colon. As far as can be determined, however, no human studies have been conducted to assess the effects of SCFAs on mucosal healing. Several studies have, however, attempted to improve the protective characteristic of the intestinal barrier. In healthy volunteers, an improved intestinal barrier was observed after chronic intake of inulin, a prebiotic known to increase colonic SCFA concentrations. ${ }^{237}$ Mucus secretion, however, as measured by the amount of mucins present in $24 \mathrm{~h}$ feces, and MUC2 expression did not differ after rectal butyrate infusions in ulcerative colitis patients in remission compared with placebo or with butyrate treatment in healthy volunteers. ${ }^{238}$ Hamer et al. ${ }^{239}$ reported that butyrate enemas increased the antioxidant status in human healthy volunteers. In ulcerative colitis patients, however, the same authors found no effect on antioxidant status. ${ }^{67}$ Some intervention studies have tried to gain insight into cell proliferation rates after SCFA application in humans, showing that mucosal cell proliferation and mucosal blood flow is enhanced after SCFA application in a closed Hartmann's rectum. 37,240 These studies, however, are translational and do not give direct insight into the possible role of SCFAs in mucosal healing. 


\section{FUTURE PERSPECTIVES}

Overall, clinical trials on SCFA application have not confirmed the promise of preclinical trials. The higher doses of SCFAs used in animal studies might explain the lack of effect in humans. Another factor, as already noted, is that patients investigated in clinical trials might be refractory to established medication and thereby have far more complicated disease than can be mimicked in animal models. Clinical research is further complicated by the fact that each individual has a specific microbiota composition. This review underlines the importance of expanding research into human investigational studies.

In clinical practice, the colonic application of SCFAs is not yet an established treatment option for colonic disease in humans. Especially in inflammatory bowel disease, the results from human intervention studies are disappointing. The limited effects of SCFA application in inflammatory bowel disease are in line with a recent meta-analysis showing no effect of fiber intake on inflammation. ${ }^{241}$ However, the possibility to combine SCFAs with antiinflammatory drugs such as mesalazine seems promising. ${ }^{194}$ In addition, the combination of SCFAs with probiotics or prebiotics, ${ }^{110}$ or even the combination of butyrate with a prebiotic plus mesalazine, ${ }^{242}$ might have a role in further optimizing the treatment of inflammatory bowel disease. As for the prevention of colon cancer, larger, prospective studies on the use of butyrylated fibers could be conducted to validate the promising effects found in animal studies. Likewise, such studies should be performed to investigate the use of location-specific SCFA prodrugs in the treatment of colon cancer. Animal studies on anastomotic healing are promising, so the application of SCFAs in humans undergoing colonic surgery is another area that warrants research. In the future, butyrate-coated sutures might aid mucosal healing after colonic surgery, as hypothesized from a study that showed better tendon healing when butyric acid-impregnated sutures were used in rats. ${ }^{243}$

\section{CONCLUSION}

The use of SCFAs, especially butyrate but also acetate and propionate, to treat colonic diseases has shown promising results in vitro and ex vivo. Viewing data from current research, it seems not likely, however, that SCFA treatment as a stand-alone therapy will prove effective in treating or preventing colonic diseases. In the future, the use of SCFAs combined with anti-inflammatory drugs, prebiotics, or probiotics, the use of prodrugs in the case of carcinogenesis, or the direct application of SCFAs using SCFA-coated accessories to improve mucosal healing after colonic surgery may provide new therapeutic options. 


\section{REFERENCES}

1. Nagalingam NA, Lynch SV. Role of the microbiota in inflammatory bowel diseases. Inflamm Bowel Dis. 2012;18:968-984.

2. Topping DL, Clifton PM. Short-chain fatty acids and human colonic function: roles of resistant starch and nonstarch polysaccharides. Physiol Rev. 2001;81:1031-1064.

3. Wong JM, de Souza R, Kendall CW, et al. Colonic health: fermentation and short chain fatty acids. J Clin Gastroenterol. 2006;40:235-243.

4. Bergman EN. Energy contributions of volatile fatty acids from the gastrointestinal tract in various species. Physiol Rev. 1990;70:567-590.

5. Hamer HM, Jonkers $\mathrm{D}$, Venema $\mathrm{K}$, et al. Review article: the role of butyrate on colonic function. Aliment Pharmacol Ther. 2008;27:104-119.

6. Canani RB, Costanzo MD, Leone L, et al. Potential beneficial effects of butyrate in intestinal and extraintestinal diseases. World J Gastroenterol. 2011;17:1519-1528.

7. Koh A, De Vadder F, Kovatcheva-Datchary P, et al. From dietary fiber to host physiology: short-chain fatty acids as key bacterial metabolites. Cell. 2016;165:1332-1345.

8. Boets E, Gomand SV, Deroover L, et al. Systemic availability and metabolism of colonic-derived short-chain fatty acids in healthy subjects: a stable isotope study. J Physiol. 2017:595:541-555.

9. Wolever TM, Spadafora $\mathrm{P}$, Eshuis $\mathrm{H}$. Interaction between colonic acetate and propionate in humans. Am J Clin Nutr. 1991;53:681-687.

10. den Besten G, Lange K, Havinga R, et al. Gut-derived short-chain fatty acids are vividly assimilated into host carbohydrates and lipids. Am J Physiol Gastrointest Liver Physiol. 2013;305:G900-G910.

11. Cummings JH, Pomare EW, Branch WJ, et al. Short chain fatty acids in human large intestine, portal, hepatic and venous blood. Gut. 1987;28:1221-1227.

12. Ahmed R, Segal I, Hassan H. Fermentation of dietary starch in humans. Am J Gastroenterol. 2000;95:1017-1020.

13. Cummings JH, Macfarlane GT. Colonic microflora: nutrition and health. Nutrition. 1997;13:476-478.

14. Argenzio RA, Southworth M. Sites of organic acid production and absorption in gastrointestinal tract of the pig. Am J Physiol. 1975;228:454-460.

15. Vogt JA, Wolever TM. Fecal acetate is inversely related to acetate absorption from the human rectum and distal colon. J Nutr. 2003;133:3145-3148.

16. van der Beek CM, Bloemen JG, van den Broek MA, et al. Hepatic uptake of rectally administered butyrate prevents an increase in systemic butyrate concentrations in humans. J Nutr. 2015;145:2019-2024.

17. Bloemen JG, Venema K, van de Poll MC, et al. Short chain fatty acids exchange across the gut and liver in humans measured at surgery. Clin Nutr. 2009;28:657-661.

18. Cummings JH. Colonic absorption: the importance of short chain fatty acids in man. Scand J Gastroenterol Suppl. 1984;93:89-99.

19. Halestrap AP, Meredith D. The SLC16 gene family - from monocarboxylate transporters (MCTs) to aromatic amino acid transporters and beyond. Pflugers Arch. 2004;447:619-628.

20. Harig JM, Ng EK, Dudeja PK, et al. Transport of n-butyrate into human colonic luminal membrane vesicles. Am J Physiol. 1996;271(3, pt 1):G415-G422. 
21. Miyauchi S, Gopal E, Fei YJ, et al. Functional identification of SLC5A8, a tumor suppressor down-regulated in colon cancer, as a $\mathrm{Na}+$-coupled transporter for short-chain fatty acids. J Biol Chem. 2004;279:13293-13296.

22. Tyagi S, Venugopalakrishnan J, Ramaswamy K, et al. Mechanism of n-butyrate uptake in the human proximal colonic basolateral membranes. Am J Physiol Gastrointest Liver Physiol. 2002;282:G676-G682.

23. Gill RK, Saksena S, Alrefai WA, et al. Expression and membrane localization of MCT isoforms along the length of the human intestine. Am J Physiol Cell Physiol. 2005;289:C846-C852.

24. Brown AJ, Goldsworthy SM, Barnes AA, et al. The orphan G protein-coupled receptors GPR41 and GPR43 are activated by propionate and other short chain carboxylic acids. J Biol Chem. 2003;278:11312-11319.

25. Le Poul E, Loison C, Struyf S, et al. Functional characterization of human receptors for short chain fatty acids and their role in polymorphonuclear cell activation. J Biol Chem. 2003;278:25481-25489.

26. Tazoe H, Otomo Y, Kaji I, et al. Roles of short-chain fatty acids receptors, GPR41 and GPR43 on colonic functions. J Physiol Pharmacol. 2008;59(suppl 2):251-262.

27. Tazoe H, Otomo Y, Karaki S, et al. Expression of short-chain fatty acid receptor GPR41 in the human colon. Biomed Res. 2009;30:149-156.

28. Kim MH, Kang SG, Park JH, et al. Short-chain fatty acids activate GPR41 and GPR43 on intestinal epithelial cells to promote inflammatory responses in mice. Gastroenterology. 2013;145:396-406.

29. Thangaraju M, Cresci GA, Liu K, et al. GPR109A is a G-protein-coupled receptor for the bacterial fermentation product butyrate and functions as a tumor suppressor in colon. Cancer Res. 2009;69:2826-2832.

30. Singh N, Gurav A, Sivaprakasam S, et al. Activation of Gpr109a, receptor for niacin and the commensal metabolite butyrate, suppresses colonic inflammation and carcinogenesis. Immunity. 2014;40:128-139.

31. Sterner R, Vidali G, Allfrey VG. Studies of acetylation and deacetylation in high mobility group proteins. Identification of the sites of acetylation in high mobility group proteins 14 and 17 . J Biol Chem. 1981;256:8892-8895.

32. Clausen MR, Mortensen PB. Kinetic studies on colonocyte metabolism of short chain fatty acids and glucose in ulcerative colitis. Gut. 1995;37:684-689.

33. Hague A, Elder DJ, Hicks DJ, et al. Apoptosis in colorectal tumour cells: induction by the short chain fatty acids butyrate, propionate and acetate and by the bile salt deoxycholate. Int J Cancer. 1995;60:400-406.

34. Comalada M, Bailon E, de Haro $\mathrm{O}$, et al. The effects of short-chain fatty acids on colon epithelial proliferation and survival depend on the cellular phenotype. J Cancer Res Clin Oncol. 2006;132:487-497.

35. Roediger WE. The colonic epithelium in ulcerative colitis: an energy-deficiency disease? Lancet. 1980;316:712-715.

36. Mortensen FV, Nielsen H, Mulvany MJ, et al. Short chain fatty acids dilate isolated human colonic resistance arteries. Gut. 1990;31:1391-1394.

37. Mortensen FV, Hessov I, Birke $H$, et al. Microcirculatory and trophic effects of short chain fatty acids in the human rectum after Hartmann's procedure. Br J Surg. 1991;78:1208-1211.

38. Kvietys PR, Granger DN. Effect of volatile fatty acids on blood flow and oxygen uptake by the dog colon. Gastroenterology. 1981;80(5 pt 1):962-969. 
39. Donohoe DR, Garge N, Zhang X, et al. The microbiome and butyrate regulate energy metabolism and autophagy in the mammalian colon. Cell Metabol. 2011;13:517-526.

40. Elamin EE, Masclee AA, Dekker J, et al. Short-chain fatty acids activate AMP-activated protein kinase and ameliorate ethanol-induced intestinal barrier dysfunction in Caco-2 cell monolayers. J Nutr. 2013;143:1872-1881.

41. Peng L, Li ZR, Green RS, et al. Butyrate enhances the intestinal barrier by facilitating tight junction assembly via activation of AMP-activated protein kinase in Caco-2 cell monolayers. J Nutr. 2009;139:1619-1625.

42. Peng $\mathrm{L}, \mathrm{He} \mathrm{Z}$, Chen $\mathrm{W}$, et al. Effects of butyrate on intestinal barrier function in a Caco-2 cell monolayer model of intestinal barrier. Pediatr Res. 2007;61:37-41.

43. Suzuki T, Yoshida S, Hara H. Physiological concentrations of short-chain fatty acids immediately suppress colonic epithelial permeability. Br I Nutr. 2008;100:297-305.

44. Correa-Oliveira R, Fachi JL, Vieira A, et al. Regulation of immune cell function by short-chain fatty acids. Clin Transl Immunol. 2016;5:e73. doi:10.1038/cti.2016.17.

45. Terzi C, Sevinc Al, Kocdor H, et al. Improvement of colonic healing by preoperative rectal irrigation with short-chain fatty acids in rats given radiotherapy. Dis Colon Rectum. 2004;47:2184-2194.

46. Kripke SA, Fox AD, Berman JM, et al. Stimulation of intestinal mucosal growth with intracolonic infusion of short-chain fatty acids. JPEN J Parenter Enteral Nutr. 1989;13:109-116.

47. Wilson AJ, Gibson PR. Short-chain fatty acids promote the migration of colonic epithelial cells in vitro. Gastroenterology. 1997;113:487-496.

48. Aguilar-Nascimento JE, Franca-da-Silva LR, De-Oliveira AF, et al. Enhanced mucosal re-epithelialization induced by short chain fatty acids in experimental colitis. Braz J Med Biol Res. 1999;32:961-966.

49. Sakata T. Effects of indigestible dietary bulk and short chain fatty acids on the tissue weight and epithelial cell proliferation rate of the digestive tract in rats. J Nutr Sci Vitaminol (Tokyo). 1986;32:355-362.

50. Aguilar-Nascimento JE, Oliveira-Neto JP, Mathie RT, et al. Effect of intraoperative nutritional solutions on perianastomotic colonic mucosa in experimental large bowel obstruction. Dig Dis Sci. 1997;42:2581-2584.

51. Dhawan P, Singh AB, Deane NG, et al. Claudin-1 regulates cellular transformation and metastatic behavior in colon cancer. J Clin Invest. 2005;115:1765-1776.

52. Gecse K, Roka R, Sera T, et al. Leaky gut in patients with diarrhea-predominant irritable bowel syndrome and inactive ulcerative colitis. Digestion. 2012;85:40-46.

53. Den Hond E, Hiele M, Evenepoel P, et al. In vivo butyrate metabolism and colonic permeability in extensive ulcerative colitis. Gastroenterology. 1998;115:584-590.

54. Burger-van Paassen N, Vincent A, Puiman PJ, et al. The regulation of intestinal mucin MUC2 expression by short-chain fatty acids: implications for epithelial protection. Biochem J. 2009;420:211-219.

55. Tytgat KM, Opdam FJ, Einerhand AW, et al. MUC2 is the prominent colonic mucin expressed in ulcerative colitis. Gut. 1996;38:554-563.

56. Buisine MP, Desreumaux P, Leteurtre $E$, et al. Mucin gene expression in intestinal epithelial cells in Crohn's disease. Gut. 2001;49:544-551.

57. Willemsen LE, Koetsier MA, van Deventer SJ, et al. Short chain fatty acids stimulate epithelial mucin 2 expression through differential effects on prostaglandin E1 and E2 production by intestinal myofibroblasts. Gut. 2003;52:1442-1447. 
58. Augenlicht L, Shi L, Mariadason J, et al. Repression of MUC2 gene expression by butyrate, a physiological regulator of intestinal cell maturation. Oncogene. 2003;22:4983-4992.

59. Hatayama H, Iwashita J, Kuwajima A, et al. The short chain fatty acid, butyrate, stimulates MUC2 mucin production in the human colon cancer cell line, LS174T. Biochem Biophys Res Commun. 2007;356:599-603.

60. Finnie IA, Dwarakanath AD, Taylor BA, et al. Colonic mucin synthesis is increased by sodium butyrate. Gut. 1995;36:93-99.

61. Gaudier E, Rival M, Buisine MP, et al. Butyrate enemas upregulate Muc genes expression but decrease adherent mucus thickness in mice colon. Physiol Res. 2009;58:111-119.

62. Hoebler C, Gaudier E, De Coppet P, et al. MUC genes are differently expressed during onset and maintenance of inflammation in dextran sodium sulfate-treated mice. Dig Dis Sci. 2006;51:381-389.

63. Shimazu T, Hirschey MD, Newman J, et al. Suppression of oxidative stress by beta-hydroxybutyrate, an endogenous histone deacetylase inhibitor. Science. 2013;339:211-214.

64. Rezaie A, Parker RD, Abdollahi M. Oxidative stress and pathogenesis of inflammatory bowel disease: an epiphenomenon or the cause? Dig Dis Sci. 2007;52:2015-2021.

65. Leufkens AM, van Duijnhoven FJ, Woudt SH, et al. Biomarkers of oxidative stress and risk of developing colorectal cancer: a cohort-nested case-control study in the European Prospective Investigation Into Cancer and Nutrition. Am J Epidemiol. 2012;175:653-663.

66. Russo I, Luciani A, De Cicco P, et al. Butyrate attenuates lipopolysaccharide-induced inflammation in intestinal cells and Crohn's mucosa through modulation of antioxidant defense machinery. PLoS One. 2012;7:e32841. doi:10.1371/journal.pone.0032841.

67. Hamer HM, Jonkers DM, Vanhoutvin SA, et al. Effect of butyrate enemas on inflammation and antioxidant status in the colonic mucosa of patients with ulcerative colitis in remission. Clin Nutr. 2010;29:738-744.

68. Zhu H, Li YR. Oxidative stress and redox signaling mechanisms of inflammatory bowel disease: updated experimental and clinical evidence. Exp Biol Med (Maywood). 2012;237:474-480.

69. Abrahamse SL, Pool-Zobel BL, Rechkemmer G. Potential of short chain fatty acids to modulate the induction of DNA damage and changes in the intracellular calcium concentration by oxidative stress in isolated rat distal colon cells. Carcinogenesis. 1999;20:629-634.

70. Rosignoli P, Fabiani R, De Bartolomeo A, et al. Protective activity of butyrate on hydrogen peroxide-induced DNA damage in isolated human colonocytes and HT29 tumour cells. Carcinogenesis. 2001;22:1675-1680.

71. Wang D, Dubois RN. Eicosanoids and cancer. Nat Rev Cancer. 2010;10:181-193.

72. Sano H, Kawahito Y, Wilder RL, et al. Expression of cyclooxygenase-1 and -2 in human colorectal cancer. Cancer Res. 1995;55:3785-3789.

73. Jahns F, Wilhelm A, Jablonowski N, et al. Butyrate suppresses mRNA increase of osteopontin and cyclooxygenase-2 in human colon tumor tissue. Carcinogenesis. 2011;32:913-920.

74. Tong X, Yin L, Giardina C. Butyrate suppresses Cox-2 activation in colon cancer cells through HDAC inhibition. Biochem Biophys Res Commun. 2004;317:463-471.

75. Crew TE, Elder DJ, Paraskeva C. A cyclooxygenase-2 (COX-2) selective non-steroidal anti-inflammatory drug enhances the growth inhibitory effect of butyrate in colorectal carcinoma cells expressing COX-2 protein: regulation of COX-2 by butyrate. Carcinogenesis. 2000;21:69-77.

76. Karhausen J, Furuta GT, Tomaszewski JE, et al. Epithelial hypoxia-inducible factor-1 is protective in murine experimental colitis. J Clin Invest. 2004;114:1098-1106. 
77. Kelly CJ, Zheng L, Campbell EL, et al. Crosstalk between microbiota-derived short-chain fatty acids and intestinal epithelial HIF augments tissue barrier function. Cell Host Microbe. 2015;17:662-671.

78. Candido EP, Reeves R, Davie JR. Sodium butyrate inhibits histone deacetylation in cultured cells. Cell. 1978;14:105-113.

79. Macia L, Thorburn AN, Binge LC, et al. Microbial influences on epithelial integrity and immune function as a basis for inflammatory diseases. Immunol Rev. 2012;245:164-176.

80. Zimmerman MA, Singh N, Martin PM, et al. Butyrate suppresses colonic inflammation through HDAC1-dependent Fas upregulation and Fas-mediated apoptosis of T cells. Am J Physiol Gastrointest Liver Physiol. 2012;302:G1405-G1415.

81. Chang PV, Hao L, Offermanns S, et al. The microbial metabolite butyrate regulates intestinal macrophage function via histone deacetylase inhibition. Proc Natl Acad Sci USA. 2014;111:2247-2252.

82. Saemann MD, Bohmig GA, Osterreicher $\mathrm{CH}$, et al. Anti-inflammatory effects of sodium butyrate on human monocytes: potent inhibition of IL-12 and up-regulation of IL-10 production. FASEB J. 2000;14:2380-2382.

83. Tedelind S, Westberg F, Kjerrulf M, et al. Anti-inflammatory properties of the short-chain fatty acids acetate and propionate: a study with relevance to inflammatory bowel disease. World J Gastroenterol. 2007;13:2826-2832.

84. Furusawa $\mathrm{Y}$, Obata $\mathrm{Y}$, Fukuda $\mathrm{S}$, et al. Commensal microbe-derived butyrate induces the differentiation of colonic regulatory T cells. Nature. 2013;504:446-450.

85. Arpaia N, Campbell C, Fan X, et al. Metabolites produced by commensal bacteria promote peripheral regulatory T-cell generation. Nature. 2013;504:451-455.

86. Kim M, Qie Y, ParkJ, et al. Gut microbial metabolites fuel host antibody responses. Cell Host Microbe. 2016;20:202-214.

87 Segain JP, Raingeard de la Blétière D, Bourreille A, et al. Butyrate inhibits inflammatory responses through NFkB inhibition: implications for Crohn's disease. Gut. 2000;47:397-403.

88. Vinolo MA, Rodrigues HG, Nachbar RT, et al. Regulation of inflammation by short chain fatty acids. Nutrients. 2011;3:858-876.

89. Maslowski KM, Vieira AT, Ng A, et al. Regulation of inflammatory responses by gut microbiota and chemoattractant receptor GPR43. Nature. 2009;461:1282-1286.

90. Cox MA, Jackson J, Stanton M, et al. Short-chain fatty acids act as antiinflammatory mediators by regulating prostaglandin E2 and cytokines. World J Gastroenterol. 2009;15:5549-5557.

91. Smith PM, Howitt MR, Panikov N, et al. The microbial metabolites, short-chain fatty acids, regulate colonic Treg cell homeostasis. Science. 2013;341:569-573.

92. Hinnebusch BF, Meng S, Wu JT, et al. The effects of short-chain fatty acids on human colon cancer cell phenotype are associated with histone hyperacetylation. J Nutr. 2002;132:10121017.

93. Daly K, Shirazi-Beechey SP. Microarray analysis of butyrate regulated genes in colonic epithelial cells. DNA Cell Biol. 2006;25:49-62.

94. Emenaker NJ, Calaf GM, Cox D, et al. Short-chain fatty acids inhibit invasive human colon cancer by modulating UPA, TIMP-1, TIMP-2, mutant p53, BCl-2, Bax, p21 and PCNA protein expression in an in vitro cell culture model. J Nutr. 2001;131(11 suppl):3041S-3046S.

95. Thangaraju M, Cresci G, Itagaki S, et al. Sodium-coupled transport of the short chain fatty acid butyrate by SLC5A8 and its relevance to colon cancer. J Gastrointest Surg. 2008;12:17731781; discussion 1781-1772. 
96. Vecchia MG, Carnelos Filho M, Fellipe CR, et al. Acetate and propionate potentiate the antiproliferative effect of butyrate on RBL-2H3 growth. Gen Pharmacol. 1997;29:725-728.

97. Fu H, Shi YQ, Mo SJ. Effect of short-chain fatty acids on the proliferation and differentiation of the human colonic adenocarcinoma cell line Caco-2. Chin J Dig Dis. 2004;5:115-117.

98. Butt AJ, Hague A, Paraskeva C. Butyrate- but not TGFbeta1-induced apoptosis of colorectal adenoma cells is associated with increased expression of the differentiation markers E-cadherin and alkaline phosphatase. Cell Death Differ. 1997;4:725-732.

99. Barshishat M, Polak-Charcon S, Schwartz B. Butyrate regulates E-cadherin transcription, isoform expression and intracellular position in colon cancer cells. Br J Cancer. 2000;82:195203.

100. Zuo L, Lu M, Zhou Q, et al. Butyrate suppresses proliferation and migration of RKO colon cancer cells though regulating endocan expression by MAPK signaling pathway. Food Chem Toxicol. 2013;62:892-900.

101. Krishnan M, Singh AB, Smith J, et al. HDAC inhibitors regulate claudin-1 expression in colon cancer cells through modulation of mRNA stability. Oncogene. 2010;29:305-312.

102. Tang $Y$, Chen $Y$, Jiang $H$, et al. G-protein-coupled receptor for short-chain fatty acids suppresses colon cancer. Int J Cancer. 2011;128:847-856.

103. Bordonaro M, Lazarova DL, Sartorelli AC. The activation of beta-catenin by Wnt signaling mediates the effects of histone deacetylase inhibitors. Exp Cell Res. 2007;313:1652-1666.

104. Lazarova DL, Bordonaro M, Carbone R, et al. Linear relationship between Wnt activity levels and apoptosis in colorectal carcinoma cells exposed to butyrate. Int J Cancer. 2004;110:523531.

105. Gibson PR, Moeller I, Kagelari O, et al. Contrasting effects of butyrate on the expression of phenotypic markers of differentiation in neoplastic and non-neoplastic colonic epithelial cells in vitro. J Gastroenterol Hepatol. 1992;7:165-172.

106. Donohoe DR, Collins LB, Wali A, et al. The Warburg effect dictates the mechanism of butyrate-mediated histone acetylation and cell proliferation. Mol Cell. 2012;48:612-626.

107. Singh B, Halestrap AP, Paraskeva C. Butyrate can act as a stimulator of growth or inducer of apoptosis in human colonic epithelial cell lines depending on the presence of alternative energy sources. Carcinogenesis. 1997;18:1265-1270.

108. Kaiko GE, Ryu SH, Koues OI, et al. The colonic crypt protects stem cells from microbiota-derived metabolites. Cell. 2016;165:1708-1720.

109. Malago JJ, Sangu CL. Intraperitoneal administration of butyrate prevents the severity of acetic acid colitis in rats. J Zhejiang Univ Sci B. 2015;16:224-234.

110. Moeinian M, Ghasemi-Niri SF, Mozaffari S, et al. Beneficial effect of butyrate, Lactobacillus casei and L-carnitine combination in preference to each in experimental colitis. World J Gastroenterol. 2014;20:10876-10885.

111. Ferreira TM, Leonel AJ, Melo MA, et al. Oral supplementation of butyrate reduces mucositis and intestinal permeability associated with 5-fluorouracil administration. Lipids. 2012;47:669-678.

112. Song M, Xia B, Li J. Effects of topical treatment of sodium butyrate and 5-aminosalicylic acid on expression of trefoil factor 3 , interleukin 1 beta, and nuclear factor kappaB in trinitrobenzene sulphonic acid induced colitis in rats. Postgrad Med J. 2006;82:130-135.

113. Venkatraman A, Ramakrishna BS, Shaji RV, et al. Amelioration of dextran sulfate colitis by butyrate: role of heat shock protein 70 and NF-kappaB. Am J Physiol Gastrointest Liver Physiol. 2003;285:G177-G184. 
114. Karaki S, Mitsui R, Hayashi H, et al. Short-chain fatty acid receptor, GPR43, is expressed by enteroendocrine cells and mucosal mast cells in rat intestine. Cell Tissue Res. 2006;324:353360.

115. Masui R, Sasaki M, Funaki Y, et al. G protein-coupled receptor 43 moderates gut inflammation through cytokine regulation from mononuclear cells. Inflamm Bowel Dis. 2013;19:28482856.

116. Pacheco RG, Esposito CC, Muller LC, et al. Use of butyrate or glutamine in enema solution reduces inflammation and fibrosis in experimental diversion colitis. World J Gastroenterol. 2012;18:4278-4287.

117. Oliveira AJ, Pinto Júnior FE, Formiga MC, et al. Comparison of prophylactic and therapeutic use of short-chain fatty acid enemas in diversion colitis: a study in Wistar rats. Clinics (Sao Paulo). 2010;65:1351-1356.

118. Vieira EL, Leonel AJ, Sad AP, et al. Oral administration of sodium butyrate attenuates inflammation and mucosal lesion in experimental acute ulcerative colitis. J Nutr Biochem. 2012;23:430-436.

119. Hung TV, Suzuki T. Dietary fermentable fiber reduces intestinal barrier defects and inflammation in colitic mice. J Nutr. 2016;146:1970-1979.

120. Panasevich MR, Allen JM, Wallig MA, et al. Moderately fermentable potato fiber attenuates signs and inflammation associated with experimental colitis in mice. J Nutr. 2015;145:27812788 .

121. Komiyama $Y$, Andoh A, Fujiwara D, et al. New prebiotics from rice bran ameliorate inflammation in murine colitis models through the modulation of intestinal homeostasis and the mucosal immune system. Scand J Gastroenterol. 2011;46:40-52.

122. Arribas B, Suarez-Pereira E, Mellet CO, et al. Di-D-fructose dianhydride-enriched caramels: effect on colon microbiota, inflammation, and tissue damage in trinitrobenzenesulfonic acid-induced colitic rats. J Agr Food Chem. 2010;58:6476-6484.

123. Wang $H$, Shi $P$, Zuo $L$, et al. Dietary non-digestible polysaccharides ameliorate intestinal epithelial barrier dysfunction in IL-10 knockout mice. J Crohns Colitis. 2016;10:1076-1086.

124. Takagi T, Naito Y, Higashimura Y, et al. Partially hydrolysed guar gum ameliorates murine intestinal inflammation in association with modulating luminal microbiota and SCFA. $\mathrm{Br}$ J Nutr. 2016;116:1199-1205.

125. Koleva PT, Valcheva RS, Sun X, et al. Inulin and fructo-oligosaccharides have divergent effects on colitis and commensal microbiota in HLA-B27 transgenic rats. Br J Nutr. 2012;108:16331643.

126. D'Argenio G, Cosenza V, Delle Cave M, et al. Butyrate enemas in experimental colitis and protection against large bowel cancer in a rat model. Gastroenterology. 1996;110:1727-1734.

127. Wong CS, Sengupta S, Tjandra JJ, et al. The influence of specific luminal factors on the colonic epithelium: high-dose butyrate and physical changes suppress early carcinogenic events in rats. Dis Colon Rectum. 2005;48:549-559.

128. Medina $\mathrm{V}$, Afonso JJ, Alvarez-Arguelles $\mathrm{H}$, et al. Sodium butyrate inhibits carcinoma development in a 1,2-dimethylhydrazine-induced rat colon cancer. JPEN J Parenter Enteral Nutr. 1998;22:14-17.

129. Freeman HJ. Effects of differing concentrations of sodium butyrate on 1,2-dimethylhydrazine-induced rat intestinal neoplasia. Gastroenterology. 1986;91:596-602.

130. Caderni G, Luceri C, De Filippo C, et al. Slow-release pellets of sodium butyrate do not modify azoxymethane (AOM)-induced intestinal carcinogenesis in F344 rats. Carcinogenesis. 2001;22:525-527. 
131. Silva FG, Penido LC, Valente FX, et al. Sodium butyrate does not decrease the evolution of precancerous lesions in rats. Acta Cir Bras. 2010;25:507-512.

132. Gaschott T, Steinhilber D, Milovic V, et al. Tributyrin, a stable and rapidly absorbed prodrug of butyric acid, enhances antiproliferative effects of dihydroxycholecalciferol in human colon cancer cells. J Nutr. 2001;131:1839-1843.

133. Heidor R, Furtado KS, Ortega JF, et al. The chemopreventive activity of the histone deacetylase inhibitor tributyrin in colon carcinogenesis involves the induction of apoptosis and reduction of DNA damage. Toxicol Appl Pharmacol. 2014;276:129-135.

134. Deschner EE, Ruperto JF, Lupton JR, et al. Dietary butyrate (tributyrin) does not enhance AOM-induced colon tumorigenesis. Cancer Lett. 1990;52:79-82.

135. Clarke JM, Young GP, Topping DL, et al. Butyrate delivered by butyrylated starch increases distal colonic epithelial apoptosis in carcinogen-treated rats. Carcinogenesis. 2012;33:197202.

136. Sivaprakasam S, Gurav A, Paschall AV, et al. An essential role of Ffar2 (Gpr43) in dietary fibre-mediated promotion of healthy composition of gut microbiota and suppression of intestinal carcinogenesis. Oncogenesis. 2016;5:e238. doi:10.1038/oncsis.2016.38.

137. Singh N, Gurav A, Sivaprakasam S, et al. Activation of Gpr109a, receptor for niacin and the commensal metabolite butyrate, suppresses colonic inflammation and carcinogenesis. Immunity. 2014;40:128-139.

138. Toden S, Lockett TJ, Topping DL, et al. Butyrylated starch affects colorectal cancer markers beneficially and dose-dependently in genotoxin-treated rats. Cancer Biol Ther. 2014;15:1515-1523.

139. Donohoe DR, Holley D, Collins LB, et al. A gnotobiotic mouse model demonstrates that dietary fiber protects against colorectal tumorigenesis in a microbiota- and butyrate-dependent manner. Cancer Discov. 2014;4:1387-1397.

140. Van der Sluis M, De Koning BA, De Bruijn AC, et al. Muc2-deficient mice spontaneously develop colitis, indicating that MUC2 is critical for colonic protection. Gastroenterology. 2006;131:117-129.

141. Rolandelli RH, Koruda MJ, Settle RG, et al. Effects of intraluminal infusion of short-chain fatty acids on the healing of colonic anastomosis in the rat. Surgery. 1986;100:198-204.

142. Bloemen JG, Schreinemacher MH, de Bruine AP, et al. Butyrate enemas improve intestinal anastomotic strength in a rat model. Dis Colon Rectum. 2010;53:1069-1075.

143. Mathew AJ, Wann VC, Abraham DT, et al. The effect of butyrate on the healing of colonic anastomoses in rats. J Invest Surg. 2010;23:101-104.

144. Aguilar-Nascimento JE, Mathie RT, Man WK, et al. Enhanced intra-anastomotic healing by operative lavage with nutrient solutions in experimental left-sided colonic obstruction. Br J Surg. 1995;82:461-464.

145. Topcu O, Karaday K, Kuzu MA, et al. Enteral and intraluminal short-chain fatty acids improves ischemic left colonic anastomotic healing in the rat. Int J Colorectal Dis. 2002;17:171-176.

146. Aguilar-Nascimento JE, Salomao AB, Nochi RJ Jr, et al. Intraluminal injection of short chain fatty acids diminishes intestinal mucosa injury in experimental ischemia-reperfusion. Acta Cir Bras. 2006;21:21-25.

147. Fa-Si-Oen $\mathrm{P}$, van de Gender P, Putter $\mathrm{H}$, et al. The effect of polyethylene glycol and butyrate on anastomotic healing in the rat colon. Tech Coloproctol. 2006;10:308-311.

148. Koruda MJ, Rolandelli RH, Bliss DZ, et al. Parenteral nutrition supplemented with short-chain fatty acids: effect on the small-bowel mucosa in normal rats. Am J Clin Nutr. 1990;51:685-689. 
149. Koruda MJ, Rolandelli RH, Settle RG, et al. Effect of parenteral nutrition supplemented with short-chain fatty acids on adaptation to massive small bowel resection. Gastroenterology. 1988;95:715-720.

150. Rolandelli RH, Buckmire MA, Bernstein KA. Intravenous butyrate and healing of colonic anastomoses in the rat. Dis Colon Rectum. 1997;40:67-70.

151. De Filippo C, Cavalieri D, Di Paola M, et al. Impact of diet in shaping gut microbiota revealed by a comparative study in children from Europe and rural Africa. Proc Natl Acad Sci USA. 2010;107:14691-14696.

152. Reijnders $D$, Goossens $G H$, Hermes GD, et al. Effects of gut microbiota manipulation by antibiotics on host metabolism in obese humans: a randomized double-blind placebo-controlled trial. Cell Metabol. 2016;24:63-74.

153. Huda-Faujan N, Abdulamir AS, Fatimah AB, et al. The impact of the level of the intestinal short chain fatty acids in inflammatory bowel disease patients versus healthy subjects. Open Biochem J. 2010;4:53-58.

154. Kumari R, Ahuja $\mathrm{V}$, Paul J. Fluctuations in butyrate-producing bacteria in ulcerative colitis patients of North India. World J Gastroenterol. 2013;19:3404-3414.

155. Joossens M, Huys G, Cnockaert M, et al. Dysbiosis of the faecal microbiota in patients with Crohn's disease and their unaffected relatives. Gut. 2011;60:631-637.

156. Machiels K, Joossens M, Sabino J, et al. A decrease of the butyrate-producing species Roseburia hominis and Faecalibacterium prausnitzii defines dysbiosis in patients with ulcerative colitis. Gut. 2014;63:1275-1283.

157. Galecka M, Szachta P, Bartnicka A, et al. Faecalibacterium prausnitzii and Crohn's disease - is there any connection? Pol J Microbiol. 2013;62:91-95.

158. Renom G, Bulois P, Hafraoui S, et al. Simple gas chromatography analysis of faecal butyrate: application to patients at risk of pouchitis. Clin Chem Lab Med. 2001;39:15-19.

159. Sagar PM, Taylor BA, Godwin P, et al. Acute pouchitis and deficiencies of fuel. Dis Colon Rectum. 1995;38:488-493.

160. Clausen MR, Tvede M, Mortensen PB. Short-chain fatty acids in pouch contents from patients with and without pouchitis after ileal pouch-anal anastomosis. Gastroenterology. 1992;103:1144-1153.

161. Hove H, Mortensen PB. Short-chain fatty acids in the non-adapted and adapted pelvic ileal pouch. Scand J Gastroenterol. 1996;31:568-574.

162. Wischmeyer P, Pemberton JH, Phillips SF. Chronic pouchitis after ileal pouch-anal anastomosis: responses to butyrate and glutamine suppositories in a pilot study. Mayo Clin Proc. 1993;68:978-981.

163. Morgan XC, Tickle TL, Sokol $\mathrm{H}$, et al. Dysfunction of the intestinal microbiome in inflammatory bowel disease and treatment. Genome Biol. 2012;13:R79. doi:10.1186/gb-2012-13-9-r79.

164. De Preter $V$, Bulteel $V$, Suenaert $P$, et al. Pouchitis, similar to active ulcerative colitis, is associated with impaired butyrate oxidation by intestinal mucosa. Inflamm Bowel Dis. 2009;15:335-340.

165. Finnie IA, Taylor BA, Rhodes JM. Ileal and colonic epithelial metabolism in quiescent ulcerative colitis: increased glutamine metabolism in distal colon but no defect in butyrate metabolism. Gut. 1993;34:1552-1558.

166. Boesmans L, Ramakers M, Arijs I, et al. Inflammation-induced downregulation of butyrate uptake and oxidation is not caused by a reduced gene expression. J Cell Physiol. 2015;230:418-426. 
167. Vernia P, Caprilli R, Latella G, et al. Fecal lactate and ulcerative colitis. Gastroenterology. 1988;95:1564-1568.

168. Vertzoni M, Goumas K, Soderlind E, et al. Characterization of the ascending colon fluids in ulcerative colitis. Pharm Res. 2010;27:1620-1626.

169. Lambert DW, Wood IS, Ellis A, et al. Molecular changes in the expression of human colonic nutrient transporters during the transition from normality to malignancy. Br J Cancer. 2002;86:1262-1269.

170. Li H, Myeroff L, Smiraglia D, et al. SLC5A8, a sodium transporter, is a tumor suppressor gene silenced by methylation in human colon aberrant crypt foci and cancers. Proc Natl Acad Sci USA. 2003;100:8412-8417.

171. Brown DG, Rao S, Weir TL, et al. Metabolomics and metabolic pathway networks from human colorectal cancers, adjacent mucosa, and stool. Cancer Metab. 2016;4:11. doi:10.1186/ s40170-016-0151-y.

172. Burkitt DP. Epidemiology of cancer of the colon and rectum. Cancer. 1971;28:3-13.

173. O'Keefe SJ, Chung D, Mahmoud N, et al. Why do African Americans get more colon cancer than Native Africans? J Nutr. 2007;137(1 suppl):175S-182S.

174. Ou J, Carbonero F, Zoetendal EG, et al. Diet, microbiota, and microbial metabolites in colon cancer risk in rural Africans and African Americans. Am J Clin Nutr. 2013;98:111-120.

175. Ou J, DeLany JP, Zhang M, et al. Association between low colonic short-chain fatty acids and high bile acids in high colon cancer risk populations. Nutr Cancer. 2012;64:34-40.

176. Center MM, Jemal A, Ward E. International trends in colorectal cancer incidence rates. Cancer Epidemiol Biomarkers Prev. 2009;18:1688-1694.

177. Clausen MR, Bonnen H, Mortensen PB. Colonic fermentation of dietary fibre to short chain fatty acids in patients with adenomatous polyps and colonic cancer. Gut. 1991;32:923-928.

178. Weaver GA, Krause JA, Miller TL, et al. Short chain fatty acid distributions of enema samples from a sigmoidoscopy population: an association of high acetate and low butyrate ratios with adenomatous polyps and colon cancer. Gut. 1988;29:1539-1543.

179. Weir TL, Manter DK, Sheflin AM, et al. Stool microbiome and metabolome differences between colorectal cancer patients and healthy adults. PLoS One. 2013;8:e70803. doi:10.1371/journal.pone.0070803.

180. Bradburn DM, Mathers JC, Gunn A, et al. Colonic fermentation of complex carbohydrates in patients with familial adenomatous polyposis. Gut. 1993;34:630-636.

181. Breuer RI, Buto SK, Christ ML, et al. Rectal irrigation with short-chain fatty acids for distal ulcerative colitis. Preliminary report. Dig Dis Sci. 1991;36:185-187.

182. Steinhart AH, Brzezinski A, Baker JP. Treatment of refractory ulcerative proctosigmoiditis with butyrate enemas. Am J Gastroenterol. 1994;89:179-183.

183. Patz J, Jacobsohn WZ, Gottschalk-Sabag S, et al. Treatment of refractory distal ulcerative colitis with short chain fatty acid enemas. Am J Gastroenterol. 1996;91:731-734.

184. Vernia P, Cittadini M, Caprilli R, et al. Topical treatment of refractory distal ulcerative colitis with 5-ASA and sodium butyrate. Dig Dis Sci. 1995;40:305-307.

185. Scheppach W, Sommer H, Kirchner T, et al. Effect of butyrate enemas on the colonic mucosa in distal ulcerative colitis. Gastroenterology. 1992;103:51-56.

186. Senagore AJ, MacKeigan JM, Scheider M, et al. Short-chain fatty acid enemas: a cost-effective alternative in the treatment of nonspecific proctosigmoiditis. Dis Colon Rectum. 1992;35:923-927. 
187. Steinhart AH, Hiruki T, Brzezinski A, et al. Treatment of left-sided ulcerative colitis with butyrate enemas: a controlled trial. Aliment Pharmacol Ther. 1996;10:729-736.

188. Scheppach W. Treatment of distal ulcerative colitis with short-chain fatty acid enemas. A placebo-controlled trial. German-Austrian SCFA Study Group. Dig Dis Sci. 1996;41:2254-2259.

189. Scheppach W, Muller JG, Boxberger F, et al. Histological changes in the colonic mucosa following irrigation with short-chain fatty acids. Eur J Gastroenterol Hepatol. 1997;9:163-168.

190. Breuer RI, Soergel KH, Lashner BA, et al. Short chain fatty acid rectal irrigation for left-sided ulcerative colitis: a randomised, placebo controlled trial. Gut. 1997;40:485-491.

191. Lührs H, Gerke T, Muller JG, et al. Butyrate inhibits NF-kappaB activation in lamina propria macrophages of patients with ulcerative colitis. Scand J Gastroenterol. 2002;37:458-466.

192. Vernia P, Annese V, Bresci G, et al. Topical butyrate improves efficacy of 5-ASA in refractory distal ulcerative colitis: results of a multicentre trial. Eur J Clin Invest. 2003;33:244-248.

193. Vernia P, Marcheggiano A, Caprilli R, et al. Short-chain fatty acid topical treatment in distal ulcerative colitis. Aliment Pharmacol Ther. 1995;9:309-313.

194. Vernia P, Monteleone G, Grandinetti G, et al. Combined oral sodium butyrate and mesalazine treatment compared to oral mesalazine alone in ulcerative colitis: randomized, double-blind, placebo-controlled pilot study. Dig Dis Sci. 2000;45:976-981.

195. Galvez J, Rodriguez-Cabezas ME, Zarzuelo A. Effects of dietary fiber on inflammatory bowel disease. Mol Nutr Food Res. 2005;49:601-608.

196. Fernandez-Banares F, Hinojosa J, Sanchez-Lombrana JL, et al. Randomized clinical trial of Plantago ovata seeds (dietary fiber) as compared with mesalamine in maintaining remission in ulcerative colitis. Am J Gastroenterol. 1999;94:427-433.

197. Martinez CA, de Campos FG, de Carvalho VR, et al. Claudin-3 and occludin tissue content in the glands of colonic mucosa with and without a fecal stream. J Mol Histol. 2015;46:183-194.

198. Korelitz BI, Cheskin LJ, Sohn N, et al. Proctitis after fecal diversion in Crohn's disease and its elimination with reanastomosis: implications for surgical management. Report of four cases. Gastroenterology. 1984;87:710-713.

199. Harig JM, Soergel KH, Komorowski RA, et al. Treatment of diversion colitis with short-chainfatty acid irrigation. N Engl J Med. 1989;320:23-28.

200. Kiely EM, Ajayi NA, Wheeler RA, et al. Diversion procto-colitis: response to treatment with short-chain fatty acids. J Pediatr Surg. 2001;36:1514-1517.

201. Pal K, Tinalal S, Al Buainain $\mathrm{H}$, et al. Diversion proctocolitis and response to treatment with short-chain fatty acids - a clinicopathological study in children. Indian J Gastroenterol. 2015;34:292-299.

202. Komorowski RA. Histologic spectrum of diversion colitis. Am J Surg Pathol. 1990;14:548-554.

203. Guillemot F, Colombel JF, Neut C, et al. Treatment of diversion colitis by short-chain fatty acids. Prospective and double-blind study. Dis Colon Rectum. 1991;34:861-864.

204. Schauber J, Bark T, Jaramillo E, et al. Local short-chain fatty acids supplementation without beneficial effect on inflammation in excluded rectum. Scand J Gastroenterol. 2000;35:184189.

205. Luceri C, Femia AP, Fazi M, et al. Effect of butyrate enemas on gene expression profiles and endoscopic/histopathological scores of diverted colorectal mucosa: a randomized trial. Dig Liver Dis. 2016;48:27-33.

206. Mohammed N, Kestin L, Ghilezan M, et al. Comparison of acute and late toxicities for three modern high-dose radiation treatment techniques for localized prostate cancer. Int J Radiation Oncol Biol Physics. 2012;82:204-212. 
207. Mamel JJ, Chen M, Combs W, et al. Short-chain fatty acids (SCFA) enemas are useful for the treatment of chronic radiation proctitis. Gastroenterology. 1995;108(suppl 1):A305. doi:10.1016/0016-5085(95)23880-8.

208. Al-Sabbagh R, Sinicrope FA, Sellin JH, et al. Evaluation of short-chain fatty acid enemas: treatment of radiation proctitis. Am J Gastroenterol. 1996;91:1814-1816.

209. Talley NA, Chen F, King D, et al. Short-chain fatty acids in the treatment of radiation proctitis: a randomized, double-blind, placebo-controlled, cross-over pilot trial. Dis Colon Rectum. 1997;40:1046-1050.

210. Pinto A, Fidalgo P, Cravo M, et al. Short chain fatty acids are effective in short-term treatment of chronic radiation proctitis: randomized, double-blind, controlled trial. Dis Colon Rectum. 1999;42:788-795; discussion 795-786.

211. Vernia P, Fracasso PL, Casale V, et al. Topical butyrate for acute radiation proctitis: randomised, crossover trial. Lancet. 2000;356:1232-1235.

212. Hille A, Herrmann MK, Kertesz T, et al. Sodium butyrate enemas in the treatment of acute radiation-induced proctitis in patients with prostate cancer and the impact on late proctitis. A prospective evaluation. Strahlenther Onkol. 2008;184:686-692.

213. Maggio A, Magli A, Rancati T, et al. Daily sodium butyrate enema for the prevention of radiation proctitis in prostate cancer patients undergoing radical radiation therapy: results of a multicenter randomized placebo-controlled dose-finding phase 2 study. Int J Radiat Oncol Biol Phys. 2014;89:518-524.

214. Shadad AK, Sullivan FJ, Martin JD, et al. Gastrointestinal radiation injury: symptoms, risk factors and mechanisms. World J Gastroenterol. 2013;19:185-198.

215. Tonelli F, Dolara P, Batignani G, et al. Effects of short chain fatty acids on mucosal proliferation and inflammation of ileal pouches in patients with ulcerative colitis and familial polyposis. Dis Colon Rectum. 1995;38:974-978.

216. Welters CF, Heineman E, Thunnissen FB, et al. Effect of dietary inulin supplementation on inflammation of pouch mucosa in patients with an ileal pouch-anal anastomosis. Dis Colon Rectum. 2002;45:621-627.

217. Di Sabatino A, Morera R, Ciccocioppo R, et al. Oral butyrate for mildly to moderately active Crohn's disease. Aliment Pharmacol Ther. 2005;22:789-794.

218. Le Leu RK, Winter JM, Christophersen CT, et al. Butyrylated starch intake can prevent red meat-induced 06-methyl-2-deoxyguanosine adducts in human rectal tissue: a randomised clinical trial. Brit J Nutr. 2015;114:220-230.

219. van Munster IP, Tangerman A, Nagengast FM. Effect of resistant starch on colonic fermentation, bile acid metabolism, and mucosal proliferation. Dig Dis Sci. 1994;39:834-842.

220. Heijnen ML, van Amelsvoort JM, Deurenberg P, et al. Limited effect of consumption of uncooked (RS2) or retrograded (RS3) resistant starch on putative risk factors for colon cancer in healthy men. Am J Clin Nutr. 1998;67:322-331.

221. Dronamraju SS, Coxhead JM, Kelly SB, et al. Cell kinetics and gene expression changes in colorectal cancer patients given resistant starch: a randomised controlled trial. Gut. 2009;58:413-420.

222. Mathers JC, Movahedi M, Macrae F, et al. Long-term effect of resistant starch on cancer risk in carriers of hereditary colorectal cancer: an analysis from the CAPP2 randomised controlled trial. Lancet Oncol. 2012;13:1242-1249.

223. O'Keefe SJ, Li JV, Lahti L, et al. Fat, fibre and cancer risk in African Americans and rural Africans. Nat Commun. 2015;6:6342. doi:10.1038/ncomms7342. 
224. Park Y, Hunter DJ, Spiegelman D, et al. Dietary fiber intake and risk of colorectal cancer: a pooled analysis of prospective cohort studies. JAMA. 2005;294:2849-2857.

225. Orlich MJ, Singh PN, Sabate J, et al. Vegetarian dietary patterns and the risk of colorectal cancers. JAMA Intern Med. 2015;175:767-776.

226. Triff K, Kim E, Chapkin RS. Chemoprotective epigenetic mechanisms in a colorectal cancer model: Modulation by n-3 PUFA in combination with fermentable fiber. Curr Pharmacol Rep. 2015;1:11-20.

227. Patnaik A, Rowinsky EK, Villalona MA, et al. A phase I study of pivaloyloxymethyl butyrate, a prodrug of the differentiating agent butyric acid, in patients with advanced solid malignancies. Clin Cancer Res. 2002;8:2142-2148.

228. Conley BA, Egorin MJ, Tait N, et al. Phase I study of the orally administered butyrate prodrug, tributyrin, in patients with solid tumors. Clin Cancer Res. 1998;4:629-634.

229. Gore SD, Weng LJ, Figg WD, et al. Impact of prolonged infusions of the putative differentiating agent sodium phenylbutyrate on myelodysplastic syndromes and acute myeloid leukemia. Clin Cancer Res. 2002;8:963-970.

230. Thibault A, Cooper MR, Figg WD, et al. A phase I and pharmacokinetic study of intravenous phenylacetate in patients with cancer. Cancer Res. 1994;54:1690-1694.

231. Thibault A, Samid D, Cooper MR, et al. Phase I study of phenylacetate administered twice daily to patients with cancer. Cancer. 1995;75:2932-2938.

232. Gilbert J, Baker SD, Bowling MK, et al. A phase I dose escalation and bioavailability study of oral sodium phenylbutyrate in patients with refractory solid tumor malignancies. Clin Cancer Res. 2001;7:2292-2300.

233. Reid T, Valone F, Lipera W, et al. Phase II trial of the histone deacetylase inhibitor pivaloyloxymethyl butyrate (Pivanex, AN-9) in advanced non-small cell lung cancer. Lung Cancer. 2004;45:381-386.

234. Ram E, Sherman $Y$, Weil $R$, et al. Is mechanical bowel preparation mandatory for elective colon surgery? A prospective randomized study. Arch Surg. 2005;140:285-288.

235. Miettinen RP, Laitinen ST, Makela JT, et al. Bowel preparation with oral polyethylene glycol electrolyte solution vs. no preparation in elective open colorectal surgery: prospective, randomized study. Dis Colon Rectum. 2000;43:669-675; discussion 675-667.

236. Burke $\mathrm{P}$, Mealy K, Gillen $\mathrm{P}$, et al. Requirement for bowel preparation in colorectal surgery. BrJ Surg. 1994;81:907-910.

237. Russo F, Linsalata M, Clemente C, et al. Inulin-enriched pasta improves intestinal permeability and modifies the circulating levels of zonulin and glucagon-like peptide 2 in healthy young volunteers. Nutr Res. 2012;32:940-946.

238. Hamer HM, Jonkers DM, Renes IB, et al. Butyrate enemas do not affect human colonic MUC2 and TFF3 expression. Eur J Gastroenterol Hepatol. 2010;22:1134-1140.

239. Hamer HM, Jonkers DM, Bast A, et al. Butyrate modulates oxidative stress in the colonic mucosa of healthy humans. Clin Nutr. 2009;28:88-93.

240. Mortensen FV, Langkilde NC, Joergensen JC, et al. Short-chain fatty acids stimulate mucosal cell proliferation in the closed human rectum after Hartmann's procedure. Int J Colorectal Dis. 1999;14:150-154.

241. Wedlake L, Slack N, Andreyev HJ, et al. Fiber in the treatment and maintenance of inflammatory bowel disease: a systematic review of randomized controlled trials. Inflamm Bowel Dis. 2014;20:576-586. 
242. Assisi RF, GISDI Study Group. Combined butyric acid/mesalazine treatment in ulcerative colitis with mild-moderate activity. Results of a multicentre pilot study. Minerva Gastroenterol Dietol. 2008;54:231-238.

243. Leek BT, Tasto JP, Tibor LM, et al. Augmentation of tendon healing with butyric acid-impregnated sutures: biomechanical evaluation in a rabbit model. Am J Sports Med. 2012;40:1762-1771 



$$
8
$$




\section{CHAPTER 8}

General discussion

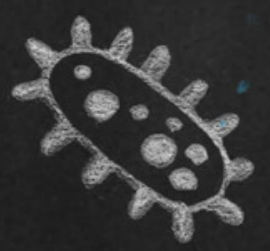



The alarming increase in the prevalence of the metabolic syndrome can be attributed to a change in dietary habits and lack of physical activity. Increasing the amount of fiber ingestion seems a potential strategy both in the treatment and in the prevention of the metabolic syndrome..$^{1-3}$ Our understanding of the underlying mechanisms by which fibers regulate human metabolism is, however, limited. Fermentation of these fibers by the gut microbiota results in the production of the short-chain fatty acids (SCFAs) acetate, propionate, and butyrate. These end products have been suggested to contribute to the beneficial effects of fiber intake on colonic health as well as metabolic health. As shown in Chapter $\mathbf{1}$, in vitro and animal studies suggest that SCFAs influence several metabolic pathways. Human data are, however, scarce. In this thesis, we aimed to address several important issues raised in Chapter 1. More specifically, we determined the effects of colonic SCFA supplementation on human metabolism and aimed to unravel the underlying mechanisms contributing to these effects (Chapter 2 and Chapter 5). In addition, we translated these effects into a more physiological situation, investigating the metabolic effects of short term (Chapter 3) and long term (Chapter 4) prebiotic supplementation in overweight to obese subjects. Last, we investigated the therapeutic options of SCFA application in colonic health (Chapter 6 and Chapter 7).

\section{EFFECTS OF COLONIC SHORT-CHAIN FATTY ACIDS ON HUMAN METABOLISM}

SCFAs produced by microbial fermentation are believed to play a role in human metabolism. In vitro and animal studies have shown that SCFAs are involved in energy regulation, lipid metabolism, glucose homeostasis, and low-grade inflammation. ${ }^{4}$ Several studies indicated that the luminal and circulating most abundantly present SCFA acetate is an important mediator of substrate and energy metabolism. 5,6 However, there is also evidence from a rodent study that a high concentration of acetate offered to the brain would promote the metabolic syndrome rather than improving insulin sensitivity and body weight control. ${ }^{7}$ As a result, in Chapter 2, we investigated the effects of colonic acetate infusions in obese human subjects. We demonstrated that distal colonic acetate infusions pronouncedly increased fasting fat oxidation by more than $25 \%$ and increased fasting plasma PYY concentrations. In addition, distal colonic acetate administration tended to raise fasting circulating acetate, as well as it tended to decrease circulating TNF-a concentrations. These observations suggest that acetate might be an important nutritional target to beneficially influence human metabolism. 
SCFA production occurs predominantly in the cecum and proximal part of the colon. ${ }^{8}$ In other human intervention studies, SCFAs have been administered at several locations, i.e. orally, ${ }^{9-11}$ intragastrically, ${ }^{12}$ intravenously, ${ }^{13-15}$ and rectally, ${ }^{13,16,17}$ but not in the proximal colon. Therefore, we investigated in Chapter 2 if downstream metabolic effects of SCFA administration differ when SCFAs are infused in the proximal compared with the distal colon. We demonstrated that, in contrast with what we found for distal acetate supplementation, proximal acetate administration did not affect human metabolism. We suggest that this difference in effects can be attributed to a different abundance of various SCFA receptors throughout the human intestine. Indeed, a previous study has shown that GPR43 expression increased from the proximal to the distal part of the human colon. ${ }^{18}$ In contrast, animal studies showed that GPR41 expression in mice is highest in the proximal colon compared with the middle or distal colon. ${ }^{19}$ These inconsistent findings led us to investigate in Chapter 5 the expression pattern of the SCFA receptors GPR41, GPR43, and GPR109a, and the satiety hormones PYY and GLP-1, throughout the human ileum and colon. We observed that expression of GPR41, GRP43, and GPR109a, as well as PYY and proglucagon, as a precursor for GLP-1, was highest in the ileum compared with the colon.

The results of our two studies seem contradictory: We only found metabolic effects after distal acetate infusion, while SCFA receptor expression was low in that part of the gut. There are several explanations for this discrepancy. First, we assume that SCFA receptor activation is not the only mechanism by which SCFAs influence human metabolism. Distally administered acetate bypasses the liver and becomes systemically available for metabolically active organs, such as muscle or adipose tissue. Indeed, we showed a trend towards higher plasma acetate concentrations after distal colonic acetate infusion, but not after proximal infusions. Therefore, it is important to know the exact fate of colonic SCFAs in order to establish the contribution of colonic SCFA receptor activation vs specific targeted organs. Boets et al. $^{20}$ have investigated the fate of colonic derived SCFAs in healthy subjects with a mean BMI of $22 \mathrm{~kg} / \mathrm{m}^{2}$ by delivering known amounts of capsulated ${ }^{13} \mathrm{C}$-labeled SCFAs into the proximal colon. They found that the fraction of colonic derived acetate, propionate, and butyrate appearing in plasma was 36\%, 9\%, and 2\% respectively. Also almost $15 \%$ of the infused acetate was used for fatty acid formation, and $6 \%$ of colonic delivered propionate was used for gluconeogenesis in the liver. Moreover, it was confirmed that contact with bacteria is essential for interconversion of the SCFAs, for example incorporation of ${ }^{13} \mathrm{C}$-acetate into butyrate. This unique study provides important mechanistic insight into SCFA uptake and release into the systemic circulation, and supports the hypothesis that systemically available SCFAs have metabolic effects. This specific study used specially coated capsules, designed to dissolve at a pH > 7.0, and Boets and colleagues have confirmed that the capsules released their content into the proximal colon, 380 - 400 min after intake. Up to now, various strategies have been used to deliver compounds specifically into the colon, including coating with $\mathrm{pH}$-sensitive polymers, formulation of timed released 
systems, exploitation of carriers that are degraded specifically by colonic bacteria, bioadhesive systems and osmotic controlled drug delivery systems. ${ }^{21}$ An interesting approach is the use of a carrier which disintegrates after contact with colonic bacteria. Chambers et al.22 made use of a specific characteristic of the prebiotic inulin, and used this dietary fiber as a vector to deliver propionate into the colon. As inulin is an easily fermented prebiotic, it will release the attached propionate in the proximal colon. For future research, it will be interesting to use an approach, by specifically designed capsules or a slowly fermentable prebiotic as vector, to deliver ${ }^{13} \mathrm{C}$-labeled SCFAs into the distal part of the colon, to compare the possible different fates of proximal and distal administered SCFAs. Moreover, as it has been postulated that a different microbial composition between lean and obese volunteers accounts for a different fermentation profile, ${ }^{23}$ it will be even more interesting to execute these investigations in an obese population.

Moreover, we assume that fiber ingestion can stimulate SCFA receptor expression, as was previously shown in rats. ${ }^{18,24}$ As fermentation is highest in the proximal part of the colon due to higher substrate availability, it is possible that the higher fermentation rate at that location results in a higher expression of SCFA receptors. However, it is not known if a higher SCFA availability is the direct cause of GPR stimulation, or that the higher GPR abundance is a direct effect of fibers themselves. Nevertheless, ingesting slow-fermentable fibers, that are fermented in the distal part of the gut, might stimulate expression of SCFA receptors more distally in the gut and increase downstream metabolic signaling. The role of SCFA receptors in metabolism has been investigated before, mainly by studying effects in GPR knockout mice. ${ }^{5,25-29}$ Importantly, a recent study has shown the limitations of using a mouse model to study human physiology regarding SCFA receptors. ${ }^{30}$ This study showed that the immunological response of mouse monocytes to acetate via GPR stimulation is different from human monocytes. This discrepancy between mouse models and the human situation was already reported before. ${ }^{31}$ Therefore, it is important to perform studies in human subjects, or use human material, for future research. An interesting approach will be to investigate colonic tissue of human subjects after ingestion of a slow-fermentable fiber. The slow-fermentable fiber is expected to stimulate GPR expression in the distal part of the colon, a site that can be easily reached for tissue sampling. Moreover, colonic tissue from healthy volunteers should be used in ex vivo studies to confirm the metabolic effects of SCFAs found in in vitro or animal studies. It will be interesting to expose this tissue to several conditions and/or SCFA receptor agonists or antagonists to investigate metabolic effects at the level of the gut. 


\section{EFFECTS OF PREBIOTICS ON METABOLIC HEALTH}

As described in Chapter 1, several human studies have shown that ingestion of prebiotics, and in particular inulin and galacto-oligosaccharides (GOS) have important metabolic effects. Longer-term ingestion of inulin had a positive effect on body weight, BMI, as well as energy and fat intake in patients with type 2 diabetes. ${ }^{32}$ Moreover, inulin and GOS supplementation for several weeks resulted in an improved insulin and lipid homeostasis and attenuated low-grade systemic inflammation in overweight and elderly subjects. ${ }^{33-41}$ In vitro models have shown that inulin and GOS are both fermented into SCFAs in considerable amounts. ${ }^{42-47}$ However, no studies have investigated if prebiotic-derived SCFAs contribute to the effects of prebiotics on human metabolism. As a result, in Chapter 3 we investigated the acute metabolic effects of inulin, and used stable isotope tracer methodology to study if these effects could be attributed to SCFA formation in overweight to obese subjects. We found that the fermentable inulin increased fat oxidation and reduced the postprandial free fatty acid (FFA) response directly after intake compared with the digestible placebo maltodextrin. Moreover, inulin ingestion caused a lower postprandial glucose and insulin response compared with maltodextrin. Importantly, we found that inulin intake increased plasma acetate concentrations and decreased plasma FFAs in the late postprandial period compared with maltodextrin. Tracer methodology revealed that inulin was fermented into all three SCFAs, as indicated by appearance of ${ }^{13} \mathrm{C}$-acetate, ${ }^{13} \mathrm{C}$-butyrate, and ${ }^{13} \mathrm{C}$-propionate in feces and blood. We suggest that the metabolic effects of inulin can be attributed to two specific characteristics of the prebiotic. First, the lower glycemic index of inulin compared with maltodextrin could have promoted the metabolic effects in the early postprandial phase. In comparison to maltodextrin (the placebo used in the trial) inulin intake resulted in a lower rise in postprandial blood glucose concentrations, and therefore also lower insulin response, leading to an attenuated postprandial decline in FFAs and higher fat oxidation. Second, the fermentation of inulin into SCFAs, as indicated by an increase in plasma acetate as well as formation of ${ }^{13} \mathrm{C}$-SCFAs, might explain the metabolic improvements in the late postprandial period, i.e. a decrease in plasma FFAs. Interestingly, a recent study has shown that an inulin-propionate ester significantly reduced weight gain, intra-abdominal adipose tissue distribution, intracellular hepatic lipid content and prevented the deterioration in insulin sensitivity when compared with inulin alone. ${ }^{22}$ Therefore, an interesting future approach will be to link the inulin with a specific SCFA, for instance acetate. Inulin is then used as a vector and when it reaches the microbiota, it will be fermented, thereby releasing the linked acetate. It can be hypothesized that both the fermentation of inulin and the release of the specific SCFA will lead to a high colonic concentration, and thus a higher systemic availability of the linked SCFA. 
To investigate whether the acute metabolic effects investigated in Chapter 3 translate into long-term outcome, we performed a twelve-week supplementation trial with the prebiotic GOS in prediabetic subjects and assessed metabolic effects, as well as effects on gut microbiota, as described in Chapter 4. Remarkably, while a five-fold increase in Bifidobacterium was found compared to placebo, we did not find any effect of GOS supplementation on plasma and fecal SCFA concentrations, hormones, or markers of inflammation. In addition, no significant alterations in peripheral and adipose tissue insulin sensitivity, body composition, and energy and substrate metabolism were found compared with placebo. The fact that GOS is an easily fermentable fiber, meaning that most of the fermentation occurs in the proximal colon, might have attributed to the lack of metabolic response. As shown in Chapter 2, most metabolic effects were found after distal acetate administration, therefore it could be hypothesized that the colonically formed SCFAs are scavenged by the liver before they reach peripheral metabolic organs. Indeed, Boets et $a 1 .{ }^{20}$ have found a splanchnic extraction of 64\% for acetate and $91 \%$ for propionate, meaning that only $36 \%$ of proximally colonic administered acetate and $9 \%$ of proximally colonic administered propionate reached the systemic circulation. It has been shown that SCFAs also beneficially affect liver function, i.e. activate AMPK and increase substrate oxidation. ${ }^{6}$ However, since, Boets et al. ${ }^{20}$ showed that almost $15 \%$ of colonic acetate is used for FFA production, and $6 \%$ of propionate is used for gluconeogenesis in the liver, it is conceivable that the overall metabolic response is lower when a part of the SCFAs is scavenged by the liver compared with the metabolic response when SCFAs are available for peripheral metabolic tissues, such as muscle and adipose tissue. Moreover, it has recently been shown that systemically circulating acetate can be taken up by the brain to suppress appetite. ${ }^{48}$ Therefore, it will be interesting to investigate the metabolic effects of slow-fermentable fibers, which are fermented in the distal part of the gut. In addition, we were not able to take colonic biopsies in this study, which would have been interesting to investigate whether prebiotic supplementation is able to stimulate GPR expression in humans, as is shown for rats. 18,24

As reported in Chapter 4 and by others, ${ }^{49}$ the microbial composition can rapidly change by changing the diet. More importantly, the data presented in Chapter $\mathbf{4}$ are in line with a recent human study, showing that modification of the gut microbiota by ingesting antibiotics did not automatically translate into effects on host metabolism. ${ }^{50}$ Both of these studies are in contrast with rodent studies and highlight the importance of performing human trials to investigate metabolism Moreover, it has been suggested that due to differences in microbial composition between human subjects, there are so-called responders and non-responders to treatment..$^{51}$ A recent study presented evidence for marked inter-individual variability in postprandial glycemic responses to identical meals. This variability was associated with microbial composition and function. ${ }^{52}$ Another interesting study showed that there is a large inter-individual variation in glucose metabolism following a three-day consumption of barley kernel based bread. ${ }^{53}$ The authors divided the study subjects into 
a group which responded the least or had no improvement in glucose homeostasis and a group which responded the best after prebiotic treatment and showed that the microbial composition was changed in the responders, but not in the non-responders. Specifically, the abundance of the genus Prevotella, which belongs to the Bacteroidetes phylum, was increased in the responding group. Importantly, there were no differences in fecal SCFAs between the groups. This approach is novel and reveals that data need to be confirmed in larger, preferably prospective, studies. It may in the future lead to more patient-specific diagnostics and treatments based on microbiota composition. In the study presented in Chapter 4, all subjects showed an increase in bifidobacteria. We could not identify responders and non-responders with regards to the primary outcome insulin sensitivity in our study of 44 subjects. Overall, this is still an emerging field with many challenges and limitations, as several factors are known to influence microbiota composition, such as medication use, ageing, activity level, and even time of the day. ${ }^{54-57}$

\section{THERAPEUTIC OPTIONS OF COLONIC SHORT-CHAIN FATTY ACID ADMINISTRATION}

In contrast to acetate and propionate, which are thought to have mostly metabolic effects on peripheral tissues such as muscle and adipose tissue, butyrate is considered most important for maintenance of colonic health, primarily by acting as a direct energy source for colonocytes. Butyrate is almost completely metabolised by colonocytes and is therefore only found in small amounts in peripheral blood. ${ }^{58,59}$ The use of SCFA, and especially butyrate, enemas seems promising for treating colonic diseases. However high systemic concentrations of butyrate can cause side effects such as hypokalemia and nausea. ${ }^{60,61}$ Therefore, we investigated the inter-organ trafficking of a butyrate enema in Chapter 6 in a surgical setting. We showed an increased release of butyrate from the gut shortly after rectal butyrate administration, combined with an increased butyrate uptake by the liver. As a result, there was no splanchnic release of butyrate in the subjects, suggesting that the liver is capable to metabolize all rectally administered butyrate that is released into the systemic circulation. This is in line with a recent study from Boets et $a l .{ }^{20}$ who showed, using stable isotopes, that the fraction of colonic applied butyrate appearing in plasma is only $2 \%$. Using this approach, Boets et al. ${ }^{20}$ could, however, not determine the amount of SCFAs that is released by the gut after butyrate supplementation. In Chapter 6 we showed that only $18.3 \%$ of the colonic administered butyrate was released from the gut into the portal vein within 30 min after administration. As previous work by our group already established that the liver has a large capacity to capture butyrate, ${ }^{59}$ we concluded that it is safe to use butyrate enemas as treatment for colonic disease. In addition, it will 
be interesting to investigate the colonic release and hepatic clearance of acetate from distal colonic acetate supplementation, as we have shown in Chapter 2 that distal acetate enemas have metabolic effects while proximal enemas do not have metabolic effects.

In Chapter 7, we reviewed the effects of SCFA enemas on colonic diseases in humans. We showed that in vitro and ex vivo studies point towards SCFAs to be important factors to maintain metabolic homeostasis in colonocytes, protect the colonocytes from external harm, and have anti-inflammatory and anti-carcinogenic effects. Moreover, studies using animal models for colonic disease found substantial positive effects of the use of SCFAs or dietary fiber on colonic health. Convincing human evidence is however lacking. Only a limited number of studies are of high quality; these are studies showing little or no favourable effect of SCFA treatment over placebo. This review underlines once more that it is important to execute human randomized controlled trials, since we cannot assume that effects from in vitro, ex vivo or animal studies can be directly translated into humans.

Chapter 7 sheds new light on the use of SCFAs in health and disease. The possibility to combine SCFAs with anti-inflammatory drug treatment such as mesalazine seems promising for the future..$^{62}$ In addition, the combination of SCFAs with probiotics or prebiotics, ${ }^{63}$ or even the combination of butyrate with a prebiotic plus mesalazine ${ }^{64}$ might be the clue for further optimizing treatment of e.g. inflammatory bowel disease. Butyrate-coated sutures might aid to mucosal healing after colonic surgery, as can be hypothesised from a study that showed better repair in tendon healing using butyric acid-impregnated sutures in rats. ${ }^{65}$ Overall, single SCFA treatment does not seem to be effective for treating colonic disease. However, there are a lot of options for SCFAs to further improve the efficacy of already established treatments. 


\section{CONCLUSION AND FUTURE PERSPECTIVES}

As discussed in this thesis and by others, actions and interventions that result in an increase in colonic and plasma SCFA concentrations could be used as a strategy not only to prevent and/or treat obesity and obesity-related insulin resistance, but also to positively affect colonic metabolic health. SCFAs are produced by microbial fermentation of indigestible fibers, i.e. prebiotics. Colonic SCFAs have local effects by establishing or maintaining colonic health. Moreover, SCFAs stimulate colonic GPR receptors, thereby exerting metabolic downstream signaling and increasing satiety by stimulating the secretion of the satiety hormones PYY and GLP-1. Importantly, colonic SCFAs can be absorbed and become systemically available for peripheral metabolic tissues such as muscle and adipose tissue where they improve substrate metabolism e.g. by favoring fat oxidation. Based on the outcomes of the present work described in this thesis, together with available evidence in literature, several suggestions for future studies can be made.

We found no metabolic effects after proximal compared with distal colonic acetate administration. We assume that after proximal colonic acetate administration the liver scavenges part of the SCFAs, which is not the case after distal colonic acetate suppletion. For future research, it will be interesting to investigate in more detail the exact fate of colonic SCFAs delivered in the proximal versus the distal colon. We suggest to use the set-up as proposed by Boets et al. ${ }^{20}$, delivering isotopically labeled SCFAs into the proximal and distal colon by specifically designed capsules or a fermentable prebiotic as vector to investigate the exact amounts of SCFAs that are released from the colon and integrated into several body compartments and metabolic pathways.

As we have shown that increasing distal colonic acetate exerted beneficial effects on human metabolism, the use of slow-fermentable dietary fibers increasing acetate in the distal part of the colon would be an interesting approach to investigate metabolic effects on peripheral tissues such as adipose tissue and muscle. The use of a slow fermentable dietary fiber as a vector to release SCFAs in the distal part of the colon seems a promising option. We propose to set up an explorative study to investigate if such a fiber is indeed fermented in the distal part of the colon. By performing a study similar to the one described in Chapter 4, using an isotopically labeled prebiotic, the exact time point of fermentation can be determined by measuring ${ }^{13} \mathrm{CO}_{2}$ excretion in exhaled air and plasma ${ }^{13} \mathrm{C}$-labeled SCFAs. If it is confirmed that this slow-fermentable prebiotic is fermented in the distal part of the colon, we would suggest to set up a longer-term human intervention to study the exact metabolic effects of the prebiotic intake. To investigate the possible stimulation of SCFA receptor expression in the distal part of the colon, colonic tissue samples should be taken to investigate the role of SCFA receptors and the ability of SCFAs to stimulate GPR expression in a specific part of the colon. 
Overall, although in vitro and animal studies show convincing effects of SCFAs on metabolism, more clinical research is needed to confirm these findings in humans. Colonic tissue from human volunteers could be used in ex vivo studies to confirm the metabolic effects of SCFAs found in in vitro or animal studies. In addition, it will be interesting to expose this human tissue to several conditions (i.e. SCFA composition or concentrations, microbial composition, pH) and/or SCFA receptor agonists or antagonists to investigate specific metabolic effects at the gut level. Ideally, colonic biopsies from different locations throughout the colon should be used to investigate differential effects throughout the colon to optimize treatment. Moreover, human intervention studies should focus on a specific target group, i.e. obese or prediabetic patients, as microbial composition and metabolic response differ between healthy and obese subjects. As we want to prevent that obese and/or prediabetic subjects develop full-blown type 2 diabetes with devastating complications, this specific group should be targeted in the studies suggested above and in future metabolic studies.

A patient-specific treatment, driven by a specific microbial response, may be an important future perspective. Since every person has its own microbial composition, it is possible that every person reacts differently to treatment or diet. As already described, this approach is still in its infancy and faces a lot of challenges and limitations. It will be interesting to set up a prospective cohort in which individuals are selected based on a specific feature in their gut microbiome, for example by Prevotella abundance, to test the hypothesis that a difference in microbial composition would lead to a different metabolic response to a dietary intervention. This approach of personalized medicine based on microbial composition could also be used in patients with colonic diseases, as a study on colonic SCFA application in patients with inflammatory bowel disease reported specific responders and non-responders to treatment. ${ }^{66}$

Moreover, we have reported that there are several options available to increase effectiveness of SCFA application in colonic health and disease. It will be interesting to investigate the combination of SCFAs with other medications or approaches to affect colonic health. For instance in inflammatory bowel disease, the combination of SCFAs with established medication such as mesalazine would be an interesting approach. Moreover, the use of butyrate-coated sutures in colonic surgery could lead to a better wound healing in patients. 


\section{REFERENCES}

1. Delzenne NM, Cani PD. A place for dietary fibre in the management of the metabolic syndrome. Current opinion in clinical nutrition and metabolic care 2005;8:636-40.

2. Cani PD, Lecourt E, Dewulf EM, et al. Gut microbiota fermentation of prebiotics increases satietogenic and incretin gut peptide production with consequences for appetite sensation and glucose response after a meal. The American journal of clinical nutrition 2009;90:1236-43.

3. Parnell JA, Reimer RA. Weight loss during oligofructose supplementation is associated with decreased ghrelin and increased peptide $Y Y$ in overweight and obese adults. The American journal of clinical nutrition 2009;89:1751-9.

4. Canfora EE, Jocken JW, Blaak EE. Short-chain fatty acids in control of body weight and insulin sensitivity. Nat Rev Endocrinol 2015.

5. Tolhurst $\mathrm{G}$, Heffron H, Lam YS, et al. Short-chain fatty acids stimulate glucagon-like peptide-1 secretion via the G-protein-coupled receptor FFAR2. Diabetes 2012;61:364-71.

6. den Besten G, Bleeker A, Gerding A, et al. Short-Chain Fatty Acids Protect Against High-Fat Diet-Induced Obesity via a PPARgamma-Dependent Switch From Lipogenesis to Fat Oxidation. Diabetes 2015;64:2398-408.

7. Perry RJ, Peng $L$, Barry NA, et al. Acetate mediates a microbiome-brain-beta-cell axis to promote metabolic syndrome. Nature 2016;534:213-7.

8. Cummings JH, Pomare EW, Branch WJ, et al. Short chain fatty acids in human large intestine, portal, hepatic and venous blood. Gut 1987;28:1221-7.

9. Todesco T, Rao AV, Bosello O, et al. Propionate lowers blood glucose and alters lipid metabolism in healthy subjects. The American journal of clinical nutrition 1991;54:860-5.

10. Venter CS, Vorster HH, Cummings JH. Effects of dietary propionate on carbohydrate and lipid metabolism in healthy volunteers. The American journal of gastroenterology 1990;85:54953.

11. Crouse JR, Gerson CD, DeCarli LM, et al. Role of acetate in the reduction of plasma free fatty acids produced by ethanol in man. Journal of lipid research 1968;9:509-12.

12. Laurent C, Simoneau C, Marks $L$, et al. Effect of acetate and propionate on fasting hepatic glucose production in humans. European journal of clinical nutrition 1995;49:484-91.

13. Freeland KR, Wolever TM. Acute effects of intravenous and rectal acetate on glucagon-like peptide-1, peptide YY, ghrelin, adiponectin and tumour necrosis factor-alpha. The British journal of nutrition 2010;103:460-6.

14. Fernandes J, Vogt J, Wolever TM. Intravenous acetate elicits a greater free fatty acid rebound in normal than hyperinsulinaemic humans. European journal of clinical nutrition 2012;66:1029-34.

15. Akanji AO, Bruce MA, Frayn KN. Effect of acetate infusion on energy expenditure and substrate oxidation rates in non-diabetic and diabetic subjects. European journal of clinical nutrition 1989;43:107-15.

16. Wolever TM, Brighenti F, Royall D, et al. Effect of rectal infusion of short chain fatty acids in human subjects. The American journal of gastroenterology 1989;84:1027-33.

17. Wolever TM, Spadafora P, Eshuis H. Interaction between colonic acetate and propionate in humans. Am J Clin Nutr 1991;53:681-7. 
18. Kaji I, Karaki S, Tanaka R, et al. Density distribution of free fatty acid receptor 2 (FFA2)-expressing and GLP-1-producing enteroendocrine L cells in human and rat lower intestine, and increased cell numbers after ingestion of fructo-oligosaccharide. Journal of molecular histology 2011;42:27-38.

19. Nohr MK, Pedersen MH, Gille A, et al. GPR41/FFAR3 and GPR43/FFAR2 as cosensors for short-chain fatty acids in enteroendocrine cells vs FFAR3 in enteric neurons and FFAR2 in enteric leukocytes. Endocrinology 2013;154:3552-64.

20. Boets E, Gomand SV, Deroover $L$, et al. Systemic availability and metabolism of colonic-derived short-chain fatty acids in healthy subjects: a stable isotope study. The Journal of physiology 2017;595:541-555.

21. Chourasia MK, Jain SK. Pharmaceutical approaches to colon targeted drug delivery systems. Journal of pharmacy \& pharmaceutical sciences : a publication of the Canadian Society for Pharmaceutical Sciences, Societe canadienne des sciences pharmaceutiques 2003;6:33-66.

22. Chambers ES, Viardot A, Psichas A, et al. Effects of targeted delivery of propionate to the human colon on appetite regulation, body weight maintenance and adiposity in overweight adults. Gut 2015;64:1744-54.

23. Aguirre M, Bussolo de Souza C, Venema K. The Gut Microbiota from Lean and Obese Subjects Contribute Differently to the Fermentation of Arabinogalactan and Inulin. PloS one 2016;11:e0159236.

24. Cani PD, Hoste $S$, Guiot $Y$, et al. Dietary non-digestible carbohydrates promote L-cell differentiation in the proximal colon of rats. The British journal of nutrition 2007;98:32-7.

25. Maslowski KM, Vieira AT, Ng A, et al. Regulation of inflammatory responses by gut microbiota and chemoattractant receptor GPR43. Nature 2009;461:1282-6.

26. Sina C, Gavrilova O, Forster M, et al. G protein-coupled receptor 43 is essential for neutrophil recruitment during intestinal inflammation. J Immunol 2009;183:7514-22.

27. Kim MH, Kang SG, Park JH, et al. Short-chain fatty acids activate GPR41 and GPR43 on intestinal epithelial cells to promote inflammatory responses in mice. Gastroenterology 2013;145:396-406 e1-10.

28. Smith PM, Howitt MR, Panikov N, et al. The microbial metabolites, short-chain fatty acids, regulate colonic Treg cell homeostasis. Science 2013;341:569-73.

29. Masui R, Sasaki M, Funaki Y, et al. G protein-coupled receptor 43 moderates gut inflammation through cytokine regulation from mononuclear cells. Inflamm Bowel Dis 2013;19:2848-56.

30. Ang Z, Er JZ, Tan NS, et al. Human and mouse monocytes display distinct signalling and cytokine profiles upon stimulation with FFAR2/FFAR3 short-chain fatty acid receptor agonists. Scientific reports 2016;6:34145.

31. Seok J, Warren HS, Cuenca AG, et al. Genomic responses in mouse models poorly mimic human inflammatory diseases. Proceedings of the National Academy of Sciences of the United States of America 2013;110:3507-12.

32. Pourghassem Gargari B, Dehghan P, Aliasgharzadeh A, et al. Effects of high performance inulin supplementation on glycemic control and antioxidant status in women with type 2 diabetes. Diabetes Metab J 2013;37:140-8.

33. Letexier D, Diraison F, Beylot M. Addition of inulin to a moderately high-carbohydrate diet reduces hepatic lipogenesis and plasma triacylglycerol concentrations in humans. The American journal of clinical nutrition 2003;77:559-64.

34. Russo F, Chimienti G, Riezzo G, et al. Inulin-enriched pasta affects lipid profile and Lp(a) concentrations in Italian young healthy male volunteers. European journal of nutrition 2008;47:453-9. 
35. Russo F, Clemente $C$, Linsalata $M$, et al. Effects of a diet with inulin-enriched pasta on gut peptides and gastric emptying rates in healthy young volunteers. European journal of nutrition 2011;50:271-7.

36. Brighenti F, Casiraghi MC, Canzi E, et al. Effect of consumption of a ready-to-eat breakfast cereal containing inulin on the intestinal milieu and blood lipids in healthy male volunteers. Eur J Clin Nutr 1999;53:726-33.

37. Jackson KG, Taylor GR, Clohessy AM, et al. The effect of the daily intake of inulin on fasting lipid, insulin and glucose concentrations in middle-aged men and women. The British journal of nutrition 1999;82:23-30.

38. Davidson MH, Maki KC. Effects of dietary inulin on serum lipids. J Nutr 1999;129:1474S-7S.

39. Russo F, Riezzo G, Chiloiro M, et al. Metabolic effects of a diet with inulin-enriched pasta in healthy young volunteers. Current pharmaceutical design 2010;16:825-31.

40. Vulevic J, Drakoularakou A, Yaqoob P, et al. Modulation of the fecal microflora profile and immune function by a novel trans-galactooligosaccharide mixture (B-GOS) in healthy elderly volunteers. The American journal of clinical nutrition 2008;88:1438-46.

41. Vulevic J, Juric A, Tzortzis G, et al. A mixture of trans-galactooligosaccharides reduces markers of metabolic syndrome and modulates the fecal microbiota and immune function of overweight adults. The Journal of nutrition 2013;143:324-31.

42. Roberfroid MB, Delzenne NM. Dietary fructans. Annu Rev Nutr 1998;18:117-43.

43. Timm DA, Stewart ML, Hospattankar A, et al. Wheat dextrin, psyllium, and inulin produce distinct fermentation patterns, gas volumes, and short-chain fatty acid profiles in vitro. J Med Food 2010;13:961-6.

44. Kaur A, Rose DJ, Rumpagaporn P, et al. In vitro batch fecal fermentation comparison of gas and short-chain fatty acid production using "slowly fermentable" dietary fibers. Journal of food science 2011;76:H137-42.

45. Wang X, Gibson GR. Effects of the in vitro fermentation of oligofructose and inulin by bacteria growing in the human large intestine. J Appl Bacteriol 1993;75:373-80.

46. Maathuis AJ, van den Heuvel EG, Schoterman MH, et al. Galacto-oligosaccharides have prebiotic activity in a dynamic in vitro colon model using a (13)C-labeling technique. The Journal of nutrition 2012;142:1205-12.

47. Hughes SA, Shewry PR, Li L, et al. In vitro fermentation by human fecal microflora of wheat arabinoxylans. Journal of agricultural and food chemistry 2007;55:4589-95.

48. Frost G, Sleeth ML, Sahuri-Arisoylu M, et al. The short-chain fatty acid acetate reduces appetite via a central homeostatic mechanism. Nature communications 2014;5:3611.

49. David LA, Maurice CF, Carmody RN, et al. Diet rapidly and reproducibly alters the human gut microbiome. Nature 2014;505:559-63.

50. Reijnders D, Goossens GH, Hermes GD, et al. Effects of Gut Microbiota Manipulation by Antibiotics on Host Metabolism in Obese Humans: A Randomized Double-Blind Placebo-Controlled Trial. Cell metabolism 2016;24:63-74.

51. Zmora N, Zeevi D, Korem T, et al. Taking it Personally: Personalized Utilization of the Human Microbiome in Health and Disease. Cell host \& microbe 2016;19:12-20.

52. Zeevi D, Korem T, Zmora N, et al. Personalized Nutrition by Prediction of Glycemic Responses. Cell 2015;163:1079-94.

53. Kovatcheva-Datchary P, Nilsson A, Akrami R, et al. Dietary Fiber-Induced Improvement in Glucose Metabolism Is Associated with Increased Abundance of Prevotella. Cell metabolism 2015;22:971-82. 
54. de la Cuesta-Zuluaga J, Mueller NT, Corrales-Agudelo V, et al. Metformin Is Associated With Higher Relative Abundance of Mucin-Degrading Akkermansia muciniphila and Several ShortChain Fatty Acid-Producing Microbiota in the Gut. Diabetes care 2017;40:54-62.

55. Claesson MJ, Jeffery IB, Conde S, et al. Gut microbiota composition correlates with diet and health in the elderly. Nature 2012;488:178-84.

56. Barton W, Penney NC, Cronin O, et al. The microbiome of professional athletes differs from that of more sedentary subjects in composition and particularly at the functional metabolic level. Gut 2017.

57. Thaiss CA, Zeevi $D$, Levy $M$, et al. Transkingdom control of microbiota diurnal oscillations promotes metabolic homeostasis. Cell 2014;159:514-29.

58. Wong JM, de Souza R, Kendall CW, et al. Colonic health: fermentation and short chain fatty acids. J Clin Gastroenterol 2006;40:235-43.

59. Bloemen JG, Venema $K$, van de Poll MC, et al. Short chain fatty acids exchange across the gut and liver in humans measured at surgery. Clin Nutr 2009;28:657-61.

60. Blau CA, Constantoulakis P, Shaw CM, et al. Fetal hemoglobin induction with butyric acid: efficacy and toxicity. Blood 1993;81:529-37.

61. Sher GD, Ginder GD, Little J, et al. Extended therapy with intravenous arginine butyrate in patients with beta-hemoglobinopathies. The New England journal of medicine 1995;332:1606-10.

62. Vernia P, Monteleone G, Grandinetti G, et al. Combined oral sodium butyrate and mesalazine treatment compared to oral mesalazine alone in ulcerative colitis: randomized, double-blind, placebo-controlled pilot study. Dig Dis Sci 2000;45:976-81.

63. Moeinian M, Ghasemi-Niri SF, Mozaffari S, et al. Beneficial effect of butyrate, Lactobacillus casei and L-carnitine combination in preference to each in experimental colitis. World journal of gastroenterology 2014;20:10876-85.

64. Assisi RF. Combined butyric acid/mesalazine treatment in ulcerative colitis with mild-moderate activity. Results of a multicentre pilot study. Minerva Gastroenterol Dietol 2008;54:231-8.

65. Leek BT, Tasto JP, Tibor LM, et al. Augmentation of tendon healing with butyric acid-impregnated sutures: biomechanical evaluation in a rabbit model. AmJ Sports Med 2012;40:1762-71.

66. Scheppach W. Treatment of distal ulcerative colitis with short-chain fatty acid enemas. A placebo-controlled trial. German-Austrian SCFA Study Group. Dig Dis Sci 1996;41:2254-9. 


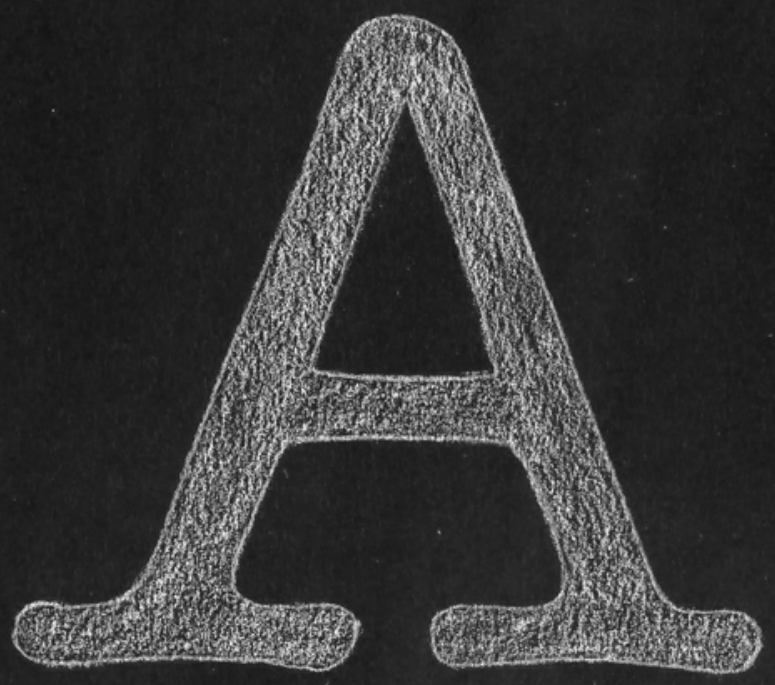


ADDENDUM

Summary

Samenvatting

Valorization

Curriculum vitae

List of publications

Dankwoord 



\section{SUMMARY}

In the last decade, it has become clear that gut microbiota play a prominent role in human colonic health and metabolism. Human gut microbiota have the ability to ferment otherwise indigestible dietary fibers into short-chain fatty acids (SCFAs), mostly acetate, propionate, and butyrate. These SCFAs have been suggested to contribute to the beneficial effects of fiber intake on colonic health as well as metabolic health. SCFAs influence several metabolic pathways, such as energy regulation, glucose homeostasis, lipid metabolism, and inflammation. Nonetheless, human evidence concerning the metabolic effects of SCFAs is scarce. The studies described in this thesis investigate the metabolic role of SCFAs and dietary fibers in overweight and obese human subjects, and advance our knowledge on SCFA receptors, and SCFA functioning and handling in the gut.

Chapter 2 describes a study in which the metabolic effects of colonic acetate infusions in overweight to obese men was investigated. Six subjects underwent two experimental periods: one with distal and one with proximal colonic acetate infusions. A feeding catheter was endoscopically positioned at the beginning of each period and remained in the colon for three consecutive days, enabling colonic acetate or placebo infusion during fasting conditions and after an oral glucose load. We demonstrated that distal colonic acetate infusion substantially increased fasting fat oxidation by $\sim 25 \%$. Moreover, distal colonic acetate infusions increased fasting plasma concentrations of the satiety hormone peptide YY (PYY). In addition, distal colonic acetate administration tended to increase fasting circulating acetate concentrations and to decrease concentrations of the pro-inflammatory cytokine TNF-a, but these effects were not significant. In contrast, proximal acetate administration did not affect human metabolism. These observations suggest that modulating colonic acetate in the distal part of the colon may be an important nutritional or therapeutic target to beneficially influence human metabolism.

Several studies have found that the ingestion of dietary fibers, i.e. prebiotics, has important metabolic effects. However, no studies have investigated if prebiotic-derived SCFAs contribute to the effects of prebiotics on human metabolism. Chapter 3 describes the acute metabolic effects of the prebiotic inulin. Stable isotope tracer methodology was used to study if these effects could be attributed to SCFA formation in overweight to obese subjects. In a crossover design, 14 healthy, overweight men consumed a high-fat milkshake containing either $24 \mathrm{~g}$ inulin, of which $0.5 \mathrm{~g}$ was $\mathrm{U}^{-{ }^{13} \mathrm{C}-l a b e l e d}$ inulin, or $24 \mathrm{~g}$ of the placebo maltodextrin. We found that the fermentable inulin increased early postprandial fat oxidation and reduced the early postprandial decrease in free fatty acid (FFA) concentrations when compared with the digestible placebo maltodextrin. Moreover, inulin ingestion resulted in lower postprandial glucose and insulin concentrations compared with maltodextrin. Importantly, we found that the ingestion of inulin but not maltodextrin 
increased plasma acetate concentrations. Tracer methodology revealed that inulin was fermented into all three SCFAs, as indicated by the appearance of ${ }^{13} \mathrm{C}$-acetate, ${ }^{13} \mathrm{C}$-butyrate, and ${ }^{13} \mathrm{C}$-propionate in feces and blood.

To investigate whether the acute metabolic effects translate into long-term clinical effects, a twelve-week randomized controlled trial was conducted in prediabetic subjects which assessed metabolic effects as well as effects of prebiotic supplementation on gut microbiota in Chapter 4. Forty-four prediabetic men and women were randomly assigned to ingest either $15 \mathrm{~g}$ of the prebiotic galacto-oligosaccharides (GOS) or an isocaloric placebo daily for twelve weeks. This study demonstrated that 12 weeks of GOS supplementation markedly increased the abundance of fecal Bifidobacterium spp. Surprisingly, this specific bifidogenic effect did not translate into alterations in fecal or plasma SCFA concentrations, systemic concentrations of gut-derived hormones, or systemic inflammatory markers. In addition, no significant changes in peripheral and adipose tissue insulin sensitivity, body composition, energy and substrate metabolism, and circulating metabolites were observed.

The observation that distal but not proximal acetate supplementation affected human metabolism (chapter 2) might suggest a differential expression pattern of the SCFA receptors. Therefore, mRNA expression of GPR41, GPR43, and GPR109a as well as expression of the satiety hormones PYY and proglucagon, as a precursor for GLP-1, was investigated throughout the human ileum and colon in Chapter 5. Intestinal biopsies were obtained from 16 healthy subjects undergoing colonoscopy. We observed that expression of GPR41, GRP43, and GPR109a, as well as PYY and proglucagon was highest in the ileum compared with the colon. In addition, GPR41 expression was higher in the proximal compared with distal colon. This seems contradictory to the results observed in chapter 2, as we only found metabolic effects after distal acetate infusion, while SCFA receptor expression appeared to be lower in that part of the gut. This can be explained by the fact that SCFA receptor activation is not the only mechanism by which SCFAs influence metabolism and that receptor expression can be stimulated by ingesting dietary fibers. The result of this study suggests that future studies specifically aiming at stimulating SCFA receptors should make use of easily fermentable fibers that increase SCFA production in the ileum and proximal colon.

In contrast to acetate and propionate, which are thought to have metabolic effects mostly on peripheral tissues such as muscle and adipose tissue, butyrate is considered most important for maintaining and improving colonic health, primarily by acting as an energy source for colonocytes. As high systemic concentrations of butyrate can cause side effects such as hypokalemia and nausea, the inter-organ trafficking of a butyrate enema was investigated in Chapter 6 in a surgical setting. Twelve patients undergoing upper abdominal surgery were included in this randomized controlled trial. During surgery, one group received a butyrate enema and the other group a placebo enema. Plasma samples 
were taken from the radial artery, hepatic vein, and portal vein, thereby providing the opportunity to analyze SCFA concentrations and calculate fluxes over the portal-drained viscera, liver, and splanchnic area. We showed an increased release of butyrate from the gut shortly after rectal butyrate administration combined with an increased butyrate uptake by the liver. As a result, there was no splanchnic release of butyrate in these subjects, suggesting that the liver is capable of metabolizing rectally administered butyrate to prevent release into the systemic circulation.

Chapter 7 reviews the effects of SCFA enemas in colonic diseases, i.e. colonic inflammation, carcinogenesis, and mucosal protection and healing. In vitro and ex vivo studies show that SCFAs have anti-inflammatory and anti-carcinogenic effects, play an important role in maintaining metabolic homeostasis in colonocytes, and protect colonocytes from external harm. Animal studies have found substantial positive effects of SCFAs or dietary fiber intake on colonic disease, but convincing human evidence is lacking. The limited number of human intervention studies published to date show little or no favorable effects of SCFA treatment over placebo. Therefore, we suggest follow-up research should focus on using combination therapies of SCFAs with anti-inflammatory drugs, prebiotics, or probiotics in the case of colonic inflammation. In addition, the use of 'prodrugs', a biologically inactive compound that can be metabolized in the body to produce SCFAs could be useful for anti-carcinogenic therapy. Moreover, direct administration of SCFAs via surgical sutures could improve mucosal healing after colonic surgery.

In Chapter 8, the main findings of this thesis are described and integrated. Moreover, new insights and future perspectives based on these findings are discussed. In summary, actions and interventions that result in an increase in colonic and plasma SCFA concentrations could be used as a strategy not only to prevent and/or treat obesity and obesity-related insulin resistance, but also to positively affect colonic metabolic health. Although in vitro and animal studies show convincing effects of SCFAs on metabolism, these results can only be confirmed to a limited extent in human subjects so far, and thus more clinical research is needed to investigate these effects in humans. A patient-specific treatment, driven by a specific microbial response, may be an important future perspective in this case. 



\section{SAMENVATTING}

Het afgelopen decennium is duidelijk geworden dat darmbacteriën een prominente rol spelen in darmgezondheid en stofwisseling van de mens. Darmbacteriën hebben het vermogen om onverteerbare voedingsvezels te fermenteren in zogenaamde korte-ketenvetzuren, met name acetaat (azijnzuur), propionaat (propionzuur) en butyraat (boterzuur). Deze korte-ketenvetzuren dragen mogelijk bij aan de gunstige effecten van vezelinname op zowel de gezondheid van de darm als de stofwisseling in het menselijke lichaam, oftewel het humane metabolisme. Korte-ketenvetzuren hebben invloed op verschillende processen in het lichaam, zoals energieregulatie, suikerhuishouding en vetmetabolisme, en kunnen de mate van ontsteking verminderen. Wetenschappelijk bewijs voor de metabole effecten van korte-ketenvetzuren in mensen is echter schaars. In dit proefschrift is een aantal studies beschreven warin de effecten van korte-ketenvetzuren en voedingsvezels op het metabolisme van personen met overgewicht en obesitas bestudeerd werden. Verder werd onderzoek gedaan naar de mogelijke effecten van korte-ketenvetzuren op darmniveau en werden betrokken receptoren in kaart gebracht in de humane darm.

Hoofdstuk 2 beschrijft een studie waarin de metabole effecten van infusie van het korte-ketenvetzuur acetaat in de darm van overgewichtige mensen onderzocht werd. Zes mannen met overgewicht of obesitas ondergingen twee experimentele perioden: één waarbij acetaat werd geïnfundeerd in het distale deel van het colon (dicht bij rectum en anus) en één waarbij acetaat werd geïnfundeerd in het proximale deel van het colon (dicht bij de dunne darm). Aan het begin van elke periode werd endoscopisch een darmkatheter ingebracht, die gedurende drie opeenvolgende testdagen in de dikke darm bleef. Via de darmkatheter werd acetaat of een niet-werkzame placebo geïnfundeerd in één van de locaties in de dikke darm. Het onderzoek toonde aan dat acetaatinfusie in het distale deel van het colon de vetverbranding (vetoxidatie) met ongeveer $25 \%$ liet toenemen. Bovendien verhoogde acetaatinfusie in het distale deel van het colon de concentraties van het verzadigingshormoon peptide YY (PYY) in de circulatie in gevaste toestand. Daarnaast had de toediening van acetaat in het distale deel van het colon de neiging om de acetaatconcentraties in het bloed te verhogen en de concentraties van de ontstekingsparameter TNF-a te verlagen, doch deze effecten waren niet significant. Acetaattoediening in het proximale deel van het colon had geen invloed op het humane metabolisme. Deze bevindingen suggereren dat verhoging van het korte-ketenvetzuur acetaat in het distale deel van de dikke darm een goede strategie kan zijn om de menselijke stofwisseling te verbeteren.

Onderzoek heeft aangetoond dat inname van voedingsvezels, ook wel prebiotica genoemd, het humane metabolisme kan beïnvloeden. Het is echter niet bekend of korte-ketenvetzuren hierbij een rol spelen. In Hoofdstuk 3 worden de acute effecten van het prebioticum inuline op de stofwisseling in proefpersonen met overgewicht of obesitas 
beschreven. Hierbij werd gebruik gemaakt van stabiele-isotopen-tracermethodologie om te onderzoeken of deze effecten kunnen worden toegeschreven aan de vorming van korte-ketenvetzuren. Veertien gezonde mannen met overgewicht consumeerden een milkshake met daarin 24 gram inuline, waarvan 0,5 gram $U-{ }^{13} \mathrm{C}$-gelabeld inuline, of 24 gram van de placebo maltodextrine. Het ${ }^{13} \mathrm{C}$-label maakt het mogelijk om inuline in het lichaam te traceren. Bij fermentatie van inuline wordt het label overgedragen aan de korte-ketenvetzuren, waardoor vastgesteld kan worden of korte-ketenvetzuren gevormd worden uit inuline en in welke verhouding. Deze studie toonde aan dat, gedurende de eerste uren na inname, het fermenteerbare voedingsvezel inuline resulteerde in een verhoging van de vetoxidatie en een verminderde verlaging van de vrije vetzuurconcentraties in vergelijking met het verteerbare placebo maltodextrine. Bovendien veroorzaakte inuline-inname een verminderde postprandiale stijging in glucose- en insulineconcentraties in vergelijking met maltodextrine. Verder resulteerde inuline-inname in verhoogde acetaatconcentraties en verminderde vrije vetzuurconcentraties in de late postprandiale periode vergeleken met maltodextrine. Tracermethodologie toonde aan dat inuline gefermenteerd werd in alle drie de korte-ketenvetzuren, aangezien ${ }^{13} \mathrm{C}$-acetaat, ${ }^{13} \mathrm{C}$-butyraat en ${ }^{13} \mathrm{C}$-propionaat detecteerbaar waren in zowel ontlasting als bloed.

Om te onderzoeken of de acute metabole effecten vertaald kunnen worden naar gunstige langere termijn effecten, werd een studie uitgevoerd waarbij proefpersonen met een voorstadium van diabetes (prediabetes) het prebioticum galacto-oligosacchariden (GOS) hebben ingenomen gedurende een periode van 12 weken (Hoofdstuk 4). Vierenveertig mannen en vrouwen met prediabetes werden willekeurig toegewezen aan een groep die gedurende 12 weken dagelijks 15 gram GOS of een placebo met dezelfde calorische waarde consumeerde. Deze studie toonde aan dat 12 weken GOS-suppletie de bacteriesamenstelling in de darm aanzienlijk veranderde, met een verhoging van de Bifidobacterium spp. Dit bifidogeen effect vertaalde zich echter niet in veranderingen in korte-ketenvetzuurconcentraties in zowel ontlasting als bloed, noch leidde dit tot veranderingen in systemische concentraties van verzadigingshormonen en ontstekingsmarkers. Bovendien werden de insulinegevoeligheid, lichaamssamenstelling, het energie- en substraatmetabolisme en circulerende metabolieten niet significant beïnvloed door de vezelinname.

De bevinding dat acetaatinfusie in het distale maar niet in het proximale deel van het colon het humane metabolisme beïnvloedde, heeft ertoe geleid dat het expressiepatroon van de korte-ketenvetzuurreceptoren GPR41, GPR43 en GPR109a alsook van PYY en proglucagon, als voorganger van GLP-1, in verschillende locaties in de dunne en dikke darm nader onderzocht werd (Hoofdstuk 5). Darmbiopten werden verkregen van 16 gezonde proefpersonen die een reguliere colonoscopie ondergingen. We toonden aan dat de expressie van GPR41, GRP43 en GPR109a en de expressie van PYY en proglucagon hoger was in het ileum in vergelijking met de dikke darm. Bovendien was de expressie van GPR41 hoger in het proximale in vergelijking met het distale deel van het colon. Dit lijkt 
in tegenspraak te zijn met de uitkomsten van hoofdstuk 2, waarbij alleen distale infusie zorgde voor metabole effecten. Er zijn echter meerdere oorzaken die deze discrepantie kunnen verklaren. Mogelijk is activatie van de korte-ketenvetzuurreceptor niet de enige manier waarop korte-ketenvetzuren het metabolisme kunnen beïnvloeden. Daarnaast is het ook mogelijk dat receptorexpressie kan worden beïnvloed door de inname van voedingsvezels. Deze studie suggereert dat men gebruik zou moeten maken van makkelijk te fermenteren voedingsvezels, leidend tot een verhoging van korteketenvetzuren in het ileum en proximale colon, indien men effecten wil bekomen middels stimulering van de onderzochte receptoren.

Acetaat en propionaat hebben met name metabole effecten op perifere weefsels zoals spieren en vetweefsel, terwijl butyraat een belangrijke rol speelt bij de gezondheid van de darm. Butyraat dient als energiebron voor darmcellen. Echter, hoge systemische concentraties van butyraat kunnen bijwerkingen veroorzaken, zoals hypokaliëmie en misselijkheid. In Hoofdstuk 6 werd onderzocht in welke mate dat toediening van butyraat via een klysma in een chirurgische setting tot een verhoging van circulerende butyraatconcentraties leidde. Twaalf patiënten die een grote buikoperatie ondergingen, kregen tijdens de operatie een butyraat of placebo klysma. Bloedmonsters werden afgenomen uit een polsslagader (arteria radialis), de leverader en de poortader. Korte-ketenvetzuurconcentraties werden bepaald welke ons in staat stelden fluxen te berekenen. Deze fluxen dienen als kwantitatieve maat voor opname of afgifte van korte-ketenvetzuren door de darm, lever en het splanchnische gebied (maag, twaalfvingerige darm, dunne en dikke darm, galwegen, lever, alvleesklier en milt). Direct na rectale butyraattoediening was een verhoogde afgifte van butyraat door de darm zichtbaar in combinatie met een verhoogde butyraatopname door de lever. Als gevolg was er geen splanchnische afgifte van butyraat waarneembaar, wat suggereert dat de lever in staat is om het rectaal toegediende butyraat op te nemen waardoor het butyraat niet in het bloed terecht komt.

Hoofdstuk 7 beschrijft een overzicht van de literatuur over de effecten van korte-ketenvetzuurklysma's op darmziekten, darmkanker en het genezingsproces na darmchirurgie. Celstudies hebben aangetoond dat korte-ketenvetzuren een anti-inflammatoir en anti-carcinogeen effect hebben, een belangrijke rol spelen bij het behoud van metabole homeostase in dikkedarmcellen (colonocyten) en deze cellen beschermen tegen externe schade. Dierstudies hebben positieve effecten van korte-ketenvetzuren en voedingsvezels op de gezondheid van de dikke darm aangetoond. Er is echter weinig bekend over de effecten van korte-ketenvetzuurklysma's in de mens. Een beperkt aantal studies onderzocht het effect van korte-ketenvetzuurtoediening bij chronische darmontsteking, darmkanker en het genezingsproces na darmchirurgie in de mens. Deze studies laten weinig of geen gunstige effecten zien van deze behandeling ten opzichte van placebo. Daarom stellen we voor om vervolgonderzoek te richten op het gebruik van combinatietherapieën waarbij toediening van korte-ketenvetzuren wordt gecombineerd met het 
gebruik van ontstekingsremmende geneesmiddelen, prebiotica of probiotica in het geval van inflammatoire darmziekten. Het gebruik van zogenaamde 'prodrugs' die door omzetting in het lichaam korte-ketenvetzuren vrijgeven kan toegepast worden in de bestrijding van darmkanker en hechtingen die speciaal gecoat zijn met het korte-ketenvetzuur butyraat kunnen de mucosale genezing na de darmchirurgie te verbeteren.

In Hoofdstuk 8 worden de belangrijkste bevindingen van dit proefschrift bediscussieerd, alsook nieuwe inzichten en toekomstperspectieven. Samenvattend kunnen interventies die leiden tot een toename van korte-ketenvetzuurconcentraties in het colon en plasma mogelijk gebruikt worden als een strategie om obesitas en insulineresistentie te voorkomen en/of te behandelen, maar ook om de gezondheid van de darm op een positieve manier te beïnvloeden. Hoewel in vitro en dierstudies overtuigende effecten van korte-ketenvetzuren op metabolisme aantonen, kunnen deze resultaten tot nog toe slechts in beperkt mate in mensen worden bevestigd. Er is dus meer klinisch onderzoek nodig om de effecten bij mensen diepgaander te onderzoeken. Een patiënt-specifieke behandeling, werkend vanuit een specifieke samenstelling en functie van de darmbacteriën, kan daarbij een belangrijk toekomstperspectief zijn. 


\section{VALORIZATION}

"Valorization is the process of value creation out of knowledge by making this knowledge suitable and available for economic or societal utilization and to translate this into high-potential products, services, processes, and industrial activity." (Landelijke Commissie Valorisatie 2011:8)

The relationship between food intake and human health is complex. Food intake supplies nutrients that can be used for growth, repair, energy provision, and other functions. Food intake can also negatively influence health and contribute to the development of disease. Not only the lack of high-quality food in low-income countries, but also overnutrition in high-income countries emerges as a threat to public health. Overnutrition, i.e. total energy from food intake exceeding energy expenditure, can cause overweight and obesity on the long term. Overweight, obesity, and related disorders such as type 2 diabetes represent a rapidly growing health threat. In 2014, approximately 1.9 billion adults worldwide were overweight $\left(25<\mathrm{BMl}<30 \mathrm{~kg} / \mathrm{m}^{2}\right)$ and about 600 million were obese $\left(\mathrm{BMl}>30 \mathrm{~kg} / \mathrm{m}^{2}\right)$. It has been estimated that obesity and related disorders contribute to 2 - 7\% of total health care costs. As such, obesity imposes a tremendous economic burden on health care systems. ${ }^{1,2}$ Importantly, obesity increases the risk for type 2 diabetes, cardiovascular disease, and several types of cancer. As such, it has been suggested that growth of the obese population could lead to future declines in life expectancy. ${ }^{3}$ This thesis provides information that can be used to design intervention strategies that help to maintain a healthy body weight in order to prevent obesity-related disorders and subsequent impact on quality of life and healthcare systems.

Attempts have been made to fight obesity with the simple regimen: "Eat less, exercise more". However, the implementation of such a lifestyle intervention fails for the majority of people and will never solve the obesity epidemic. Therefore, better knowledge of human metabolism and the development of effective interventions to prevent the onset of overweight and obesity-related disorders may improve life expectancy and quality of life and reduce healthcare costs. In conclusion, the world is in dire need of effective and safe strategies enhancing energy expenditure, and fat oxidation in particular.

Studies performed over the last decades have indicated that intestinal microbiota composition and function are closely associated with health and thus with the development and progression of a variety of disorders. Several years ago, a class of compounds defined as prebiotics were recognized for their ability to manipulate gut microbiota composition and benefit the host. Especially the ability of gut microbiota to ferment otherwise indigestible food components, such as dietary fibers, into short-chain fatty acids (SCFAs) adds a unique function to the human body. In vitro and animal studies have shown that SCFAs are involved in energy regulation, lipid metabolism, glucose homeostasis, and inflammation. 
A highly promising intervention for preventing or treating metabolic diseases such as obesity and type 2 diabetes would therefore be a change in gut microbiota composition and function to benefit human health, i.e. to influence energy expenditure and fat oxidation in particular. Results from these mechanistic in vitro or animal studies cannot be directly translated into the human situation, because of different microbial composition in humans, and thus cannot simply be used to redefine public health guidelines. This thesis describes several clinical trials in human subjects and attempts to better understand the role of SCFAs in human metabolism.

The studies described in this thesis are derived from an intensive collaboration between academia, industry, and government with scientists in the field of human nutrition and physiology, clinicians, and health care professionals. The close collaboration between industry and academia leads to demand-driven research that not only helps to better understand physiology but also provides information that can be used to create or optimize product formulations and/or services. It is important that academia, government and industry collaborate as the government detects and controls health issues, academia has the ability to study these issues and gain novel information, which then can be used by industry to create and market new health products.

The present thesis has shown that SCFAs, especially acetate applied in the distal part of the colon, can serve as important nutritional and/or therapeutic targets to promote human metabolism. Increasing acetate concentrations in the distal part of the colon could theoretically be achieved by developing slow-fermentable dietary fibers that take a longer time to ferment and thus the fermentation process will take in the distal part of the colon. Moreover, a slow-fermentable fiber can be modified to serve as a vector that can bind acetate in order to further increase acetate concentrations. The challenge with this approach is the difference in gut transit time between individuals. Human gut transit time ranges from 2 - 5 h for gastric emptying, 2 - 6 h for small bowel transit, and 10 - 59 h for colonic transit. One can imagine that the wide range in colonic transit time makes it difficult to develop a 'one size fits all' slow-fermentable fiber. The same holds true for special coatings that resolve when in contact with microbiota or coatings that release their content after remaining in the gut for a specific amount of time. This challenge can be bypassed by using enemas. These are extremely handy in scientific settings; however, use in daily life is not feasible, as they should be used daily for several years or decades to prevent or treat diseases. Thus, more research on product development using the information above should be initiated.

The present thesis has shown that the prebiotic inulin is fermented into SCFAs, and thus might have an important nutritional implication for human health. In addition, replacing digestible fibers with fermentable fibers such as inulin attenuates the rise in blood glucose after a meal intake without affecting hunger and satiety. The lower glycemic index of inulin compared with digestible fibers also lowers caloric intake, and thus promotes 
weight loss. The ingestion of up to $24 \mathrm{~g}$ of inulin caused no side-effects in our study subjects and was easy to consume, and can thus be considered safe and feasible for daily intake. The formation of SCFAs in the colon might have additional health effects by further increasing fat oxidation.

Results from this thesis show that long-term supplementation of the prebiotic galacto-oligosaccharides (GOS) changes gut microbiota composition, but has no effect on metabolic outcomes. It is possible that microbial imbalance (also termed dysbiosis) in our obese prediabetic population might have hampered any beneficial metabolic effects. Microbial utilization of prebiotics can only occur if the appropriate species of bacteria are abundantly present in the host's microbiota. A number of environmental factors, including mode of delivery and early feeding, antibiotic use, disease status, adult diet, and metabolic status can influence the human gut microbiota composition and possibly the effect of prebiotic supplementation. Therefore, a patient-specific treatment to target a specific microbial response is an important aspect for future research. Microbiota profiling using state-of-the art techniques, such as Human Intestinal Tract Chip (HITChip), makes it possible to map the entire microbiome. To allow for personalized medicine in the future, we need to develop affordable and scalable techniques for mapping microbiota composition but also for microbiota function. Based on the unique microbiota composition and function, a specific prebiotic can be prescribed in order to effectively improve microbiota function and subsequent metabolic parameters.

SCFAs are important for maintaining or improving colonic health. Especially butyrate enemas have the potential for treating distal colonic diseases such as the inflammatory bowel disease ulcerative colitis. On the other hand, high systemic levels of butyrate can cause side effects such as hypokalemia and nausea. We have shown that the liver has a large capacity to catabolize colonically delivered butyrate, thereby preventing a rise in systemic butyrate and making the use of butyrate enemas safe as a treatment for colonic disease. This thesis also includes a review on the role of SCFAs in colonic inflammation, carcinogenesis, as well as mucosal protection and healing. In short, in vitro, ex vivo, and animal studies show promising results of SCFA treatment in colonic diseases, while only a limited number of human studies have been performed and show little or no favorable effects of direct SCFA application when compared with placebo. However, it is possible that SCFAs support and improve the efficacy of established medical treatment, such as the use of the first class anti-inflammatory drug mesalazine. Especially in ulcerative colitis it is easy to reach the colon and its mucosa by using SCFA enemas, because ulcerative colitis is characterized by superficial mucosal inflammation predominantly in the distal part of the colon. In addition it has been suggested that combining mesalazine treatment with SCFA enemas is an effective treatment to attack colonic inflammation. Also, the combination of SCFAs with probiotics or prebiotics, or even the combination of SCFAs, prebiotics, and mesalazine, might serve as an effective treatment for inflammatory bowel disease. SCFAs 
may also be used in colonic surgery, e.g. by coating surgical sutures with SCFAs to promote wound healing after surgery. Evidence on this topic is still scarce and these sutures have only been applied in animals, but the outcome of these studies are promising for future human studies.

The overwhelming load of information about healthy and unhealthy foods in the news and social media often provides the general public with confusing information and lifestyle advices. Frequently, only positive results and/or false translation of results find their way to the media, i.e. publication bias or industry bias may play a role. A recent analysis shows that industry funded randomized-controlled trials are seven times more likely to report a positive result in general and abdominal surgery than a non-industry-funded trial. ${ }^{4} \mathrm{~A}$ public-private partnership such as TIFN is an excellent way to fund research, as risk of publication and industry bias is diminished and data are not published in a selective way. As researchers, we should feel obliged to translate our scientific knowledge into practical guidelines, but also to highlight different aspects and negative results of our studies. Moreover, the concept of personalized medicine should be introduced to the general public, and it should be explained that a 'one size fits all' therapy does not exist. This thesis highlights that the gut microbiota and its fermentation products such as SCFAs play an important role in human metabolism, although we need to gather more information before patient-specific health recommendations can be made.

\section{REFERENCES}

1. Ng M, Fleming T, Robinson M, et al. Global, regional, and national prevalence of overweight and obesity in children and adults during 1980-2013: a systematic analysis for the Global Burden of Disease Study 2013. Lancet 2014;384:766-81.

2. World Health Organization. Fact Sheet Obesity and overweight, 2016.

3. Olshansky SJ, Passaro DJ, Hershow RC, et al. A potential decline in life expectancy in the United States in the 21st century. The New England journal of medicine 2005;352:1138-45.

4. Probst P, Knebel P, Grummich K, et al. Industry Bias in Randomized Controlled Trials in General and Abdominal Surgery: An Empirical Study. Annals of surgery 2016;264:87-92. 


\section{CURRICULUM VITAE}

Christina Maria (Kirsten) van der Beek was born on April 25, 1987 in Sittard, the Netherlands. After graduation from the Trevianum Gymnasium in Sittard in 2005, she started medical school at the Maastricht University, Faculty of Health, Medicine and Life Sciences. During medical school she finished clinical and scientific traineeships at the Department of Internal Medicine, Division of Gastroenterology and Hepatology, Maastricht University Medical Center. After graduating in 2011, Kirsten started as a PhD-fellow at the Department of Surgery and the Department Internal Medicine, Division of Gastroenterology and Hepatology within NUTRIM School of Nutrition and Translational Research in Metabolism and TI Food and Nutrition under the supervision of prof. dr. C.H.C Dejong, prof. dr. A.A.M. Masclee, and dr. K. Lenaerts, and in close collaboration with prof. dr. E.E. Blaak. During her PhD, she was a member of the board of the Pélerin Resident Symposium of Maastricht University Medical Center. At the $1^{\text {st }}$ of December 2015, she started her residency in Gastroenterology and Hepatology under supervision of prof. dr. A.A.M. Masclee. She is currently working at the Department of Internal Medicine at Viecuri Hospital in Venlo under supervision of dr. M.M.H. Hermans. 


\section{LIST OF PUBLICATIONS}

van der Beek CM, Dejong CHC, Troost FJ, Masclee AAM, Lenaerts K. Role of short-chain fatty acids in colonic inflammation, carcinogenesis, and mucosal protection and healing. Nutr Rev. 2017 Apr 1;75(4):286-305.

Canfora EE, van der Beek CM, Jocken JWE, Goossens GH, Holst JJ, Olde Damink SWM, Lenaerts K, Dejong CHC, Blaak EE. Colonic infusions of short-chain fatty acid mixtures promote energy metabolism in overweight/obese men: a randomized crossover trial. Sci Rep. 2017 May $24 ; 7(1): 2360$.

Canfora EE*, van der Beek CM*, Hermes GDA, Goossens GH, Jocken JWE, Holst JJ, van Eijk HM, Venema K, Smidt H, Zoetendal EG, Dejong CHC, Lenaerts K, Blaak EE. Supplementation of Diet With Galacto-oligosaccharides Increases Bifidobacteria, but Not Insulin Sensitivity, in Obese Prediabetic Individuals. Gastroenterology. 2017 Jul;153(1):87-97.e3.

* Shared first authors

van der Beek CM*, Canfora EE*, Lenaerts K, Troost FJ, Olde Damink SWM, Holst JJ, Masclee AA, Dejong CH, Blaak EE. Distal, not proximal, colonic acetate infusions promote fat oxidation and improve metabolic markers in overweight/obese men. Clin Sci (Lond). 2016 Nov 1;130(22):2073-2082.

* Shared first authors

Reijnders D, Goossens GH, Hermes GD, Neis EP, van der Beek CM, Most J, Holst JJ, Lenaerts K, Kootte RS, Nieuwdorp M, Groen AK, Olde Damink SW, Boekschoten MV, Smidt H, Zoetendal EG, Dejong CH, Blaak EE. Effects of Gut Microbiota Manipulation by Antibiotics on Host Metabolism in Obese Humans: A Randomized Double-Blind Placebo-Controlled Trial. Cell Metab. 2016 Jul 12;24(1):63-74.

van der Beek CM*, Bloemen JG*, van den Broek MA, Lenaerts K, Venema K, Buurman WA, Dejong CH. Hepatic Uptake of Rectally Administered Butyrate Prevents an Increase in Systemic Butyrate Concentrations in Humans. J Nutr. 2015 Sep;145(9):2019-24.

* Shared first authors 


\section{MANUSCRIPTS READY FOR SUBMISSION}

van der Beek CM, Canfora EE, Kip AM, Gorissen SHM, Olde Damink SWM, van Eijk HM, Holst JJ, Blaak EE, Dejong CHC, Lenaerts K. The prebiotic inulin improves substrate metabolism and promotes short-chain fatty acid production in overweight to obese men.

van der Beek CM, van Avesaat M, van der Wielen N, Kip AM, Troost FJ, Masclee AAM, Dejong CHC, Lenaerts K. Expression of short-chain fatty acid receptors GPR41, GPR43, and GPR109a is higher in the human ileum and proximal colon compared with the distal colon.

\section{ORAL PRESENTATIONS}

"The Prebiotic Inulin Enhances Fat Oxidation and Improves Metabolic Parameters in Overweight Males" Digestive Disease Week (DDW), Washington DC, USA, 2015

"Expression of the Short-Chain Fatty Acid Receptors GPR41 and GPR43 Throughout the Human Ileum and Colon" Digestive Disease Week (DDW), Washington DC, USA, 2015

"The Prebiotic Inulin Enhances Fat Oxidation and Improves Metabolic Parameters in Overweight Males" Netherlands Society for Parenteraland Enteral Nutrition (NESPEN) meeting during Digestive Disease Days, Veldhoven, the Nederlands, 2015

"Distal versus proximal colonic acetate infusions differentially affect human metabolism" Digestive Disease Days, Veldhoven, the Netherlands, 2014

"Colonic acetate infusions promote fat oxidation and improve metabolic parameters in overweight males" Diabetes and Metabolism symposium, Düsseldorf, Germany, 2014

"Colonic acetate infusions promote fat oxidation and improve metabolic parameters in overweight males". European Society for Clinical Nutrition and Metabolism (ESPEN), Geneva, Switzerland, 2014 


\section{POSTER PRESENTATIONS}

"The Prebiotic Inulin Enhances Fat Oxidation and Improves Metabolic Parameters in Overweight Males" European Society for Clinical Nutrition and Metabolism (ESPEN), Lisbon, Portugal, 2015

"Expression of the Short-Chain Fatty Acid Receptors GPR41 and GPR43 Throughout the Human Ileum and Colon". Digestive Disease Days, Veldhoven, the Netherlands, 2015.

\section{AWARDS}

NESPEN Abstract prize 2015: "The Prebiotic Inulin Enhances Fat Oxidation and Improves Metabolic Parameters in Overweight Males"

DDW Early Stage Investigator award 2015: "The Prebiotic Inulin Enhances Fat Oxidation and Improves Metabolic Parameters in Overweight Males" 


\section{DANKWOORD}

Een proefschrift is natuurlijk niet compleet zonder dankwoord, en ik heb het geluk gehad dat ik met ontzettend veel mensen de afgelopen paar jaar heb mogen samenwerken. Het blijft natuurlijk altijd tricky om iedereen te benoemen, maar ik ga een poging wagen...

Professor Dejong, beste Kees. Ik kan me nog goed het moment herinneren dat we elkaar ontmoetten toen ik bij jou op gesprek kwam over een eventuele aanstelling als PhD-studente. Jij gaf gelijk aan dat je graag getutoyeerd wilde worden en uiteindelijk hebben we lang met elkaar gekletst. Je gaf me daarmee meteen een vertrouwd gevoel. Dat tutoyeren ben ik door de jaren blijven doen en je verhalen zijn ook niet minder sterk geworden. Mijn manuscripten werden met de hand nagekeken en ik moest soms goed mijn best doen om te ontcijferen wat er precies geschreven stond. Vaak schreef je er nog een grapje of een complimentje bij en dat maakte mijn hele dag goed. Kees, hartelijk dank voor je steun de afgelopen jaren, voor jouw wetenschappelijke inbreng, maar ook je warme persoonlijkheid en het gevoel dat de deur altijd voor me open stond in goede en in minder goede tijden.

Professor Masclee, beste Ad. Het kwam door uw aanbeveling dat ik uiteindelijk in dit fantastische promotietraject ben gerold en daar ben ik u heel dankbaar voor. Toen ik aan het einde van mijn semi-stage aangaf dat ik graag eerst fulltime onderzoek wilde doen voordat ik in de kliniek wilde werken, was u gelijk enthousiast en werd ik in sneltreinvaart langs Kees en Ellen geleid. Ik was er zelf nauwelijks van op de hoogte dat ik van een simpel gesprekje in een soort sollicitatiegesprek rolde, tot ik aan het einde van de dag een mail kreeg met de tekst "welkom in de groep". Bedankt voor de afgelopen jaren, waar ik altijd welkom was en ik veel wetenschappelijke input kreeg. Ik kijk uit naar een mooie gezamenlijke toekomst binnen mijn opleiding tot MDL-arts.

Beste Kaatje, de afgelopen jaren was jij mijn rots in de branding en heb ik je met allerlei kleine en grote, belangrijke en minder belangrijke, werk-gerelateerde en niet-werk-gerelateerde dingen gestalkt. Zelf moest je vaak ook meezoeken naar bepaalde oplossingen of hielp je me met praktische zaken regelen en zo kwamen we er altijd samen uit. Manuscripten gingen eerst een aantal keren tussen ons op en neer voordat ze naar Kees en Ad gingen en het kwam geregeld voor dat we elkaar minstens 10-20 keer per dag mailden. Gelukkig was er ook altijd tijd voor een sociaal praatje en hebben we ook menige avonden niet over het werk gepraat. Ik heb je de afgelopen jaren zien groeien als mentor en wetenschapper en ik denk dat jij nog veel gaat bereiken de komende tijd! Heel erg bedankt dat je er altijd voor me was. 
Ik wil ook graag mijn beoordelingscommissie, bestaande uit prof. Nicole Bouvy, dr. Daisy Jonkers, prof. Max Nieuwdorp, prof. Jogchum Plat en prof. Kristin Verbeke bedanken voor de positieve beoordeling van mijn proefschrift en voor jullie deelname in de corona. Prof. Bert Groen, bedankt dat u bereid bent plaats te nemen in de corona.

Ik wil bij deze alle TIFN partners, alsook alle leden van de TIFN onderzoeksgroep GH003, bedanken voor hun tijd, energie en adviezen om ons onderzoek naar een hoger niveau te tillen.

De onderzoekswereld is niets zonder proefpersonen en daarom wil ik hier ruimte maken om al mijn proefpersonen te bedanken voor hun tijd en inzet om dit onderzoek mogelijk te maken.

Beste Ralph, wij kennen elkaar natuurlijk al jaren en waren goed bevriend voordat we allebei als promovendus bij de chirurgie aan de slag gingen. Ik als GE-meisje, jij als één van de vaatboys. Enige minpuntje in al die jaren was dat je gelijk bij start mijn kamer inpikte waardoor ik mijn spulletjes kon inpakken en naar de UNS 50 moest verhuizen! Gedurende onze promotiejaren hebben we heel wat kopjes koffie gedronken en spiekte ik altijd even door het raampje of je er was als ik op de UNS 40 was. Het bleef nooit bij eventjes bijpraten, maar vaak was ik dan weer een uur weg voordat ik de rest van mijn rondje op de UNS 40 afmaakte. Geregeld ging ons praatje over werk, al liggen onze promotie-onderwerpen echt mijlenver uit elkaar, en toch was dat nooit vervelend! Bedankt voor alle gezellige uren, en ik ben heel blij dat jij achter me staat tijdens 'dat ene uurtje'.

Beste Anke, jou ken ik natuurlijk nog veel langer, en al sinds de basisschool zijn we vriendinnen. Ook al zien we elkaar niet zo vaak, toch is het altijd vertrouwd en gezellig als we weer eens kunnen bijkletsen. Omdat jij ook gepromoveerd bent (en je hebt me nog ingehaald ook...) kon ik altijd mijn ei bij je kwijt, zowel privé als op gebied van wetenschap. We konden samen even roddelen over het lab en de analyses die gedaan moeten worden, en er kwam altijd een gelukkig appje als een manuscript geaccepteerd was. Ik waardeer je enthousiaste en eerlijke persoonlijkheid en ik ben ontzettend blij dat je mijn paranimf wil zijn!

Lieber Emanuel, jij kunt natuurlijk niet in dit rijtje ontbreken! Toen we elkaar leerden kennen op de TIFN kick-off bleek al uit de karaktertest die we moesten invullen dat jij en ik ongeveer elkaars tegenpolen waren. Dat kon twee kanten op gaan, maar dit heeft gelukkig buitengewoon goed uitgepakt! Onze eerste gezamenlijke testdagen waren een ramp en we stuntelden maar wat aan, maar aan het einde van de GOS studie waren we een geoliede machine en hadden we aan één woord genoeg. Het was altijd gezellig tijdens 
de testdagen, samen met de proefpersonen 'Sister Wives' kijken op TLC en alle zakjes met raar wit poeder afwegen (en de plakkerige keuken erna opruimen...). Ik wens je heel veel geluk voor de toekomst en ik weet zeker dat er ooit een professor Canfora gaat rondlopen.

Beste Dorien, wat een fantastische jaren hebben we gehad tijdens onze promotie. Jij draaide je eigen gigantische studie (waarvoor respect) waarbij ik af en toe binnen kwam hobbelen om de spierbiopten af te nemen. Tijdens onze promotietijd zijn we goede vriendinnen geworden en hebben we samen heel veel leuke (en minder leuke) dingen meegemaakt. Ik kon steeds bij je terecht als ik weer iets te bespreken had, en jij had ook altijd genoeg verhalen om de middagen te vullen. Gedurende de promotieperiode gingen we ook steeds vaker buiten werktijd met elkaar om en zo zagen we elkaar zeer regelmatig. De laatste tijd zien we elkaar natuurlijk wat minder vaak, mede door mijn tijd in Canada en jouw verblijf in Amerika, maar ik weet zeker dat we de draad gewoon weer oppakken als jij en Jasper terugkomen richting Nederland. Voor nu heel veel succes tijdens je avontuur in Amerika als postdoc!

Beste Imre, tijdens mijn echte promotiejaren hebben we elkaar niet zo vaak gezien en was ik voornamelijk 'biopteur' en 'de vriendin van'. Na mijn promotie zijn we echter huisgenootjes geworden en had je altijd een luisterend oor (en warm eten) als ik thuiskwam na een lange dag in Venlo. Ik weet niet hoe ik deze periode had doorstaan zonder jouw gekke verhalen, spontane etentjes en lekkere wijntjes op ons terras. Daarvoor veel dank!

Professor Blaak, beste Ellen. Ook al was je officieel geen promotor, toch was je zeer direct betrokken bij mijn onderzoek. Ik heb dan ook samen met Emanuel en Kaatje vele uren in jouw kantoor doorgebracht en we hebben mooi onderzoek kunnen verrichten. Heel veel dank voor je steun!

Beste MDL-collega's. Mede door jullie kijk ik met een grote glimlach terug op mijn promotietijd. Ik wil een paar mensen in het bijzonder noemen. Bouke en Chantal, jullie waren met jullie sterke verhalen een sensationeel onderdeel van de groep. Jullie waren altijd in voor een drankje en een avondje stappen en ik vind het super dat we in de toekomst collega's zullen worden! Ellen, je begon nog als studente van Bouke, maar al snel werd je een spontane en gezellige collega. Freddy, in mijn eerste jaren, en vooral tijdens de eerste katheterstudie, hebben we veel overleg gehad en kon ik steeds bij je terecht, dank daarvoor. Dan uiteraard de rest van de DDW-groep: Selwyn, Fedde, Ankie, Bram, Mark en Steven, wat hebben wij een ontzettend leuk congres in Washington gehad. Na mijn tweede presentatie, waarbij ik kneiter zenuwachtig was en jullie vooral heel erg brak in de zaal zaten, hebben we nog een mooie reis naar New York mogen maken. Uiteraard wil ik ook de rest van de onderzoekscollega's niet vergeten: Annemieke, Annick, Bas, 
Corinne, Daniël, Dirk, Elhaseen, Eveline, Fabiënne, Hao Ran, Kirsten, Lisa, Mariëlle, Marin, Marlou, Martine, Montserrat, Paul, Pauline, Roel, Roy, Samefko, Sander, Tim, Zlatan en Zsa Zsa. Samen hebben we een mooi fietsweekend in de Ardennen gehad, een gezellig skiweekend overleefd en organiseerden we frequent vrijdagmiddag-fiets-borrels. Alle MDL-opleidingsassistenten en MDL-artsen, bedankt voor jullie aandacht en input tijdens de research meetings, maar ook voor het plaatsen van onmogelijke colon-katheters bij niet volledig-voorbereide proefpersonen. Mietsie, het was altijd gezellig om even op het secretariaat langs te gaan en al kwam ik alleen voor het maken van een afspraak, er kwam altijd een glimlach en praatje bij!

Beste Heelkunde collega's. Ik had gedacht dat ik het als niet-snijdend persoon moeilijk zou krijgen tussen alle chirurgen, maar niets was minder waar! Speciaal dank aan mijn roomies: Inca, Kim en Bas. Na een aantal keer verhuizen kwam ik op de UNS 50 bij jullie terecht en zaten jullie opgescheept met de telefoontjes van mijn proefpersonen. Ik kon altijd mijn frustraties (aahhh Endnote) bij jullie kwijt en we hadden ook onze lol-momenten. Bas had het altijd maar zwaar tussen de drie dames! Heel veel dank aan alle laboranten, natuurlijk in het bijzonder Hans, ik geloof dat mijn SCFA-analyses je een paar jaar van je leven hebben gekost! Sander, als onderdeel van het TIFN team heb je zeker bijgedragen aan het wetenschappelijke niveau van dit boekje, bedankt! Mirjam, je begon als mijn studente en later ben je uitgegroeid tot collega en gewaardeerd co-auteur. Dank voor je inzet maar ook voor de gezelligheid tijdens de testdagen! Verder dank voor een mooie tijd aan (oud)collega's Anne-Claire, Annemarie, Audrey, Britt, Briete, Caroline, Charlotte, Claire, David, Dennis, Dirk, Edgar, Evelien, Fabio, Frank, Frans, Freek, Givan, Irene-Fleur, Jasper, Joyce-Manyi, Junfang, Kevin, Kiran, Kostan, Leontine, Liliane, Livia, Lori, Luc, Luuk, Mark, Martijn, Martine, Milou, Mo, Niek, Nina, Pieter, Rianne, Rob, Robbert-Jan, Rutger, Ruben, Steven, Thiemo, Tiara, Tim, Timme, Toine, Victor, Yee Lai en Zita.

Dan niet te vergeten de onderzoeksgroep bij de Humane Biologie en vooral Gabby. Ik kon altijd bij je terecht met moeilijke vragen over TIFN-procedures, al moest je daar weer urenlang achteraan. Jasper, je zorgde er niet alleen voor dat Dorien een stuk vrolijker was, maar je was ook altijd bereid om mee te denken over uitkomsten van analyses en je was nooit te beroerd om moeilijke SPSS-vragen te beantwoorden. Vele uren heb je besteed aan het afnemen van vetbiopten bij mijn proefpersonen, bedankt! Verder veel dank aan de laboranten, met speciale dank aan Jos, vele uren heb je gestoken in het analyseren van onze samples en met het organiseren van borrels en carnavalsfeesten. Antoine en Joan, ik ben een aantal keren naar boven gerend omdat de infuusplaatsing niet lukte, maar met jullie geduldige aanpak was er toch vaak binnen enkele minuten een goed lopend infuusje geplaatst, dank voor de snelle service! Gijs en Johan, als onderdeel van ons TIFN- 
team en copromotoren van Dorien en Emanuel waren jullie zeer nauw betrokken bij mijn onderzoek. Hartelijk dank voor alle feedback, maar ook voor de gezellige tijd onder andere tijdens onze TIFN-dagen.

Een paar jaar onderzoek doen bestaat natuurlijk niet alleen maar uit werken, en daarom wil ik ook nog een aantal mensen bedanken die niet direct betrokken zijn geweest bij mijn onderzoek. Beste Marc, je bent altijd oprecht geïnteresseerd geweest in mijn onderzoek en bij elke nieuwe ontwikkeling ging er weer een appje richting Leuven. Andersom krijg ik ook altijd updates over de voortgang van jouw eigen promotie en ben ik blij dat ook bij jou het einde in zicht is. Verder was je ook altijd in voor een etentje en stuurde je een filmpje uit de oude doos toen ik even een opsteker nodig had. Deze staat nog altijd in mijn inbox (beware...) voor als ik hem nodig heb! Yves, de jaren na ons bestuursjaar zijn we goed bevriend gebleven en was je altijd in voor een etentje of een drankje. Beste Esther, ook al ben je naar Utrecht verhuisd, ons contact is er niet door verwaterd. Ondanks al je gekke ideeën, waar ik niet altijd even hard in meega, ben je altijd zeer betrokken en kom je af en toe langs in het zonnige zuiden om een weekend bij te kletsen. Verder blijft het traditie om gelijk een foto van nieuw gekochte schoenen naar elkaar te sturen (en dat is ook heel belangrijk!). Uly en Inge, als niet-beta meisjes van onze fantastic four was het soms lastig om Anke en mij bij te houden als wij het over onze onderzoeken hadden. Respect dat jullie dat wel probeerden en in ieder geval oprecht geïnteresseerd waren! Dan alle dames van het Medisch Dames Dispuut Floreciente en vooral de lieve OLD-jes: Yrsa, Kelly en Jasmijn. Dank voor jullie steun en ik hoop op veel weekendjes weg de komende jaren!

Vervolgens nog een woord van dank aan alle collega's in Venlo. Dr. Hermans, beste Marc, bedankt voor je steun en de ruimte die ik kreeg om vier maanden tijdens de opleiding fulltime aan mijn onderzoek te besteden. Lieve assistent-collega's van de Interne Geneeskunde, nu het einde van mijn promotie in zicht is, zit mijn tijd in Venlo er ook alweer bijna op, alvast bedankt voor alle gezelligheid!

Papa, mama en Sanne. Jullie waren de afgelopen jaren, en vooral de laatste maanden, ontzettend betrokken bij mijn promotietraject. Ik begrijp dat het voor jullie soms nogal lastig was om te snappen waar ik me precies hele dagen mee bezig hield, maar ik kon altijd met mijn verhalen bij jullie terecht. De proefpersooninformatie die ik jullie toestuurde ter controle werd tot op de puntjes uitgeplozen om ervoor te zorgen dat alles duidelijk en grammaticaal correct was geschreven. Ook werd er altijd goed op me gelet, en als de koelkast dreigde leeg te raken en stonden jullie met bakjes eten voor me klaar. Vooral de laatste maanden had ik dat steuntje in de rug echt even nodig en daar ben ik jullie erg dankbaar voor. 
And last, but not least, lieve Stefan. Wij hebben elkaar tijdens ons beider promotietraject leren kennen en dat heeft er zeker ook toe geleid dat ik een fantastische onderzoekstijd heb gehad. Tijdens onze promotietijd gingen we samen van huis weg en fietsten we ook weer samen terug, liepen we af en toe bij elkaar naar binnen in kantoor, maar werkten we ook professioneel samen als ik spierbiopten bij jouw proefpersonen moest afnemen, of als ik even een handje hulp nodig had als Emanuel er niet was. Het feit dat ik zelfs een keer bij jou spierbiopten heb afgenomen (had ik genoeg materiaal eigenlijk?) geeft al aan dat er een groot vertrouwen is tussen ons. Vooral de afgelopen maanden ben je mijn steun en toeverlaat geweest, was je editor van al mijn stukken, en hielp je me er weer doorheen als de stress toesloeg. Ontzettend veel dank hiervoor. Ik ben trots op je dat je hebt durven kiezen voor je droom als postdoc in het buitenland en ik ben blij dat ik drie maanden samen met jou heb mogen meemaken hoe het is om als Nederlander in Canada te wonen. Ik kijk uit naar het moment dat je je carrière weer voortzet in Nederland en ik ben ervan overtuigd dat we een geweldige toekomst samen tegemoet gaan! 